\title{
New results from the low-frequency counterpart of the XMM large scale structure survey ${ }^{\star}$
}

\author{
C. Tasse ${ }^{1}$, A. S. Cohen ${ }^{2}$, H. J. A. Röttgering ${ }^{1}$, N. E. Kassim² ${ }^{2}$, M. Pierre ${ }^{3}$, R. Perley ${ }^{4}$, P. Best ${ }^{5}$, M. Birkinshaw ${ }^{6}$, \\ M. Bremer ${ }^{6}$, and H. Liang 6 \\ 1 Leiden Observatory, University of Leiden, PO Box 9513, 2300 RA Leiden, The Netherlands \\ e-mail: tasse@strw.leidenuniv.nl \\ 2 US Naval Research Laboratory, Remote Sensing Division, 4555 Overlook Avenue, SW, Code 7213, Washington, DC 20375, USA \\ 3 CEA/DSM/DAPNIA, Service d'Astrophysique, Saclay, 91191 Gif-sur-Yvette, France \\ ${ }^{4}$ National Radio Astronomy Observatory, Socorro, NM 87801, USA \\ 5 Institute for Astronomy, Royal Observatory Edinburgh, Blackford Hill, Edinburgh EH9 3HJ, UK \\ ${ }^{6}$ Department of Physics, University of Bristol, Tyndall Avenue, Bristol BS8 1TL, UK
}

Received 13 February 2006 / Accepted 16 May 2006

ABSTRACT

\begin{abstract}
The XMM Large Scale Structure survey (XMM-LSS) is an X-ray survey aimed at studying the large scale structure of the Universe. The XMM-LSS field is currently being followed up using observations across a wide range of wavelengths, and in this paper we present the observational results of a low frequency radio survey of the XMM-LSS field using the Very Large Array at 74 and $325 \mathrm{MHz}$. This survey will map out the locations of the extragalactic radio sources relative to the large scale structure as traced by the X-ray emission. This is of particular interest because radio galaxies and radio loud AGN show strong and complex interactions with their small and larger scale environment, and different classes of radio galaxies are suggested to lie at different places with respect to the large scale structure.

For the phase calibration of the radio data, we used standard self-calibration at $325 \mathrm{MHz}$ and field-base calibration at $74 \mathrm{MHz}$. Polyhedron-based imaging as well as mosaicing methods were used at both frequencies. At $74 \mathrm{MHz}$ we have a resolution of $30^{\prime \prime}$, a median $5 \sigma$ sensitivity of $\sim 162 \mathrm{mJy} /$ beam and we detect 666 sources over an area of 132 square degrees. At $325 \mathrm{MHz}$, we have a resolution of $6.7^{\prime \prime}$, a median $5 \sigma$ sensitivity of $4 \mathrm{mJy} /$ beam, and we detect 847 sources over an area of 15.3 square degrees. At $325 \mathrm{MHz}$ we have detected a region of diffuse radio emission which is a cluster halo or relic candidate.
\end{abstract}

Key words. techniques: interferometric - surveys - cosmology: observations - large-scale structure of Universe methods: data analysis - radio continuum: general

\section{Introduction}

Extragalactic radio sources such as radio loud quasars and radio galaxies, have been extensively studied to understand the physical processes relating active galactic nuclei (AGN), host galaxies, and environments. For powerful radio sources emission line and radio luminosities seem to be well correlated (McCarthy 1993), while at lower radio luminosities $\left(L_{1.4 \mathrm{GHz}} \lesssim\right.$ $10^{25} \mathrm{~W} \mathrm{~Hz}^{-1}$ ) radio loudness becomes decoupled from the AGN activity as probed by emission line luminosity (Zirbel \& Baum 1995; Best et al. 2005). It has been suggested that environment plays a major role in making a galaxy radio loud or radio quiet. In the local universe, FRI-type (Fanaroff \& Riley 1974) radio galaxies inhabit moderately rich cluster environments, while FRII-type radio sources tend to lie in either small groups or isolated fields (Yates et al. 1989; Hill \& Lilly 1991; Ledlow $\&$ Owen 1996). Furthermore a number of recent X-ray observations have shown a strong, FRI/FRII morphology-dependent coupling between steep spectrum radio emission and their surrounding intergalactic medium (IGM) (Fabian et al. 2003; Blanton et al. 2004; Fabian et al. 2005; Croston et al. 2005). The

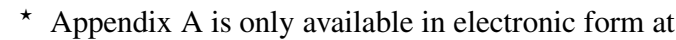
http://www.edpsciences.org question therefore arises as to what extent both AGN activity and environment properties are coupled with radio activity.

One way to statistically study the connections between various radio source populations and their environment is to compare X-rays emitted by the hot IGM plasma tracing Large Scale Structures (LSS), to low-frequency radio observations $(v<$ $1 \mathrm{GHz}$ ). The XMM-Large Scale Structure Survey (XMM-LSS) is an X-ray survey designed to investigate the nature, properties and content of the LSS in the Universe up to redshift $z \sim 1$ (Pierre et al. 2004). The XMM-LSS field is being observed by the XMM-Newton satellite and will cover 10 degree $^{2}$. It is predicted to detect $1500 \mathrm{X}$-ray quasars, and 100-200 galaxy clusters up to $z=1$, and $\sim 50$ within $1<z<2$ (Refregier et al. 2002). At present, $\sim 5$ degree $^{2}$ of the XMM-LSS field has been observed.

The XMM-LSS field will be mapped in five bands using the $1 \mathrm{deg}^{2}$ CCD camera MEGACAM as part of the Canada France Hawaï Telescope Legacy Survey $\left(\right.$ CFHTLS $\left.^{1}\right)$. The XMM-LSS field is also observed as part of the SWIRE (Lonsdale et al. 2003) survey in 9 bands between 3.6 and $24 \mu \mathrm{m}$. Spectroscopic follow-up (NTT, Magellan, VLT) of the $\sim 70$ galaxy cluster candidates found in the first 5 degree $^{2}$ of the survey is completed

\footnotetext{
${ }^{1}$ For more information on the CFHT Legacy Survey, see http://www.cfht.hawaii.edu/Science/CFHLS/
} 
Table 1. Observational parameters for the VLA radio survey of the XMM-LSS field.

\begin{tabular}{ccc}
\hline \hline Array configuration & $\mathrm{A}$ & $\mathrm{B}$ \\
\hline & 31 Jul. 03, & 15,16, \\
Obs. dates & 5 Aug. 03, & 17, 20 Jun. 02, \\
& 3 Sept. 03 & 16 Jul. 02 \\
Number of pointings & 4 & 4 \\
Int. time per pointing & $\sim 6 \mathrm{~h}$ & $\sim 8 \mathrm{~h}$ \\
Observing frequency & $73.8 / 325 \mathrm{MHz}$ & $73.8 / 325 \mathrm{MHz}$ \\
\hline & & \\
\hline & $73.8 \mathrm{MHz}$ & $325 \mathrm{MHz}$ \\
\hline Frequency & $1.56 \mathrm{MHz}$ & $6.25 \mathrm{MHz}$ \\
\hline Band width & 128 & $2 \times 16$ \\
$N_{\text {channel }}$ & $12.20 \mathrm{kHz}$ & $195.31 \mathrm{kHz}$ \\
Channel width & $3 \mathrm{C} 405$ & $3 \mathrm{C} 48$ \\
Band Pass calibrator & $3 \mathrm{C} 405$ & $3 \mathrm{C} 48$ \\
Flux calibrator & $30^{\prime \prime}$ & $6.7^{\prime \prime}$ \\
Resolution & 132 & 15.3 \\
Area (degree ${ }^{2}$ ) & $162 \mathrm{mJy} / \mathrm{Beam}$ & $4.0 \mathrm{mJy} / \mathrm{Beam}$ \\
Sensitivity (at $5 \sigma)$
\end{tabular}

(Pierre et al. 2006; Pacaud et al. 2006, in preparation). Standard spectroscopic follow-up of the SWIRE and XMM sources is underway at the 2-degree field spectrograph (2dF). Combining these data will provide an unprecedented view of the LSS of the universe (see Pierre et al. 2004, for a general lay-out of the associated surveys).

Using the Very Large Array (VLA) radio telescope, we have begun a low frequency radio survey at 325 and $74 \mathrm{MHz}$ of the XMM-LSS field in order to address the following questions: (1) where are different classes of radio sources located with respect to the distribution of mass on cosmological scales as traced by the X-ray emission? (2) Can the radio loud/quiet aspect of optical and X-ray quasars be understood in terms of environmental effects? (3) How does the hot IGM influence the physical properties of the radio sources such as linear size and radio power?

In this paper we describe the observations we have conducted using the VLA in July 2003 in the A-configuration (most extended) and in June 2002 in the B-configuration. This combination provides resolutions of $6.7^{\prime \prime}$ and $30^{\prime \prime}$ at 325 and $74 \mathrm{MHz}$, respectively. Following the observational strategy described in Cohen et al. (2003), but using a mosaic of four pointings, at $325 \mathrm{MHz}$ we cover a $\sim 15$ degree $^{2}$ area with a resolution of $6.7^{\prime \prime}$ and a mean flux density limit per beam $(5 \sigma)$ of $\sim 4 \mathrm{mJy} /$ Beam. At $74 \mathrm{MHz}$, we cover a 132 degree $^{2}$ area with a resolution of $30^{\prime \prime}$ and a flux density limit per beam of $\sim 160 \mathrm{mJy} /$ Beam. A summary of these results appear in Table 1.

Figure 1 shows the sensitivity and resolution of the XMM-LSS low-frequency counterpart, at 74 and $325 \mathrm{MHz}$, compared with others radio surveys. At $325 \mathrm{MHz}$, the lowfrequency survey of the XMM-LSS field is deeper than the WENSS survey (Rengelink et al. 1997) by a factor of $\sim 3$, and in resolution by a factor of $\sim 10$. At $74 \mathrm{MHz}$, we exceed the VLSS (Cohen et al. 2006) by a factor of $\sim 3$ in both sensitivity and resolution. The NVSS survey (Condon et al. 1998) covers the whole XMM-LSS field, and most of our $74 \mathrm{MHz}$ sources will have a counterpart at $1.4 \mathrm{GHz}$, whereas our $325 \mathrm{MHz}$ data is deep enough so that many sources will not have detected counterparts in the NVSS. Compared with the VLA-VIRMOS deep field at 1.4 GHz (Bondi et al. 2003), reaching a brightness temperature limit at $5 \sigma$ of $\sim 90 \mu \mathrm{Jy} /$ Beam, probing nearby starbursts over $\sim 1$ degree $^{2}$ area, we are probing powerful AGN over a larger area.
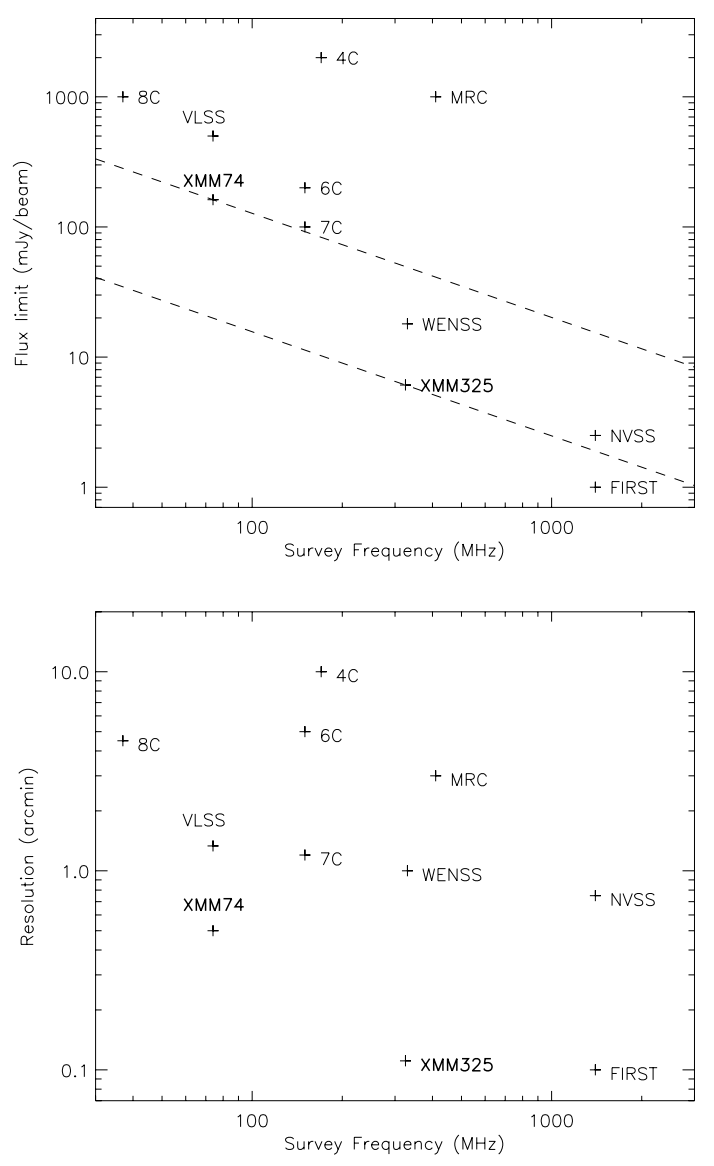

Fig. 1. Sensitivity and resolution of our XMM-LSS radio surveys, in comparison with that of other surveys. The dashed lines represent fiducial sources with a spectral index $\alpha=-0.8$.

This paper is organized as follows. In Sect. 2 we describe the observations, and the data reduction. In Sect. 3 we describe the sources extraction and we study the reliability/completeness aspects of the source lists. Section 4 presents the final results and we conclude in Sect. 5 by discussing the survey, and future plans.

\section{Observations}

The XMM-LSS field ${ }^{2}$ is centered at $\alpha(\mathrm{J} 2000)=2^{\mathrm{h}} 24^{\mathrm{m}} 00.27^{\mathrm{s}}$, $\delta(\mathrm{J} 2000)=-4^{\circ} 09^{\prime} 47.6^{\prime \prime}$ (Pierre et al. 2004). This location was chosen because of its high Galactic latitude and low extinction. The declination near the equator also gives the advantage of being visible from many astronomical observatories.

\subsection{Observational strategy}

The first radio observations at 74 and $325 \mathrm{MHz}$ of the XMM-LSS field were carried out with the VLA (Cohen et al. 2003 ) in an $8 \mathrm{~h}$ run. It covered 5.6 degree $^{2}$ at $325 \mathrm{MHz}$ with a resolution of $6.3^{\prime \prime}$, and reached a flux density limit per beam of $4 \mathrm{mJy}^{\text {beam }}{ }^{-1}(5 \sigma)$, leading to the detection of 256 sources. At $74 \mathrm{MHz}$ the primary beam covered a 110 degree $^{2}$ area with a resolution of $30^{\prime \prime}$, and a flux density limit per beam of $275 \mathrm{mJy}_{\text {beam }}^{-1}(5 \sigma)$, leading to the detection of 211 sources.

\footnotetext{
${ }^{2}$ for more information on the present status of the XMM-LSS survey see http://vela.astro.ulg.ac.be/themes/spatial/xmm/LSS/ index_e.html.
} 
We carried out a $24 \mathrm{~h}$ observation of the XMM-LSS field simultaneously at 74 and $325 \mathrm{MHz}$ in the A-configuration. This observation was spread over July, August and September 2003. At $325 \mathrm{MHz}$, we have added data from a $35 \mathrm{~h}$ observing run in the B-configuration, observed in June and July 2002. The observational parameters are listed in Table 1. The A-configuration gives us the needed high resolution to determine morphologies of the radio sources, and the $\mathrm{B}$ configuration is used for the determination of reliable flux densities and provides sensitivity to large angular scale emission needed, for example, to detect giant radio halos. At $74 \mathrm{MHz}$ the primary beam is large enough to cover the whole XMM-LSS field in a single pointing, and at $325 \mathrm{MHz}$ the pointing grid has been set so that we cover $\sim 90 \%$ of the XMM-LSS field with four pointings.

\subsection{Data reduction}

For the data reduction, we used the Astronomical Image Processing System (AIPS).

\subsubsection{The $325 \mathrm{MHz}$ data}

After a first round of flagging data affected by Radio Frequency Interference (RFI), we calibrated the relative response within each channel (i.e. bandpass), and performed gain and amplitude calibration. On the calibrated data, RFI was removed manually using the AIPS's routines TVFLG and SPFLG. In each of the four pointings $\sim 15 \%$ of the data have been flagged. We combined the $\mathrm{A}$ and $\mathrm{B}$ configuration data in the $u-v$ plane using the AIPS routine DBCON.

The phase calibrator we used (3C48) being far from the field, the initial phase calibration is very poor. Assuming a median spectral index $\alpha_{325}^{1400}=-0.8$, we generate a model of the sky at $325 \mathrm{MHz}$ from the NVSS database at $1.4 \mathrm{GHz}$ (Condon et al. 1998). This model consists of a list of clean components located at the position of the sources from the NVSS survey. Fourier transforming the modeled image allows for a phase-only calibration of each antenna using a solution interval of one minute, providing better results than the traditional calibrator-based phase calibration.

Because of the very large primary beam and the noncoplanar geometry of the VLA array, imaging would normally require the use of a 3D Fourier transform. However, this is currently too computationally expensive to be practical. The commonly used solution is to compute a pseudo-three-dimensional Fourier transform (Perley 1999), in which the field of view is divided into much smaller fields (facets). The 3D Fourier transform can then be approximated by using a two dimensional one. For the $325 \mathrm{MHz}$ data, we used 286 facets, each $512 \times 512$ pixels, sampled at $1.5^{\prime \prime}$ pixel $^{-1}$, with an overlap of two pixels between the facets. After the $u-v$ data have been imaged into individual facet and deconvolved, the facets are combined into a single image. After a few iterations of phase-only self-calibration, we combined the four pointings (each 2.5 degree in diameter) as described by Condon et al. (1998) in a $15.3 \mathrm{deg}^{2}$ single map.

The resulting noise is quite inhomogeneous across the field, ranging between $\sim 0.5$ and $\sim 2.5 \mathrm{mJy}^{\text {beam }}{ }^{-1}$. The noise is higher close to the bright sources, where sensitivity is dynamic-range limited. In each of the four pointings, the size of the synthesized beam was $\sim 6.7^{\prime \prime} \times 6.3^{\prime \prime}$ and we have set the restoring beam to be a circular Gaussian with a 6.7" FWHM.

\subsubsection{The $74 \mathrm{MHz}$ data}

For the $74 \mathrm{MHz}$ data, we used Cygnus-A (3C 405) as bandpass and flux density calibrator. Due to its large angular size, the calibrator is resolved by our observation. To calibrate the data, we used a standard model available from previous observations.

The problems of RFI and the non-coplanar geometry are solved in a similar way as with the $325 \mathrm{MHz}$ data. However, at $74 \mathrm{MHz}$ the ionosphere poses an additional challenge. Electrons in the ionosphere produce distortion of the wavefront and the resulting phase shifts $\Delta \phi$ increases linearly with the wavelength (Kassim et al. 1993). Moreover at higher frequencies the primary beam is relatively smaller in size so that angle-variant phase shifts across the field-of-view can be ignored and standard self-calibration can be utilized to derive one time variable phase correction per antenna. Below $150 \mathrm{MHz}$ positional-dependent phase variations become significant, and simple angle-invariant self-calibration breaks down. Therefore at $74 \mathrm{MHz}$ we have used the technique of "field-based calibration" first developed for the VLSS survey in which the phase calibration is position-dependent across the field-of-view (Cotton et al. 2004). This technique was also used and discussed into much details in Cohen et al. (2003).

Combining the A and B-configuration data in the UV-plane didn't lead to much improvement, probably because of complications of the ionospheric calibration routine. We therefore only considered the higher resolution A-configuration data. For each pointing, after the uv plane has been imaged in the 184 facets, we used a circular Gaussian restoring beam of $30^{\prime \prime}$ FWHM. The four pointings have then been combined into a single map. As with the $325 \mathrm{MHz}$ data, the noise is inhomogeneous across the field and as low as $\sim 20 \mathrm{mJy} /$ Beam and as high as $\sim 55 \mathrm{mJy} /$ Beam near bright sources.

\section{Source list}

\subsection{Detection}

As a first step in our source finding algorithm, we have normalized the image by a noise map, produced using AIPS's task RMSD. The noise is calculated within windows, fitting a Gaussian to the histogram of the pixel values. The data above and below the $3 \sigma$ domain are rejected, and after 30 iterations, a reliable estimation of the noise is obtained. The size of the window is critical since if it is too small, the noise evaluation will be overestimated by the presence of a strong signal, while if it is too big, it will not take into account the smaller scale variation in the noise pattern.

We set the window to be $80 \times 80$ pixels, corresponding to $10^{\prime} \times 10^{\prime}$ and $2^{\prime} \times 2^{\prime}$, at 74 and $325 \mathrm{MHz}$, respectively, and in order to save computing time, the rms is evaluated every $3 \times 3$ pixel, which is of the order of the correlation length. This introduces pixel-to-pixel uncorrelated noise, and in order to avoid for discontinuous variation in the noise level, we convolve this noise map with a circular Gaussian with diameters of $100^{\prime \prime}$ and $20^{\prime \prime}$ at 74 and $325 \mathrm{MHz}$ respectively which slightly smooths the noise image. Figure 2 shows the area mapped as a function of sensitivity for each frequency.

Dividing the original map by the noise map we get an image containing uniform noise, where we can apply the AIPS's source extraction algorithm "Search And Destroy" (SAD). SAD is given an input cut of 5 in the noise-normalized map $(\$ 5 \sigma$ in the original map) applied on both peak and integrated flux density, above which each pixel group is considered as a 


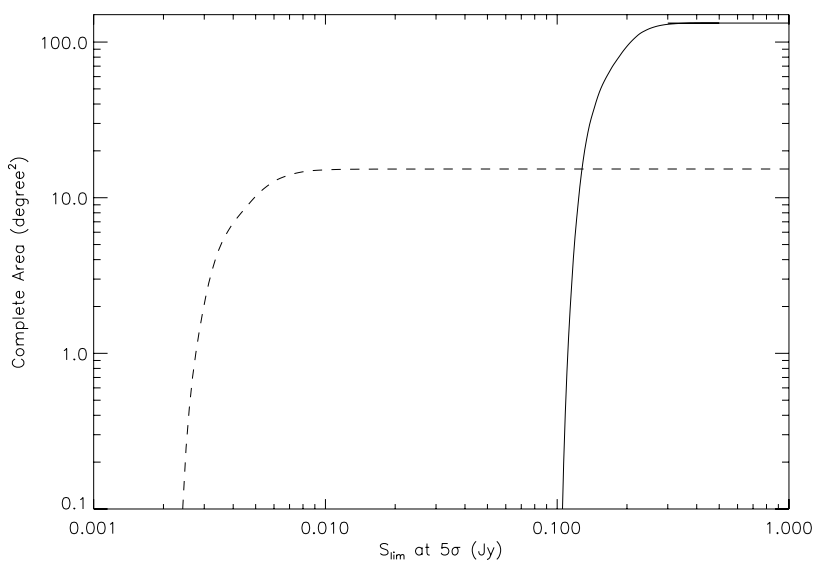

Fig. 2. Area as a function of limiting flux density per beam at $5 \sigma$. The full line corresponds to the $74 \mathrm{MHz}$ survey whereas the dashed one corresponds to the $325 \mathrm{MHz}$ survey.

potential source (island). SAD fits one or more Gaussian components to these islands, thereby producing an initial source list. Assuming the noise distribution to be Gaussian, the input cut of $5 \sigma$ leads to a total number of false detection over the surveyed areas of $\lesssim 5 \times 10^{-2}$ at 325 and $74 \mathrm{MHz}$. A visual inspection of the residual map does reveal some false detections, due to the non-Gaussian, correlated nature of the noise in the proximity of bright sources. We have deleted these false detections from the list while comparing the source list positions with NVSS as described below.

\subsection{Noise dependent errors}

Finally, the absolute flux densities are obtained by multiplying the measured noise-normalized flux density by the local noise. Following Condon (1997) we calculate the true uncertainties, from the signal-to-noise ratio of the Gaussian fit $\rho$, as expressed by:

$\rho^{2}=\frac{\theta_{\mathrm{M}} \theta_{\mathrm{m}}}{4 \theta_{\mathrm{N}}^{2}}\left[1+\left(\frac{\theta_{\mathrm{N}}}{\theta_{\mathrm{M}}}\right)^{2}\right]^{\alpha_{\mathrm{M}}}\left[1+\left(\frac{\theta_{\mathrm{N}}}{\theta_{\mathrm{m}}}\right)^{2}\right]^{\alpha_{\mathrm{m}}} \frac{S_{\mathrm{P}}^{2}}{\sigma_{\text {map }}^{2}}$

where $\theta_{\mathrm{M}}$ and $\theta_{\mathrm{m}}$ are fitted $F W H M \mathrm{~s}$ of the major and minor axes, $\theta_{\mathrm{N}}$ is the $F W H M$ of the Gaussian correlation length of the image noise, corresponding to the $F W H M$ of the synthesized beam, $S_{\mathrm{P}}$ is the peak flux density, and $\sigma_{\text {map }}^{2}$ is the local noise variance. $\left\{\alpha_{\mathrm{M}}, \alpha_{\mathrm{m}}\right\}$ have values determined empirically using Monte-Carlo simulations (Condon 1997, see Table 2). We have then calculated the errors of the fitted parameters as follows:

$$
\begin{gathered}
\frac{\sigma^{2}\left(S_{\mathrm{P}}\right)}{S_{\mathrm{P}}^{2}}=8 \ln 2 \frac{\sigma^{2}\left(x_{0}\right)}{\theta_{\mathrm{M}}^{2}}=8 \ln 2 \frac{\sigma^{2}\left(y_{0}\right)}{\theta_{\mathrm{m}}^{2}}=\frac{\sigma^{2}\left(\theta_{\mathrm{M}}\right)}{\theta_{\mathrm{M}}^{2}} \\
=\frac{\sigma^{2}\left(\theta_{\mathrm{m}}\right)}{\theta_{\mathrm{m}}^{2}}=\frac{\sigma^{2}(\phi)}{2}\left(\frac{\theta_{\mathrm{M}}^{2}-\theta_{\mathrm{m}}^{2}}{\theta_{\mathrm{M}} \theta_{\mathrm{m}}}\right)^{2} \approx \frac{2}{\rho^{2}} \\
\frac{\sigma^{2}\left(S_{\mathrm{T}}\right)}{S_{\mathrm{T}}^{2}} \approx \frac{\sigma^{2}\left(S_{\mathrm{P}}\right)}{S_{\mathrm{P}}^{2}}+\left(\frac{\theta_{\mathrm{N}}^{2}}{\theta_{\mathrm{M}} \theta_{\mathrm{m}}}\right)\left(\frac{\sigma^{2}\left(\theta_{\mathrm{M}}\right)}{\theta_{\mathrm{M}}^{2}}+\frac{\sigma^{2}\left(\theta_{\mathrm{m}}\right)}{\theta_{\mathrm{m}}^{2}}\right) .
\end{gathered}
$$

Here $S_{\mathrm{T}}$ is the total flux density, $\phi$ is the position angle of the major axis. $\sigma\left(x_{0}\right)$ and $\sigma\left(y_{0}\right)$ are related to the uncertainties in
Table 2. Values of $\left\{\alpha_{\mathrm{M}}, \alpha_{\mathrm{m}}\right\}$ used for the calculation of error bars on individual parameters (Condon 1997).

\begin{tabular}{ccc}
\hline \hline Parameter & $\alpha_{\mathrm{M}}$ & $\alpha_{\mathrm{m}}$ \\
\hline$S_{\mathrm{p}}$ & $3 / 2$ & $3 / 2$ \\
$\theta_{\mathrm{M}}, x_{0}$ & $5 / 2$ & $1 / 2$ \\
$\theta_{\mathrm{m}}, y_{0}, \phi$ & $1 / 2$ & $5 / 2$ \\
\hline
\end{tabular}

right ascension and declination (respectively $\sigma_{\alpha, \text { fit }}$ and $\sigma_{\delta, \text { fit }}$ ) by the relations given by Condon et al. (1998):

$\sigma_{\alpha, \text { fit }}^{2}=\sigma^{2}\left(x_{0}\right) \sin ^{2}(\phi)+\sigma^{2}\left(y_{0}\right) \cos ^{2}(\phi)$
$\sigma_{\delta, \text { fit }}^{2}=\sigma^{2}\left(x_{0}\right) \cos ^{2}(\phi)+\sigma^{2}\left(y_{0}\right) \sin ^{2}(\phi)$.

\subsection{Calibration errors}

- Position errors: the imperfect phase calibration adds positional uncertainties. We can quantify these by comparing our astrometry measurements to a much more accurate source positioning catalog. Figure 3 shows all the position differences between the NVSS survey and our source samples at both 74 and $325 \mathrm{MHz}$ on both right ascension and declination. At $325 \mathrm{MHz}$, the mean value of the position differences do not show any significant offset, while at $74 \mathrm{MHz}$ we measure an average offset of $2.25^{\prime \prime}$ and $-0.4^{\prime \prime}$ on respectively right ascension and declination. We have removed these offsets in the final source list. The scatter around the NVSS positions is given by:

$\sigma_{\alpha}^{2}=\epsilon_{\alpha, \mathrm{calib}}^{2}+\epsilon_{\alpha, \mathrm{NVSS}}^{2}+\sigma_{\alpha, \mathrm{fit}}^{2}$

$\sigma_{\delta}^{2}=\epsilon_{\delta \text {,calib }}^{2}+\epsilon_{\delta, \mathrm{NVSS}}^{2}+\sigma_{\delta \text {,fit }}^{2}$

where $\epsilon_{\alpha, \text { calib }}$ and $\epsilon_{\delta \text {,calib }}$ are the calibration errors due to the ionosphere, $\epsilon_{\alpha, \mathrm{NVSS}}$ and $\epsilon_{\delta, \mathrm{NVSS}}$ are the calibration errors of the NVSS sources, and $\sigma_{\alpha \text {,fit }}$ and $\sigma_{\delta \text {,fit }}$ are the Gaussian fitting errors (Eqs. (1)-(5)).

At $325 \mathrm{MHz}$, the scatter in the distribution of the positional differences between the $325 \mathrm{MHz}$ single source population and their associated NVSS counterpart contains the fitting errors of both ours and NVSS databases, as well as calibration errors. Therefore, selecting only the sources with higher signal-to-noise ratio lower the noise-dependent error contributions. Selecting the $325 \mathrm{MHz}$ sources with associated NVSS counterparts having error bar lower than $0.6^{\prime \prime}$ gives a subsample of 41 sources with associated Gaussian fitting error contributions on the level of $0.05^{\prime \prime}$. For this high signal-to-noise ratio subsample, we find scatters of $\sigma_{\alpha}=1.15^{\prime \prime}$ and $\sigma_{\delta}=1.83^{\prime \prime}$. Compared with the NVSS noise-independent uncertainties of $\epsilon_{\alpha, \mathrm{NVSS}}=0.45^{\prime \prime}$ and $\epsilon_{\delta, \mathrm{NVSS}}=0.56^{\prime \prime}$, our values are much higher. We can explain this difference by the fact that we have used an NVSS-based model to phase calibrate the data which included low signal-to-noise NVSS sources, where noise-dependent uncertainties dominate noise-independent ones. Therefore, we consider the quadratic differences between our measurement of the position uncertainties of respectively $1.15^{\prime \prime}$ and $1.83^{\prime \prime}$ and the NVSS calibration errors to be a good estimate of our calibration errors. This leads to $\epsilon_{\alpha, \text { calib }}=1.06^{\prime \prime}$ and $\epsilon_{\delta \text {, calib }}=1.75^{\prime \prime}$

At $74 \mathrm{MHz}$, we get scatter values of $\sigma_{\alpha}=6.8^{\prime \prime}$ and $\sigma_{\delta}=3.7^{\prime \prime}$ for the whole population, which contains uncertainties coming from both Gaussian fitting errors and calibration errors, as for the $325 \mathrm{MHz}$ data. Although the NVSS resolution is lower than ours, we assume the position errors from NVSS to be negligible, as the signal-to-noise ratio of NVSS is on 

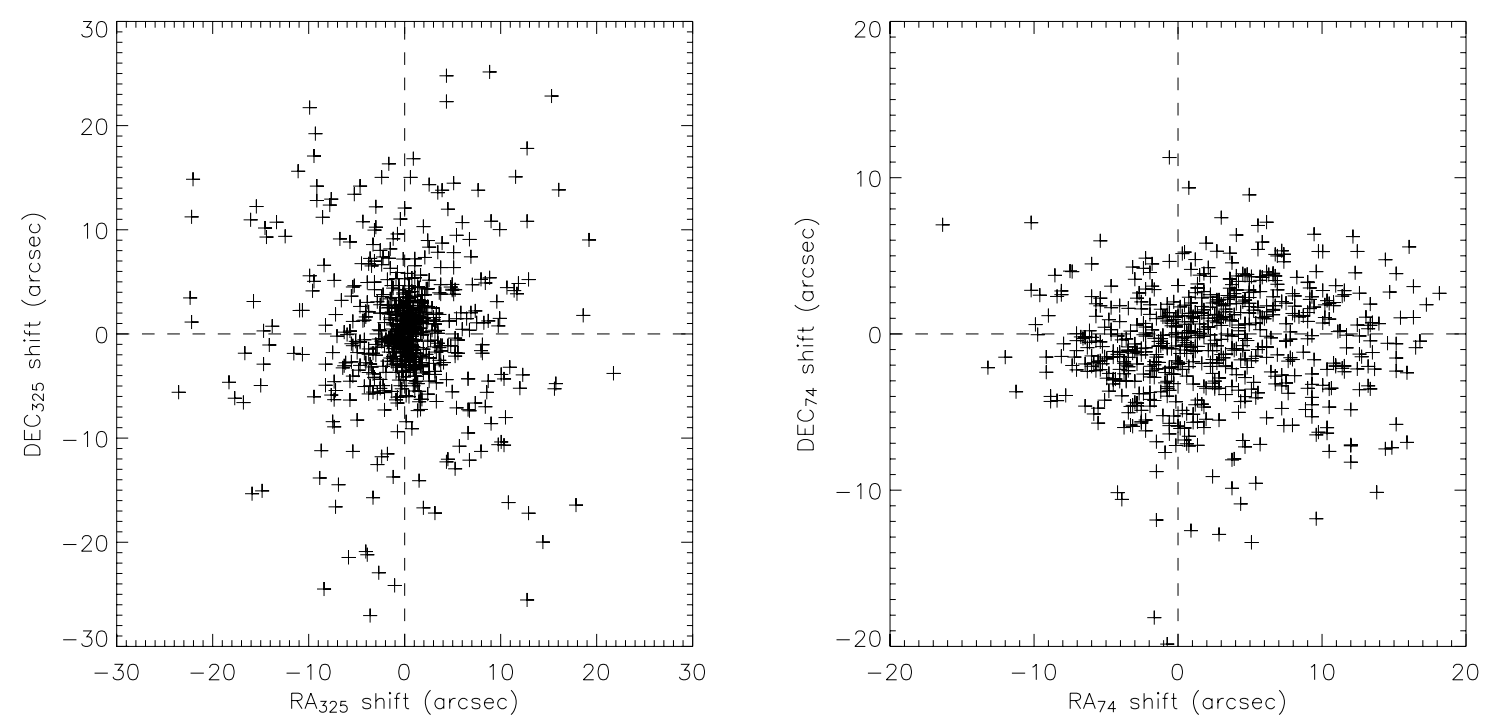

Fig. 3. Positional differences in right ascension and declination between our source sample and NVSS at 325 (on the left) and $74 \mathrm{MHz}$ (on the right). The $74 \mathrm{MHz}$ source sample corresponds to a much brighter source population in NVSS than the $325 \mathrm{MHz}$ source sample counterpart, so that the scatter in positional differences on the right reflects the $74 \mathrm{MHz}$ dataset calibration errors. At $325 \mathrm{MHz}$, the scatter is dominated by the NVSS uncertainties.

average $\sim 10$ times greater (see Fig. 1). In order to quantify the calibration errors, we select sources with $\sigma_{\alpha, \text { fit }}$ and $\sigma_{\delta \text {,fit }}$ lower than $0.5^{\prime \prime}$, which makes a subsample of 43 sources detected at high signal-to-noise ratio. We find standard deviations in right ascension and declination of respectively $\sigma_{\alpha}=3.43^{\prime \prime}$ and $\sigma_{\delta}=2.14^{\prime \prime}$, which, subtracting the NVSS calibration error contribution, leads to $\epsilon_{\alpha, \text { calib }}=3.37^{\prime \prime}$ and $\epsilon_{\delta \text {, calib }}=2.00^{\prime \prime}$. We have quadratically added these errors to the Gaussian fitted ones.

- Flux density errors: for the flux density calibration, we have used $3 \mathrm{C} 48$, and $0137+331$, at 74 and $325 \mathrm{MHz}$ respectively. We have assumed the flux density of these sources to be reliable at the level of $5 \%$ (see Cohen et al. 2003). We therefore have an uncertainty at the level of $5 \%$ on the overall flux density scale. We have quadratically added that uncertainty value to the noise-based Gaussian fitting error, given for the peak and integrated flux densities.

- Source size errors: as discussed in detail by Cohen et al. (2003), at $74 \mathrm{MHz}$ incompletely corrected ionospheric effects, which are similar to "seeing" effects in the optical domain, are hard to quantify, as we would need to know the actual source sizes. In order to evaluate the effects of the seeing we define the fitted size of resolved source of diameter $\theta_{\text {source }}^{2}$ to be:

$\theta_{\text {fit }}^{2}=\theta_{\text {source }}^{2}+\theta_{\text {beam }}^{2}+\theta_{\text {seeing }}^{2}$

Here $\theta_{\text {beam }}$ is the beam size, and $\theta_{\text {seeing }}$ corresponds to the size of a point source, deconvolved from the beam and imaged with that level of seeing. Figure 4 shows the scatter of the extendedness of the whole source population at $74 \mathrm{MHz}$ as estimated by $S_{\mathrm{t}} / S_{\mathrm{p}}$.

Although we do not have any information on the actual source sizes of the individual sources, assuming the bulk of the source population is unresolved, we can directly get an upper limit on the actual value of the seeing. The median value $\operatorname{med}\left(S_{\mathrm{t}} / S_{\mathrm{p}}\right)=1.48$ gives an upper limit on the seeing of 20.7", and in order to be conservative, we have considered its lower limit to be $0^{\prime \prime}$.

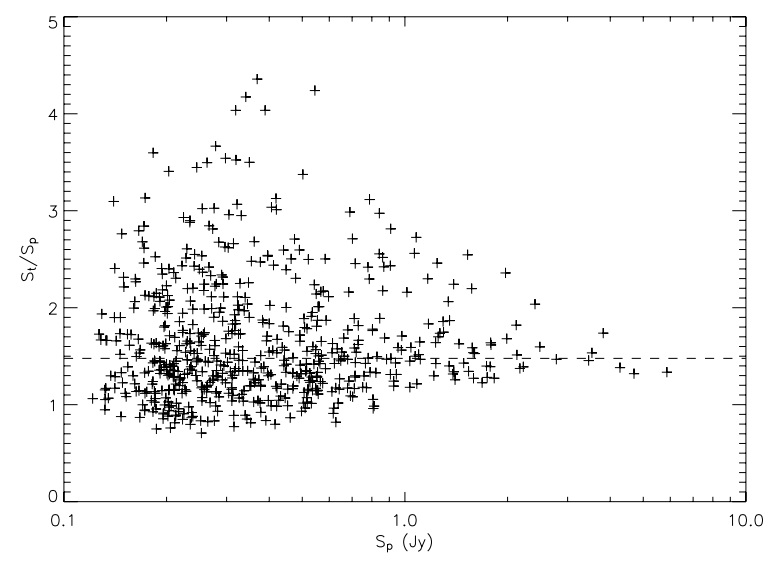

Fig. 4. At $74 \mathrm{MHz}$ : the ratio of the integrated to the peak flux density of each source vs. the peak flux density. Even if we cannot disentangle the discrepancy between the effects of seeing and physical size, for the seeing effect estimation we take the median value as a good upper limit (dashed line), assuming that the sources with that size are actually unresolved.

\subsection{Completeness}

In order to quantify the source detection efficiency, we have computed a Monte-Carlo simulation, generating 1000 sources with peak flux densities between 4 and $12 \sigma$ in a $2800 \times$ 3100 pixel image, cut from the residual map. Figure 5 shows the number of undetected sources as a function of the signal-tonoise ratio. A function of the following form, gives a fit to the Monte-Carlo outputs:

$f_{\mathrm{m}}\left(S_{\mathrm{p}} / \sigma_{l}\right)=1.13\left(\frac{S_{\mathrm{p}}}{\sigma_{1}}-3.67\right)^{-2.68}$

where $S_{\mathrm{p}}$ is the peak flux density and $\sigma_{1}$ is the local noise value. We can see that $\sim 95 \%$ of the sources are detected above $7 \sigma$, and this value could be a reasonable estimation of the completeness level. Though assuming the missed fraction to be known, we can correct the source counts estimation by compensating down to the $5 \sigma$ level the SAD detection inefficiency. We define 


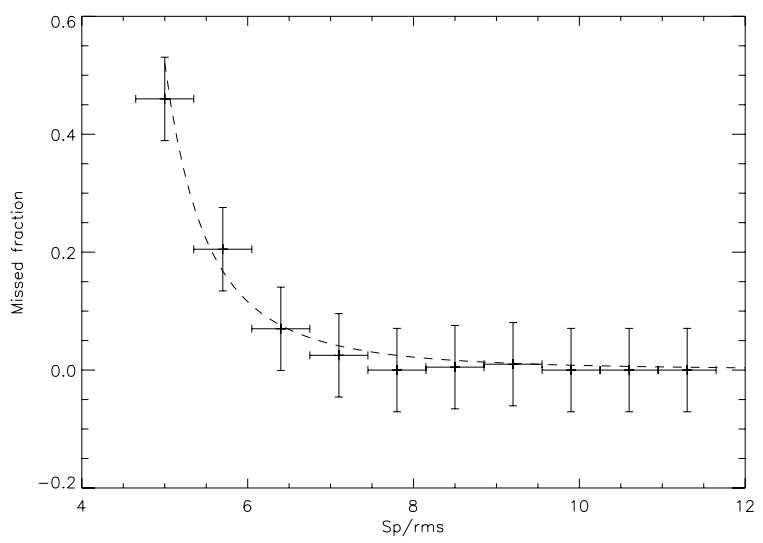

Fig. 5. The fraction of the missed sources as a function of SNR, from Monte-Carlo simulation, and fitted curve (dashed line).

an effective area element as the integration of a surface element weighted by the detection efficiency $\left(1-f_{\mathrm{m}}\left(S_{\mathrm{p}} / \sigma_{1}\right)\right)$. The total effective area at each flux density level $S$ is the integration of that quantity over the domain where the local noise $\sigma_{1}$ is as $\sigma_{1}<S / 5$ :

$A_{\mathrm{eff}}(S)=\int_{\sigma_{1}<S / 5}\left[1-f_{\mathrm{m}}\left(S_{\mathrm{p}} / \sigma_{1}\right)\right] \mathrm{d} A$.

The source count estimator $N\left(>S_{0}\right)$ is then corrected as follows:

$N_{\mathrm{c}}\left(>S_{0}\right)=\int_{S_{0}}^{+\infty} \frac{n(S) \mathrm{d} S}{A_{\mathrm{eff}}(S)}$.

We have computed the uncertainties from the source counts, assuming Poisson statistics:

$\sigma^{2}\left(N>S_{0}\right)=\sum_{i} \frac{1}{A_{i}^{2}} N_{i}$.

Here $N_{i}$ is the number of sources per bin, and $A_{i}$ is the area used in the source count calculation.

\subsection{Extended flux density estimation}

The source extraction method using Gaussian fitting algorithm can lead to an underestimation of the integrated flux densities, when significant part of the emission are extended. In order to quantify this effect, we derive another estimation of the integrated flux density inside a $60^{\prime \prime}$ diameter aperture, centered at the position of each Gaussian component. Then we have $S_{\text {int }}=\Sigma \times 4 \ln 2 /(\pi . F W H M)$, where $S_{\text {int }}$ is the integrated flux density, $\Sigma$ is the sum of the pixels inside the aperture, and $F W H M$ is the Full Width at Half Maximum in pixels. Figure 6 shows the cumulative probability distribution of the noise-normalized difference between the two flux density estimates for subsamples of unresolved point-like sources, and resolved, or multiple sources. Using a Kolmogorov-Smirnoff test, we compare both distributions with a purely Gaussian distribution. We derive probability values for the distributions to be Gaussian of $P_{\mathrm{KS}} \sim 0.7$ for point like sources and $P_{\mathrm{KS}} \ll 10^{-4}$ for extended sources, indicating that the flux density estimates by the two methods are in agreement for point-like sources and obviously disagree for resolved sources. The median value of the ratio between the flux densities derived from the two methods for extended sources gives the average bias to be $\sim 7 \%$. Therefore, at $325 \mathrm{MHz}$, since the

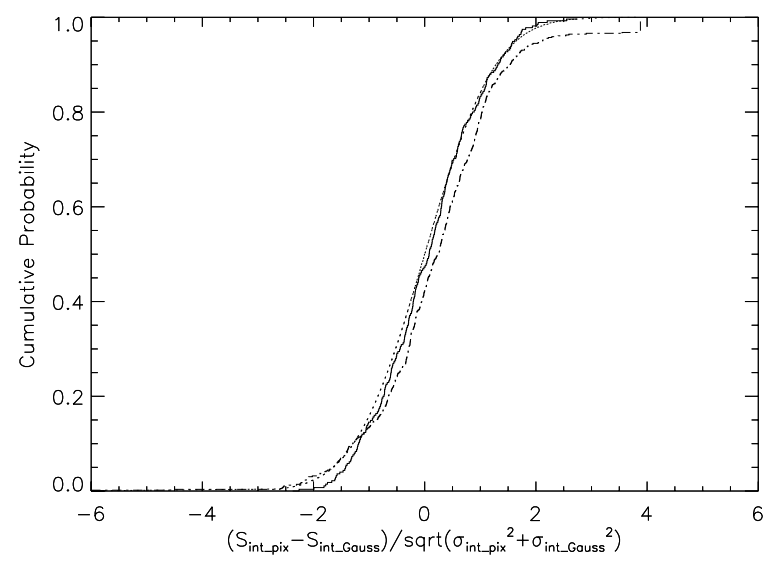

Fig. 6. The cumulative probability distribution of the noise-normalized difference between the two flux density estimates derived by the Gaussian fitting method ("int_Gauss" label ) and the pixel-based method ("int_pix" label). The solid line represents a subsample of single, unresolved sources, whereas the dash-dotted line represents the extended sources. The purely Gaussian distribution is over-plotted the dotted line.

error bars generated by the pixel based method are much higher, when the significance of the difference between the two estimates is above $2 \sigma$, we derive the integrated flux density following the pixel-based method, rather than summing over the Gaussian component individual flux densities. At $74 \mathrm{MHz}$, the resolution being much larger, most sources are unresolved and the Gaussian fitting based integrated flux density estimation is reliable.

\section{Results}

\section{1. $325 \mathrm{MHz}$ results}

At $325 \mathrm{MHz}$ using the extraction method described above, we extract 877 sources from the 15.3 degree $^{2}$ combined map. This sample contains a significant number of obviously false detections and by visual inspection, we rejected 30 that were close to the brightest sources where the noise is non-Gaussian.

We have defined as multiple sources those separated by less that $60^{\prime \prime}$, and assuming Poissonian statistics this makes the probability of two independent sources to be classified as multiple lower than $1 \%$. We finally arrive at a list of 847 sources in which 621 are single ("S"), and 226 are multiple ${ }^{3}$ ("M"). We have defined as unresolved sources the ones distinguishable from the beam size at the $2 \sigma$ level, and on the 621 single component sources, 484 where unresolved. The final source list appears in Table A.1. Images of the multiple component sources larger than twice the beam size are shown in Fig. A.1.

Since the Cohen et al. (2003) radio sources are all detected in our deeper and wider survey, at the same frequency, we can directly compare their flux densities. We found that Cohen et al. (2003) flux densities are on average higher than our flux densities by $\sim 20 \%$. In order to address this issue, we built radio spectra of $\sim 200$ radio sources using the $74 \mathrm{MHz}$ flux densities (that are in agreement with Cohen et al. (2003), see Sect. 4.3), and 1.4 GHz flux densities retrieved from the NVSS database. Also, we considered two more measurements obtained in August 2004 by the Giant Meterwave Radio Telescope

\footnotetext{
3 The largest multiple sources J0217.0-0449*, J0227.2-0325*, J0216.3-0245*, do not satisfy the $<60^{\prime \prime}$ criteria, but regarding at the morphology it is obvious that are actually multiple, see Fig. A.1.
} 

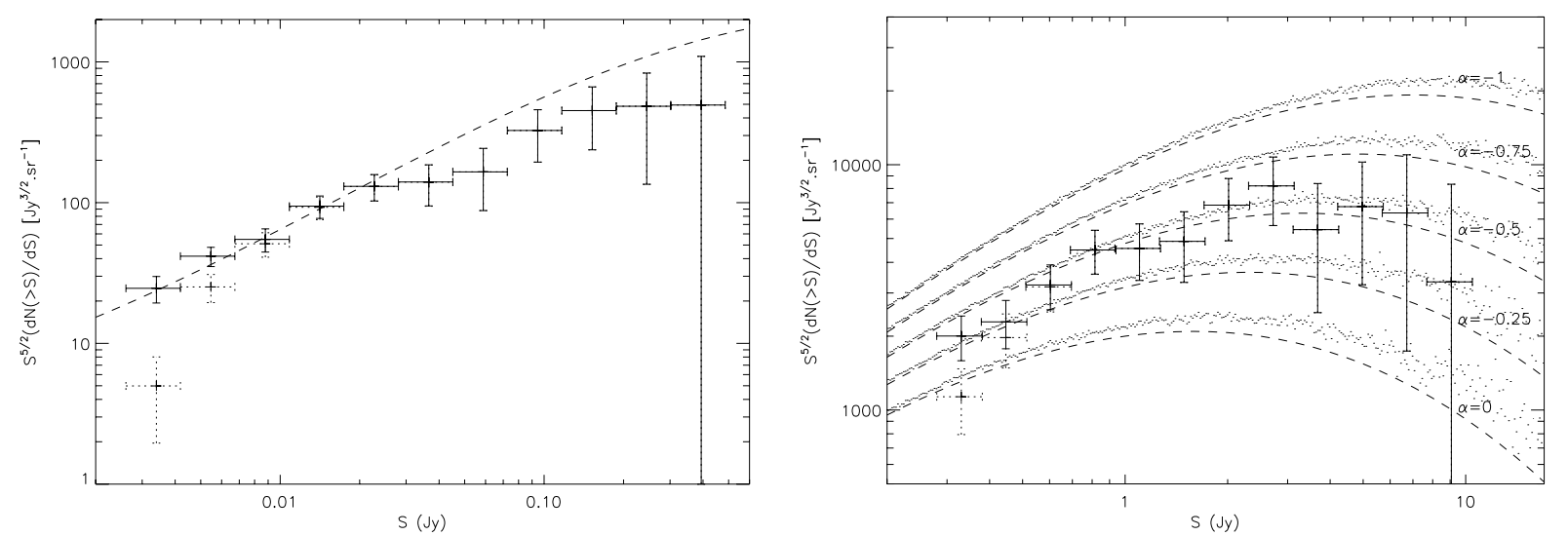

Fig. 7. The left panel shows the Euclidean normalized differential source count at $325 \mathrm{MHz}$. The values with doted error bar are uncorrected, whereas the error bar in full line show the differential source count corrected from the noise variations within the map and from the source finding algorithm efficiency falling steeply bellow $10 \sigma$. The differential source count from deep $325 \mathrm{MHz}$ survey (Wieringa 1991) is over plotted in dashed line. The right panel shows the Euclidean normalized differential source count at $74 \mathrm{MHz}$. The dashed lines shows the Wieringa (1991) differential source count extrapolated from 325 to $74 \mathrm{MHz}$ using different spectral index in the 0 to -1 range. Dots are showing the differential source count extrapolation derived from Monte-Carlo simulation, taking in account spectral index dispersion $\sigma\left(\alpha_{325}^{74}\right)=0.24$ of a typical radio source population (De Breuck et al. 2000).

(GMRT) at 230 and $610 \mathrm{MHz}$, covering the same field at comparable depth (Tasse et al. in prep.). Selecting a sample of single, unresolved sources at $325 \mathrm{MHz}$, most often, when the general trend of the radio spectra doesn't indicate any spectral aging or self absorption break, the flux density estimate by Cohen et al. (2003) is a poorer match to the physical synchrotron power low spectra, whereas the new flux density estimates and their associated error bars are well compatible with a power law spectra. We therefore conclude the flux densities published in Cohen et al. (2003) to be overestimated by $\sim 20 \%$, and the flux densities presented here to be more reliable. Although not understood, the overestimation on the fluxes of Cohen et al. (2003) might be due to instrumental or algorithm errors.

Figure 7 shows the Euclidean normalized differential source count. We have calculated the source density in each flux density bin as described in the previous section, compensating for noise inhomogeneities across the image. As the error bars have been computed considering the decreasing effective area, we claim that the derived source counts are correct down to the $5 \sigma$ level, where $\sigma$ is the minimum noise value in the noise map, so that $5 \sigma \sim 2.5 \mathrm{mJy} /$ beam. Comparing the Euclidean normalized differential source count estimation from a deep Westerbork survey (Wieringa 1991), we find good agreement at low flux densities, where we have applied the efficiency corrections, but at flux densities higher than $30 \mathrm{mJy}$, they appear to differ by a factor of $\sim 2-3$, which we attribute to either cosmic variance or resolution difference.

We have compared our source list to the NVSS database, looking for $1.4 \mathrm{GHz}$ counterparts within $45^{\prime \prime}$ of each source. The total integrated flux density used to derive the spectral index is calculated as described in Sect. 3.5. The resolution difference between our data (6.7') and NVSS (45"), makes the estimation of accurate spectral index less robust, as we can miss some of the extended emission seen in NVSS. This probably leads to a small over estimate of the spectral index for the extended sources, and we would need to match resolution to correct for this effect.

Out of the 847 detected sources at $325 \mathrm{MHz}$, we have found 566 sources to have NVSS counterparts, and for the 281 remaining sources we give an upper limit on their spectral index based on the NVSS detection limit. Figure 8 shows the spectral index distribution, and the corresponding flux density and completeness limit of NVSS. We have derived the median spectral index $\alpha_{325}^{1400} \sim-0.66$, for the whole radio source sample, excluding the unidentified sources in NVSS, and $\alpha_{325}^{1400}\left(S_{325}>\right.$ $0.05 \mathrm{Jy}) \sim-0.72$, on the brightest sources subsample. Figure 9 shows we find close agreement between the spectral index distributions of a $S>0.05$ Jy subsample, and the De Breuck et al. (2000) Gaussian fit to the spectral index distributions derived from the WENSS/NVSS surveys flux densities $\left(S_{325}>0.05 \mathrm{Jy}\right.$ subsample).

\subsection{A Radio halo candidate at $325 \mathrm{MHz}$}

Giant radio halo and relic radio sources are generally diffuse low surface brightness sources with steep spectra and typical physical sizes of $\sim 0.1-1 \mathrm{Mpc}$. They are found in rich environment, showing signs of cluster merger activity (for extended reviews on the subject see Feretti 1999; Sarazin 2005).

An extended, low surface brightness object of $\sim 1.9^{\prime}$ along the right ascension axis, and $\sim 0.9^{\prime}$ along the declination axis is detected at $325 \mathrm{MHz}$ at $\alpha(\mathrm{J} 2000)=2^{\mathrm{h}} 19^{\mathrm{m}} 42^{\mathrm{s}}, \delta(\mathrm{J} 2000)=$ $-4^{\circ} 00^{\prime} 30^{\prime \prime}$. Since that source is extended on the scale of the box being used for the local noise calculation, the peak flux density was below the $5 \sigma$ level after the local noise normalization. We have therefore extracted the Gaussian components of that source on the original map, which appear together with the other sources in Table A.1. Using AIPS's task TVSTAT, we find its integrated flux density to be $150.7 \pm 12.5 \mathrm{mJy}$. We detect the diffuse emission counterparts at $74 \mathrm{MHz}$ and $1.4 \mathrm{GHz}$ (NVSS, Condon et al. 1998) at flux density levels of $1.34 \pm 0.2 \mathrm{Jy}$, and $27.7 \pm 1.8 \mathrm{mJy}$ respectively. This makes the spectral indexes to be $\alpha_{74}^{325} \sim-1.48 \pm 0.15$ and $\alpha_{325}^{1400} \sim-1.16 \pm 0.1$. We have looked for counterparts using the NED databases (NED Team 1992), and Fig. 10 shows the overlay between an image retrieved in the Digital Sky Survey (DSS) and the radio halo contours. We have found four objects classified as galaxies in Maddox et al. (1990).

Based on the morphology and the fairly steep spectral index, we suggest that this object is a good candidate for either a radio halo, a radio relic or both. The difference between the spectral indexes $\alpha_{74}^{325}$ and $\alpha_{325}^{1400}$ suggests the presence of more than one electron population. The contour lines on the east side of the object shows a steep fall-off of the surface brightness, which 

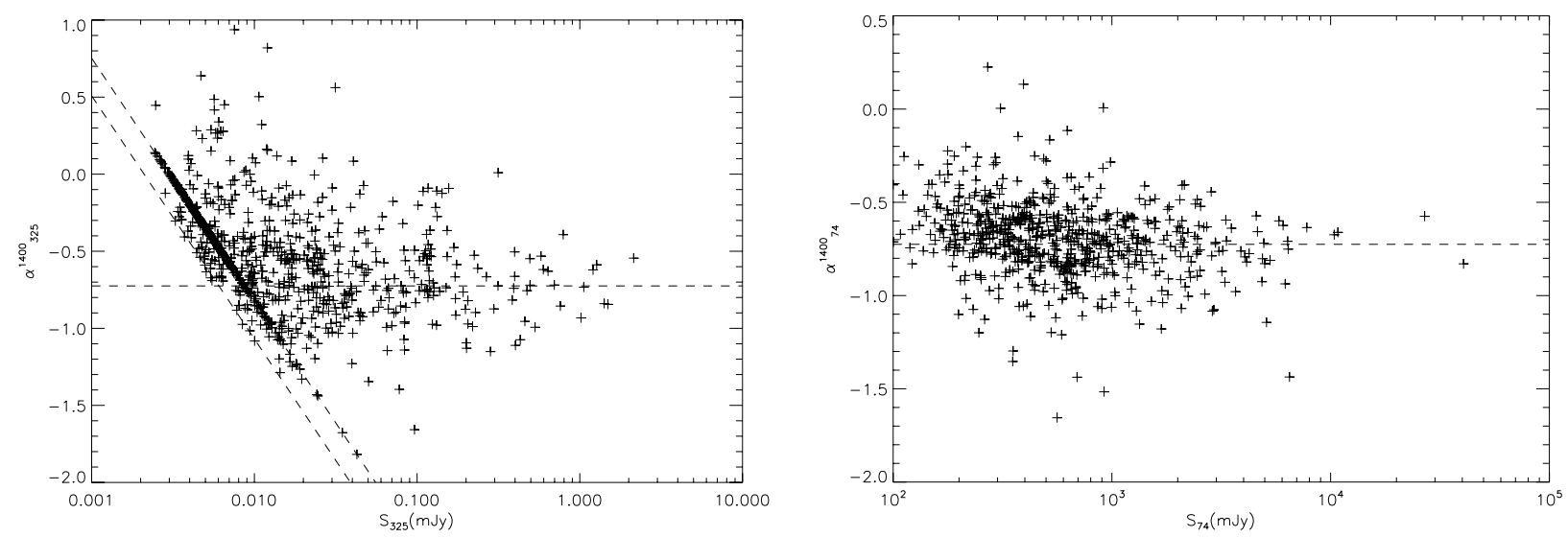

Fig. 8. Spectral index distribution at $325 \mathrm{MHz}$ (left panel) and $74 \mathrm{MHz}$ (right panel), derived from comparison with flux density of each radio source counterpart at $1.4 \mathrm{GHz}$ in the NVSS. The flat dashed lines on both plots represents the median value being respectively $\alpha_{325}^{1400}=-0.72$ and $\alpha_{74}^{1400}=-0.72$. In the $325 \mathrm{MHz}$ spectral index distribution plot, the dashed lines on the left correspond to the spectral index reachable as a function of the $325 \mathrm{MHz}$ flux density, with respect to the completeness, and flux density limit levels of NVSS.

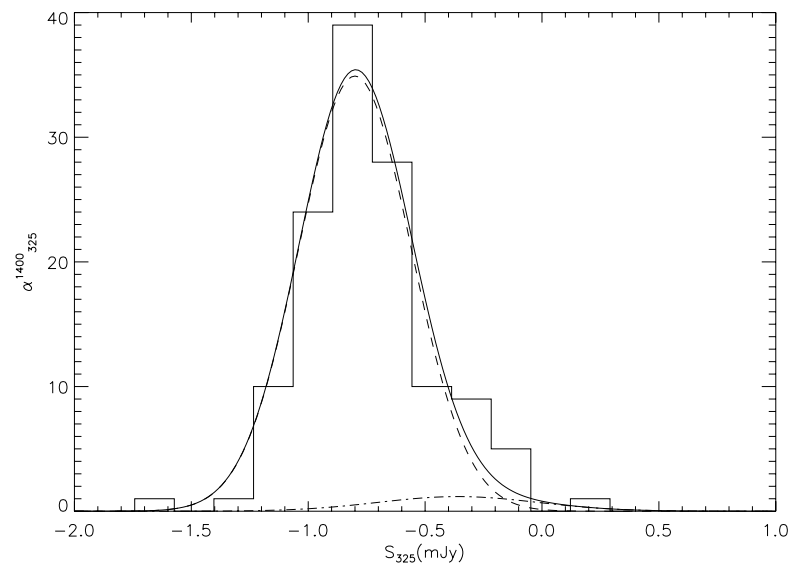

Fig. 9. Spectral index distribution $(S>0.05 \mathrm{Jy})$ comparison with De Breuck et al. (2000) Gaussian fits, based on the WENSS/NVSS surveys. Dashed line and dashed-dotted line show the spectral index distribution of the steep-spectrum and the flat-spectrum radio source population respectively (De Breuck et al. 2000).

indicates the presence of a shock. A polarization observation of the halo could confirm the relic origin of its diffuse emission. Visually, it looks like a galaxy overdensity and this diffuse emission likely belongs to a galaxy group or cluster as discovered in recent, similarly observed objects at low frequencies (Kassim et al. 2001). The detection of diffuse X-ray emission would confirm the cluster identification, but unfortunately, the $\sim 5$ degree $^{2}$ $\mathrm{X}$-ray field does not overlap the source.

\section{3. $74 \mathrm{MHz}$ results}

At $74 \mathrm{MHz}$, on the criteria outlined above, we detect 686 sources. Matching the source list with NVSS $1.4 \mathrm{GHz}$ objects, we find 20 sources to be false detections due to the correlated sidelobe noise close to bright sources. As for the $325 \mathrm{MHz}$ data, any sources closer than $60^{\prime \prime}$ have been classified as multiple. Of the 666 remaining sources, 615 have been classified as single (S) and 51 as multiple (M) (see Fig. A.2). Of the 615 simple sources, 465 were unresolved. Yet, as discussed in Sect. 3, the size measurement at $74 \mathrm{MHz}$ have very high uncertainties, as the seeing effect is poorly defined.

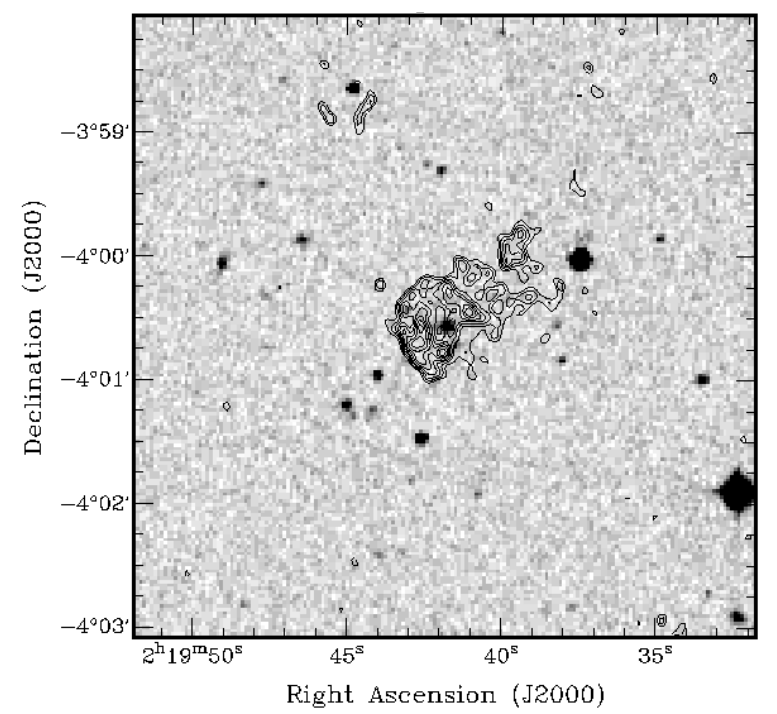

Fig. 10. Radio contours of the giant radio halo candidate detected at $325 \mathrm{MHz}$ overlaid with an image retrieved from Digitized Sky Survey. The contours corresponds to levels of $1.5 \mathrm{mJy} /$ Beam $\times\{1,1.4,2,2.8 \ldots\}$.

As for the $325 \mathrm{MHz}$ dataset, we have checked for the consistency between our and Cohen et al. (2003) observed flux densities at $74 \mathrm{MHz}$. Results do not show any significant overall offsets in between the two measurement, as opposed to the $325 \mathrm{MHz}$ observations. In addition, we compare our flux density estimates to the VLSS radio survey at $74 \mathrm{MHz}$ (Cohen et al. 2006). Again, flux differences are compatible with the error bar estimates.

At $74 \mathrm{MHz}$, assuming $\alpha=-0.8$ the corresponding flux density limit at $1.4 \mathrm{GHz}$ is about an order of magnitude higher than the NVSS flux density limit. Consequently, all the $74 \mathrm{MHz}$ sources have a counterpart in the NVSS database within a $60^{\prime \prime}$ radius. Moreover our $30^{\prime \prime}$ resolution $74 \mathrm{MHz}$ map roughly matching the NVSS resolution of $45^{\prime \prime}$, we expect the spectral index estimation to be highly reliable. The spectral index distribution is shown in Fig. 8, and we find a spectral index median value of $\alpha_{74}^{1400}=-0.72$.

Figure 7 shows the Euclidean normalized differential source count with and without applied corrections. In order to compare that result, we can analytically extrapolate the relation given 
Table 3. Objects identified in NED database with known redshifts.

\begin{tabular}{|c|c|c|c|c|c|c|c|c|}
\hline Name & $\begin{array}{l}\text { RA radio source } \\
(\mathrm{J} 2000)\end{array}$ & $\begin{array}{l}\text { Dec radio rource } \\
(\mathrm{J} 2000)\end{array}$ & Type & $\begin{array}{c}\text { RA } \\
\text { NED object }\end{array}$ & $\begin{array}{c}\text { Dec } \\
\text { NED object }\end{array}$ & $\begin{array}{l}\text { Dist from } \\
\text { object (") }\end{array}$ & Redshift & Reference \\
\hline J0201.7-0211 & 020143.10 & -021151.93 & $\mathrm{G}$ & 020143.11 & -021147.6 & 4.33 & 0.19590 & 2 \\
\hline J0201.7-0211 & 020147.18 & -021159.60 & G & 020147.03 & -021155.7 & 4.50 & $0.19600 \pm 0.00100$ & 3 \\
\hline J0213.7-0256 & 021346.93 & -025641.32 & G & 021347.00 & -025637.5 & 3.96 & $0.35680 \pm 0.00020$ & 4 \\
\hline J0215.6-0222* & 021541.85 & -022254.27 & QSO & 021542.02 & -022256.8 & 3.59 & 1.17800 & 5 \\
\hline $\mathrm{J} 0216.2+0008$ & 021616.51 & +000859.32 & GClstr & 021617.30 & +00 1137.0 & 158.12 & 0.21998 & 1 \\
\hline J0216.6-0444* & 021640.90 & -044405.12 & QSO & 021640.65 & -044404.7 & 3.77 & 0.87000 & 6 \\
\hline J0217.4-0015 & 021725.01 & -001547.02 & GClstr & 021732.80 & -001552.0 & 116.95 & 0.31075 & 1 \\
\hline J0218.5-0923 & 021834.50 & -092339.64 & QSO & 021834.38 & -09 2338.5 & 2.13 & 0.47000 & 6 \\
\hline J0218.6-0015 & 021839.78 & -001507.11 & GClstr & 021839.40 & -001220.0 & 167.20 & 0.31075 & 1 \\
\hline J0220.0-0143 & 022001.98 & -014349.67 & QSO & 022002.31 & -014352.7 & 5.80 & 0.47000 & 6 \\
\hline $\mathrm{J} 0220.5+0027$ & 022032.50 & +00 2759.65 & GClstr & 022034.30 & +00 2756.0 & 27.24 & 0.26537 & 1 \\
\hline J0220.9-0156* & 022054.11 & -015653.07 & G & 022054.25 & -015651.8 & 2.45 & 0.17500 & 7 \\
\hline J0223.0-0826 & 022301.84 & -082609.02 & QSO & 022301.55 & -082609.9 & 4.43 & 1.52070 & 8 \\
\hline J0224.3-0752 & 022422.41 & -075258.85 & QSO & 022422.39 & -075258.7 & 0.33 & 2.44920 & 8 \\
\hline $\mathrm{J} 0225.1-0035$ & 022507.73 & -003532.50 & QSO & 022508.09 & -003531.4 & 5.51 & 0.68700 & 9 \\
\hline \multirow[t]{2}{*}{ J0227.6-0052 } & 022736.20 & -005258.03 & GClstr & 022735.40 & -005405.0 & 68.03 & 0.34479 & 1 \\
\hline & & & GClstr & 022735.50 & -005132.0 & 86.66 & 0.33344 & 1 \\
\hline $\mathrm{J} 0228.4+0032$ & 022825.01 & +003212.08 & GClstr & 022826.50 & +003220.0 & 23.71 & 0.50000 & 10 \\
\hline J0228.6-0042 & 022840.90 & -004255.03 & GClstr & 022842.90 & -004320.0 & 39.03 & 0.42421 & 1 \\
\hline \multirow[t]{2}{*}{ J0228.1-0115 } & 022807.50 & -011543.10 & AbLS & 022807.79 & -011540.5 & 5.06 & 1.99760 & 11 \\
\hline & & & QSO & 022807.80 & -011540.6 & 5.14 & 2.03700 & 1 \\
\hline $\mathrm{J} 0230.4+0108$ & 023026.25 & +010849.10 & GClstr & 023027.40 & +010904.0 & 22.79 & 0.40000 & 10 \\
\hline J0231.0-0049 & 023100.82 & -004944.42 & GClstr & 023111.20 & -004921.0 & 157.45 & 0.39017 & 1 \\
\hline \multirow{2}{*}{ J0233.4+0015 } & 023325.37 & +001548.75 & GClstr & 023328.10 & +00 1722.0 & 101.84 & 0.35613 & 1 \\
\hline & & & GClstr & 023327.90 & +00 1837.0 & 172.47 & 0.34479 & 1 \\
\hline J0233.5-0203 & 023330.17 & -020322.19 & G & 023330.34 & -020322.4 & 2.55 & 0.79400 & 9 \\
\hline J0234.3-0139 & 023421.64 & -013900.25 & G & 023421.83 & -013900.6 & 2.87 & $0.64500 \pm 0.00200$ & 12 \\
\hline J0234.9-0736 & 023458.71 & -073617.98 & QSO & 023458.44 & -073619.8 & 4.44 & 2.17260 & 8 \\
\hline J0235.5-0705 & 023530.73 & -070501.43 & QSO & 023530.71 & -070504.6 & 3.18 & 2.05980 & 8 \\
\hline J0235.5-0219 & 023532.43 & -021931.19 & QSO & 023532.51 & -021932.0 & 1.44 & 1.32100 & 9 \\
\hline J0237.9-0145 & 023757.00 & -014510.77 & $\mathrm{G}$ & 023757.09 & -014511.4 & 1.48 & 0.84000 & 9 \\
\hline J0239.2-0118 & 023913.42 & -011815.01 & QSO & 023913.68 & -011816.4 & 4.14 & 1.79400 & 9 \\
\hline J0239.7-0234 & 023945.71 & -023439.93 & QSO & 023945.47 & -023440.9 & 3.72 & 1.11600 & 9 \\
\hline \multirow[t]{2}{*}{ J0242.6-0000 } & 024240.40 & -000045.21 & $\mathrm{G}$ & 024240.71 & -000047.8 & 5.32 & $(1137 \pm 3) \mathrm{km} \mathrm{s}^{-1}$ & 13 \\
\hline & & & GClstr & 024232.10 & +00 0014.0 & 137.86 & 0.21998 & 1 \\
\hline J0242.7-0157 & 024247.57 & -015746.54 & QSO & 024247.65 & -015749.6 & 3.28 & 0.61700 & 9 \\
\hline
\end{tabular}

An asterisk (*) after the source-name indicates the source has been detected at both frequencies.

References: (1) Goto et al. (2002), (2) Crawford et al. (1999), (3) Crawford et al. (1995), (4) Owen et al. (1995), (5) Drinkwater et al. (1997), (6) Becker et al. (2001), (7) Hewitt \& Burbidge (1991), (8) Schneider et al. (2003), (9) Dunlop et al. (1989), (10) Postman et al. (1996), (11) Junkkarinen et al. (1991), (12) Stanford et al. (2000), (13) Huchra et al. (1999).

by Wieringa (1991) from $325 \mathrm{MHz}$ to $74 \mathrm{MHz}$, assuming various mean spectral indices $\left(\left\langle\alpha_{74}^{325}\right\rangle=\{-1.00,-0.75,-0.50\right.$, $-0.25,0.00\})$. Also, to take in account the spectral index dispersion of the radio source population at $325 \mathrm{MHz}$, we have conducted an extensive Monte-Carlo simulation, by generating a radio source population following the Wieringa (1991) source counts at $325 \mathrm{MHz}$, and then giving each source a random spectral index following a Gaussian distribution with mean values of $\left\langle\alpha_{74}^{325}\right\rangle$, and a dispersion of $\sigma\left(\alpha_{74}^{325}\right)=0.24$ (De Breuck et al. 2000). Extrapolating the flux density of each source to $74 \mathrm{MHz}$, we can build the differential source count at $74 \mathrm{MHz}$. Figure 7 shows that the analytical and Monte-Carlo extrapolated differential source counts are in close agreement.

The Euclidean normalized differential source count of our sample roughly corresponds to the extrapolation done with $\alpha_{74}^{325} \sim-0.5$ which contrasts with the median value $\alpha_{74}^{1400}=$ -0.72 found between our $74 \mathrm{MHz}$ sources and their NVSS counterparts at $1.4 \mathrm{GHz}$. This suggests a flattening of the spectrum at $v<325 \mathrm{MHz}$, likely due to the sources being synchrotron self absorbed.

\subsection{Source identification from literature:}

We have searched for published data on all objects we have detected at both frequencies, using the NASA/IPAC Extragalactic database (NED / NED Team 1992). We set the searching radius at $6^{\prime \prime}$ for the individual object search, and at $3^{\prime}$ for the galaxy cluster search. On the 1460 detected objects at 74 and $325 \mathrm{MHz}$, 34 with known redshift have been identified optically as QSO, Galaxy, or are expected to belong to a galaxy cluster, and we have only selected the ones with their redshift determined (see Table 3). All of them have been identified at $74 \mathrm{MHz}$ and only four have also been identified at $325 \mathrm{MHz}$. This appears to be a selection effect as the survey at $74 \mathrm{MHz}$ probes a much brighter source population and an area which is $\sim 7$ times larger than at $325 \mathrm{MHz}$. Figure 11 shows the redshift distribution of our identified source sub-sample.

\section{Conclusion and future work}

We have mapped the XMM-LSS field over 130 and $\sim 15$ squares degrees at 74 and $325 \mathrm{MHz}$ respectively, 


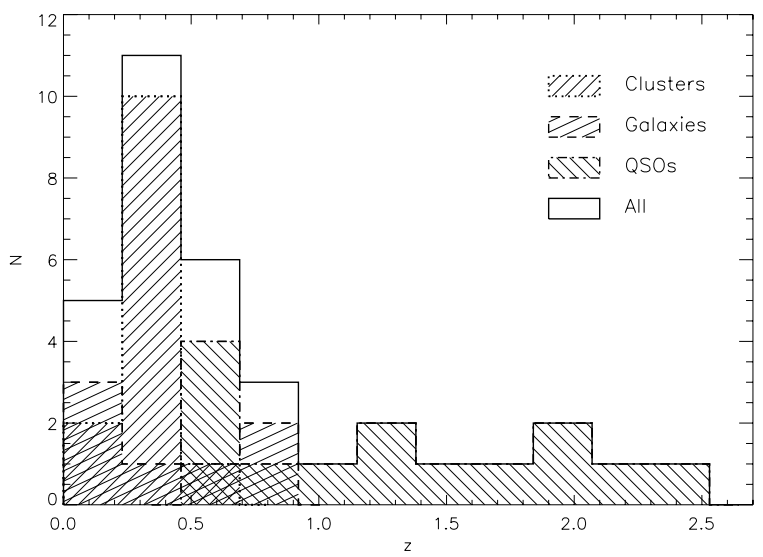

Fig. 11. Redshift distribution of our whole radio source sample identified with NED, and with measured redshift for the QSOs, galaxies, and galaxy cluster.

detecting $\sim 1500$ sources in total. We detect one source of diffuse, steep-spectrum emission, which is a candidate for a radio halo or relic. The Euclidean normalized differential source counts at $74 \mathrm{MHz}$ are consistent with previous studies assuming $\alpha_{325}^{74} \sim-0.5$, suggesting a flattening of the spectrum of radio source population for $v<325 \mathrm{MHz}$.

In the near future we will combine the VLA data at 74 and $325 \mathrm{MHz}$ with observations from the GMRT (Giant Meterwave Radio Telescope) at 230 and $610 \mathrm{MHz}$ of the XMM-LSS field, adding two additional frequencies. Crosscorrelating this data with upcoming X-ray and optical observations will allow us to probe in detail the low frequency spectrum of a large radio galaxy sample, and to determine the influence of the small and large scale environment on the radio source properties, such as linear size, and radio power.

Acknowledgements. The authors have made use of the NASA/IPAC Extragalactic Database (NED), which is operated by the Jet Propulsion Laboratory, Caltech, under contract with the National Aeronautics and Space administration. Basic research in radio astronomy at the Naval Research Laboratory is funded by the Office of Naval Research. The authors thank the referee for a number of helpful suggestions.

\section{References}

Becker, R. H., White, R. L., Gregg, M. D., et al. 2001, ApJS, 135, 227 Best, P. N., Kauffmann, G., Heckman, T. M., et al. 2005, MNRAS, 362, 25 Blanton, E. L., Sarazin, C. L., McNamara, B. R., \& Clarke, T. E. 2004, ApJ, 612, 817
Bondi, M., Ciliegi, P., Zamorani, G., et al. 2003, A\&A, 403, 857

Cohen, A. S., Röttgering, H. J. A., Kassim, N. E., et al. 2003, ApJ, 591, 640

Cohen, A. S., Lane, W. M., Kassim, N. E., et al. 2006, in ASP Conf. Ser., 299

Condon, J. J. 1997, PASP, 109, 166

Condon, J. J., Cotton, W. D., Greisen, E. W., et al. 1998, AJ, 115, 1693

Cotton, W. D., Condon, J. J., Perley, R. A., et al. 2004, in Proc. SPIE, 5489, 180

Crawford, C. S., Allen, S. W., Ebeling, H., Edge, A. C., \& Fabian, A. C. 1999, MNRAS, 306, 857

Crawford, C. S., Edge, A. C., Fabian, A. C., et al. 1995, MNRAS, 274, 75

Croston, J. H., Hardcastle, M. J., Birkinshaw, M., \& Worrall, D. M. 2005, in X-Ray and Radio Connections, ed. L. O. Sjouwerman, \& K. K. Dyer, Published electronically by NRAO, http://www.aoc.nrao.edu/ events/xraydio Held 3-6 February 2004 in Santa Fe, New Mexico, USA, (E7.06) 7 pages

De Breuck, C., van Breugel, W., Röttgering, H. J. A., \& Miley, G. 2000, A\&AS, 143,303

Drinkwater, M. J., Webster, R. L., Francis, P. J., et al. 1997, MNRAS, 284, 85

Dunlop, J. S., Peacock, J. A., Savage, A., et al. 1989, MNRAS, 238, 1171

Fabian, A. C., Sanders, J. S., Allen, S. W., et al. 2003, MNRAS, 344, L43

Fabian, A. C., Sanders, J. S., Taylor, G. B., \& Allen, S. W. 2005, MNRAS, 360, L20

Fanaroff, B. L., \& Riley, J. M. 1974, MNRAS, 167, 31P

Feretti, L. 1999, in Diffuse Thermal and Relativistic Plasma in Galaxy Clusters, 3

Goto, T., Sekiguchi, M., Nichol, R. C., et al. 2002, AJ, 123, 1807

Hewitt, A., \& Burbidge, G. 1991, ApJS, 75, 297

Hill, G. J., \& Lilly, S. J. 1991, ApJ, 367, 1

Huchra, J. P., Vogeley, M. S., \& Geller, M. J. 1999, ApJS, 121, 287

Junkkarinen, V., Hewitt, A., \& Burbidge, G. 1991, ApJS, 77, 203

Kassim, N. E., Perley, R. A., Erickson, W. C., \& Dwarakanath, K. S. 1993, AJ, 106,2218

Kassim, N. E., Clarke, T. E., Enßlin, T. A., Cohen, A. S., \& Neumann, D. M. 2001, ApJ, 559, 785

Ledlow, M. J., \& Owen, F. N. 1996, AJ, 112, 9

Lonsdale, C. J., Smith, H. E., Rowan-Robinson, M., et al. 2003, PASP, 115, 897 Maddox, S. J., Efstathiou, G., Sutherland, W. J., \& Loveday, J. 1990, MNRAS, 243, 692

McCarthy, P. J. 1993, ARA\&A, 31, 639

Owen, F. N., Ledlow, M. J., \& Keel, W. C. 1995, AJ, 109, 14

Perley, R. A. 1999, in Synthesis Imaging in Radio Astronomy II, ASP Conf. Ser., 180,383

Pierre, M., Valtchanov, I., Altieri, B., et al. 2004, J. Cosmol. Astro-Part. Phys., 9,11

Postman, M., Lubin, L. M., Gunn, J. E., et al. 1996, AJ, 111, 615

Refregier, A., Valtchanov, I., \& Pierre, M. 2002, A\&A, 390, 1

Rengelink, R. B., Tang, Y., de Bruyn, A. G., et al. 1997, A\&AS, 124, 259

Sarazin, C. L. 2005, in X-Ray and Radio Connections, ed. L. O. Sjouwerman, \& K. K. Dyer, Published electronically by NRAO, http://www. aoc.nrao.edu/events/xraydio Held 3-6 February 2004 in Santa Fe, New Mexico, USA, (E8.00) 3 pages

Schneider, D. P., Fan, X., Hall, P. B., et al. 2003, AJ, 126, 2579

Stanford, S. A., Stern, D., van Breugel, W., \& De Breuck, C. 2000, ApJS, 131, 185

Wieringa, M. H. 1991, Ph.D. Thesis

Yates, M. G., Miller, L., \& Peacock, J. A. 1989, MNRAS, 240, 129

Zirbel, E. L., \& Baum, S. A. 1995, ApJ, 448, 521 
C. Tasse et al.: The low-frequency counterpart of the XMM large scale structure survey, Online Material p 1

\section{Online Material}


C. Tasse et al.: The low-frequency counterpart of the XMM large scale structure survey, Online Material $p 2$

\section{Appendix A: Radio images and source lists}
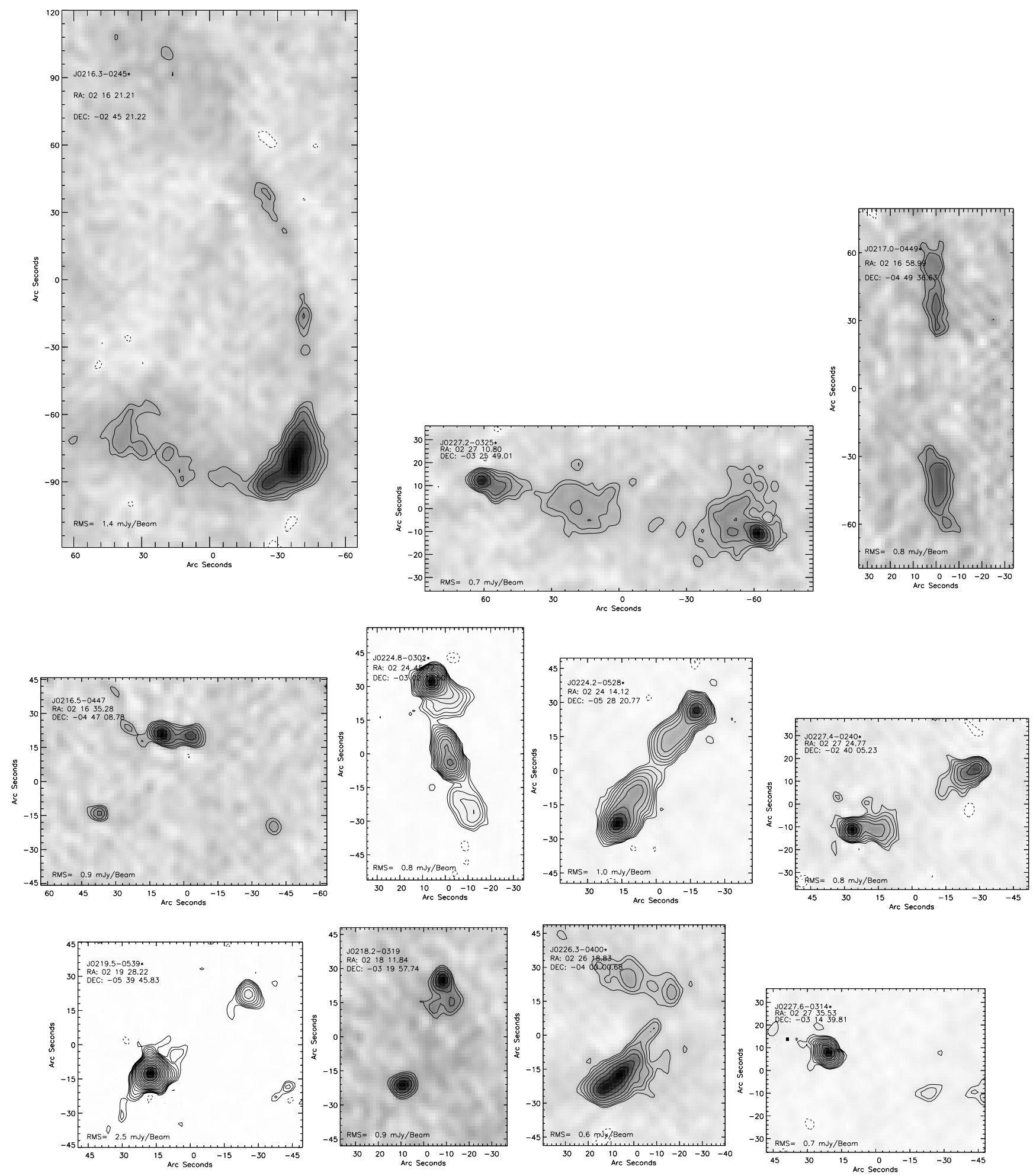

Fig. A.1. The multiple component sources in the $325 \mathrm{MHz}$ source list larger than 13", sorted in decreasing angular size order. Contours corresponds to levels of $3 \sigma \times(-1.4,-1,1,1.4,2,2.8,4,5.6,8,11, \ldots)$ and greyscale is scaled from $-3 \sigma$ to the maximum value in the image. On the top-left corner of each images appears the name of the corresponding source in the source list, as well as its mean coordinate. The local noise level is shown on the bottom of each image. 
C. Tasse et al.: The low-frequency counterpart of the XMM large scale structure survey, Online Material p 3
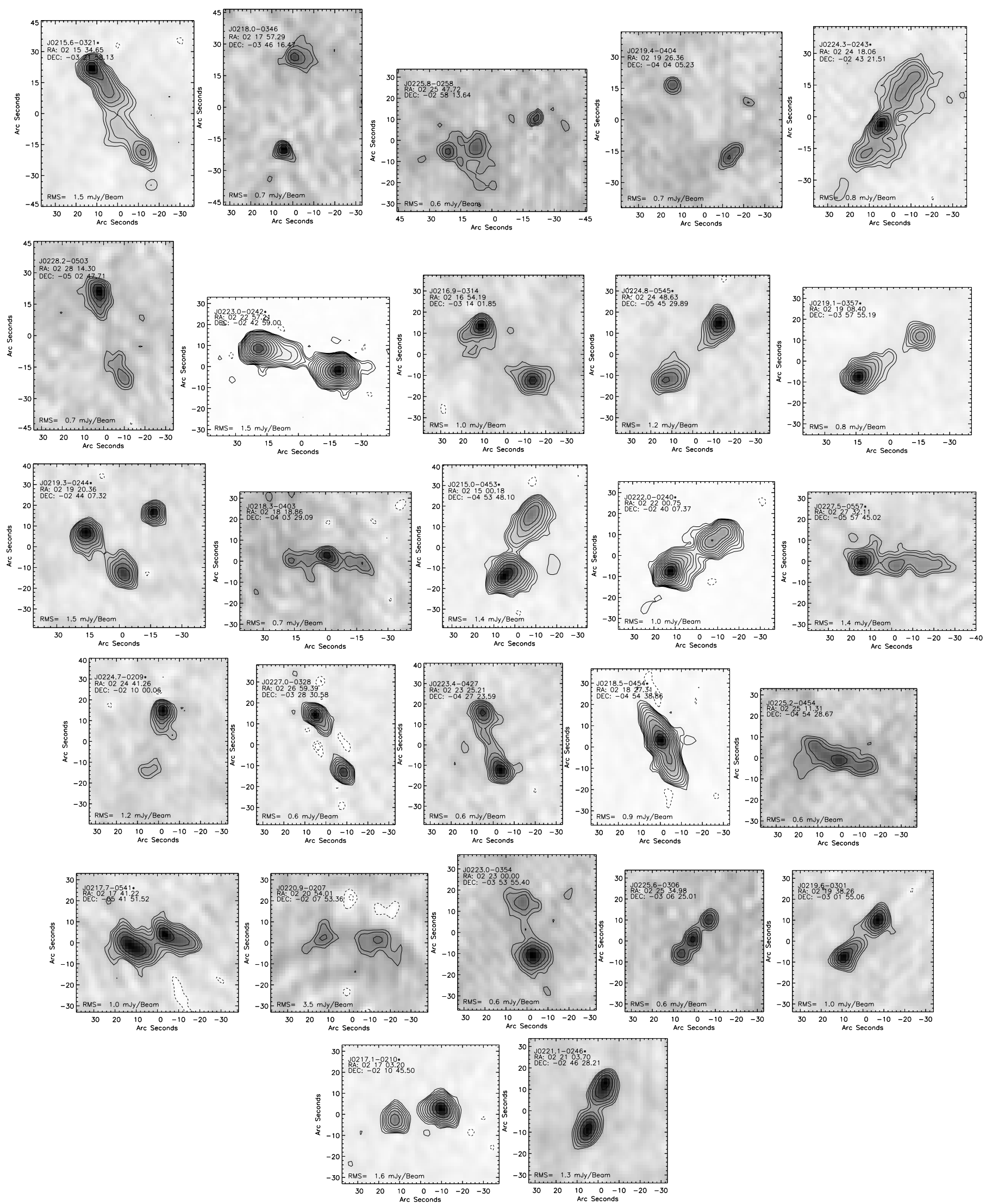

Fig. A.1. continued. 
C. Tasse et al.: The low-frequency counterpart of the XMM large scale structure survey, Online Material p 4
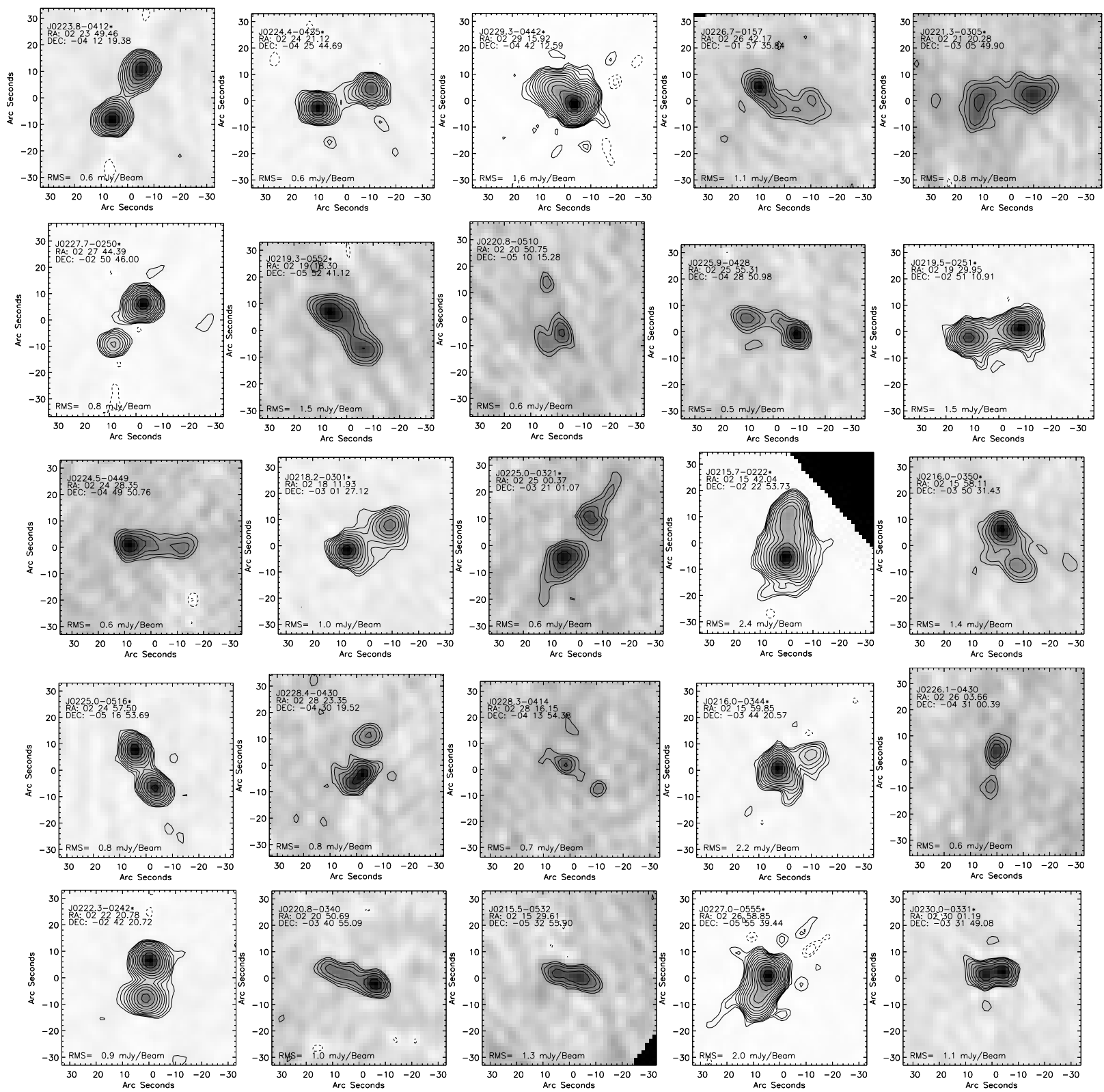

Fig. A.1. continued. 
C. Tasse et al.: The low-frequency counterpart of the XMM large scale structure survey, Online Material p 5
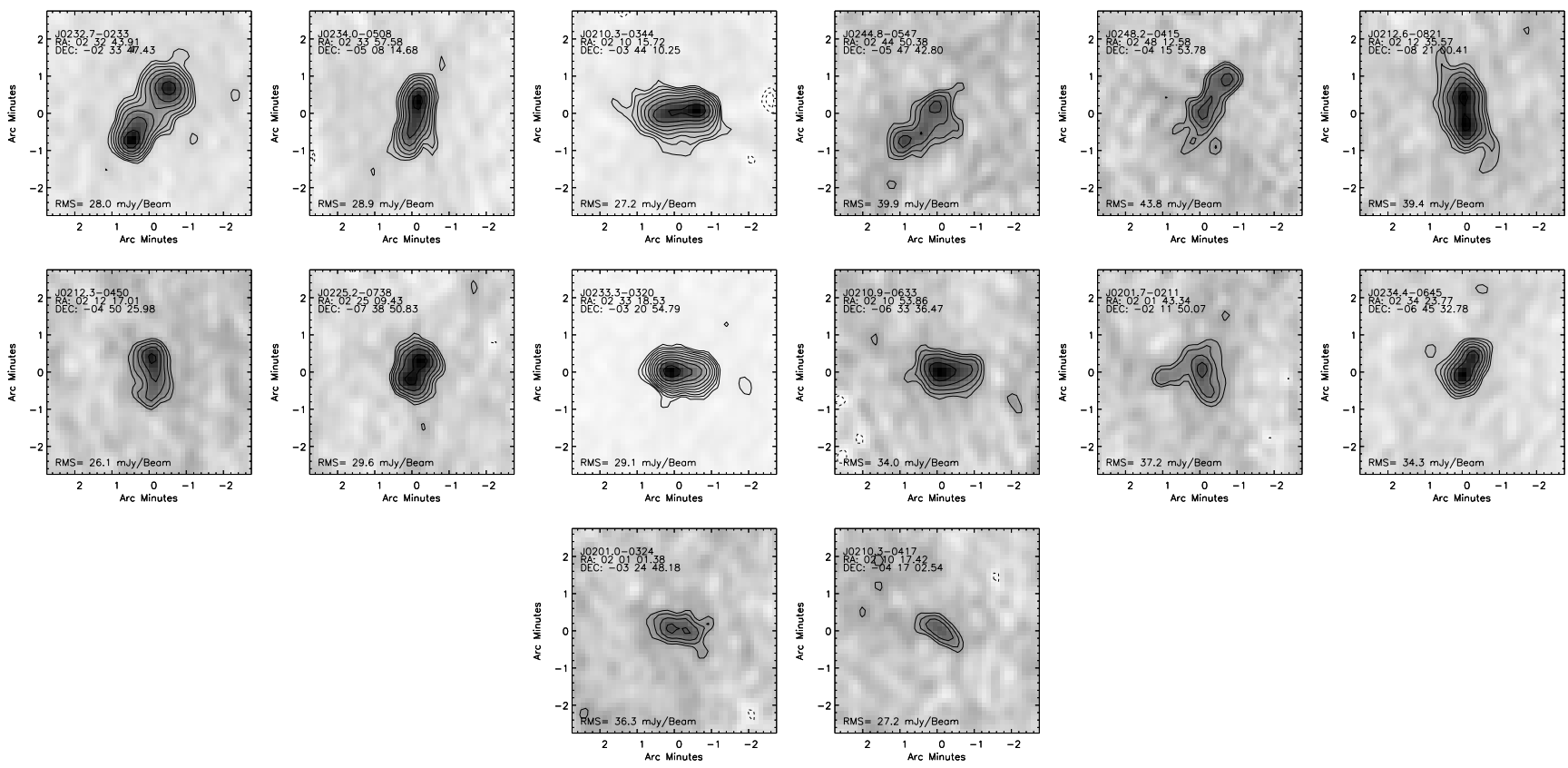

Fig. A.2. The multiple component sources in the $74 \mathrm{MHz}$ source list larger than $60^{\prime \prime}$, sorted in decreasing angular size order. Contours corresponds to levels of $3 \sigma \times(-1.4,-1,1,1.4,2,2.8,4,5.6,8,11, \ldots)$ and greyscale is scaled from $-3 \sigma$ to the maximum value in the image. On the top-left corner of each images appears the name of the corresponding source in the source list, as well as its mean coordinate. The local noise level is shown on the bottom of each image. 
C. Tasse et al.: The low-frequency counterpart of the XMM large scale structure survey, Online Material p 6

Table A.1. The $325 \mathrm{MHz}$ source list.

\begin{tabular}{|c|c|c|c|c|c|c|c|c|c|c|c|}
\hline Name & $\begin{array}{c}\text { RA } \\
\text { J2000 }\end{array}$ & $\begin{array}{c}\sigma_{\alpha} \\
\operatorname{arcsec}\end{array}$ & $\begin{array}{c}\text { Dec } \\
\text { J2000 }\end{array}$ & $\begin{array}{c}\sigma_{\delta} \\
\operatorname{arcsec}\end{array}$ & Type & $\begin{array}{l}S_{\text {int }} \\
\text { mJy }\end{array}$ & $\begin{array}{c}S_{\text {comp }} \\
\text { mJy }\end{array}$ & $\begin{array}{c}\text { Major axis } \\
\text { arcsec }\end{array}$ & $\begin{array}{c}\text { Minor axis } \\
\text { arcsec }\end{array}$ & $\begin{array}{c}\text { Position } \\
\text { Angle }\end{array}$ & $\begin{array}{c}\text { Spectral } \\
\text { index } \alpha_{325}^{1400}\end{array}$ \\
\hline \multirow[t]{2}{*}{ J0214.5-0314 } & 021431.89 & 2.47 & -031427.12 & 1.77 & $M$ & $77.8 \pm 7.7$ & $49.8 \pm 7.1$ & $33.0 \pm 10.8$ & $<3.1$ & $85.2 \pm 0.9$ & -1.40 \\
\hline & 021431.90 & 1.07 & -031423.56 & 1.75 & $C$ & & $27.9 \pm 3.1$ & $<2.9$ & $<2.7$ & $156.3 \pm 41.2$ & \\
\hline J0214.6-0333 & 021434.07 & 1.37 & -033342.48 & 1.90 & $S$ & $7.0 \pm 1.6$ & $7.0 \pm 1.6$ & $<9.7$ & $<5.1$ & $51.2 \pm 10.3$ & -0.47 \\
\hline \multirow[t]{2}{*}{$\mathrm{J} 0214.7-0314$} & 021442.70 & 1.07 & -031420.43 & 1.75 & $M$ & $55.8 \pm 4.9$ & $37.2 \pm 4.0$ & $<3.0$ & $<2.5$ & $144.3 \pm 8.6$ & -0.50 \\
\hline & 021442.48 & 1.16 & -031423.31 & 1.81 & $C$ & & $18.6 \pm 2.9$ & $6.5 \pm 4.5$ & $<4.0$ & $45.5 \pm 7.1$ & \\
\hline \multirow[t]{2}{*}{ J0214.9-0411 } & 021452.88 & 1.08 & -041121.00 & 1.76 & $M$ & $22.6 \pm 2.3$ & $13.0 \pm 1.8$ & $<4.1$ & $<3.3$ & $125.8 \pm 20.1$ & -0.37 \\
\hline & 021452.70 & 1.15 & -041121.04 & 1.85 & $C$ & & $9.6 \pm 1.6$ & $<7.6$ & $<3.2$ & $37.1 \pm 4.3$ & \\
\hline \multirow[t]{2}{*}{ J0214.9-0324 } & 021453.43 & 1.08 & -032457.64 & 1.75 & $M$ & $19.4 \pm 2.1$ & $11.1 \pm 1.5$ & $<3.9$ & $<2.8$ & $99.9 \pm 10.3$ & -0.97 \\
\hline & 021453.48 & 1.32 & -032500.67 & 1.76 & $C$ & & $8.3 \pm 1.4$ & $<8.0$ & $<3.2$ & $81.6 \pm 4.2$ & \\
\hline J0214.9-0451 & 021455.07 & 1.12 & -045127.92 & 1.99 & $S$ & $6.4 \pm 1.3$ & $6.4 \pm 1.3$ & $<9.0$ & $<4.5$ & $165.5 \pm 8.3$ & $<-0.52$ \\
\hline J0214.9-0249 & 021455.46 & 1.07 & -024919.56 & 1.76 & $S$ & $31.3 \pm 3.5$ & $31.3 \pm 3.5$ & $<3.1$ & $<2.8$ & $164.0 \pm 18.5$ & -0.50 \\
\hline J0214.9-0425 & 021456.03 & 1.21 & -042543.28 & 1.81 & $S$ & $7.5 \pm 1.4$ & $7.5 \pm 1.4$ & $<7.2$ & $<4.8$ & $55.5 \pm 14.4$ & -0.48 \\
\hline J0215.0-0256 & 021457.42 & 1.11 & -025602.19 & 1.77 & $S$ & $14.3 \pm 2.0$ & $14.3 \pm 2.0$ & $<4.9$ & $<4.4$ & $65.0 \pm 42.7$ & -0.47 \\
\hline \multirow[t]{3}{*}{ J0215.0-0458* } & 021458.92 & 1.06 & -045812.87 & 1.76 & $M$ & $121.6 \pm 8.6$ & $62.4 \pm 6.5$ & $6.5 \pm 1.1$ & $<1.8$ & $33.1 \pm 1.5$ & -0.49 \\
\hline & 021459.11 & 1.06 & -045813.16 & 1.75 & $C$ & & $51.4 \pm 5.4$ & $3.2 \pm 1.2$ & $<2.0$ & $140.5 \pm 3.6$ & \\
\hline & 021458.92 & 1.18 & -045741.24 & 1.89 & $C$ & & $7.8 \pm 1.6$ & $<8.1$ & $<4.7$ & $33.0 \pm 11.2$ & \\
\hline \multirow[t]{5}{*}{ J0215.0-0453* } & 021500.37 & 1.06 & -045359.65 & 1.75 & $M$ & $392.5 \pm 25.8$ & $224.0 \pm 22.7$ & $11.6 \pm 0.6$ & $5.3 \pm 0.4$ & $133.8 \pm 1.3$ & -0.74 \\
\hline & 021459.63 & 1.08 & -045330.22 & 1.78 & $C$ & & $83.5 \pm 9.0$ & $14.3 \pm 1.8$ & $<2.5$ & $148.6 \pm 1.4$ & \\
\hline & 021459.32 & 1.10 & -045330.90 & 1.86 & $C$ & & $55.9 \pm 7.2$ & $14.4 \pm 3.3$ & $5.1 \pm 2.0$ & $163.0 \pm 4.2$ & \\
\hline & 021459.78 & 1.15 & -045401.06 & 1.86 & $C$ & & $19.6 \pm 3.2$ & $8.0 \pm 4.3$ & $<5.0$ & $152.7 \pm 11.3$ & \\
\hline & 021500.06 & 1.30 & -045341.64 & 1.81 & $C$ & & $9.6 \pm 1.8$ & $<7.8$ & $<5.9$ & $92.2 \pm 23.3$ & \\
\hline J0215.0-0506 & 021502.03 & 1.13 & -050601.65 & 1.79 & $M$ & $116.6 \pm 9.2$ & $74.6 \pm 8.2$ & $17.0 \pm 2.6$ & $<2.2$ & $46.9 \pm 0.9$ & -0.80 \\
\hline & 021501.94 & 1.08 & -050605.33 & 1.76 & $C$ & & $33.4 \pm 3.9$ & $5.4 \pm 1.8$ & $<3.3$ & $105.0 \pm 10.7$ & \\
\hline & 021502.50 & 1.14 & -050600.96 & 1.76 & $C$ & & $8.6 \pm 1.4$ & $<5.6$ & $<3.5$ & $72.3 \pm 11.1$ & \\
\hline J0215.1-0521 & 021504.00 & 1.07 & -052139.18 & 1.75 & $M$ & $47.2 \pm 4.4$ & $27.3 \pm 3.0$ & $<3.0$ & $<2.3$ & $50.9 \pm 7.3$ & -0.66 \\
\hline & 021503.73 & 2.05 & -052143.20 & 1.81 & $C$ & & $19.9 \pm 3.2$ & $14.6 \pm 9.7$ & $<2.5$ & $76.0 \pm 1.1$ & \\
\hline J0215.1-0304 & 021505.12 & 1.20 & -030421.83 & 1.79 & $S$ & $10.0 \pm 1.8$ & $10.0 \pm 1.8$ & $<6.6$ & $<5.1$ & $74.5 \pm 21.3$ & -1.08 \\
\hline J0215.1-0322 & 021506.41 & 1.14 & -032233.66 & 2.39 & $S$ & $16.5 \pm 3.4$ & $16.5 \pm 3.4$ & $13.0 \pm 9.2$ & $<4.8$ & $9.8 \pm 4.7$ & $<-1.17$ \\
\hline J0215.1-0422 & 021507.20 & 1.08 & -042212.72 & 1.76 & $S$ & $16.1 \pm 1.9$ & $16.1 \pm 1.9$ & $<3.8$ & $<3.2$ & $63.6 \pm 14.4$ & -0.40 \\
\hline J0215.1-0315 & 021507.30 & 1.09 & -031516.52 & 1.78 & $S$ & $15.2 \pm 2.0$ & $15.2 \pm 2.0$ & $<4.8$ & $<4.0$ & $8.1 \pm 19.2$ & -0.74 \\
\hline J0215.2-0449 & 021511.17 & 1.10 & -044936.50 & 1.81 & $S$ & $9.2 \pm 1.4$ & $9.2 \pm 1.4$ & $<5.8$ & $<4.4$ & $9.2 \pm 16.4$ & -0.25 \\
\hline J0215.2-0343* & 021511.57 & 1.06 & -034311.70 & 1.75 & $M$ & $397.5 \pm 37.1$ & $367.1 \pm 37.0$ & $8.4 \pm 0.4$ & $3.4 \pm 0.3$ & $30.6 \pm 1.1$ & -0.50 \\
\hline & 021511.10 & 1.09 & -034318.96 & 1.83 & $C$ & & $18.2 \pm 2.5$ & $<6.7$ & $<2.5$ & $23.6 \pm 2.5$ & \\
\hline & 021511.33 & 1.12 & -034324.66 & 1.77 & $C$ & & $12.2 \pm 2.0$ & $<5.4$ & $<3.2$ & $57.7 \pm 9.4$ & \\
\hline J0215.4-0343 & 021521.44 & 1.40 & -034322.07 & 1.98 & $S$ & $9.5 \pm 2.2$ & $9.5 \pm 2.2$ & $<10.1$ & $<6.9$ & $43.7 \pm 19.8$ & $<-0.79$ \\
\hline J0215.4-0305 & 021522.01 & 1.09 & -030536.61 & 1.78 & $S$ & $12.0 \pm 1.7$ & $12.0 \pm 1.7$ & $<5.1$ & $<3.8$ & $158.0 \pm 14.0$ & -0.47 \\
\hline J0215.4-0344 & 021522.68 & 1.10 & -034442.86 & 1.79 & $S$ & $16.0 \pm 2.4$ & $16.0 \pm 2.4$ & $<5.2$ & $<4.5$ & $21.2 \pm 34.7$ & -0.79 \\
\hline J0215.4-0416 & 021524.72 & 1.16 & -041618.26 & 1.78 & $S$ & $9.6 \pm 1.4$ & $9.6 \pm 1.4$ & $5.7 \pm 3.8$ & $<4.6$ & $114.4 \pm 14.2$ & -0.41 \\
\hline J0215.4-0435 & 021525.27 & 1.09 & -043558.40 & 1.76 & $S$ & $25.7 \pm 3.0$ & $25.7 \pm 3.0$ & $7.0 \pm 2.0$ & $<2.8$ & $47.7 \pm 3.9$ & -0.70 \\
\hline J0215.5-0320 & 021528.81 & 1.25 & -032015.25 & 1.92 & $S$ & $7.6 \pm 1.7$ & $7.6 \pm 1.7$ & $<8.0$ & $<7.1$ & $-1.0 \pm 71.4$ & $<-0.64$ \\
\hline J0215.5-0532 & 021529.51 & 1.26 & -053258.68 & 1.77 & $M$ & $39.0 \pm 4.2$ & $23.1 \pm 3.5$ & $9.2 \pm 4.1$ & $<4.2$ & $84.0 \pm 6.3$ & -0.22 \\
\hline & 021530.00 & 1.15 & -053256.39 & 1.83 & $C$ & & $8.0 \pm 1.6$ & $<6.3$ & $<5.8$ & $-1.0 \pm 88.6$ & \\
\hline & 021529.10 & 1.19 & -053300.18 & 1.95 & $C$ & & $7.9 \pm 1.7$ & $<8.9$ & $<5.2$ & $25.7 \pm 12.1$ & \\
\hline J0215.5-0418 & 021530.36 & 1.08 & -041811.16 & 1.78 & $M$ & $16.2 \pm 1.8$ & $8.5 \pm 1.3$ & $<4.9$ & $<3.7$ & $171.0 \pm 15.8$ & -1.00 \\
\hline & 021530.17 & 1.14 & -041814.41 & 1.80 & $C$ & & $7.7 \pm 1.3$ & $<6.3$ & $<4.4$ & $43.1 \pm 13.7$ & \\
\hline J0215.5-0319 & 021530.40 & 1.28 & -031902.22 & 1.91 & $S$ & $8.9 \pm 1.9$ & $8.9 \pm 1.9$ & $<7.9$ & $\ldots$ & $\ldots$ & 0.02 \\
\hline J0215.5-0302 & 021531.44 & 1.10 & -030217.21 & 1.78 & $S$ & $14.3 \pm 2.0$ & $14.3 \pm 2.0$ & $<4.7$ & $\ldots$ & $\ldots$ & -1.00 \\
\hline J0215.5-0440 & 021531.57 & 1.23 & -044100.05 & 1.89 & $M$ & $12.0 \pm 1.7$ & $6.1 \pm 1.2$ & $<7.5$ & $<6.9$ & $-1.0 \pm 84.1$ & 0.15 \\
\hline & 021528.31 & 1.38 & -044043.14 & 1.86 & $C$ & & $6.0 \pm 1.2$ & $8.5 \pm 6.1$ & $<6.5$ & $65.5 \pm 19.1$ & \\
\hline J0215.6-0321* & 021535.20 & 1.07 & -032146.00 & 1.75 & $M$ & $314.3 \pm 19.9$ & $133.7 \pm 13.8$ & $10.3 \pm 0.9$ & $3.2 \pm 0.8$ & $42.7 \pm 1.8$ & -0.73 \\
\hline & 021534.66 & 1.10 & -032157.57 & 1.78 & $C$ & & $83.2 \pm 9.8$ & $11.3 \pm 2.0$ & $7.4 \pm 1.6$ & $41.8 \pm 8.4$ & \\
\hline & 021534.36 & 1.31 & -032215.08 & 2.01 & $C$ & & $51.9 \pm 9.0$ & $14.7 \pm 5.4$ & $10.4 \pm 4.1$ & $21.0 \pm 20.6$ & \\
\hline & 021533.57 & 1.09 & -032226.61 & 1.78 & $C$ & & $45.5 \pm 5.4$ & $8.8 \pm 2.0$ & $3.7 \pm 1.9$ & $34.8 \pm 6.4$ & \\
\hline J0215.6-0344 & 021536.14 & 1.18 & -034427.26 & 1.93 & $M$ & $45.0 \pm 4.5$ & $22.8 \pm 3.6$ & $9.9 \pm 5.6$ & $<3.3$ & $32.4 \pm 2.9$ & -0.90 \\
\hline & 021536.37 & 1.08 & -034426.12 & 1.77 & $C$ & & $22.2 \pm 2.8$ & $3.6 \pm 3.1$ & $<3.3$ & $157.9 \pm 9.1$ & \\
\hline J0215.6-0531 & 021536.34 & 1.19 & -053147.15 & 1.76 & $M$ & $31.3 \pm 3.2$ & $20.4 \pm 2.8$ & $7.7 \pm 3.5$ & $<3.5$ & $86.7 \pm 4.9$ & -0.87 \\
\hline & 021536.35 & 1.08 & -053143.76 & 1.77 & $C$ & & $10.9 \pm 1.5$ & $<4.3$ & $<3.6$ & $33.8 \pm 22.3$ & \\
\hline J0215.7-0222* & 021542.03 & 1.06 & -022300.91 & 1.75 & $M$ & $1206.6 \pm 76.0$ & $679.3 \pm 68.1$ & $6.2 \pm 0.2$ & $4.8 \pm 0.2$ & $179.1 \pm 2.7$ & -0.62 \\
\hline & 021541.95 & 1.06 & -022243.52 & 1.75 & $C$ & & $245.7 \pm 25.0$ & $6.0 \pm 0.5$ & $3.8 \pm 0.5$ & $131.0 \pm 4.4$ & \\
\hline & 021541.94 & 1.07 & -022250.73 & 1.75 & $C$ & & $212.4 \pm 21.9$ & $11.4 \pm 1.0$ & $<1.9$ & $78.4 \pm 1.0$ & \\
\hline & 021542.62 & 1.08 & -022258.59 & 1.82 & $C$ & & $39.3 \pm 5.1$ & $6.1 \pm 3.7$ & $<2.6$ & $162.6 \pm 2.7$ & \\
\hline & 021541.70 & 1.06 & -022306.62 & 1.75 & $C$ & & $29.9 \pm 3.4$ & $<2.7$ & $<1.9$ & $164.2 \pm 6.1$ & \\
\hline J0215.7-0401 & 021543.40 & 1.18 & -040114.87 & 2.09 & $S$ & $5.2 \pm 1.1$ & $5.2 \pm 1.1$ & $<10.1$ & $<6.0$ & $9.3 \pm 13.0$ & $<-0.38$ \\
\hline J0215.8-0340 & 021545.11 & 1.09 & -034010.31 & 1.77 & $M$ & $28.9 \pm 3.1$ & $17.4 \pm 2.3$ & $<4.5$ & $<3.4$ & $132.2 \pm 14.1$ & -0.82 \\
\hline & 021544.91 & 1.13 & -034012.81 & 1.80 & $C$ & & $11.5 \pm 2.1$ & $<5.6$ & $\ldots$ & $\ldots$ & \\
\hline J0215.8-0433 & 021545.60 & 1.10 & -043335.32 & 1.83 & $S$ & $4.5 \pm 0.9$ & $4.5 \pm 0.9$ & $<6.4$ & $<4.4$ & $4.0 \pm 18.7$ & $<-0.28$ \\
\hline J0215.8-0335 & 021546.12 & 1.06 & -033554.78 & 1.75 & $M$ & $120.1 \pm 9.2$ & $74.3 \pm 7.7$ & $5.2 \pm 0.9$ & $<2.0$ & $150.4 \pm 3.0$ & -0.36 \\
\hline & 021545.93 & 1.07 & -033556.16 & 1.77 & $C$ & & $45.7 \pm 5.1$ & $7.1 \pm 1.8$ & $<2.4$ & $30.0 \pm 2.4$ & \\
\hline J0215.9-0442 & 021551.18 & 1.07 & -044226.56 & 1.77 & $M$ & $13.3 \pm 1.5$ & $8.1 \pm 1.1$ & $<4.5$ & $<3.3$ & $14.1 \pm 11.2$ & -0.37 \\
\hline & 021551.14 & 1.17 & -044231.22 & 1.82 & $C$ & & $5.2 \pm 1.0$ & $<6.1$ & $\ldots$ & $\ldots$ & \\
\hline J0215.9-0359* & 021552.24 & 1.06 & -035953.04 & 1.75 & $S$ & $109.3 \pm 11.1$ & $109.3 \pm 11.1$ & $6.5 \pm 0.5$ & $3.0 \pm 0.6$ & $104.8 \pm 2.6$ & -0.54 \\
\hline $\mathrm{J} 0216.0-0350 *$ & 021557.95 & 1.07 & -035027.32 & 1.76 & $M$ & $96.2 \pm 7.4$ & $36.4 \pm 4.0$ & $3.6 \pm 1.7$ & $<2.9$ & $22.8 \pm 12.0$ & -0.67 \\
\hline & 021557.85 & 1.47 & -035031.94 & 1.78 & C & & $33.4 \pm 4.9$ & $15.9 \pm 5.3$ & $<4.3$ & $79.8 \pm 3.5$ & \\
\hline & 021557.53 & 1.19 & -035042.60 & 1.80 & $C$ & & $26.4 \pm 3.8$ & $9.5 \pm 3.4$ & $5.1 \pm 2.9$ & $62.7 \pm 12.6$ & \\
\hline
\end{tabular}


Table A.1. continued.

\begin{tabular}{|c|c|c|c|c|c|c|c|c|c|c|c|}
\hline Name & $\begin{array}{c}\text { RA } \\
\text { J2000 }\end{array}$ & $\begin{array}{c}\sigma_{\alpha} \\
\operatorname{arcsec}\end{array}$ & $\begin{array}{c}\text { Dec } \\
\text { J2000 }\end{array}$ & $\begin{array}{c}\sigma_{\delta} \\
\operatorname{arcsec}\end{array}$ & Type & $\begin{array}{l}S_{\text {int }} \\
\text { mJy }\end{array}$ & $\begin{array}{c}S_{\text {comp }} \\
\text { mJy }\end{array}$ & $\begin{array}{c}\text { Major axis } \\
\text { arcsec }\end{array}$ & $\begin{array}{c}\text { Minor axis } \\
\operatorname{arcsec}\end{array}$ & $\begin{array}{c}\text { Position } \\
\text { Angle }\end{array}$ & $\begin{array}{c}\text { Spectral } \\
\text { index } \alpha_{325}^{1400}\end{array}$ \\
\hline J0216.0-0430 & 021558.73 & 1.10 & -043054.77 & 1.76 & $S$ & $9.7 \pm 1.3$ & $9.7 \pm 1.3$ & $<4.4$ & $<3.7$ & $74.3 \pm 20.7$ & -0.74 \\
\hline \multirow[t]{4}{*}{ J0216.0-0344* } & 021600.01 & 1.06 & -034422.84 & 1.75 & $M$ & $384.5 \pm 25.2$ & $205.5 \pm 20.7$ & $5.0 \pm 0.4$ & $<1.4$ & $152.2 \pm 1.8$ & -0.82 \\
\hline & 021559.83 & 1.07 & -034424.44 & 1.76 & $C$ & & $126.6 \pm 13.2$ & $10.1 \pm 1.2$ & $<1.9$ & $30.9 \pm 1.2$ & \\
\hline & 021559.11 & 1.18 & -034416.10 & 1.76 & $C$ & & $33.0 \pm 4.5$ & $6.9 \pm 3.6$ & $<3.1$ & $101.0 \pm 3.8$ & \\
\hline & 021559.11 & 1.16 & -034419.94 & 1.78 & $C$ & & $19.3 \pm 2.9$ & $<5.8$ & $<4.6$ & $92.9 \pm 17.8$ & \\
\hline J0216.0-0432 & 021600.90 & 1.11 & -043245.62 & 1.78 & $S$ & $8.4 \pm 1.2$ & $8.4 \pm 1.2$ & $<5.6$ & $<3.8$ & $44.3 \pm 9.6$ & -0.53 \\
\hline J0216.0-0244 & 021600.98 & 1.44 & -024451.50 & 1.80 & $S$ & $6.5 \pm 1.4$ & $6.5 \pm 1.4$ & $<9.1$ & $<5.5$ & $83.6 \pm 13.3$ & -0.39 \\
\hline \multirow[t]{2}{*}{ J0216.1-0510 } & 021603.26 & 1.08 & -051010.02 & 1.79 & $M$ & $14.2 \pm 1.7$ & $9.0 \pm 1.3$ & $<5.1$ & $<3.6$ & $172.4 \pm 11.0$ & -0.80 \\
\hline & 021603.01 & 1.50 & $\begin{array}{llll}-05 & 10 & 13.73\end{array}$ & 1.84 & $C$ & & $5.2 \pm 1.1$ & $<9.6$ & $<6.6$ & $82.9 \pm 18.8$ & \\
\hline \multirow[t]{2}{*}{ J0216.1-0507 } & 021603.66 & 1.06 & -050759.27 & 1.75 & $M$ & $45.7 \pm 3.6$ & $25.9 \pm 2.7$ & $<2.4$ & $<2.2$ & $163.6 \pm 12.1$ & -0.61 \\
\hline & $\begin{array}{llll}02 & 16 & 03.43\end{array}$ & 1.09 & -050800.76 & 1.78 & $C$ & & $19.8 \pm 2.3$ & $7.7 \pm 2.7$ & $<2.3$ & $38.5 \pm 1.8$ & \\
\hline J0216.1-0256 & 021603.79 & 1.18 & -025649.49 & 1.79 & $S$ & $8.8 \pm 1.5$ & $8.8 \pm 1.5$ & $<6.2$ & $<5.2$ & $96.6 \pm 28.0$ & $<-0.74$ \\
\hline \multirow[t]{3}{*}{ J0216.1-0244 } & 021603.83 & 1.30 & -024351.71 & 1.85 & $M$ & $48.6 \pm 5.1$ & $21.4 \pm 3.4$ & $12.8 \pm 4.8$ & $4.2 \pm 3.7$ & $54.5 \pm 7.7$ & -0.45 \\
\hline & 021604.32 & 1.09 & -024354.89 & 2.08 & $C$ & & $14.9 \pm 2.7$ & $10.4 \pm 6.6$ & $<4.2$ & $1.9 \pm 4.5$ & \\
\hline & 021603.80 & 1.38 & -024403.36 & 1.98 & $C$ & & $12.2 \pm 2.6$ & $9.5 \pm 6.8$ & $<7.3$ & $41.8 \pm 22.2$ & \\
\hline $\mathrm{J} 0216.1-0503$ & 021604.14 & 1.17 & -050313.50 & 1.83 & $S$ & $6.3 \pm 1.2$ & $6.3 \pm 1.2$ & $<6.6$ & $<5.7$ & $144.3 \pm 47.2$ & -0.67 \\
\hline J0216.1-0234 & 021607.32 & 1.23 & -023433.90 & 1.92 & $S$ & $7.4 \pm 1.6$ & $7.4 \pm 1.6$ & $<8.2$ & $<6.7$ & $18.5 \pm 40.1$ & $<-0.62$ \\
\hline \multirow[t]{2}{*}{ J0216.1-0226 } & 021607.49 & 1.08 & -022601.83 & 1.76 & $M$ & $56.2 \pm 5.0$ & $38.3 \pm 4.4$ & $6.1 \pm 1.8$ & $<3.0$ & $50.0 \pm 6.1$ & -0.57 \\
\hline & 021607.89 & 1.09 & -022559.52 & 1.77 & $C$ & & $17.8 \pm 2.3$ & $<4.6$ & $<3.6$ & $143.6 \pm 12.9$ & \\
\hline J0216.1-0448 & 021607.80 & 1.10 & -044845.32 & 2.06 & $S$ & $5.3 \pm 1.0$ & $5.3 \pm 1.0$ & $<9.8$ & $<4.3$ & $176.8 \pm 5.6$ & $<-0.38$ \\
\hline J0216.1-0446 & 021608.36 & 1.07 & -044646.33 & 1.75 & $S$ & $28.8 \pm 3.1$ & $28.8 \pm 3.1$ & $5.0 \pm 1.3$ & $<2.3$ & $59.4 \pm 3.7$ & -0.41 \\
\hline \multirow[t]{2}{*}{ J0216.2-0426 } & 021610.91 & 1.06 & -042608.85 & 1.75 & $M$ & $23.4 \pm 2.2$ & $18.6 \pm 2.0$ & $<2.8$ & $<2.5$ & $145.8 \pm 15.4$ & -0.01 \\
\hline & 021610.62 & 1.20 & -042611.94 & 1.83 & $C$ & & $4.8 \pm 0.9$ & $<7.7$ & $<4.4$ & $46.6 \pm 10.3$ & \\
\hline J0216.2-0321 & 021613.61 & 1.11 & -032138.48 & 1.85 & $S$ & $17.2 \pm 2.6$ & $17.2 \pm 2.6$ & $8.6 \pm 3.7$ & $<4.7$ & $0.9 \pm 10.5$ & -0.32 \\
\hline J0216.3-0512 & 021616.68 & 1.19 & -051257.31 & 1.85 & $S$ & $5.4 \pm 1.0$ & $5.4 \pm 1.0$ & $<7.7$ & $<4.5$ & $42.2 \pm 10.1$ & 0.15 \\
\hline J0216.3-0507 & 021617.93 & 1.14 & -050721.89 & 1.86 & $S$ & $4.1 \pm 0.8$ & $4.1 \pm 0.8$ & $<7.2$ & $<4.9$ & $26.6 \pm 19.2$ & $<-0.21$ \\
\hline J0216.3-0245* & 021620.00 & 1.13 & -024531.98 & 1.96 & $M$ & $314.8 \pm 32.4$ & $278.8 \pm 32.0$ & $34.8 \pm 4.4$ & $12.2 \pm 1.6$ & $164.5 \pm 1.9$ & 0.01 \\
\hline & 021620.96 & 1.21 & -024542.01 & 1.88 & $C$ & & $27.4 \pm 4.7$ & $9.5 \pm 4.7$ & $<5.6$ & $144.6 \pm 13.2$ & \\
\hline & 021619.80 & 1.18 & -024427.73 & 2.23 & $S$ & & $8.7 \pm 2.2$ & $<11.4$ & $<6.1$ & $174.0 \pm 12.7$ & 0.01 \\
\hline J0216.3-0459 & 021620.25 & 1.10 & -045925.00 & 1.79 & $S$ & $5.2 \pm 0.9$ & $5.2 \pm 0.9$ & $<5.2$ & $<4.4$ & $33.3 \pm 33.4$ & $<-0.37$ \\
\hline J0216.3-0532 & 021621.12 & 1.06 & -053258.43 & 1.79 & $M$ & $12.3 \pm 1.5$ & $6.4 \pm 0.7$ & $<5.3$ & $<0.7$ & $0.9 \pm 0.3$ & $<-0.97$ \\
\hline & 021620.79 & 1.53 & -053258.95 & 1.92 & $C$ & & $5.9 \pm 1.4$ & $<10.3$ & $<7.2$ & $64.0 \pm 20.6$ & \\
\hline J0216.5-0345 & 021627.15 & 1.06 & -034519.30 & 1.75 & $S$ & $43.4 \pm 4.5$ & $43.4 \pm 4.5$ & $4.1 \pm 0.9$ & $<2.1$ & $57.3 \pm 5.2$ & -0.90 \\
\hline J0216.5-0242 & 021629.01 & 1.09 & -024235.58 & 1.77 & $S$ & $16.8 \pm 2.1$ & $16.8 \pm 2.1$ & $<4.2$ & $<4.0$ & $58.0 \pm 57.4$ & -1.10 \\
\hline J0216.5-0454 & 021630.87 & 1.14 & -045450.06 & 1.79 & $S$ & $4.4 \pm 0.9$ & $4.4 \pm 0.9$ & $<5.7$ & $<4.9$ & $54.8 \pm 49.4$ & -0.21 \\
\hline J0216.5-0447 & 021632.25 & 1.22 & -044742.40 & 1.86 & $C$ & $4.9 \pm 1.0$ & $4.9 \pm 1.0$ & $<7.4$ & $<6.2$ & $41.1 \pm 42.9$ & \\
\hline J0216.6-0311 & 021634.78 & 1.08 & -031111.64 & 1.78 & $S$ & $12.0 \pm 1.6$ & $12.0 \pm 1.6$ & $<4.6$ & $<3.8$ & $21.1 \pm 19.0$ & 0.82 \\
\hline J0216.6-0454 & 021634.96 & 1.38 & -045429.56 & 1.99 & $S$ & $4.4 \pm 1.0$ & $4.4 \pm 1.0$ & $<10.6$ & $<5.4$ & $42.2 \pm 9.4$ & -0.21 \\
\hline J0216.6-0447 & 021635.56 & 1.08 & -044700.93 & 1.76 & $M$ & $49.3 \pm 3.9$ & $26.6 \pm 3.0$ & $6.1 \pm 1.6$ & $<3.0$ & $56.0 \pm 6.5$ & -0.58 \\
\hline & 021634.75 & 1.15 & -044702.57 & 1.76 & $C$ & & $17.3 \pm 2.3$ & $7.5 \pm 3.0$ & $<3.3$ & $71.6 \pm 4.4$ & \\
\hline & 021634.61 & 1.16 & -044659.35 & 1.78 & $C$ & & $5.4 \pm 1.0$ & $<6.2$ & $<4.3$ & $117.0 \pm 16.8$ & \\
\hline J0216.6-0409 & 021637.07 & 1.20 & -041000.53 & 1.90 & $S$ & $4.9 \pm 1.0$ & $4.9 \pm 1.0$ & $<7.7$ & $<6.5$ & $7.7 \pm 38.6$ & $<-0.34$ \\
\hline J0216.6-0447 & 021637.46 & 1.14 & -044736.55 & 1.77 & $C$ & $8.4 \pm 1.3$ & $8.4 \pm 1.3$ & $<5.6$ & $<3.9$ & $73.0 \pm 12.5$ & \\
\hline J0216.7-0438 & 021638.98 & 1.08 & -043805.76 & 1.86 & $S$ & $4.8 \pm 0.9$ & $4.8 \pm 0.9$ & $<7.1$ & $<3.8$ & $174.6 \pm 9.1$ & $<-0.32$ \\
\hline J0216.7-0444* & 021640.74 & 1.06 & -044407.80 & 1.75 & $S$ & $153.0 \pm 15.3$ & $153.0 \pm 15.3$ & $2.0 \pm 0.3$ & $<1.1$ & $58.8 \pm 6.5$ & -0.37 \\
\hline J0216.7-0436 & 021642.26 & 1.22 & -043603.08 & 1.89 & $S$ & $4.5 \pm 1.0$ & $4.5 \pm 1.0$ & $<7.8$ & $<6.5$ & $154.4 \pm 49.0$ & $<-0.28$ \\
\hline J0216.7-0224 & 021642.43 & 1.31 & -022447.89 & 1.81 & $M$ & $65.1 \pm 6.9$ & $42.0 \pm 6.3$ & $13.2 \pm 4.2$ & $6.8 \pm 2.8$ & $73.8 \pm 9.4$ & -0.75 \\
\hline & $02 \quad 1642.44$ & 1.07 & -022442.76 & 1.76 & $C$ & & $23.1 \pm 2.7$ & $<3.7$ & $<3.3$ & $170.7 \pm 25.7$ & \\
\hline J0216.7-0341 & 021642.62 & 1.07 & -034119.68 & 1.77 & $S$ & $22.6 \pm 2.6$ & $22.6 \pm 2.6$ & $6.4 \pm 1.9$ & $<3.1$ & $15.7 \pm 5.5$ & -0.73 \\
\hline J0216.7-0358 & $02 \quad 1643.59$ & 1.20 & -035817.82 & 1.86 & $S$ & $5.9 \pm 1.2$ & $5.9 \pm 1.2$ & $<7.0$ & $<6.5$ & $-1.0 \pm 87.3$ & 0.27 \\
\hline J0216.7-0306 & 021644.78 & 1.16 & -030617.06 & 1.80 & $S$ & $6.3 \pm 1.1$ & $6.3 \pm 1.1$ & $<6.7$ & $<4.0$ & $50.1 \pm 10.4$ & -0.17 \\
\hline J0216.8-0337* & 021645.78 & 1.08 & -033719.13 & 1.77 & $M$ & $150.7 \pm 11.2$ & $85.5 \pm 9.0$ & $14.9 \pm 1.6$ & $<2.1$ & $35.7 \pm 0.9$ & -0.74 \\
\hline & 021645.78 & 1.06 & -033720.83 & 1.75 & $C$ & & $65.2 \pm 6.7$ & $4.7 \pm 0.8$ & $<1.9$ & $148.9 \pm 3.3$ & \\
\hline J0216.8-0237* & $02 \quad 1647.21$ & 1.06 & -023721.60 & 1.75 & $S$ & $82.1 \pm 8.3$ & $82.1 \pm 8.3$ & $2.0 \pm 0.8$ & $<1.7$ & $1.2 \pm 21.7$ & -0.76 \\
\hline J0216.8-0401 & $02 \quad 1649.10$ & 1.18 & -040144.50 & 1.92 & $M$ & $10.3 \pm 1.4$ & $5.3 \pm 1.0$ & $<8.1$ & $<6.1$ & $166.6 \pm 21.8$ & -0.56 \\
\hline & 021649.32 & 1.15 & -040133.91 & 1.88 & $C$ & & $5.0 \pm 1.0$ & $<7.4$ & $<5.7$ & $176.2 \pm 24.7$ & \\
\hline J0216.8-0427 & 021649.51 & 1.23 & -042739.88 & 1.88 & $S$ & $4.4 \pm 1.0$ & $4.4 \pm 1.0$ & $<7.3$ & $\ldots$ & $\ldots$ & -0.51 \\
\hline J0216.9-0315 & 021650.90 & 1.27 & -031528.94 & 1.85 & $S$ & $5.9 \pm 1.2$ & $5.9 \pm 1.2$ & $<8.0$ & $<5.8$ & $52.7 \pm 21.8$ & 0.23 \\
\hline J0216.9-0215 & 021651.00 & 1.13 & -021527.01 & 1.82 & $S$ & $9.7 \pm 1.8$ & $9.7 \pm 1.8$ & $<6.2$ & $<5.2$ & $158.0 \pm 38.2$ & 0.10 \\
\hline J0216.9-0333 & 021651.55 & 1.34 & -033351.85 & 1.87 & $S$ & $7.0 \pm 1.4$ & $7.0 \pm 1.4$ & $<9.2$ & $<5.0$ & $53.1 \pm 9.2$ & -0.22 \\
\hline J0216.9-0314 & 021653.27 & 1.10 & -031416.44 & 1.77 & $M$ & $85.3 \pm 6.7$ & $37.0 \pm 4.4$ & $7.2 \pm 1.9$ & $5.5 \pm 1.8$ & $93.1 \pm 21.4$ & -0.86 \\
\hline & 021655.15 & 2.22 & -031353.20 & 1.77 & $C$ & & $27.3 \pm 4.5$ & $24.3 \pm 9.6$ & $<4.1$ & $94.7 \pm 2.0$ & \\
\hline & 021654.94 & 1.07 & -031349.23 & 1.76 & $C$ & & $21.0 \pm 2.4$ & $<3.3$ & $<2.9$ & $137.5 \pm 19.1$ & \\
\hline J0216.9-0539 & 021653.36 & 1.07 & -053939.55 & 1.76 & $S$ & $26.8 \pm 3.0$ & $26.8 \pm 3.0$ & $4.1 \pm 1.7$ & $<2.4$ & $42.8 \pm 4.3$ & -0.82 \\
\hline J0217.0-0401 & 021657.00 & 1.12 & -040138.41 & 1.80 & $S$ & $4.0 \pm 0.7$ & $4.0 \pm 0.7$ & $<5.5$ & $<5.0$ & $-1.0 \pm 76.4$ & $<-0.20$ \\
\hline J0217.0-0321 & 021657.26 & 1.26 & -032111.78 & 1.90 & $S$ & $6.0 \pm 1.3$ & $6.0 \pm 1.3$ & $<8.6$ & $<5.8$ & $40.6 \pm 18.6$ & $<-0.48$ \\
\hline J0217.0-0548 & 021657.64 & 1.46 & -054811.81 & 1.80 & $S$ & $5.8 \pm 1.2$ & $5.8 \pm 1.2$ & $<9.3$ & $<5.2$ & $80.5 \pm 11.1$ & $<-0.45$ \\
\hline J0217.0-0220 & 021658.13 & 1.11 & -022056.04 & 1.76 & $S$ & $24.3 \pm 3.0$ & $24.3 \pm 3.0$ & $6.0 \pm 2.5$ & $<3.3$ & $108.9 \pm 6.0$ & -0.74 \\
\hline J0217.0-0308 & 021658.81 & 1.26 & -030839.91 & 1.80 & $M$ & $19.8 \pm 2.6$ & $13.4 \pm 2.3$ & $7.8 \pm 4.5$ & $<5.2$ & $102.7 \pm 13.7$ & -0.93 \\
\hline & 021658.23 & 1.16 & -030837.73 & 1.91 & $C$ & & $6.4 \pm 1.3$ & $<8.0$ & $<5.6$ & $16.4 \pm 17.6$ & \\
\hline J0217.0-0449* & 021658.86 & 1.11 & -045006.23 & 2.58 & $S$ & $96.4 \pm 9.5$ & $71.0 \pm 9.0$ & $48.2 \pm 9.0$ & $7.6 \pm 1.8$ & $4.8 \pm 1.2$ & -1.66 \\
\hline & 021658.98 & 1.13 & -044849.89 & 2.24 & $M$ & & $16.7 \pm 2.8$ & $19.2 \pm 7.1$ & $<5.1$ & $5.2 \pm 4.0$ & -1.66 \\
\hline & 021659.06 & 1.24 & -044830.64 & 2.00 & $C$ & & $8.8 \pm 1.8$ & $9.7 \pm 5.9$ & $<7.1$ & $5.5 \pm 23.9$ & \\
\hline J0217.0-0516 & 021702.60 & 1.10 & -051620.50 & 1.82 & $S$ & $4.6 \pm 0.8$ & $4.6 \pm 0.8$ & $<6.4$ & $<3.5$ & $28.5 \pm 8.5$ & $<-0.29$ \\
\hline
\end{tabular}


Table A.1. continued.

\begin{tabular}{|c|c|c|c|c|c|c|c|c|c|c|c|}
\hline Name & $\begin{array}{c}\text { RA } \\
\text { J2000 }\end{array}$ & $\begin{array}{c}\sigma_{\alpha} \\
\operatorname{arcsec}\end{array}$ & $\begin{array}{c}\text { Dec } \\
\text { J2000 }\end{array}$ & $\begin{array}{c}\sigma_{\delta} \\
\operatorname{arcsec}\end{array}$ & Type & $\begin{array}{l}S_{\text {int }} \\
\text { mJy }\end{array}$ & $\begin{array}{c}S_{\text {comp }} \\
\text { mJy }\end{array}$ & $\begin{array}{c}\text { Major axis } \\
\text { arcsec }\end{array}$ & $\begin{array}{c}\text { Minor axis } \\
\text { arcsec }\end{array}$ & $\begin{array}{c}\text { Position } \\
\text { Angle }\end{array}$ & $\begin{array}{c}\text { Spectral } \\
\text { index } \alpha_{325}^{1400}\end{array}$ \\
\hline \multirow[t]{3}{*}{ J0217.1-0210* } & 021702.80 & 1.06 & -021045.51 & 1.75 & $\bar{M}$ & $266.7 \pm 20.1$ & $180.8 \pm 18.3$ & $6.7 \pm 0.5$ & $4.7 \pm 0.5$ & $44.0 \pm 4.4$ & -0.67 \\
\hline & 021704.30 & 1.07 & -021050.74 & 1.76 & $C$ & & $75.7 \pm 8.0$ & $6.1 \pm 1.0$ & $3.3 \pm 1.1$ & $9.2 \pm 7.0$ & \\
\hline & 021703.35 & 1.12 & -021046.94 & 1.83 & $C$ & & $10.2 \pm 1.9$ & $<6.5$ & $<4.9$ & $12.8 \pm 22.7$ & \\
\hline $\mathrm{J} 0217.1-0430$ & 021703.10 & 1.10 & -043033.01 & 2.17 & $S$ & $4.8 \pm 1.0$ & $4.8 \pm 1.0$ & $<10.9$ & $<4.6$ & $178.0 \pm 6.0$ & $<-0.32$ \\
\hline \multirow[t]{2}{*}{ J0217.0-0421 } & 021703.11 & 1.09 & -042150.47 & 1.83 & $M$ & $11.2 \pm 1.4$ & $6.0 \pm 1.0$ & $<6.6$ & $<3.2$ & $158.5 \pm 5.8$ & $<-0.90$ \\
\hline & 021702.41 & 1.22 & -042132.95 & 1.98 & $C$ & & $5.2 \pm 1.0$ & $<9.6$ & $<4.0$ & $148.2 \pm 5.7$ & \\
\hline $\mathrm{J} 0217.1-0422 *$ & 021705.51 & 1.06 & -042256.85 & 1.75 & $S$ & $153.1 \pm 15.4$ & $153.1 \pm 15.4$ & $2.9 \pm 0.3$ & $1.0 \pm 0.7$ & $43.7 \pm 4.4$ & -0.53 \\
\hline J0217.1-0305 & 021706.36 & 1.08 & -030551.28 & 1.77 & $S$ & $15.5 \pm 1.9$ & $15.5 \pm 1.9$ & $<4.0$ & $\ldots$ & $\ldots$ & -0.30 \\
\hline J0217.1-0315 & 021706.76 & 1.74 & -031512.00 & 1.92 & $S$ & $11.1 \pm 2.5$ & $11.1 \pm 2.5$ & $12.1 \pm 8.2$ & $<7.4$ & $72.3 \pm 14.9$ & -0.63 \\
\hline J0217.1-0337 & 021707.68 & 1.23 & -033725.14 & 1.81 & $S$ & $4.1 \pm 0.9$ & $4.1 \pm 0.9$ & $<6.9$ & $<5.7$ & $82.9 \pm 42.7$ & -0.41 \\
\hline J0217.2-0244 & 021709.42 & 1.12 & -024438.21 & 1.80 & $S$ & $8.9 \pm 1.4$ & $8.9 \pm 1.4$ & $<5.7$ & $<4.5$ & $36.6 \pm 19.2$ & -0.56 \\
\hline J0217.2-0310 & 021712.97 & 1.07 & -031025.24 & 1.76 & $S$ & $16.5 \pm 1.9$ & $16.5 \pm 1.9$ & $<3.7$ & $<3.0$ & $0.2 \pm 11.6$ & -0.57 \\
\hline J0217.2-0353 & 021714.12 & 1.10 & -035343.73 & 1.84 & $S$ & $4.6 \pm 0.8$ & $4.6 \pm 0.8$ & $<6.6$ & $<4.2$ & $13.6 \pm 13.0$ & -0.48 \\
\hline \multirow[t]{2}{*}{ J0217.3-0248 } & 021716.07 & 1.15 & -024804.53 & 1.76 & $M$ & $44.5 \pm 4.1$ & $32.2 \pm 3.8$ & $11.8 \pm 2.5$ & $<2.9$ & $69.5 \pm 2.2$ & -0.92 \\
\hline & 021716.27 & 1.08 & -024800.07 & 1.76 & $C$ & & $12.3 \pm 1.5$ & $<3.8$ & $<3.6$ & $158.3 \pm 50.0$ & \\
\hline J0217.3-0407 & 021716.82 & 1.11 & -040751.57 & 1.77 & $S$ & $4.6 \pm 0.8$ & $4.6 \pm 0.8$ & $<4.8$ & $<4.0$ & $81.1 \pm 30.5$ & -0.54 \\
\hline J0217.3-0333 & 021718.13 & 1.15 & -033339.31 & 1.86 & $S$ & $5.6 \pm 1.2$ & $5.6 \pm 1.2$ & $<7.2$ & $<5.5$ & $22.8 \pm 27.3$ & $<-0.43$ \\
\hline J0217.3-0214 & 021718.41 & 1.18 & -021450.34 & 1.82 & $S$ & $9.7 \pm 1.7$ & $9.7 \pm 1.7$ & $<6.6$ & $<5.6$ & $127.0 \pm 36.2$ & -0.95 \\
\hline J0217.3-0539 & 021718.73 & 1.10 & -053922.10 & 1.78 & $S$ & $6.4 \pm 1.0$ & $6.4 \pm 1.0$ & $<4.9$ & $<4.5$ & $-1.0 \pm 88.5$ & -0.62 \\
\hline \multirow[t]{4}{*}{ J0217.3-0326* } & 021719.84 & 1.06 & -032604.87 & 1.75 & $M$ & $124.1 \pm 10.7$ & $103.8 \pm 10.6$ & $6.9 \pm 0.6$ & $3.7 \pm 0.6$ & $88.6 \pm 3.1$ & -0.97 \\
\hline & 021720.21 & 1.06 & -032606.03 & 1.94 & $C$ & & $7.8 \pm 1.1$ & $<8.2$ & $<1.9$ & $2.6 \pm 1.2$ & \\
\hline & 021719.31 & 1.08 & -032611.18 & 1.80 & $C$ & & $6.7 \pm 1.1$ & $<5.4$ & $<3.8$ & $5.6 \pm 15.3$ & \\
\hline & 021720.41 & 1.11 & -032604.73 & 1.77 & $C$ & & $5.8 \pm 1.0$ & $<5.0$ & $<4.0$ & $61.2 \pm 29.0$ & \\
\hline J0217.3-0318 & 021720.10 & 1.14 & -031855.96 & 2.25 & $S$ & $5.7 \pm 1.2$ & $5.7 \pm 1.2$ & $<11.7$ & $<4.5$ & $167.6 \pm 5.3$ & $<-0.44$ \\
\hline \multirow[t]{2}{*}{ J0217.4-0339* } & 021721.54 & 1.06 & -033948.54 & 1.75 & $M$ & $119.0 \pm 11.4$ & $113.2 \pm 11.4$ & $5.0 \pm 0.3$ & $3.2 \pm 0.4$ & $103.0 \pm 3.5$ & -0.53 \\
\hline & 021721.18 & 1.15 & -033954.38 & 1.81 & $C$ & & $5.8 \pm 1.0$ & $<6.8$ & $<3.6$ & $137.4 \pm 6.9$ & \\
\hline J0217.4-0427 & 021722.23 & 1.07 & -042703.93 & 1.75 & $S$ & $17.6 \pm 1.9$ & $17.6 \pm 1.9$ & $2.5 \pm 2.1$ & $<2.4$ & $41.3 \pm 5.5$ & -0.56 \\
\hline J0217.4-0452 & 021724.78 & 1.15 & -045250.46 & 1.78 & $S$ & $3.9 \pm 0.7$ & $3.9 \pm 0.7$ & $<5.8$ & $<4.7$ & $74.2 \pm 33.5$ & $<-0.18$ \\
\hline J0217.5-0213 & 021727.42 & 1.81 & -021301.75 & 1.82 & $S$ & $19.5 \pm 4.0$ & $19.5 \pm 4.0$ & $13.8 \pm 7.9$ & $<6.0$ & $83.9 \pm 7.7$ & -1.33 \\
\hline J0217.5-0221 & 021730.25 & 1.09 & -022127.20 & 1.76 & $S$ & $31.9 \pm 3.8$ & $31.9 \pm 3.8$ & $6.2 \pm 2.0$ & $<3.5$ & $118.6 \pm 9.8$ & -0.91 \\
\hline \multirow[t]{2}{*}{$\mathrm{J} 0217.5-0224$} & 021730.92 & 1.06 & -022442.57 & 1.75 & $M$ & $108.9 \pm 10.3$ & $100.8 \pm 10.2$ & $1.9 \pm 0.7$ & $<1.5$ & $175.1 \pm 8.2$ & -0.11 \\
\hline & 021730.48 & 1.12 & -022447.67 & 1.77 & $C$ & & $8.1 \pm 1.4$ & $<5.1$ & $<4.4$ & $86.0 \pm 44.2$ & \\
\hline J0217.5-0507 & 021731.04 & 1.13 & -050712.12 & 1.84 & $S$ & $3.7 \pm 0.7$ & $3.7 \pm 0.7$ & $<7.0$ & $<4.4$ & $29.8 \pm 13.2$ & $<-0.15$ \\
\hline J0217.6-0313* & 021734.19 & 1.06 & -031310.48 & 1.75 & $M$ & $103.1 \pm 7.8$ & $64.7 \pm 6.6$ & $4.5 \pm 0.6$ & $<1.6$ & $142.8 \pm 2.2$ & -0.76 \\
\hline & 021734.12 & 1.07 & -031313.05 & 1.75 & $C$ & & $38.4 \pm 4.1$ & $5.7 \pm 1.2$ & $<2.3$ & $57.1 \pm 3.5$ & \\
\hline J0217.6-0408 & 021735.61 & 1.19 & -040806.85 & 2.00 & $S$ & $3.4 \pm 0.7$ & $3.4 \pm 0.7$ & $<9.3$ & $<5.7$ & $19.3 \pm 14.0$ & $<-0.08$ \\
\hline J0217.6-0513 & 021737.78 & 1.44 & -051331.45 & 1.92 & $S$ & $3.9 \pm 0.9$ & $3.9 \pm 0.9$ & $<10.0$ & $<6.2$ & $125.2 \pm 15.4$ & -0.37 \\
\hline J0217.7-0401 & 021740.08 & 1.08 & -040200.75 & 1.76 & $S$ & $10.7 \pm 1.3$ & $10.7 \pm 1.3$ & $<3.8$ & $<3.4$ & $26.5 \pm 25.0$ & $<-0.87$ \\
\hline J0217.7-0541* & 021741.61 & 1.12 & -054155.71 & 1.77 & $M$ & $171.2 \pm 12.3$ & $100.8 \pm 11.0$ & $18.1 \pm 2.0$ & $7.0 \pm 1.0$ & $57.5 \pm 2.1$ & -0.67 \\
\hline & 021740.43 & 1.10 & -054150.63 & 1.77 & $C$ & & $39.5 \pm 4.5$ & $10.8 \pm 2.0$ & $<3.0$ & $49.1 \pm 2.9$ & \\
\hline & 021739.78 & 1.14 & -054153.22 & 1.80 & $C$ & & $16.4 \pm 2.4$ & $7.2 \pm 3.4$ & $<4.6$ & $42.9 \pm 12.1$ & \\
\hline & 021740.77 & 1.22 & -054152.57 & 2.00 & $C$ & & $14.6 \pm 2.5$ & $11.0 \pm 6.4$ & $<4.1$ & $149.4 \pm 4.2$ & \\
\hline J0217.7-0352 & 021742.20 & 1.07 & -035207.53 & 1.76 & $S$ & $23.1 \pm 2.5$ & $23.1 \pm 2.5$ & $4.9 \pm 1.4$ & $<2.6$ & $36.6 \pm 5.5$ & -0.89 \\
\hline J0217.7-0354 & 021742.82 & 1.06 & -035453.55 & 1.75 & $S$ & $29.6 \pm 3.1$ & $29.6 \pm 3.1$ & $2.9 \pm 1.1$ & $<2.3$ & $113.8 \pm 10.8$ & -0.97 \\
\hline J0217.7-0417 & 021743.58 & 1.14 & -041732.47 & 1.77 & $M$ & $8.0 \pm 0.9$ & $4.7 \pm 0.7$ & $<5.9$ & $<2.6$ & $60.0 \pm 3.9$ & -0.76 \\
\hline & 021743.56 & 1.15 & -041729.79 & 1.78 & C & & $3.3 \pm 0.5$ & $<6.3$ & $<2.4$ & $56.6 \pm 3.7$ & \\
\hline J0217.8-0500 & 021745.85 & 1.11 & -050058.87 & 1.78 & $S$ & $4.2 \pm 0.7$ & $4.2 \pm 0.7$ & $<5.2$ & $<4.0$ & $47.1 \pm 25.6$ & $<-0.23$ \\
\hline J0217.8-0239 & 021746.56 & 1.13 & -023909.96 & 1.79 & $S$ & $7.5 \pm 1.3$ & $7.5 \pm 1.3$ & $<6.0$ & $<4.4$ & $45.3 \pm 17.7$ & -0.64 \\
\hline J0217.8-0541 & 021746.87 & 1.13 & -054140.07 & 1.80 & $M$ & $32.8 \pm 3.2$ & $19.6 \pm 2.5$ & $9.5 \pm 3.2$ & $<3.1$ & $42.3 \pm 3.0$ & -0.70 \\
\hline & 021746.93 & 1.15 & -054139.10 & 1.82 & $C$ & & $13.2 \pm 2.0$ & $7.7 \pm 4.2$ & $<3.9$ & $138.6 \pm 5.8$ & \\
\hline J0217.9-0448 & 021752.54 & 1.08 & -044826.17 & 1.76 & $S$ & $10.2 \pm 1.2$ & $10.2 \pm 1.2$ & $<3.6$ & $<3.3$ & $68.4 \pm 33.7$ & -0.54 \\
\hline J0217.9-0512 & 021754.13 & 1.07 & -051251.86 & 1.76 & $S$ & $15.2 \pm 1.7$ & $15.2 \pm 1.7$ & $<3.5$ & $<2.9$ & $64.2 \pm 10.5$ & -0.95 \\
\hline J0217.9-0526 & 021755.09 & 1.10 & -052654.88 & 1.78 & $S$ & $9.5 \pm 1.3$ & $9.5 \pm 1.3$ & $4.5 \pm 3.3$ & $<3.9$ & $40.2 \pm 11.3$ & -0.05 \\
\hline J0218.0-0346 & 021757.07 & 1.21 & -034603.75 & 1.80 & $M$ & $11.0 \pm 1.7$ & $11.0 \pm 1.7$ & $8.4 \pm 3.6$ & $5.6 \pm 3.3$ & $76.3 \pm 23.7$ & -0.18 \\
\hline J0218.0-0346 & 021757.45 & 1.10 & -034648.80 & 1.79 & $C$ & $13.5 \pm 1.4$ & $7.1 \pm 1.0$ & $<5.8$ & $<2.9$ & $36.3 \pm 4.4$ & \\
\hline & 021757.61 & 1.10 & -034649.20 & 1.77 & $C$ & & $6.5 \pm 0.9$ & $<5.2$ & $<3.4$ & $135.1 \pm 9.6$ & \\
\hline J0218.0-0347 & 021758.03 & 1.15 & -034759.76 & 1.85 & $S$ & $3.8 \pm 0.8$ & $3.8 \pm 0.8$ & $<7.3$ & $<4.6$ & $31.2 \pm 13.6$ & $<-0.17$ \\
\hline J0218.0-0432 & 021758.90 & 1.10 & -043257.88 & 1.81 & $S$ & $4.9 \pm 0.8$ & $4.9 \pm 0.8$ & $<5.8$ & $<4.3$ & $9.5 \pm 18.2$ & $<-0.34$ \\
\hline J0218.0-0450 & 021800.65 & 1.15 & -045005.99 & 1.77 & $S$ & $3.9 \pm 0.7$ & $3.9 \pm 0.7$ & $<5.8$ & $<4.0$ & $110.2 \pm 16.2$ & 0.10 \\
\hline J0218.0-0344 & 021801.32 & 1.11 & -034402.13 & 1.78 & $S$ & $11.4 \pm 1.5$ & $11.4 \pm 1.5$ & $5.8 \pm 2.8$ & $<4.2$ & $123.9 \pm 17.0$ & -0.80 \\
\hline J0218.0-0201 & 021801.35 & 1.14 & -020117.74 & 1.79 & $S$ & $12.3 \pm 2.1$ & $12.3 \pm 2.1$ & $<5.7$ & $<4.9$ & $119.0 \pm 35.7$ & $<-0.96$ \\
\hline J0218.0-0341 & 021802.26 & 1.06 & -034131.65 & 1.75 & $S$ & $21.5 \pm 2.3$ & $21.5 \pm 2.3$ & $2.6 \pm 1.5$ & $<2.3$ & $21.6 \pm 7.1$ & -0.82 \\
\hline J0218.1-0306 & 021803.26 & 1.12 & -030625.38 & 1.79 & $S$ & $7.8 \pm 1.2$ & $7.8 \pm 1.2$ & $<5.3$ & $<4.7$ & $49.3 \pm 42.5$ & -0.64 \\
\hline J0218.1-0538 & 021803.28 & 1.11 & -053827.71 & 1.78 & $M$ & $27.0 \pm 2.6$ & $19.3 \pm 2.4$ & $7.8 \pm 2.8$ & $<2.8$ & $47.9 \pm 2.8$ & -0.66 \\
\hline & 021803.40 & 1.14 & -053828.14 & 1.77 & $C$ & & $7.7 \pm 1.2$ & $<5.7$ & $<3.5$ & $117.3 \pm 7.9$ & \\
\hline J0218.1-0456 & 021805.14 & 1.16 & -045625.71 & 1.78 & $S$ & $5.2 \pm 0.9$ & $5.2 \pm 0.9$ & $<6.1$ & $<4.3$ & $118.8 \pm 14.4$ & $<-0.38$ \\
\hline J0218.1-0501 & 021807.47 & 1.10 & -050149.38 & 1.94 & $S$ & $4.3 \pm 0.9$ & $4.3 \pm 0.9$ & $<8.3$ & $<4.4$ & $2.1 \pm 9.4$ & $<-0.25$ \\
\hline J0218.1-0324 & 021807.64 & 1.09 & -032438.95 & 1.77 & $S$ & $8.4 \pm 1.1$ & $8.4 \pm 1.1$ & $<4.4$ & $<3.8$ & $50.7 \pm 24.6$ & -0.61 \\
\hline J0218.1-0546 & 021807.90 & 1.09 & -054605.60 & 1.77 & $S$ & $14.0 \pm 1.8$ & $14.0 \pm 1.8$ & $4.7 \pm 2.5$ & $3.8 \pm 2.7$ & $-1.0 \pm 71.2$ & -0.21 \\
\hline J0218.1-0304 & 021808.31 & 1.17 & -030444.86 & 1.79 & $S$ & $8.2 \pm 1.3$ & $8.2 \pm 1.3$ & $5.0 \pm 4.4$ & $<5.0$ & $66.2 \pm 21.7$ & $<-0.69$ \\
\hline J0218.1-0548 & 021808.81 & 1.14 & -054826.98 & 1.81 & $S$ & $5.5 \pm 1.0$ & $5.5 \pm 1.0$ & $<6.5$ & $<4.2$ & $40.3 \pm 13.9$ & -0.69 \\
\hline J0218.2-0459 & 021809.37 & 1.10 & -045948.25 & 1.80 & $S$ & $6.2 \pm 1.0$ & $6.2 \pm 1.0$ & $<5.4$ & $<4.5$ & $4.2 \pm 29.7$ & $<-0.50$ \\
\hline J0218.2-0319 & 021811.26 & 1.08 & -031937.65 & 1.78 & $M$ & $38.6 \pm 3.0$ & $17.4 \pm 2.2$ & $5.5 \pm 2.3$ & $<3.7$ & $15.0 \pm 12.0$ & -0.77 \\
\hline & 021812.37 & 1.10 & -032023.86 & 1.78 & $C$ & & $10.7 \pm 1.5$ & $<4.6$ & $\ldots$ & $\ldots$ & \\
\hline
\end{tabular}


Table A.1. continued.

\begin{tabular}{|c|c|c|c|c|c|c|c|c|c|c|c|}
\hline Name & $\begin{array}{c}\text { RA } \\
\text { J2000 }\end{array}$ & $\begin{array}{c}\sigma_{\alpha} \\
\operatorname{arcsec}\end{array}$ & $\begin{array}{c}\text { Dec } \\
\text { J2000 }\end{array}$ & $\begin{array}{c}\sigma_{\delta} \\
\operatorname{arcsec}\end{array}$ & Type & $\begin{array}{l}S_{\text {int }} \\
\text { mJy }\end{array}$ & $\begin{array}{c}S_{\text {comp }} \\
\text { mJy }\end{array}$ & $\begin{array}{c}\text { Major axis } \\
\text { arcsec }\end{array}$ & $\begin{array}{c}\text { Minor axis } \\
\text { arcsec }\end{array}$ & $\begin{array}{c}\text { Position } \\
\text { Angle }\end{array}$ & $\begin{array}{c}\text { Spectral } \\
\text { index } \alpha_{325}^{1400}\end{array}$ \\
\hline & 021810.97 & 1.29 & -031947.94 & 1.92 & $C$ & & $5.5 \pm 1.1$ & $<8.9$ & $<6.3$ & $139.4 \pm 18.5$ & \\
\hline & 021812.61 & 1.18 & -032025.34 & 1.86 & $C$ & & $5.0 \pm 1.0$ & $<7.8$ & $<4.6$ & $36.8 \pm 12.1$ & \\
\hline \multirow[t]{3}{*}{ J0218.2-0301* } & 021812.33 & 1.06 & -030132.18 & 1.75 & $M$ & $131.7 \pm 9.5$ & $77.9 \pm 7.9$ & $4.6 \pm 0.6$ & $3.5 \pm 0.6$ & $90.8 \pm 11.2$ & -0.98 \\
\hline & 021811.25 & 1.07 & -030122.45 & 1.76 & $C$ & & $47.9 \pm 5.1$ & $6.2 \pm 1.1$ & $4.6 \pm 1.1$ & $130.9 \pm 12.9$ & \\
\hline & 021811.90 & 1.22 & -030138.16 & 1.86 & $C$ & & $5.9 \pm 1.1$ & $<8.3$ & $<3.9$ & $137.6 \pm 6.6$ & \\
\hline J0218.2-0427 & 021813.51 & 1.20 & -042704.58 & 1.78 & $S$ & $4.6 \pm 0.8$ & $4.6 \pm 0.8$ & $<6.7$ & $<4.7$ & $75.0 \pm 14.9$ & $<-0.30$ \\
\hline J0218.3-0453 & 021815.89 & 1.10 & -045348.92 & 1.88 & $S$ & $4.6 \pm 0.9$ & $4.6 \pm 0.9$ & $<7.4$ & $<4.5$ & $175.8 \pm 12.3$ & $<-0.30$ \\
\hline J0218.3-0316 & 021816.82 & 1.19 & $\begin{array}{llll}-03 & 16 & 03.47\end{array}$ & 2.08 & $S$ & $4.3 \pm 1.0$ & $4.3 \pm 1.0$ & $<10.1$ & $<5.9$ & $15.3 \pm 12.4$ & $<-0.25$ \\
\hline \multirow[t]{3}{*}{ J0218.3-0446* } & 021818.05 & 1.06 & -044609.15 & 1.75 & $M$ & $191.9 \pm 18.1$ & $180.6 \pm 18.1$ & $4.0 \pm 0.2$ & $1.4 \pm 0.4$ & $1.4 \pm 1.8$ & -0.76 \\
\hline & 021817.67 & 1.17 & -044614.61 & 1.89 & $C$ & & $5.9 \pm 0.9$ & $<8.4$ & $<2.2$ & $144.7 \pm 1.6$ & \\
\hline & 021817.70 & 1.08 & -044611.65 & 1.80 & $C$ & & $5.4 \pm 0.7$ & $<5.9$ & $<1.5$ & $153.2 \pm 1.3$ & \\
\hline J0218.3-0515 & 021818.33 & 1.21 & -051547.76 & 1.82 & $S$ & $5.1 \pm 1.0$ & $5.1 \pm 1.0$ & $<7.0$ & $<5.4$ & $122.6 \pm 28.6$ & $<-0.36$ \\
\hline J0218.3-0547 & 021818.72 & 1.12 & -054712.23 & 1.86 & $S$ & $4.9 \pm 1.0$ & $4.9 \pm 1.0$ & $<7.0$ & $<4.9$ & $12.7 \pm 20.3$ & -0.55 \\
\hline \multirow[t]{3}{*}{ J0218.3-0403 } & 021818.85 & 1.13 & -040329.05 & 1.77 & $M$ & $25.5 \pm 2.6$ & $13.3 \pm 1.7$ & $7.4 \pm 2.8$ & $<3.8$ & $67.4 \pm 7.2$ & -0.55 \\
\hline & 021817.75 & 1.62 & -040332.82 & 1.97 & $C$ & & $8.2 \pm 1.8$ & $12.2 \pm 7.9$ & $<6.6$ & $57.5 \pm 10.8$ & \\
\hline & 021820.00 & 1.42 & -040330.57 & 1.86 & $C$ & & $4.1 \pm 0.9$ & $<9.0$ & $<6.8$ & $76.9 \pm 24.8$ & \\
\hline J0218.3-0441 & 021820.34 & 1.08 & -044138.81 & 1.99 & $S$ & $5.9 \pm 1.0$ & $5.9 \pm 1.0$ & $<9.0$ & $<3.3$ & $6.2 \pm 3.8$ & $<-0.47$ \\
\hline J0218.3-0438 & 021820.71 & 1.07 & -043834.97 & 1.98 & $S$ & $5.2 \pm 0.9$ & $5.2 \pm 0.9$ & $<8.8$ & $<3.3$ & $176.4 \pm 4.1$ & $<-0.38$ \\
\hline J0218.4-0516 & 021822.44 & 1.11 & -051651.13 & 1.78 & $S$ & $5.4 \pm 0.9$ & $5.4 \pm 0.9$ & $<5.1$ & $<4.1$ & $51.0 \pm 26.8$ & -0.58 \\
\hline J0218.4-0214 & 021822.58 & 1.10 & -021437.72 & 1.78 & $S$ & $12.4 \pm 1.8$ & $12.4 \pm 1.8$ & $<5.4$ & $<3.5$ & $144.5 \pm 8.0$ & $<-0.97$ \\
\hline \multirow{2}{*}{$\mathrm{J} 0218.4-0542$} & 021822.70 & 1.28 & -054217.07 & 1.77 & $M$ & $32.8 \pm 3.2$ & $20.4 \pm 2.8$ & $12.2 \pm 4.0$ & $<3.7$ & $98.6 \pm 3.5$ & -0.32 \\
\hline & 021822.45 & 1.07 & -054213.45 & 1.76 & $C$ & & $12.4 \pm 1.5$ & $<3.9$ & $<2.7$ & $14.0 \pm 6.8$ & \\
\hline J0218.4-0229 & 021822.71 & 1.08 & -022937.65 & 1.76 & $S$ & $14.7 \pm 1.8$ & $14.7 \pm 1.8$ & $<3.9$ & $<3.4$ & $118.4 \pm 18.9$ & -0.51 \\
\hline J0218.4-0525 & 021823.46 & 1.15 & -052502.50 & 1.89 & $S$ & $5.7 \pm 1.0$ & $5.7 \pm 1.0$ & $<8.1$ & $<3.9$ & $29.7 \pm 6.4$ & 0.49 \\
\hline J0218.4-0419 & 021823.51 & 1.07 & -041902.22 & 1.75 & $S$ & $17.0 \pm 1.8$ & $17.0 \pm 1.8$ & $<2.9$ & $<2.5$ & $47.7 \pm 13.1$ & -0.67 \\
\hline J0218.4-0406 & 021823.59 & 1.20 & -040615.35 & 1.76 & $S$ & $5.1 \pm 0.7$ & $5.1 \pm 0.7$ & $<6.6$ & $<2.7$ & $100.9 \pm 3.3$ & $<-0.36$ \\
\hline J0218.4-0453 & 021824.00 & 1.07 & -045306.51 & 1.76 & $S$ & $17.5 \pm 2.0$ & $17.5 \pm 2.0$ & $<3.3$ & $<2.8$ & $29.0 \pm 11.7$ & -0.52 \\
\hline J0218.4-0543 & 021825.30 & 1.10 & -054359.50 & 1.78 & $S$ & $6.1 \pm 1.0$ & $6.1 \pm 1.0$ & $<5.0$ & $<4.2$ & $41.5 \pm 34.6$ & -0.48 \\
\hline J0218.4-0459 & 021825.36 & 1.12 & -045950.24 & 1.77 & $S$ & $16.9 \pm 2.2$ & $16.9 \pm 2.2$ & $6.4 \pm 2.8$ & $<3.9$ & $54.6 \pm 9.3$ & -1.06 \\
\hline J0218.5-0412 & 021827.22 & 1.21 & -041221.90 & 1.86 & $S$ & $3.9 \pm 0.8$ & $3.9 \pm 0.8$ & $<7.5$ & $<6.2$ & $36.2 \pm 37.8$ & 0.12 \\
\hline \multirow[t]{5}{*}{$\mathrm{J} 0218.5-0454 *$} & 021827.29 & 1.06 & -045439.68 & 1.75 & $M$ & $296.4 \pm 19.5$ & $183.5 \pm 18.4$ & $7.6 \pm 0.3$ & $2.5 \pm 0.4$ & $28.7 \pm 1.0$ & -0.87 \\
\hline & 021827.01 & 1.06 & -045449.32 & 1.75 & $C$ & & $40.5 \pm 4.2$ & $<2.3$ & $<2.0$ & $138.6 \pm 6.7$ & \\
\hline & 021826.86 & 1.07 & -045454.67 & 1.75 & $C$ & & $28.1 \pm 3.0$ & $2.4 \pm 1.7$ & $\ldots$ & $\ldots$ & \\
\hline & 021827.63 & 1.08 & -045428.49 & 1.75 & $C$ & & $25.3 \pm 2.8$ & $4.1 \pm 1.9$ & $<2.1$ & $60.8 \pm 2.6$ & \\
\hline & 021827.56 & 1.08 & -045432.04 & 1.75 & $C$ & & $19.0 \pm 2.1$ & $3.3 \pm 2.5$ & $<1.9$ & $86.4 \pm 2.0$ & \\
\hline J0218.5-0534 & 021827.54 & 1.08 & -053455.78 & 1.76 & $S$ & $13.5 \pm 1.6$ & $13.5 \pm 1.6$ & $3.3 \pm 2.8$ & $<3.4$ & $45.9 \pm 12.8$ & -0.81 \\
\hline \multirow[t]{2}{*}{ J0218.5-0418 } & 021832.62 & 1.13 & -041828.48 & 1.78 & $M$ & $9.4 \pm 1.0$ & $5.3 \pm 0.8$ & $<6.0$ & $<3.2$ & $52.5 \pm 5.8$ & -1.02 \\
\hline & 021832.56 & 1.07 & -041825.68 & 1.77 & $C$ & & $4.0 \pm 0.6$ & $<4.3$ & $<3.0$ & $29.7 \pm 13.3$ & \\
\hline J0218.5-0216 & 021832.79 & 1.24 & -021624.15 & 1.92 & $S$ & $7.3 \pm 1.5$ & $7.3 \pm 1.5$ & $<8.7$ & $<5.5$ & $143.8 \pm 14.0$ & -0.17 \\
\hline J0218.6-0259 & 021833.00 & 1.07 & -025913.98 & 1.76 & $S$ & $18.5 \pm 2.1$ & $18.5 \pm 2.1$ & $<3.6$ & $<3.3$ & $12.8 \pm 31.4$ & -1.03 \\
\hline J0218.5-0313 & 021833.24 & 1.09 & -031333.34 & 1.77 & $M$ & $33.8 \pm 2.5$ & $11.6 \pm 1.5$ & $<4.6$ & $<3.1$ & $133.2 \pm 7.0$ & -0.99 \\
\hline & 021832.99 & 1.11 & -031335.30 & 1.77 & $C$ & & $8.9 \pm 1.3$ & $<5.2$ & $<3.8$ & $54.2 \pm 11.9$ & \\
\hline & 021831.18 & 1.16 & -031343.58 & 1.82 & $C$ & & $7.0 \pm 1.2$ & $<6.8$ & $<4.7$ & $41.6 \pm 13.0$ & \\
\hline & 021832.48 & 1.16 & -031336.01 & 1.79 & $C$ & & $6.2 \pm 1.1$ & $<5.9$ & $<5.2$ & $95.0 \pm 53.4$ & \\
\hline J0218.6-0248 & 021833.67 & 1.07 & -024808.93 & 1.76 & $S$ & $14.4 \pm 1.7$ & $14.4 \pm 1.7$ & $<3.7$ & $<3.4$ & $161.7 \pm 30.5$ & -0.44 \\
\hline J0218.6-0346 & 021834.90 & 1.26 & -034617.10 & 1.82 & $S$ & $4.7 \pm 0.9$ & $4.7 \pm 0.9$ & $<7.6$ & $<5.9$ & $66.4 \pm 25.4$ & $<-0.31$ \\
\hline J0218.6-0246 & 021835.39 & 1.13 & -024627.52 & 1.80 & $S$ & $9.4 \pm 1.5$ & $9.4 \pm 1.5$ & $<6.0$ & $<4.6$ & $41.1 \pm 16.7$ & -0.49 \\
\hline J0218.6-0357 & 021836.88 & 1.21 & -035712.27 & 1.86 & $M$ & $16.5 \pm 1.7$ & $9.0 \pm 1.4$ & $8.3 \pm 5.4$ & $<3.3$ & $42.8 \pm 3.2$ & -1.22 \\
\hline & 021837.00 & 1.12 & -035712.31 & 1.76 & $C$ & & $7.5 \pm 1.0$ & $<5.1$ & $<3.4$ & $113.8 \pm 8.0$ & \\
\hline J0218.6-0334 & 021837.58 & 1.09 & -033412.79 & 1.77 & $S$ & $9.3 \pm 1.2$ & $9.3 \pm 1.2$ & $3.7 \pm 3.1$ & $<4.1$ & $34.1 \pm 33.0$ & -0.66 \\
\hline J0218.6-0541 & 021837.77 & 1.06 & -054200.99 & 1.79 & $S$ & $6.1 \pm 0.6$ & $6.1 \pm 0.6$ & $<5.3$ & $<0.2$ & $173.7 \pm 0.0$ & $<-0.49$ \\
\hline J0218.6-0545 & 021837.80 & 1.06 & -054548.03 & 1.77 & $S$ & $5.4 \pm 0.7$ & $5.4 \pm 0.7$ & $<4.4$ & $<1.5$ & $179.8 \pm 1.9$ & $<-0.40$ \\
\hline J0218.6-0245 & 021837.96 & 1.11 & -024517.67 & 1.88 & $S$ & $8.6 \pm 1.4$ & $8.6 \pm 1.4$ & $6.5 \pm 5.2$ & $<4.2$ & $19.8 \pm 7.0$ & -0.38 \\
\hline J0218.6-0347 & 021838.71 & 1.08 & -034706.77 & 1.77 & $S$ & $6.4 \pm 0.9$ & $6.4 \pm 0.9$ & $<4.2$ & $\ldots$ & $\ldots$ & $<-0.52$ \\
\hline J0218.7-0441* & 021839.37 & 1.06 & -044150.62 & 1.75 & $M$ & $282.4 \pm 24.0$ & $236.7 \pm 23.7$ & $9.5 \pm 0.3$ & $<1.1$ & $112.7 \pm 0.4$ & -1.15 \\
\hline & 021839.88 & 1.06 & -044153.49 & 1.75 & $C$ & & $30.4 \pm 3.1$ & $<2.7$ & $<1.2$ & $20.1 \pm 0.9$ & \\
\hline & 021840.17 & 1.06 & -044156.22 & 1.75 & $C$ & & $15.3 \pm 1.5$ & $<2.6$ & $<0.0$ & $129.1 \pm 0.0$ & \\
\hline J0218.7-0322 & 021840.24 & 1.09 & -032309.63 & 2.15 & $M$ & $30.1 \pm 3.4$ & $18.5 \pm 3.0$ & $16.0 \pm 6.5$ & $<4.3$ & $177.4 \pm 3.4$ & -0.09 \\
\hline & 021840.13 & 1.25 & -032301.41 & 1.80 & $C$ & & $6.4 \pm 1.2$ & $<7.3$ & $<5.5$ & $102.8 \pm 20.9$ & \\
\hline & 021840.34 & 1.42 & -03 2313.77 & 1.81 & $C$ & & $5.2 \pm 1.1$ & $<9.2$ & $<4.9$ & $70.6 \pm 9.3$ & \\
\hline J0218.8-0423 & 021846.65 & 1.17 & -042341.87 & 1.99 & $S$ & $3.4 \pm 0.8$ & $3.4 \pm 0.8$ & $<9.1$ & $<5.7$ & $15.5 \pm 15.4$ & $<-0.10$ \\
\hline J0218.8-0209 & 021846.76 & 1.31 & -020949.92 & 1.84 & $S$ & $9.9 \pm 1.9$ & $9.9 \pm 1.9$ & $<8.7$ & $<4.3$ & $55.1 \pm 7.2$ & -0.12 \\
\hline J0218.8-0414 & 021846.95 & 1.09 & -041419.12 & 1.82 & $M$ & $11.1 \pm 1.3$ & $6.3 \pm 1.0$ & $<6.2$ & $<4.0$ & $13.3 \pm 9.2$ & -0.11 \\
\hline & 021847.51 & 1.30 & -041410.88 & 1.85 & $C$ & & $4.8 \pm 0.9$ & $7.4 \pm 5.7$ & $<6.1$ & $119.3 \pm 19.5$ & \\
\hline J0218.8-0241 & 021847.23 & 1.38 & -024129.23 & 1.88 & $S$ & $6.2 \pm 1.4$ & $6.2 \pm 1.4$ & $<8.6$ & $<7.2$ & $99.1 \pm 45.6$ & 0.28 \\
\hline J0218.8-0458 & 021850.48 & 1.06 & -045833.74 & 1.75 & $S$ & $23.0 \pm 2.4$ & $23.0 \pm 2.4$ & $<2.4$ & $<2.2$ & $10.5 \pm 10.7$ & -0.77 \\
\hline J0218.8-0211* & 021851.04 & 1.06 & -021113.10 & 1.75 & $M$ & $696.9 \pm 49.5$ & $364.9 \pm 36.6$ & $3.5 \pm 0.2$ & $2.5 \pm 0.3$ & $0.1 \pm 4.4$ & -0.72 \\
\hline & 021850.73 & 1.06 & -021122.03 & 1.75 & $C$ & & $332.0 \pm 33.3$ & $5.4 \pm 0.2$ & $1.7 \pm 0.4$ & $177.8 \pm 1.3$ & \\
\hline J0218.9-0509 & 021851.29 & 1.06 & -050904.05 & 1.75 & $S$ & $53.9 \pm 5.4$ & $53.9 \pm 5.4$ & $<1.6$ & $<1.6$ & $25.6 \pm 12.0$ & -0.75 \\
\hline J0218.9-0310 & 021852.07 & 1.17 & -031048.42 & 1.85 & $S$ & $5.3 \pm 1.1$ & $5.3 \pm 1.1$ & $<6.8$ & $<6.1$ & $-1.0 \pm 68.1$ & -0.33 \\
\hline J0218.9-0447 & 021853.54 & 1.06 & -044738.14 & 1.75 & $S$ & $21.5 \pm 2.3$ & $21.5 \pm 2.3$ & $<2.5$ & $<2.2$ & $30.8 \pm 12.1$ & -0.26 \\
\hline J0218.9-0257 & 021855.39 & 1.07 & -025749.29 & 1.76 & $S$ & $44.6 \pm 4.8$ & $44.6 \pm 4.8$ & $4.6 \pm 1.3$ & $<2.7$ & $39.6 \pm 10.3$ & -0.95 \\
\hline J0218.9-0401 & 021856.04 & 1.47 & -040128.28 & 1.83 & $S$ & $10.5 \pm 1.9$ & $10.5 \pm 1.9$ & $11.4 \pm 6.1$ & $<5.1$ & $68.3 \pm 6.8$ & -0.77 \\
\hline J0219.0-0409 & 021858.14 & 1.06 & -040924.85 & 1.75 & $S$ & $40.2 \pm 4.1$ & $40.2 \pm 4.1$ & $2.7 \pm 0.9$ & $<1.7$ & $33.5 \pm 3.8$ & -0.57 \\
\hline
\end{tabular}


C. Tasse et al.: The low-frequency counterpart of the XMM large scale structure survey, Online Material p 10

Table A.1. continued.

\begin{tabular}{|c|c|c|c|c|c|c|c|c|c|c|c|}
\hline Name & $\begin{array}{c}\text { RA } \\
\text { J2000 }\end{array}$ & $\begin{array}{c}\sigma_{\alpha} \\
\operatorname{arcsec}\end{array}$ & $\begin{array}{c}\text { Dec } \\
\text { J2000 }\end{array}$ & $\begin{array}{c}\sigma_{\delta} \\
\operatorname{arcsec}\end{array}$ & Type & $\begin{array}{l}S_{\text {int }} \\
\mathrm{mJy}\end{array}$ & $\begin{array}{c}S_{\text {comp }} \\
\text { mJy }\end{array}$ & $\begin{array}{c}\text { Major axis } \\
\text { arcsec }\end{array}$ & $\begin{array}{c}\text { Minor axis } \\
\text { arcsec }\end{array}$ & $\begin{array}{c}\text { Position } \\
\text { Angle }\end{array}$ & $\begin{array}{c}\text { Spectral } \\
\text { index } \alpha_{325}^{1400}\end{array}$ \\
\hline J0219.0-0225 & 021858.82 & 1.06 & -022546.96 & 1.75 & $\bar{S}$ & $116.9 \pm 11.8$ & $116.9 \pm 11.8$ & $<1.5$ & $<1.4$ & $100.1 \pm 31.0$ & -0.09 \\
\hline J0219.0-0402 & 021859.79 & 1.19 & -040238.98 & 1.85 & $S$ & $4.5 \pm 0.9$ & $4.5 \pm 0.9$ & $<7.6$ & $<5.0$ & $41.7 \pm 15.8$ & $<-0.28$ \\
\hline J0219.0-0355 & 021900.60 & 1.06 & -035559.09 & 1.75 & $S$ & $32.9 \pm 3.4$ & $32.9 \pm 3.4$ & $3.2 \pm 0.9$ & $<2.1$ & $73.5 \pm 8.2$ & -0.58 \\
\hline J0219.1-0305 & 021904.89 & 1.10 & -030508.87 & 1.91 & $S$ & $6.7 \pm 1.4$ & $6.7 \pm 1.4$ & $<7.9$ & $<4.5$ & $7.2 \pm 11.9$ & $<-0.55$ \\
\hline J0219.1-0435 & 021905.48 & 1.08 & -043532.15 & 1.76 & $S$ & $9.6 \pm 1.2$ & $9.6 \pm 1.2$ & $<3.9$ & $<3.0$ & $42.6 \pm 11.0$ & -0.72 \\
\hline J0219.1-0414 & 021905.79 & 1.15 & -041418.76 & 1.89 & $S$ & $4.1 \pm 0.8$ & $4.1 \pm 0.8$ & $<7.6$ & $<5.6$ & $179.1 \pm 22.5$ & -0.34 \\
\hline J0219.1-0459 & 021906.64 & 1.11 & -045904.13 & 1.81 & $S$ & $4.0 \pm 0.7$ & $4.0 \pm 0.7$ & $<5.8$ & $<4.9$ & $4.3 \pm 41.7$ & -0.07 \\
\hline \multirow[t]{3}{*}{ J0219.1-0252* } & 021907.45 & 1.06 & -025253.00 & 1.75 & $M$ & $638.1 \pm 59.5$ & $592.6 \pm 59.3$ & $2.9 \pm 0.2$ & $1.1 \pm 0.4$ & $177.2 \pm 2.5$ & -0.63 \\
\hline & 021907.06 & 1.07 & -025301.64 & 1.78 & $C$ & & $25.6 \pm 3.3$ & $<4.9$ & $<3.1$ & $164.5 \pm 6.6$ & \\
\hline & 021907.16 & 1.08 & -025258.58 & 1.76 & $C$ & & $19.9 \pm 2.6$ & $<4.4$ & $<2.4$ & $133.8 \pm 4.8$ & \\
\hline J0219.1-0516 & 021908.36 & 1.09 & -051637.20 & 1.83 & $S$ & $5.3 \pm 0.9$ & $5.3 \pm 0.9$ & $<6.5$ & $<3.8$ & $12.8 \pm 8.8$ & $<-0.39$ \\
\hline J0219.1-0455 & 021908.45 & 1.15 & -045538.56 & 1.86 & $S$ & $3.5 \pm 0.8$ & $3.5 \pm 0.8$ & $<7.2$ & $<5.2$ & $23.5 \pm 24.0$ & $<-0.11$ \\
\hline \multirow[t]{4}{*}{ J0219.1-0357* } & 021908.77 & 1.06 & -035803.24 & 1.75 & $M$ & $118.2 \pm 8.7$ & $78.9 \pm 8.0$ & $6.1 \pm 0.5$ & $3.5 \pm 0.5$ & $130.6 \pm 3.6$ & -0.63 \\
\hline & 021906.75 & 1.08 & -035743.36 & 1.76 & $C$ & & $27.1 \pm 3.1$ & $5.2 \pm 1.5$ & $4.5 \pm 1.5$ & $91.2 \pm 46.1$ & \\
\hline & 021908.18 & 1.16 & -035755.63 & 1.78 & $C$ & & $6.6 \pm 1.1$ & $<6.0$ & $<4.8$ & $73.4 \pm 24.4$ & \\
\hline & 021908.24 & 1.24 & -035759.87 & 1.86 & $C$ & & $5.5 \pm 0.9$ & $<8.6$ & $<3.3$ & $45.8 \pm 3.7$ & \\
\hline \multirow[t]{2}{*}{ J0219.1-0423 } & 021909.01 & 1.07 & -042322.31 & 1.76 & $M$ & $10.0 \pm 1.1$ & $6.1 \pm 0.8$ & $<3.7$ & $<3.2$ & $153.5 \pm 24.5$ & -0.40 \\
\hline & 021908.85 & 1.17 & -042323.98 & 1.79 & $C$ & & $4.0 \pm 0.7$ & $<6.1$ & $<4.9$ & $103.5 \pm 31.4$ & \\
\hline J0219.2-0552 & 021909.26 & 1.22 & -055243.51 & 1.89 & $S$ & $5.9 \pm 1.3$ & $5.9 \pm 1.3$ & $<8.0$ & $<6.1$ & $31.9 \pm 31.3$ & $<-0.46$ \\
\hline J0219.2-0220 & 021910.29 & 1.08 & -022012.94 & 1.77 & $S$ & $14.8 \pm 2.0$ & $14.8 \pm 2.0$ & $<4.5$ & $<3.8$ & $11.0 \pm 21.8$ & -0.91 \\
\hline \multirow{2}{*}{$\mathrm{J} 0219.2-0344^{*}$} & 021911.09 & 1.08 & -034431.99 & 1.76 & $M$ & $89.7 \pm 9.0$ & $85.1 \pm 9.0$ & $13.0 \pm 1.2$ & $7.8 \pm 0.8$ & $113.3 \pm 3.4$ & -0.63 \\
\hline & 021910.72 & 1.18 & -034440.92 & 1.86 & $C$ & & $4.6 \pm 1.0$ & $<7.0$ & $<6.2$ & $-1.0 \pm 67.2$ & \\
\hline J0219.2-0248 & 021912.64 & 1.24 & -024834.13 & 1.78 & $S$ & $14.2 \pm 2.5$ & $14.2 \pm 2.5$ & $<7.1$ & $<4.6$ & $91.9 \pm 12.3$ & -1.20 \\
\hline J0219.3-0438 & 021916.42 & 1.08 & -043835.86 & 1.76 & $S$ & $5.4 \pm 0.7$ & $5.4 \pm 0.7$ & $<3.7$ & $<3.3$ & $40.0 \pm 35.1$ & -0.67 \\
\hline \multirow[t]{3}{*}{ J0219.3-0552* } & 021918.24 & 1.24 & -055240.08 & 1.87 & $M$ & $118.9 \pm 10.6$ & $67.1 \pm 9.4$ & $16.7 \pm 4.2$ & $8.9 \pm 2.5$ & $44.4 \pm 7.4$ & -0.69 \\
\hline & 021917.83 & 1.21 & -055252.05 & 1.77 & $C$ & & $27.3 \pm 3.9$ & $9.3 \pm 3.4$ & $<4.3$ & $77.0 \pm 7.4$ & \\
\hline & 021918.79 & 1.10 & -055236.99 & 1.77 & $C$ & & $24.6 \pm 3.2$ & $5.1 \pm 2.7$ & $<4.1$ & $44.3 \pm 20.7$ & \\
\hline \multirow[t]{4}{*}{ J0219.3-0244* } & 021920.12 & 1.08 & -024422.75 & 1.76 & $M$ & $205.3 \pm 11.9$ & $71.2 \pm 7.9$ & $8.2 \pm 1.5$ & $5.2 \pm 1.3$ & $30.0 \pm 8.4$ & -0.82 \\
\hline & 021919.13 & 1.06 & -024353.00 & 1.75 & $C$ & & $46.2 \pm 4.9$ & $<2.7$ & $<2.5$ & $167.0 \pm 22.9$ & \\
\hline & 021921.39 & 1.07 & -024402.05 & 1.75 & $C$ & & $44.4 \pm 4.9$ & $4.2 \pm 1.5$ & $<2.8$ & $115.8 \pm 10.6$ & \\
\hline & 021921.22 & 1.22 & -024405.93 & 1.77 & $C$ & & $43.7 \pm 5.6$ & $13.2 \pm 3.3$ & $3.9 \pm 2.3$ & $101.8 \pm 4.2$ & \\
\hline J0219.4-0238 & 021921.15 & 1.14 & -023855.37 & 2.12 & $S$ & $6.3 \pm 1.4$ & $6.3 \pm 1.4$ & $<10.4$ & $<5.3$ & $6.8 \pm 9.3$ & $<-0.51$ \\
\hline J0219.4-0307 & 021923.37 & 1.09 & -030721.40 & 1.77 & $S$ & $14.5 \pm 1.9$ & $14.5 \pm 1.9$ & $3.7 \pm 3.1$ & $<3.9$ & $142.8 \pm 19.8$ & -0.56 \\
\hline J0219.4-0340 & 021923.39 & 1.22 & -034041.78 & 1.81 & $S$ & $4.7 \pm 0.9$ & $4.7 \pm 0.9$ & $<7.2$ & $<5.1$ & $60.4 \pm 16.8$ & $<-0.31$ \\
\hline J0219.4-0300 & 021923.70 & 1.51 & -030010.98 & 2.22 & $S$ & $14.0 \pm 3.1$ & $14.0 \pm 3.1$ & $14.0 \pm 9.1$ & $<5.9$ & $143.4 \pm 7.1$ & $<-1.06$ \\
\hline J0219.4-0548 & 021925.03 & 1.12 & -054806.72 & 1.82 & $S$ & $11.3 \pm 2.0$ & $11.3 \pm 2.0$ & $<6.6$ & $<4.1$ & $147.5 \pm 11.5$ & $<-0.91$ \\
\hline J0219.4-0404 & 021925.65 & 1.21 & -040419.86 & 1.88 & $M$ & $11.7 \pm 1.8$ & $11.7 \pm 1.8$ & $11.4 \pm 4.7$ & $<4.4$ & $140.0 \pm 5.3$ & -0.41 \\
\hline J0219.4-0313 & 021926.54 & 1.18 & -031350.25 & 1.94 & $S$ & $4.8 \pm 1.0$ & $4.8 \pm 1.0$ & $<8.4$ & $<6.1$ & $166.5 \pm 23.8$ & $<-0.32$ \\
\hline J0219.4-0302 & 021926.82 & 1.22 & -030203.68 & 1.90 & $S$ & $5.2 \pm 1.1$ & $5.2 \pm 1.1$ & $<8.5$ & $<5.0$ & $142.9 \pm 13.0$ & $<-0.38$ \\
\hline J0219.5-0229 & 021927.51 & 1.07 & -022957.99 & 1.76 & $S$ & $21.6 \pm 2.5$ & $21.6 \pm 2.5$ & $<3.4$ & $<3.2$ & $0.4 \pm 61.9$ & -0.75 \\
\hline J0219.5-0403 & 021927.53 & 1.12 & -040344.31 & 1.77 & $C$ & $7.4 \pm 1.0$ & $3.9 \pm 0.7$ & $<5.2$ & $<4.0$ & $80.1 \pm 23.7$ & \\
\hline & 021927.55 & 1.32 & -040347.56 & 1.81 & $C$ & & $3.5 \pm 0.8$ & $<8.2$ & $<5.3$ & $68.2 \pm 16.4$ & \\
\hline J0219.5-0539* & 021928.29 & 1.06 & -053949.45 & 1.75 & $M$ & $1412.8 \pm 131.4$ & $1310.4 \pm 131.1$ & $2.9 \pm 0.1$ & $1.8 \pm 0.1$ & $125.1 \pm 2.0$ & -0.84 \\
\hline & 021925.35 & 1.06 & -053913.98 & 1.75 & $C$ & & $76.7 \pm 8.1$ & $2.5 \pm 1.6$ & $<2.4$ & $20.1 \pm 10.5$ & \\
\hline & 021928.77 & 1.06 & -053957.16 & 1.79 & $C$ & & $25.7 \pm 3.4$ & $<5.2$ & $<2.5$ & $178.7 \pm 3.6$ & \\
\hline J0219.5-0251* & 021929.55 & 1.06 & -025111.62 & 1.75 & $M$ & $459.8 \pm 30.9$ & $245.4 \pm 24.7$ & $7.0 \pm 0.3$ & $3.3 \pm 0.3$ & $102.6 \pm 1.6$ & -0.96 \\
\hline & 021930.86 & 1.06 & -025115.18 & 1.75 & $C$ & & $180.9 \pm 18.3$ & $6.4 \pm 0.4$ & $3.2 \pm 0.5$ & $90.8 \pm 2.3$ & \\
\hline & 021930.26 & 1.11 & -025114.09 & 1.81 & $C$ & & $21.1 \pm 3.0$ & $5.8 \pm 3.9$ & $<3.8$ & $32.5 \pm 7.0$ & \\
\hline & 021930.12 & 1.06 & -025110.88 & 1.77 & $C$ & & $12.4 \pm 1.6$ & $<4.1$ & $<2.5$ & $7.8 \pm 6.9$ & \\
\hline J0219.5-0342 & 021932.73 & 1.09 & -034257.39 & 1.78 & $S$ & $7.4 \pm 1.0$ & $7.4 \pm 1.0$ & $<4.8$ & $<3.8$ & $29.7 \pm 16.3$ & $<-0.61$ \\
\hline J0219.6-0305 & 021934.47 & 1.33 & -030541.55 & 1.99 & $S$ & $4.9 \pm 1.1$ & $4.9 \pm 1.1$ & $<10.3$ & $<5.0$ & $141.1 \pm 8.0$ & $<-0.34$ \\
\hline J0219.6-0414 & 021934.89 & 1.36 & -041436.70 & 2.00 & $S$ & $3.4 \pm 0.7$ & $3.4 \pm 0.7$ & $<10.7$ & $<4.1$ & $41.0 \pm 4.8$ & $<-0.08$ \\
\hline J0219.6-0418 & 021935.69 & 1.06 & -041842.92 & 1.75 & $S$ & $28.8 \pm 3.0$ & $28.8 \pm 3.0$ & $<2.1$ & $<2.1$ & $167.8 \pm 51.2$ & -0.73 \\
\hline J0219.6-0306 & 021936.96 & 1.10 & -030650.28 & 1.80 & $S$ & $7.7 \pm 1.2$ & $7.7 \pm 1.2$ & $<5.7$ & $<4.5$ & $18.4 \pm 20.6$ & -0.75 \\
\hline J0219.6-0301 & 021937.74 & 1.07 & -030148.55 & 1.76 & $M$ & $71.9 \pm 5.3$ & $33.1 \pm 3.7$ & $6.8 \pm 1.5$ & $<2.9$ & $150.7 \pm 5.5$ & -0.90 \\
\hline & 021938.84 & 1.08 & -030206.45 & 1.76 & $C$ & & $32.8 \pm 3.7$ & $7.1 \pm 1.5$ & $<3.0$ & $136.1 \pm 6.0$ & \\
\hline & 021938.36 & 1.22 & -030159.90 & 1.84 & $C$ & & $6.1 \pm 1.2$ & $<7.3$ & $<5.8$ & $52.4 \pm 32.4$ & \\
\hline J0219.6-0349 & 021937.94 & 1.12 & -034918.18 & 1.79 & $S$ & $6.6 \pm 1.0$ & $6.6 \pm 1.0$ & $<5.8$ & $<4.0$ & $41.5 \pm 9.9$ & $<-0.54$ \\
\hline J0219.7-0511 & 021939.07 & 1.11 & -051136.23 & 1.86 & $S$ & $4.2 \pm 0.8$ & $4.2 \pm 0.8$ & $<7.3$ & $<4.1$ & $21.3 \pm 10.4$ & $<-0.23$ \\
\hline J0219.7-0341 & 021941.88 & 1.24 & -034155.77 & 1.93 & $S$ & $4.1 \pm 0.9$ & $4.1 \pm 0.9$ & $<8.8$ & $<5.8$ & $145.3 \pm 16.4$ & 0.07 \\
\hline J0219.7-0400 & 021942.78 & 1.45 & -040037.10 & 3.24 & $M$ & $150.7 \pm 12.5^{*}$ & $44.6 \pm 6.8$ & $44.4 \pm 13.13$ & $11.0 \pm 6.5$ & $170.9 \pm 4.3$ & -1.16 \\
\hline & 021941.16 & 2.15 & -040025.66 & 2.27 & $C$ & & $35.1 \pm 6.1$ & $29.0 \pm 9.93$ & $<11.8$ & $60.7 \pm 11.3$ & \\
\hline & 021942.02 & 1.70 & -040046.00 & 2.13 & $C$ & & $20.7 \pm 4.0$ & $17.8 \pm 7.09$ & $<13.7$ & $122.6 \pm 32.4$ & \\
\hline & 021939.16 & 2.16 & -040019.82 & 2.39 & $C$ & & $10.3 \pm 3.2$ & $16.4 \pm 10.92$ & $<5.3$ & $123.7 \pm 26.6$ & \\
\hline & 021941.21 & 1.89 & -04004.73 & 2.25 & $C$ & & $7.8 \pm 2.5$ & $<20.4$ & $<14.0$ & $71.6 \pm 84.1$ & \\
\hline & 021940.36 & 2.04 & -040013.37 & 3.35 & $C$ & & $7.5 \pm 3.0$ & $<34.1$ & $<12.4$ & $19.8 \pm 18.6$ & \\
\hline & 021939.37 & 1.42 & -035948.97 & 2.07 & $C$ & & $6.4 \pm 1.9$ & $<15.6$ & $<3.2$ & $3.9 \pm 53.7$ & \\
\hline & 021939.57 & 2.03 & -04002.66 & 2.18 & $C$ & & $6.1 \pm 2.2$ & $<21.2$ & $<7.5$ & $100.2 \pm 38.4$ & \\
\hline & 021938.11 & 1.58 & -040015.96 & 3.38 & $C$ & & $4.6 \pm 2.0$ & $<33.6$ & $\ldots$ & $13.9 \pm 9.9$ & \\
\hline & 021939.95 & 5.69 & -035951.74 & 5.47 & $C$ & & $4.5 \pm 2.8$ & $<68.6$ & $<10.2$ & $47.3 \pm 7.7$ & \\
\hline J0219.7-0604 & 021942.77 & 1.08 & -060425.85 & 1.81 & $M$ & $18.3 \pm 1.6$ & $7.7 \pm 1.3$ & $<6.0$ & $<3.9$ & $6.3 \pm 11.7$ & $<-1.24$ \\
\hline & 021941.83 & 1.07 & -060435.84 & 1.79 & $C$ & & $5.4 \pm 0.7$ & $<5.4$ & $<2.6$ & $13.7 \pm 3.9$ & \\
\hline & 021942.16 & 1.08 & -060423.61 & 1.79 & $C$ & & $5.1 \pm 0.7$ & $<5.1$ & $<3.3$ & $164.6 \pm 8.9$ & \\
\hline J0219.7-0326 & 021943.03 & 1.08 & -032627.36 & 1.76 & $S$ & $17.0 \pm 1.9$ & $17.0 \pm 1.9$ & $3.8 \pm 1.8$ & $<3.1$ & $69.6 \pm 13.3$ & -0.61 \\
\hline
\end{tabular}


C. Tasse et al.: The low-frequency counterpart of the XMM large scale structure survey, Online Material $p$ 11

Table A.1. continued.

\begin{tabular}{|c|c|c|c|c|c|c|c|c|c|c|c|}
\hline Name & $\begin{array}{c}\text { RA } \\
\text { J2000 }\end{array}$ & $\begin{array}{c}\sigma_{\alpha} \\
\operatorname{arcsec}\end{array}$ & $\begin{array}{c}\text { Dec } \\
\text { J2000 }\end{array}$ & $\begin{array}{c}\sigma_{\delta} \\
\operatorname{arcsec}\end{array}$ & Type & $\begin{array}{l}S_{\text {int }} \\
\text { mJy }\end{array}$ & $\begin{array}{c}S_{\text {comp }} \\
\text { mJy }\end{array}$ & $\begin{array}{c}\text { Major axis } \\
\text { arcsec }\end{array}$ & $\begin{array}{c}\text { Minor axis } \\
\text { arcsec }\end{array}$ & $\begin{array}{c}\text { Position } \\
\text { Angle }\end{array}$ & $\begin{array}{c}\text { Spectral } \\
\text { index } \alpha_{325}^{1400}\end{array}$ \\
\hline \multirow[t]{2}{*}{ J0219.7-0516 } & 021943.54 & 1.07 & -051624.39 & 1.76 & $M$ & $27.7 \pm 2.4$ & $17.4 \pm 2.0$ & $4.1 \pm 2.0$ & $<3.0$ & $157.1 \pm 7.8$ & -0.85 \\
\hline & 021943.37 & 1.08 & -051620.35 & 1.76 & $C$ & & $10.4 \pm 1.3$ & $<4.3$ & $<2.7$ & $40.8 \pm 5.3$ & \\
\hline J0219.7-0332 & 021944.43 & 1.09 & -033216.19 & 1.76 & $S$ & $9.6 \pm 1.2$ & $9.6 \pm 1.2$ & $<4.3$ & $<3.5$ & $47.9 \pm 14.7$ & -0.49 \\
\hline \multirow{2}{*}{ J0219.7-0448 } & 021944.65 & 1.28 & -044846.62 & 1.77 & $M$ & $16.4 \pm 2.0$ & $12.4 \pm 1.8$ & $10.6 \pm 4.1$ & $<4.3$ & $96.1 \pm 5.6$ & -0.72 \\
\hline & 021944.93 & 1.15 & -044844.54 & 1.80 & $C$ & & $4.0 \pm 0.7$ & $<6.3$ & $<4.6$ & $47.6 \pm 19.8$ & \\
\hline \multirow[t]{2}{*}{ J0219.8-0453 } & 021945.33 & 1.09 & -045334.57 & 1.77 & $M$ & $25.4 \pm 2.7$ & $14.1 \pm 1.7$ & $5.3 \pm 2.2$ & $<3.8$ & $52.7 \pm 16.3$ & -0.51 \\
\hline & 021945.82 & 1.23 & -045342.49 & 2.17 & $C$ & & $11.3 \pm 2.1$ & $14.8 \pm 7.2$ & $<5.5$ & $20.9 \pm 6.0$ & \\
\hline J0219.8-0456 & 021945.73 & 1.26 & -045620.34 & 1.82 & $S$ & $3.5 \pm 0.7$ & $3.5 \pm 0.7$ & $<7.8$ & $<5.2$ & $59.6 \pm 17.0$ & $<-0.11$ \\
\hline J0219.8-0549 & 021948.80 & 1.13 & -054923.99 & 2.04 & $S$ & $8.2 \pm 1.5$ & $8.2 \pm 1.5$ & $<9.9$ & $<3.2$ & $160.4 \pm 3.2$ & $<-0.69$ \\
\hline J0219.8-0437 & 021950.66 & 1.12 & -043720.27 & 1.78 & $S$ & $3.3 \pm 0.6$ & $3.3 \pm 0.6$ & $<5.4$ & $<4.2$ & $124.6 \pm 25.9$ & -0.30 \\
\hline J0219.9-0158 & 021951.57 & 1.10 & -015854.70 & 1.79 & $S$ & $11.3 \pm 1.9$ & $11.3 \pm 1.9$ & $<5.4$ & $<4.2$ & $156.1 \pm 23.6$ & -0.46 \\
\hline J0219.9-0444 & 021951.73 & 1.12 & -044435.89 & 1.77 & $S$ & $6.2 \pm 0.9$ & $6.2 \pm 0.9$ & $<5.4$ & $<4.1$ & $61.9 \pm 15.1$ & $<-0.50$ \\
\hline \multirow[t]{3}{*}{ J0219.9-0604 } & 021952.27 & 1.07 & -060425.41 & 1.81 & $M$ & $12.0 \pm 1.1$ & $5.2 \pm 0.7$ & $<5.8$ & $<2.4$ & $171.9 \pm 3.0$ & $<-0.95$ \\
\hline & 021952.62 & 1.20 & -060431.33 & 1.81 & $C$ & & $3.4 \pm 0.6$ & $<7.3$ & $<4.1$ & $128.0 \pm 10.0$ & \\
\hline & 021953.92 & 1.11 & -060431.40 & 2.10 & $C$ & & $3.4 \pm 0.6$ & $<10.4$ & $<3.0$ & $165.9 \pm 2.6$ & \\
\hline J0219.9-0532 & 021952.74 & 1.13 & -053250.37 & 1.89 & $S$ & $6.1 \pm 1.2$ & $6.1 \pm 1.2$ & $<7.7$ & $<4.9$ & $14.7 \pm 13.6$ & $<-0.49$ \\
\hline \multirow{2}{*}{ J0219.9-0518* } & 021953.47 & 1.06 & -051837.08 & 1.75 & $M$ & $74.2 \pm 5.9$ & $53.5 \pm 5.4$ & $1.8 \pm 0.9$ & $<1.6$ & $33.4 \pm 6.8$ & -0.89 \\
\hline & 021952.73 & 1.07 & -051807.83 & 1.75 & $C$ & & $20.7 \pm 2.2$ & $<3.0$ & $<2.6$ & $23.1 \pm 10.7$ & \\
\hline J0219.9-0211 & 021953.92 & 1.22 & -021129.32 & 1.79 & $S$ & $18.1 \pm 2.9$ & $18.1 \pm 2.9$ & $7.2 \pm 4.3$ & $<4.9$ & $70.7 \pm 12.7$ & $<-1.23$ \\
\hline J0219.9-0549 & 021954.53 & 1.10 & -054924.41 & 1.79 & $S$ & $11.5 \pm 1.8$ & $11.5 \pm 1.8$ & $<5.3$ & $<4.4$ & $28.9 \pm 27.6$ & -0.84 \\
\hline \multirow{3}{*}{ J0219.9-0527 } & 021955.35 & 1.08 & -052748.54 & 1.76 & $M$ & $40.6 \pm 3.3$ & $15.9 \pm 1.9$ & $<3.8$ & $<3.5$ & $41.0 \pm 29.7$ & 0.08 \\
\hline & 021956.76 & 1.14 & -052825.12 & 1.84 & $C$ & & $14.4 \pm 2.2$ & $8.0 \pm 3.6$ & $4.5 \pm 3.6$ & $17.9 \pm 20.8$ & \\
\hline & 021955.71 & 1.12 & -052751.68 & 1.79 & $C$ & & $10.4 \pm 1.5$ & $<5.1$ & $\ldots$ & $\ldots$ & \\
\hline J0220.0-0322 & 021957.16 & 1.09 & -032209.99 & 1.77 & $M$ & $10.2 \pm 1.2$ & $5.3 \pm 0.9$ & $<4.7$ & $<3.8$ & $32.7 \pm 29.4$ & -0.81 \\
\hline & 021956.96 & 1.08 & -032210.00 & 1.94 & $C$ & & $4.9 \pm 0.8$ & $<8.3$ & $<3.1$ & $9.7 \pm 3.9$ & \\
\hline J0220.0-0447* & 021957.25 & 1.06 & -044753.48 & 1.75 & $M$ & $126.2 \pm 12.2$ & $122.0 \pm 12.2$ & $4.0 \pm 0.2$ & $<1.0$ & $32.7 \pm 1.2$ & -0.79 \\
\hline & 021956.72 & 1.07 & -044743.52 & 1.85 & $C$ & & $4.2 \pm 0.7$ & $<6.7$ & $<2.8$ & $8.2 \pm 4.5$ & \\
\hline J0220.0-0523 & 021957.29 & 1.20 & -052350.48 & 1.83 & $M$ & $9.8 \pm 1.2$ & $5.4 \pm 0.9$ & $<7.8$ & $<3.2$ & $46.5 \pm 4.4$ & -0.68 \\
\hline & 021957.20 & 1.10 & -052348.12 & 1.78 & $C$ & & $4.4 \pm 0.8$ & $<4.8$ & $<4.4$ & $-1.0 \pm 87.5$ & \\
\hline J0220.0-0349 & 021959.73 & 1.28 & -034940.33 & 1.84 & $S$ & $3.8 \pm 0.8$ & $3.8 \pm 0.8$ & $<8.3$ & $<5.1$ & $53.2 \pm 13.4$ & $<-0.17$ \\
\hline J0220.0-0522 & 022001.60 & 1.08 & -052218.38 & 1.76 & $M$ & $19.2 \pm 1.8$ & $12.4 \pm 1.4$ & $<3.8$ & $<2.7$ & $57.2 \pm 7.1$ & -0.13 \\
\hline & 022003.23 & 1.08 & -052223.98 & 1.81 & $C$ & & $6.8 \pm 1.0$ & $<6.1$ & $<3.3$ & $18.1 \pm 5.8$ & \\
\hline J0220.1-0349 & 022008.51 & 1.11 & -034855.14 & 1.78 & $M$ & $17.0 \pm 1.8$ & $13.5 \pm 1.7$ & $7.7 \pm 2.7$ & $<3.1$ & $48.3 \pm 3.8$ & 0.08 \\
\hline & 022007.96 & 1.35 & -034903.50 & 1.86 & $C$ & & $3.4 \pm 0.7$ & $<9.4$ & $<3.4$ & $54.1 \pm 4.3$ & \\
\hline J0220.1-0545 & 022008.73 & 1.14 & -054558.88 & 1.80 & $M$ & $42.4 \pm 3.8$ & $23.4 \pm 3.1$ & $9.3 \pm 3.4$ & $<3.0$ & $46.3 \pm 2.9$ & -0.76 \\
\hline & 022009.01 & 1.07 & -054553.38 & 1.76 & $C$ & & $19.0 \pm 2.2$ & $<3.2$ & $\ldots$ & $\ldots$ & \\
\hline J0220.2-0404 & 022010.57 & 1.15 & -040422.31 & 1.83 & $S$ & $5.0 \pm 0.8$ & $5.0 \pm 0.8$ & $<6.8$ & $<5.0$ & $35.7 \pm 15.6$ & $<-0.34$ \\
\hline J0220.2-0305 & 022011.49 & 1.08 & -030534.51 & 1.75 & $M$ & $15.0 \pm 1.4$ & $10.0 \pm 1.3$ & $<3.9$ & $<2.9$ & $102.6 \pm 9.6$ & $<-1.10$ \\
\hline & 022011.44 & 1.11 & -030531.60 & 1.75 & $C$ & & $5.0 \pm 0.7$ & $<4.8$ & $<2.4$ & $103.0 \pm 5.3$ & \\
\hline J0220.2-0421 & 022013.16 & 1.07 & -042149.16 & 1.76 & $S$ & $11.9 \pm 1.3$ & $11.9 \pm 1.3$ & $<3.2$ & $<2.5$ & $42.4 \pm 7.5$ & -0.50 \\
\hline J0220.2-0441 & 022013.60 & 1.10 & -044115.57 & 1.87 & $S$ & $3.6 \pm 0.7$ & $3.6 \pm 0.7$ & $<7.3$ & $<4.6$ & $4.9 \pm 12.5$ & $<-0.12$ \\
\hline J0220.3-0346 & 022016.92 & 1.09 & -034606.17 & 1.80 & $S$ & $5.4 \pm 0.8$ & $5.4 \pm 0.8$ & $<5.6$ & $<4.0$ & $177.6 \pm 13.6$ & -0.61 \\
\hline J0220.3-0518 & 022017.42 & 1.08 & -051851.82 & 1.77 & $S$ & $6.8 \pm 0.9$ & $6.8 \pm 0.9$ & $<4.3$ & $<3.2$ & $40.1 \pm 11.8$ & $<-0.56$ \\
\hline J0220.3-0324 & 022018.33 & 1.13 & -032423.61 & 1.79 & $S$ & $7.2 \pm 1.1$ & $7.2 \pm 1.1$ & $<6.1$ & $<4.3$ & $135.1 \pm 13.1$ & $<-0.60$ \\
\hline J0220.3-0557 & 022018.80 & 1.19 & -055731.70 & 1.88 & $S$ & $7.5 \pm 1.3$ & $7.5 \pm 1.3$ & $<8.3$ & $<3.6$ & $143.1 \pm 5.1$ & -0.52 \\
\hline J0220.3-0336 & 022018.96 & 1.30 & -033632.30 & 1.92 & $S$ & $3.5 \pm 0.7$ & $3.5 \pm 0.7$ & $<9.7$ & $<3.7$ & $43.1 \pm 4.8$ & $<-0.11$ \\
\hline J0220.3-0237 & 022019.67 & 1.10 & -023757.24 & 1.77 & $S$ & $11.2 \pm 1.6$ & $11.2 \pm 1.6$ & $<4.6$ & $<3.8$ & $54.6 \pm 20.6$ & -0.16 \\
\hline J0220.4-0448 & 022024.84 & 1.17 & -044816.32 & 1.84 & $S$ & $3.2 \pm 0.7$ & $3.2 \pm 0.7$ & $<6.5$ & $\ldots$ & $\ldots$ & $<-0.04$ \\
\hline J0220.5-0348 & 022027.18 & 1.22 & -034843.12 & 2.01 & $S$ & $3.6 \pm 0.8$ & $3.6 \pm 0.8$ & $<9.8$ & $<5.0$ & $152.2 \pm 9.1$ & $<-0.12$ \\
\hline J0220.5-0330 & 022027.41 & 1.12 & -033049.06 & 1.78 & $S$ & $6.4 \pm 0.9$ & $6.4 \pm 0.9$ & $<5.4$ & $<3.9$ & $54.8 \pm 12.6$ & 0.28 \\
\hline J0220.5-0442 & 022027.83 & 1.08 & -044219.12 & 1.76 & $S$ & $6.9 \pm 0.9$ & $6.9 \pm 0.9$ & $<3.9$ & $<3.5$ & $23.9 \pm 26.8$ & $<-0.57$ \\
\hline J0220.5-0422 & 022027.84 & 1.12 & -042251.92 & 1.78 & $S$ & $5.2 \pm 0.8$ & $5.2 \pm 0.8$ & $<5.2$ & $<4.7$ & $53.1 \pm 50.4$ & $<-0.37$ \\
\hline J0220.5-0547 & 022028.85 & 1.13 & -054736.74 & 1.78 & $S$ & $6.3 \pm 1.1$ & $6.3 \pm 1.1$ & $<5.5$ & $<4.8$ & $117.0 \pm 45.6$ & -0.40 \\
\hline J0220.5-0603 & 022029.66 & 1.08 & -060310.15 & 1.78 & $M$ & $18.5 \pm 1.9$ & $9.9 \pm 1.4$ & $<5.0$ & $<3.3$ & $17.8 \pm 9.7$ & -0.87 \\
\hline & 022029.64 & 1.15 & -060313.46 & 1.78 & $C$ & & $8.6 \pm 1.3$ & $<6.3$ & $<3.0$ & $54.0 \pm 4.8$ & \\
\hline J0220.5-0251 & 022030.81 & 1.08 & -025105.83 & 1.77 & $S$ & $16.8 \pm 2.0$ & $16.8 \pm 2.0$ & $4.0 \pm 2.5$ & $<3.4$ & $157.3 \pm 12.0$ & -0.49 \\
\hline J0220.5-0450 & 022031.97 & 1.09 & -045003.56 & 1.78 & $S$ & $5.5 \pm 0.8$ & $5.5 \pm 0.8$ & $<5.3$ & $<3.1$ & $140.7 \pm 6.1$ & $<-0.42$ \\
\hline J0220.6-0414 & 022033.06 & 1.12 & -041416.58 & 1.80 & $S$ & $3.5 \pm 0.6$ & $3.5 \pm 0.6$ & $<6.1$ & $<3.5$ & $40.5 \pm 9.6$ & $<-0.11$ \\
\hline J0220.6-0257 & 022033.26 & 1.18 & -025743.33 & 1.86 & $S$ & $5.1 \pm 1.1$ & $5.1 \pm 1.1$ & $<7.5$ & $<5.1$ & $35.4 \pm 18.2$ & $<-0.37$ \\
\hline J0220.6-0517 & 022034.47 & 1.38 & -051747.43 & 1.87 & $S$ & $4.6 \pm 0.8$ & $4.6 \pm 0.8$ & $<9.7$ & $<3.7$ & $54.5 \pm 4.2$ & $<-0.29$ \\
\hline J0220.6-0410 & 022034.71 & 1.21 & -041059.57 & 1.87 & $S$ & $4.1 \pm 0.8$ & $4.1 \pm 0.8$ & $<7.1$ & $\ldots$ & $\ldots$ & $<-0.21$ \\
\hline J0220.6-0409 & 022034.81 & 1.15 & -040954.54 & 1.82 & $S$ & $3.9 \pm 0.7$ & $3.9 \pm 0.7$ & $<6.7$ & $<4.7$ & $142.0 \pm 17.9$ & $<-0.17$ \\
\hline J0220.6-0513 & 022035.34 & 1.06 & -051400.39 & 1.75 & $S$ & $38.1 \pm 3.9$ & $38.1 \pm 3.9$ & $<1.9$ & $<1.6$ & $30.6 \pm 4.4$ & -0.92 \\
\hline J0220.6-0342 & 022035.35 & 1.27 & -034245.56 & 1.81 & $S$ & $4.6 \pm 0.9$ & $4.6 \pm 0.9$ & $<7.6$ & $<5.4$ & $68.6 \pm 18.3$ & $<-0.29$ \\
\hline J0220.6-0240 & 022035.86 & 1.06 & -024025.41 & 1.75 & $S$ & $48.5 \pm 5.0$ & $48.5 \pm 5.0$ & $<2.3$ & $<2.3$ & $48.4 \pm 30.6$ & -0.64 \\
\hline J0220.6-0408 & 022036.13 & 1.21 & -040809.39 & 1.81 & $M$ & $6.9 \pm 0.9$ & $3.7 \pm 0.7$ & $<7.5$ & $<4.0$ & $54.9 \pm 8.2$ & $<-0.57$ \\
\hline & 022036.03 & 1.12 & -040806.05 & 1.83 & $C$ & & $3.2 \pm 0.6$ & $<6.7$ & $<4.3$ & $27.7 \pm 14.6$ & \\
\hline J0220.6-0543 & 022036.26 & 1.06 & -054329.86 & 1.75 & $M$ & $39.8 \pm 3.0$ & $20.6 \pm 2.2$ & $<2.8$ & $<2.5$ & $6.5 \pm 15.3$ & -0.82 \\
\hline & 022037.22 & 1.08 & -054330.61 & 1.76 & $C$ & & $13.7 \pm 1.7$ & $<3.8$ & $<3.4$ & $39.2 \pm 22.5$ & \\
\hline & 022036.52 & 1.11 & -054328.08 & 1.87 & $C$ & & $5.5 \pm 1.1$ & $<7.2$ & $<4.6$ & $11.8 \pm 13.9$ & \\
\hline J0220.6-0312 & 022036.70 & 1.07 & -031240.97 & 1.75 & $S$ & $20.3 \pm 2.2$ & $20.3 \pm 2.2$ & $<3.0$ & $<2.6$ & $30.3 \pm 10.7$ & -0.75 \\
\hline J0220.6-0437 & 022038.95 & 1.08 & -043727.20 & 1.76 & $M$ & $24.4 \pm 2.2$ & $15.2 \pm 1.7$ & $5.3 \pm 1.7$ & $<3.3$ & $144.2 \pm 13.3$ & -0.63 \\
\hline & 022038.62 & 1.12 & -043719.01 & 1.78 & $C$ & & $9.3 \pm 1.3$ & $5.3 \pm 3.1$ & $<4.4$ & $53.9 \pm 19.2$ & \\
\hline J0220.7-0222 & 022042.46 & 1.17 & -022202.71 & 1.78 & $S$ & $13.8 \pm 2.0$ & $13.8 \pm 2.0$ & $5.5 \pm 4.2$ & $<3.9$ & $65.2 \pm 7.8$ & -0.48 \\
\hline
\end{tabular}


C. Tasse et al.: The low-frequency counterpart of the XMM large scale structure survey, Online Material $p 12$

Table A.1. continued.

\begin{tabular}{|c|c|c|c|c|c|c|c|c|c|c|c|}
\hline Name & $\begin{array}{c}\text { RA } \\
\text { J2000 }\end{array}$ & $\begin{array}{c}\sigma_{\alpha} \\
\operatorname{arcsec}\end{array}$ & $\begin{array}{c}\text { Dec } \\
\text { J2000 }\end{array}$ & $\begin{array}{c}\sigma_{\delta} \\
\operatorname{arcsec}\end{array}$ & Type & $\begin{array}{l}S_{\text {int }} \\
\mathrm{mJy}\end{array}$ & $\begin{array}{c}S_{\text {comp }} \\
\text { mJy }\end{array}$ & $\begin{array}{c}\text { Major axis } \\
\text { arcsec }\end{array}$ & $\begin{array}{c}\text { Minor axis } \\
\text { arcsec }\end{array}$ & $\begin{array}{c}\text { Position } \\
\text { Angle }\end{array}$ & $\begin{array}{c}\text { Spectral } \\
\text { index } \alpha_{325}^{1400}\end{array}$ \\
\hline J0220.7-0339 & 022044.17 & 1.10 & -033948.03 & 1.83 & $S$ & $11.2 \pm 1.7$ & $11.2 \pm 1.7$ & $6.2 \pm 4.0$ & $<4.2$ & $165.7 \pm 9.1$ & -0.63 \\
\hline J0220.7-0518 & 022044.18 & 1.07 & -051806.61 & 1.80 & $S$ & $6.7 \pm 0.9$ & $6.7 \pm 0.9$ & $<5.7$ & $<2.9$ & $11.5 \pm 4.3$ & $<-0.55$ \\
\hline J0220.8-0321 & 022045.64 & 1.13 & -032130.90 & 1.77 & $S$ & $9.5 \pm 1.4$ & $9.5 \pm 1.4$ & $<5.3$ & $<4.4$ & $94.6 \pm 23.0$ & -0.42 \\
\hline J0220.8-0335 & 022045.67 & 1.21 & -033551.86 & 1.85 & $S$ & $4.9 \pm 1.0$ & $4.9 \pm 1.0$ & $<7.6$ & $<5.5$ & $43.8 \pm 21.9$ & $<-0.33$ \\
\hline J0220.8-0237 & 022048.18 & 1.09 & -023726.42 & 1.80 & $S$ & $12.2 \pm 1.9$ & $12.2 \pm 1.9$ & $<5.6$ & $<4.0$ & $178.1 \pm 13.7$ & -0.97 \\
\hline J0220.8-0333* & 022049.52 & 1.06 & -033317.90 & 1.75 & $S$ & $224.5 \pm 22.5$ & $224.5 \pm 22.5$ & $7.5 \pm 0.2$ & $3.2 \pm 0.2$ & $73.7 \pm 0.9$ & -0.53 \\
\hline J0220.8-0553 & 022049.62 & 1.07 & -055356.16 & 1.76 & $S$ & $19.0 \pm 2.2$ & $19.0 \pm 2.2$ & $<3.7$ & $<3.1$ & $22.7 \pm 12.2$ & -0.55 \\
\hline J0220.8-0537 & 022050.38 & 1.07 & -053715.14 & 1.76 & $S$ & $14.7 \pm 1.6$ & $14.7 \pm 1.6$ & $<3.2$ & $<2.5$ & $31.5 \pm 7.5$ & -1.03 \\
\hline \multirow[t]{2}{*}{ J0220.8-0510 } & 022050.63 & 1.15 & -051019.70 & 1.88 & $M$ & $12.0 \pm 1.6$ & $8.7 \pm 1.4$ & $8.8 \pm 4.1$ & $<5.7$ & $178.5 \pm 18.3$ & -0.37 \\
\hline & 022050.98 & 1.13 & -050959.93 & 1.91 & $C$ & & $3.3 \pm 0.7$ & $<8.0$ & $<4.8$ & $14.7 \pm 13.1$ & \\
\hline \multirow[t]{2}{*}{ J0220.8-0340 } & 022051.12 & 1.50 & -034055.53 & 1.80 & $M$ & $65.6 \pm 5.8$ & $37.7 \pm 4.9$ & $22.4 \pm 5.5$ & $<3.3$ & $70.5 \pm 1.5$ & -1.14 \\
\hline & 022050.22 & 1.08 & -034059.87 & 1.76 & $C$ & & $27.8 \pm 3.1$ & $5.1 \pm 1.6$ & $<2.9$ & $61.8 \pm 7.3$ & \\
\hline J0220.9-0512 & 022051.69 & 1.07 & -051237.11 & 1.77 & $S$ & $8.6 \pm 1.1$ & $8.6 \pm 1.1$ & $<4.5$ & $<3.2$ & $3.6 \pm 8.0$ & 0.01 \\
\hline \multirow[t]{2}{*}{ J0220.9-0413 } & 022051.77 & 1.07 & -041345.79 & 1.79 & $M$ & $8.9 \pm 1.0$ & $5.7 \pm 0.7$ & $<5.2$ & $<2.2$ & $166.0 \pm 2.8$ & $<-0.75$ \\
\hline & 022051.84 & 1.42 & -041358.13 & 1.79 & $C$ & & $3.2 \pm 0.7$ & $<9.0$ & $<4.9$ & $97.3 \pm 10.9$ & \\
\hline \multirow[t]{2}{*}{ J0220.9-0601* } & 022051.96 & 1.07 & -060118.92 & 1.76 & $M$ & $79.7 \pm 7.7$ & $70.4 \pm 7.5$ & $9.5 \pm 1.2$ & $3.6 \pm 1.1$ & $135.1 \pm 3.1$ & -0.72 \\
\hline & 022051.42 & 1.15 & -060113.45 & 1.86 & $C$ & & $9.3 \pm 1.5$ & $<7.7$ & $<3.6$ & $33.2 \pm 5.0$ & \\
\hline J0220.9-0252 & 022052.04 & 1.48 & -025301.68 & 1.97 & $S$ & $12.7 \pm 2.8$ & $12.7 \pm 2.8$ & $10.0 \pm 7.8$ & $<6.3$ & $129.9 \pm 11.7$ & -0.96 \\
\hline J0220.9-0529 & 022053.20 & 1.09 & -052908.05 & 1.78 & $S$ & $6.3 \pm 0.9$ & $6.3 \pm 0.9$ & $<4.8$ & $<4.0$ & $25.4 \pm 26.6$ & $<-0.51$ \\
\hline J0220.9-0219 & 022054.25 & 1.28 & -021917.50 & 1.80 & $S$ & $14.3 \pm 2.9$ & $14.3 \pm 2.9$ & $<7.6$ & $<5.5$ & $88.9 \pm 21.4$ & $<-1.07$ \\
\hline \multirow{2}{*}{ J0220.9-0333 } & 022055.51 & 1.06 & -033331.72 & 1.75 & $M$ & $43.8 \pm 4.2$ & $38.7 \pm 4.0$ & $<2.2$ & $\ldots$ & & -0.38 \\
\hline & 022055.67 & 1.24 & -033350.24 & 1.90 & $C$ & & $5.1 \pm 1.1$ & $<8.3$ & $<6.1$ & $36.9 \pm 24.9$ & \\
\hline \multirow[t]{4}{*}{ J0220.9-0348* } & 022055.77 & 1.06 & -034834.89 & 1.75 & $M$ & $402.2 \pm 35.1$ & $348.6 \pm 34.9$ & $6.4 \pm 0.1$ & $2.9 \pm 0.2$ & $76.4 \pm 0.7$ & -1.11 \\
\hline & 022055.29 & 1.06 & -034836.97 & 1.76 & $C$ & & $27.6 \pm 2.9$ & $5.2 \pm 1.8$ & $<1.1$ & $168.9 \pm 0.5$ & \\
\hline & 022055.10 & 1.06 & -034837.04 & 1.76 & $C$ & & $19.9 \pm 2.2$ & $<3.6$ & $<2.1$ & $1.0 \pm 2.8$ & \\
\hline & 022055.60 & 1.53 & -034847.00 & 1.76 & $C$ & & $6.1 \pm 1.1$ & $<9.8$ & $<3.4$ & $84.2 \pm 3.4$ & \\
\hline J0221.0-0358 & 022057.53 & 1.10 & -035848.60 & 1.77 & $M$ & $24.5 \pm 2.5$ & $17.6 \pm 2.2$ & $6.6 \pm 2.6$ & $<3.2$ & $47.4 \pm 5.2$ & -1.10 \\
\hline & 022057.07 & 1.08 & -035853.47 & 1.78 & $C$ & & $6.9 \pm 1.0$ & $<4.9$ & $<3.6$ & $156.4 \pm 15.2$ & \\
\hline J0221.0-0413 & 022058.71 & 1.37 & -041322.71 & 1.86 & $S$ & $4.3 \pm 0.8$ & $4.3 \pm 0.8$ & $<9.4$ & $<4.4$ & $56.3 \pm 6.2$ & $<-0.25$ \\
\hline J0221.0-0535 & 022100.34 & 1.16 & -053530.77 & 1.82 & $S$ & $5.4 \pm 1.0$ & $5.4 \pm 1.0$ & $<6.4$ & $<5.6$ & $150.2 \pm 47.0$ & -0.18 \\
\hline J0221.0-0500 & 022101.95 & 1.11 & -050048.10 & 1.75 & $M$ & $12.1 \pm 1.2$ & $8.1 \pm 1.0$ & $<4.8$ & $<2.7$ & $95.3 \pm 4.1$ & -0.70 \\
\hline & 022101.91 & 1.14 & -050045.20 & 1.75 & $C$ & & $4.1 \pm 0.6$ & $<5.5$ & $<2.7$ & $81.1 \pm 5.0$ & \\
\hline J0221.0-0240 & 022102.27 & 1.08 & -024027.90 & 1.77 & $S$ & $23.4 \pm 2.9$ & $23.4 \pm 2.9$ & $3.4 \pm 2.6$ & $\ldots$ & $\ldots$ & -0.57 \\
\hline J0221.1-0253 & 022102.95 & 1.09 & -025313.83 & 1.77 & $S$ & $12.7 \pm 1.7$ & $12.7 \pm 1.7$ & $<4.5$ & $<3.8$ & $128.5 \pm 20.1$ & -0.97 \\
\hline J0221.1-0246* & 022103.97 & 1.08 & -024640.03 & 1.77 & $M$ & $127.0 \pm 8.9$ & $60.6 \pm 6.7$ & $11.1 \pm 1.7$ & $4.5 \pm 1.2$ & $156.9 \pm 3.5$ & -0.74 \\
\hline & 022103.61 & 1.10 & -024620.73 & 1.83 & $C$ & & $34.6 \pm 4.3$ & $13.1 \pm 3.2$ & $<3.1$ & $152.7 \pm 2.3$ & \\
\hline & 022103.30 & 1.07 & -024619.63 & 1.81 & $C$ & & $31.8 \pm 3.9$ & $10.4 \pm 2.6$ & $<3.4$ & $177.7 \pm 3.9$ & \\
\hline J0221.2-0511 & 022110.94 & 1.11 & -051120.79 & 1.79 & $M$ & $23.5 \pm 2.4$ & $13.5 \pm 1.7$ & $8.8 \pm 2.9$ & $<3.0$ & $43.3 \pm 3.1$ & -0.54 \\
\hline & 022111.17 & 1.52 & -051121.26 & 1.84 & $C$ & & $10.0 \pm 1.7$ & $13.4 \pm 6.5$ & $<4.2$ & $115.8 \pm 3.9$ & \\
\hline J0221.3-0255* & 022116.40 & 1.06 & -025549.21 & 1.75 & $M$ & $235.8 \pm 17.3$ & $147.3 \pm 14.8$ & $4.2 \pm 0.3$ & $2.5 \pm 0.3$ & $118.5 \pm 3.5$ & -0.61 \\
\hline & 022116.07 & 1.06 & -025553.70 & 1.75 & $C$ & & $88.5 \pm 9.0$ & $4.3 \pm 0.5$ & $3.2 \pm 0.5$ & $65.9 \pm 9.1$ & \\
\hline J0221.3-0555 & 022117.51 & 1.07 & -055601.42 & 1.76 & $S$ & $24.1 \pm 2.6$ & $24.1 \pm 2.6$ & $<3.2$ & $<2.5$ & $29.7 \pm 7.6$ & -0.80 \\
\hline J0221.3-0348 & 022117.81 & 1.16 & -034830.77 & 1.78 & $S$ & $6.2 \pm 1.0$ & $6.2 \pm 1.0$ & $<6.2$ & $<3.8$ & $61.0 \pm 8.9$ & -0.61 \\
\hline J0221.3-0227 & 022117.85 & 1.20 & -022713.18 & 1.77 & $S$ & $35.7 \pm 4.7$ & $35.7 \pm 4.7$ & $11.2 \pm 3.1$ & $<4.0$ & $97.2 \pm 5.1$ & -0.93 \\
\hline J0221.3-0457 & 022118.44 & 1.07 & -045723.15 & 1.75 & $S$ & $16.3 \pm 1.7$ & $16.3 \pm 1.7$ & $<2.7$ & $<2.4$ & $58.6 \pm 17.3$ & -0.15 \\
\hline J0221.3-0344 & 022119.38 & 1.07 & -034440.80 & 1.76 & $S$ & $15.8 \pm 1.8$ & $15.8 \pm 1.8$ & $3.5 \pm 1.9$ & $<2.9$ & $51.2 \pm 10.5$ & -0.21 \\
\hline J0221.3-0329 & 022119.91 & 1.12 & -032908.21 & 1.76 & $M$ & $16.2 \pm 1.5$ & $8.8 \pm 1.2$ & $<5.1$ & $<3.1$ & $98.3 \pm 5.9$ & -0.65 \\
\hline & 022119.93 & 1.08 & -032905.13 & 1.75 & $C$ & & $7.4 \pm 0.9$ & $<3.6$ & $<2.8$ & $102.3 \pm 13.8$ & \\
\hline J0221.3-0305* & 022121.16 & 1.16 & -030553.44 & 2.02 & $M$ & $64.9 \pm 6.5$ & $35.2 \pm 5.1$ & $19.3 \pm 5.2$ & $6.9 \pm 2.5$ & $163.5 \pm 4.5$ & -0.99 \\
\hline & 022119.76 & 1.21 & -030550.65 & 1.79 & $C$ & & $29.8 \pm 4.1$ & $11.7 \pm 3.2$ & $7.1 \pm 2.4$ & $91.2 \pm 10.6$ & \\
\hline J0221.4-0405 & 022121.28 & 1.34 & -040535.32 & 2.00 & $S$ & $4.9 \pm 0.9$ & $4.9 \pm 0.9$ & $<10.8$ & $<2.9$ & $139.9 \pm 2.4$ & $<-0.33$ \\
\hline J0221.4-0424 & 022123.94 & 1.13 & -042414.63 & 1.79 & $S$ & $6.8 \pm 1.1$ & $6.8 \pm 1.1$ & $<5.4$ & $\ldots$ & $\ldots$ & $<-0.56$ \\
\hline J0221.4-0352 & 022124.31 & 1.08 & -035240.04 & 1.76 & $S$ & $14.7 \pm 1.7$ & $14.7 \pm 1.7$ & $3.8 \pm 2.1$ & $<3.0$ & $60.8 \pm 9.1$ & -0.55 \\
\hline J0221.4-0347 & 022125.57 & 1.06 & -034720.93 & 1.75 & $S$ & $47.2 \pm 4.8$ & $47.2 \pm 4.8$ & $2.7 \pm 0.8$ & $<1.8$ & $43.2 \pm 6.4$ & -0.70 \\
\hline J0221.4-0202 & 022126.32 & 1.22 & -020235.13 & 1.81 & $M$ & $58.1 \pm 4.8$ & $16.8 \pm 2.7$ & $6.5 \pm 5.5$ & $<3.9$ & $126.8 \pm 5.6$ & -0.86 \\
\hline & 022127.03 & 1.13 & -020234.13 & 1.76 & $C$ & & $15.6 \pm 2.3$ & $<5.2$ & $<3.2$ & $84.8 \pm 8.4$ & \\
\hline & 022126.38 & 1.33 & -020235.02 & 1.84 & $C$ & & $13.7 \pm 2.5$ & $<8.9$ & $<4.1$ & $57.1 \pm 6.0$ & \\
\hline & 022127.10 & 1.22 & -020237.00 & 1.76 & $C$ & & $12.0 \pm 2.0$ & $<6.7$ & $<3.1$ & $88.8 \pm 5.7$ & \\
\hline J0221.5-0516 & 022127.26 & 1.08 & -051611.37 & 1.76 & $S$ & $12.8 \pm 1.5$ & $12.8 \pm 1.5$ & $<3.7$ & $<3.1$ & $58.4 \pm 15.8$ & -0.15 \\
\hline J0221.5-0504 & 022127.43 & 1.09 & -050402.04 & 1.78 & $S$ & $6.9 \pm 1.0$ & $6.9 \pm 1.0$ & $<4.7$ & $<3.9$ & $18.9 \pm 19.7$ & $<-0.57$ \\
\hline J0221.5-0434 & 022127.91 & 1.88 & -043411.41 & 1.78 & $S$ & $17.3 \pm 2.8$ & $17.3 \pm 2.8$ & $21.6 \pm 7.7$ & $<4.6$ & $86.1 \pm 2.8$ & -0.55 \\
\hline J0221.5-0509 & 022130.90 & 1.37 & -050929.44 & 1.88 & $S$ & $4.1 \pm 0.9$ & $4.1 \pm 0.9$ & $<8.6$ & $<7.0$ & $113.0 \pm 38.7$ & $<-0.22$ \\
\hline J0221.5-0556 & 022131.33 & 1.06 & -055626.03 & 1.75 & $M$ & $84.6 \pm 6.3$ & $49.3 \pm 5.1$ & $<2.2$ & $<1.8$ & $41.4 \pm 5.6$ & -0.56 \\
\hline & 022129.61 & 1.07 & -055623.83 & 1.75 & $C$ & & $35.3 \pm 3.8$ & $<2.8$ & $<2.4$ & $47.9 \pm 10.4$ & \\
\hline J0221.5-0423 & 022131.50 & 1.07 & -042347.52 & 1.75 & $S$ & $22.8 \pm 2.5$ & $22.8 \pm 2.5$ & $<2.8$ & $<2.6$ & $62.6 \pm 22.8$ & -0.59 \\
\hline J0221.5-0344 & 022132.68 & 1.09 & -034402.40 & 1.77 & $S$ & $7.5 \pm 1.0$ & $7.5 \pm 1.0$ & $<4.7$ & $<3.5$ & $47.9 \pm 13.8$ & 0.94 \\
\hline J0221.6-0409 & 022133.27 & 1.11 & -040904.02 & 1.76 & $M$ & $23.2 \pm 2.3$ & $15.8 \pm 2.0$ & $<4.9$ & $<3.0$ & $64.1 \pm 5.4$ & -0.55 \\
\hline & 022133.34 & 1.10 & -040900.42 & 1.77 & $C$ & & $7.4 \pm 1.2$ & $<5.0$ & $<2.9$ & $54.7 \pm 8.3$ & \\
\hline J0221.6-0236 & 022134.41 & 1.08 & -023616.60 & 1.77 & $S$ & $6.0 \pm 0.9$ & $6.0 \pm 0.9$ & $<4.4$ & $<4.0$ & $-1.0 \pm 55.9$ & $<-0.48$ \\
\hline J0221.6-0518 & 022135.61 & 1.11 & -051822.56 & 1.82 & $M$ & $24.7 \pm 2.4$ & $16.8 \pm 2.2$ & $10.3 \pm 3.2$ & $<3.2$ & $30.8 \pm 3.2$ & -0.59 \\
\hline & 022136.02 & 1.09 & -051817.40 & 1.78 & $C$ & & $7.8 \pm 1.1$ & $<4.8$ & $<4.1$ & $1.5 \pm 22.8$ & \\
\hline J0221.7-0509 & 022141.38 & 1.08 & -050943.50 & 1.77 & $S$ & $9.2 \pm 1.1$ & $9.2 \pm 1.1$ & $<4.0$ & $<3.5$ & $12.8 \pm 19.3$ & -0.31 \\
\hline J0221.7-0451* & 022142.06 & 1.06 & -045112.38 & 1.75 & $S$ & $198.3 \pm 19.9$ & $198.3 \pm 19.9$ & $10.6 \pm 0.3$ & $2.0 \pm 0.3$ & $166.3 \pm 0.4$ & -0.88 \\
\hline J0221.7-0413* & 022143.06 & 1.06 & -041345.57 & 1.75 & $S$ & $1269.7 \pm 127.0$ & $1269.7 \pm 127.0$ & $7.5 \pm 0.1$ & $2.2 \pm 0.1$ & $135.0 \pm 0.2$ & -0.59 \\
\hline
\end{tabular}


C. Tasse et al.: The low-frequency counterpart of the XMM large scale structure survey, Online Material $p 13$

Table A.1. continued.

\begin{tabular}{|c|c|c|c|c|c|c|c|c|c|c|c|}
\hline Name & $\begin{array}{c}\text { RA } \\
\text { J2000 }\end{array}$ & $\begin{array}{c}\sigma_{\alpha} \\
\operatorname{arcsec}\end{array}$ & $\begin{array}{c}\text { Dec } \\
\text { J2000 }\end{array}$ & $\begin{array}{c}\sigma_{\delta} \\
\operatorname{arcsec}\end{array}$ & Type & $\begin{array}{l}S_{\text {int }} \\
\text { mJy }\end{array}$ & $\begin{array}{c}S_{\text {comp }} \\
\text { mJy }\end{array}$ & $\begin{array}{c}\text { Major axis } \\
\text { arcsec }\end{array}$ & $\begin{array}{c}\text { Minor axis } \\
\text { arcsec }\end{array}$ & $\begin{array}{c}\text { Position } \\
\text { Angle }\end{array}$ & $\begin{array}{c}\text { Spectral } \\
\text { index } \alpha_{325}^{1400}\end{array}$ \\
\hline J0221.7-0249 & 022143.30 & 1.06 & -024929.82 & 1.75 & $\bar{S}$ & $26.9 \pm 2.8$ & $26.9 \pm 2.8$ & $<2.6$ & $<2.2$ & $53.6 \pm 7.5$ & -0.52 \\
\hline J0221.7-0404 & 022143.73 & 1.17 & -040427.45 & 1.99 & $S$ & $7.0 \pm 1.5$ & $7.0 \pm 1.5$ & $<9.0$ & $<6.0$ & $174.8 \pm 17.7$ & $<-0.58$ \\
\hline \multirow[t]{2}{*}{ J0221.7-0357 } & 022144.28 & 1.09 & -035750.62 & 1.76 & $M$ & $28.3 \pm 2.6$ & $15.9 \pm 2.0$ & $3.8 \pm 2.7$ & $<3.7$ & $59.1 \pm 17.7$ & -0.80 \\
\hline & 022145.44 & 1.09 & -035741.75 & 1.77 & $C$ & & $12.4 \pm 1.7$ & $<4.6$ & $<3.6$ & $51.7 \pm 13.1$ & \\
\hline \multirow[t]{2}{*}{ J0221.8-0329 } & 022145.60 & 1.07 & -03 2929.03 & 1.76 & $M$ & $17.1 \pm 1.5$ & $9.1 \pm 1.1$ & $<3.4$ & $<2.7$ & $44.0 \pm 12.9$ & -1.25 \\
\hline & 022145.63 & 1.12 & -032932.19 & 1.76 & $C$ & & $8.0 \pm 1.1$ & $<5.1$ & $<3.0$ & $72.4 \pm 5.6$ & \\
\hline J0221.8-0308 & 022146.33 & 1.24 & -030835.06 & 1.88 & $S$ & $3.6 \pm 0.8$ & $3.6 \pm 0.8$ & $<7.4$ & $\ldots$ & $\ldots$ & $<-0.13$ \\
\hline J0221.8-0456 & 022146.85 & 1.09 & -045646.34 & 1.78 & $S$ & $7.9 \pm 1.1$ & $7.9 \pm 1.1$ & $<4.6$ & $<4.3$ & $18.9 \pm 55.5$ & -0.64 \\
\hline J0221.8-0326 & 022147.76 & 1.08 & -032639.03 & 1.76 & $S$ & $11.1 \pm 1.4$ & $11.1 \pm 1.4$ & $<3.9$ & $<3.6$ & $87.5 \pm 47.5$ & -0.68 \\
\hline J0221.8-0257 & 022148.00 & 1.18 & -025716.13 & 1.78 & $S$ & $4.4 \pm 0.8$ & $4.4 \pm 0.8$ & $<6.4$ & $<4.5$ & $75.2 \pm 17.5$ & $<-0.26$ \\
\hline J0221.9-0505 & 022151.43 & 1.11 & -050538.19 & 1.77 & $S$ & $7.9 \pm 1.0$ & $7.9 \pm 1.0$ & $4.5 \pm 3.4$ & $<3.3$ & $59.4 \pm 6.6$ & -0.40 \\
\hline J0221.9-0427 & 022151.92 & 1.08 & -042728.81 & 1.86 & $S$ & $6.0 \pm 1.0$ & $6.0 \pm 1.0$ & $<6.9$ & $<4.0$ & $0.5 \pm 8.6$ & -0.54 \\
\hline \multirow[t]{2}{*}{ J0221.9-0205 } & 022152.78 & 1.11 & -020504.11 & 1.77 & $M$ & $72.6 \pm 6.5$ & $37.9 \pm 4.8$ & $7.0 \pm 2.4$ & $<3.6$ & $54.5 \pm 7.3$ & -0.68 \\
\hline & 022153.17 & 1.12 & -020509.38 & 1.76 & $C$ & & $34.7 \pm 4.5$ & $6.6 \pm 2.5$ & $<3.9$ & $96.5 \pm 10.4$ & \\
\hline J0221.9-0318 & 022156.49 & 1.21 & -031814.84 & 1.79 & $S$ & $4.8 \pm 0.9$ & $4.8 \pm 0.9$ & $<6.7$ & $<5.0$ & $103.9 \pm 20.9$ & -0.39 \\
\hline J0221.9-0407 & 022156.49 & 1.10 & -040758.48 & 1.77 & $S$ & $10.1 \pm 1.4$ & $10.1 \pm 1.4$ & $<4.8$ & $<3.9$ & $44.4 \pm 16.0$ & -0.83 \\
\hline J0222.0-0443 & 022159.78 & 1.12 & -044327.62 & 1.78 & $S$ & $5.7 \pm 0.9$ & $5.7 \pm 0.9$ & $<5.1$ & $<4.6$ & $59.9 \pm 49.1$ & $<-0.44$ \\
\hline J0222.0-0339 & 022200.39 & 1.06 & -033935.94 & 1.75 & $S$ & $50.0 \pm 5.1$ & $50.0 \pm 5.1$ & $4.4 \pm 0.6$ & $<1.9$ & $66.0 \pm 5.2$ & -0.62 \\
\hline $\mathrm{J} 0222.0-0550$ & 022200.83 & 1.08 & -055100.19 & 1.79 & $S$ & $8.7 \pm 1.3$ & $8.7 \pm 1.3$ & $<5.2$ & $<3.7$ & $1.4 \pm 12.3$ & -0.42 \\
\hline \multirow[t]{8}{*}{ J0222.0-0240* } & 022201.29 & 1.06 & -024014.93 & 1.75 & $M$ & $473.7 \pm 27.5$ & $240.8 \pm 24.1$ & $5.1 \pm 0.2$ & $2.1 \pm 0.3$ & $140.3 \pm 1.3$ & -0.76 \\
\hline & 022159.98 & 1.06 & -024000.46 & 1.75 & $C$ & & $108.8 \pm 11.0$ & $7.0 \pm 0.5$ & $2.9 \pm 0.6$ & $118.3 \pm 2.2$ & \\
\hline & 022159.55 & 1.06 & -023955.42 & 1.75 & $C$ & & $54.2 \pm 5.5$ & $<2.0$ & $<1.9$ & $50.6 \pm 51.2$ & \\
\hline & 022200.87 & 1.19 & -024010.19 & 1.79 & $C$ & & $27.1 \pm 3.2$ & $11.5 \pm 3.7$ & $<1.8$ & $54.4 \pm 0.8$ & \\
\hline & 022200.74 & 1.08 & -024007.82 & 1.76 & $C$ & & $17.8 \pm 2.1$ & $<4.0$ & $<2.7$ & $66.0 \pm 5.7$ & \\
\hline & 022200.97 & 1.07 & -024020.30 & 1.75 & $C$ & & $11.5 \pm 1.4$ & $<2.8$ & $<2.4$ & $41.7 \pm 20.7$ & \\
\hline & 022200.72 & 1.09 & -024019.33 & 1.89 & $C$ & & $7.9 \pm 1.2$ & $<7.6$ & $<3.2$ & $163.4 \pm 3.9$ & \\
\hline & 022159.17 & 1.27 & -024001.11 & 2.07 & $C$ & & $5.6 \pm 1.3$ & $<10.4$ & $<6.1$ & $153.0 \pm 13.0$ & \\
\hline J0222.0-0337 & 022202.88 & 1.11 & -033716.13 & 1.84 & $S$ & $3.8 \pm 0.7$ & $3.8 \pm 0.7$ & $<6.9$ & $<3.6$ & $25.3 \pm 8.3$ & $<-0.16$ \\
\hline J0222.1-0448 & 022203.93 & 1.08 & -044835.93 & 1.77 & $S$ & $5.7 \pm 0.8$ & $5.7 \pm 0.8$ & $<4.1$ & $<3.5$ & $14.5 \pm 30.3$ & $<-0.44$ \\
\hline \multirow[t]{2}{*}{$\mathrm{J} 0222.1-0338$} & 022204.80 & 1.06 & -033816.22 & 1.75 & $M$ & $96.4 \pm 9.2$ & $91.1 \pm 9.1$ & $2.5 \pm 0.4$ & $1.1 \pm 0.8$ & $16.9 \pm 7.4$ & -0.49 \\
\hline & 022204.40 & 1.08 & -033820.99 & 1.76 & $C$ & & $5.3 \pm 0.8$ & $<4.3$ & $<3.2$ & $47.9 \pm 15.2$ & \\
\hline J0222.1-0320 & 022206.21 & 1.23 & -03 2039.87 & 1.84 & $S$ & $19.0 \pm 2.9$ & $19.0 \pm 2.9$ & $10.5 \pm 3.6$ & $9.2 \pm 3.3$ & $77.9 \pm 48.9$ & -0.30 \\
\hline J0222.1-0334 & 022206.29 & 1.06 & -033420.23 & 1.75 & $S$ & $23.8 \pm 2.5$ & $23.8 \pm 2.5$ & $<2.6$ & $<2.4$ & $34.9 \pm 16.4$ & -0.36 \\
\hline J0222.1-0504 & 022206.73 & 1.12 & -050418.36 & 1.90 & $S$ & $3.3 \pm 0.7$ & $3.3 \pm 0.7$ & $<7.6$ & $<5.1$ & $173.3 \pm 17.3$ & $<-0.07$ \\
\hline J0222.1-0232 & 022207.70 & 1.06 & -023301.09 & 1.77 & $S$ & $7.6 \pm 0.8$ & $7.6 \pm 0.8$ & $<4.3$ & $<1.0$ & $179.4 \pm 0.7$ & -0.04 \\
\hline J0222.1-0428 & 022207.91 & 1.29 & -042809.48 & 1.84 & $S$ & $4.7 \pm 0.9$ & $4.7 \pm 0.9$ & $<8.5$ & $<4.8$ & $124.9 \pm 9.2$ & 0.64 \\
\hline J0222.2-0452 & 022211.59 & 1.16 & -045221.91 & 1.84 & $S$ & $3.8 \pm 0.7$ & $3.8 \pm 0.7$ & $<6.7$ & $<5.7$ & $156.9 \pm 42.7$ & $<-0.17$ \\
\hline J0222.2-0549 & 022211.82 & 1.06 & -054905.85 & 1.75 & $S$ & $83.8 \pm 8.5$ & $83.8 \pm 8.5$ & $<1.6$ & $<1.5$ & $38.3 \pm 8.6$ & -0.97 \\
\hline J0222.2-0222 & 022211.91 & 1.12 & -022217.50 & 1.77 & $S$ & $8.5 \pm 1.3$ & $8.5 \pm 1.3$ & $<5.2$ & $<4.1$ & $71.7 \pm 20.3$ & -0.70 \\
\hline J0222.2-0428 & 022213.03 & 1.10 & -042833.86 & 1.77 & $S$ & $7.9 \pm 1.1$ & $7.9 \pm 1.1$ & $<4.6$ & $<4.1$ & $132.3 \pm 35.6$ & -0.66 \\
\hline J0222.2-0306 & 022214.68 & 1.09 & -030651.06 & 1.84 & $S$ & $4.2 \pm 0.7$ & $4.2 \pm 0.7$ & $<6.7$ & $<3.9$ & $11.7 \pm 10.0$ & $<-0.23$ \\
\hline J0222.3-0249 & 022218.50 & 1.10 & -024935.83 & 1.76 & $S$ & $8.5 \pm 1.2$ & $8.5 \pm 1.2$ & $<4.5$ & $<3.3$ & $106.7 \pm 13.6$ & -0.42 \\
\hline $\mathrm{J} 0222.3-0240$ & 022218.63 & 1.16 & -024031.81 & 1.80 & $S$ & $7.2 \pm 1.2$ & $7.2 \pm 1.2$ & $<6.3$ & $<5.1$ & $128.3 \pm 26.8$ & -0.17 \\
\hline \multirow[t]{2}{*}{ J0222.3-0242* } & 022220.70 & 1.06 & -024216.47 & 1.75 & $M$ & $201.4 \pm 14.9$ & $127.8 \pm 12.9$ & $4.7 \pm 0.3$ & $3.2 \pm 0.4$ & $41.7 \pm 4.7$ & -1.13 \\
\hline & 022220.81 & 1.06 & -024231.02 & 1.75 & $C$ & & $73.6 \pm 7.5$ & $4.8 \pm 0.6$ & $3.8 \pm 0.6$ & $74.2 \pm 12.6$ & \\
\hline J0222.4-0217 & 022220.90 & 1.23 & -021740.96 & 1.85 & $S$ & $18.0 \pm 2.7$ & $18.0 \pm 2.7$ & $11.3 \pm 4.6$ & $<4.3$ & $133.1 \pm 5.2$ & -0.67 \\
\hline J0222.4-0231 & 022221.15 & 1.24 & -023132.30 & 1.99 & $S$ & $5.6 \pm 1.3$ & $5.6 \pm 1.3$ & $<9.5$ & $<5.8$ & $152.2 \pm 14.5$ & $<-0.42$ \\
\hline J0222.4-0453 & 022222.85 & 1.10 & -045326.48 & 1.76 & $M$ & $9.8 \pm 1.0$ & $6.6 \pm 0.9$ & $<4.6$ & $<3.2$ & $78.2 \pm 9.4$ & -0.70 \\
\hline & 022222.80 & 1.14 & -045323.63 & 1.77 & $C$ & & $3.2 \pm 0.5$ & $<5.9$ & $<3.2$ & $58.4 \pm 8.0$ & \\
\hline J0222.4-0349 & 022224.90 & 1.11 & -034954.79 & 1.76 & $M$ & $9.7 \pm 1.0$ & $5.7 \pm 0.8$ & $<5.0$ & $<3.3$ & $98.0 \pm 8.3$ & -0.60 \\
\hline & 022224.89 & 1.10 & -034951.64 & 1.76 & $C$ & & $4.0 \pm 0.6$ & $<4.5$ & $<3.1$ & $98.8 \pm 11.8$ & \\
\hline J0222.5-0407 & 022227.74 & 1.20 & -040718.80 & 1.85 & $M$ & $7.8 \pm 1.0$ & $4.3 \pm 0.8$ & $<7.9$ & $<4.1$ & $42.9 \pm 7.3$ & $<-0.66$ \\
\hline & 022227.46 & 1.16 & -040718.46 & 1.79 & $C$ & & $3.5 \pm 0.6$ & $<6.6$ & $<3.5$ & $51.6 \pm 8.3$ & \\
\hline J0222.5-0431 & 022228.29 & 1.08 & -043123.48 & 1.76 & $M$ & $15.4 \pm 1.5$ & $10.4 \pm 1.2$ & $<3.7$ & $<3.3$ & $97.4 \pm 19.0$ & -0.58 \\
\hline & 022228.89 & 1.12 & -043139.73 & 1.79 & $C$ & & $5.0 \pm 0.8$ & $<5.3$ & $<4.7$ & $138.1 \pm 42.6$ & \\
\hline J0222.5-0307* & 022228.73 & 1.06 & -030744.72 & 1.75 & $S$ & $66.6 \pm 6.7$ & $66.6 \pm 6.7$ & $2.0 \pm 0.6$ & $<1.5$ & $46.1 \pm 13.2$ & -0.74 \\
\hline J0222.5-0333 & 022229.57 & 1.14 & -033316.46 & 1.77 & $S$ & $4.0 \pm 0.7$ & $4.0 \pm 0.7$ & $<5.7$ & $<4.1$ & $111.7 \pm 17.4$ & $<-0.20$ \\
\hline J0222.5-0352 & 022229.87 & 1.12 & -035238.60 & 1.82 & $S$ & $4.2 \pm 0.7$ & $4.2 \pm 0.7$ & $<6.1$ & $<5.0$ & $3.7 \pm 30.5$ & -0.31 \\
\hline J0222.5-0447 & 022230.17 & 1.07 & -044705.45 & 1.75 & $M$ & $53.8 \pm 4.0$ & $28.7 \pm 3.0$ & $4.5 \pm 0.9$ & $<2.3$ & $71.3 \pm 6.9$ & -0.89 \\
\hline & 022230.96 & 1.07 & -044707.56 & 1.75 & $C$ & & $25.1 \pm 2.6$ & $4.3 \pm 1.1$ & $<2.5$ & $73.9 \pm 10.1$ & \\
\hline J0222.6-0414 & 022236.71 & 1.09 & -041458.01 & 1.81 & $S$ & $3.8 \pm 0.6$ & $3.8 \pm 0.6$ & $<5.9$ & $<3.6$ & $159.0 \pm 10.4$ & $<-0.17$ \\
\hline J0222.7-0334 & 022242.29 & 1.14 & -033418.23 & 1.83 & $S$ & $4.8 \pm 0.9$ & $4.8 \pm 0.9$ & $<6.5$ & $<5.1$ & $152.1 \pm 25.6$ & 0.23 \\
\hline J0222.7-0402 & 022244.35 & 1.20 & -040235.29 & 1.84 & $S$ & $3.4 \pm 0.7$ & $3.4 \pm 0.7$ & $<7.1$ & $<5.8$ & $45.2 \pm 34.3$ & -0.28 \\
\hline J0222.8-0222 & 022245.56 & 1.06 & -022222.74 & 1.75 & $S$ & $31.9 \pm 3.4$ & $31.9 \pm 3.4$ & $<2.5$ & $<2.2$ & $81.0 \pm 10.3$ & -0.96 \\
\hline J0222.8-0512 & 022245.82 & 1.09 & -051217.18 & 1.92 & $S$ & $4.4 \pm 0.9$ & $4.4 \pm 0.9$ & $<7.9$ & $<4.3$ & $178.7 \pm 10.3$ & $<-0.26$ \\
\hline J0222.8-0303 & 022246.16 & 1.06 & -030354.43 & 1.75 & $S$ & $41.1 \pm 4.2$ & $41.1 \pm 4.2$ & $<2.0$ & $<1.8$ & $58.7 \pm 9.3$ & -0.50 \\
\hline $\mathrm{J} 0222.8-0348$ & 022247.61 & 1.06 & -034817.05 & 1.75 & $S$ & $32.6 \pm 3.3$ & $32.6 \pm 3.3$ & $<2.0$ & $<1.8$ & $37.1 \pm 9.2$ & -0.63 \\
\hline J0222.8-0344 & 022248.31 & 1.68 & -034435.72 & 1.97 & $S$ & $6.8 \pm 1.4$ & $6.8 \pm 1.4$ & $10.5 \pm 9.9$ & $<4.2$ & $56.0 \pm 4.0$ & $<-0.56$ \\
\hline J0222.8-0412 & 022248.76 & 1.08 & -041215.77 & 1.76 & $S$ & $8.8 \pm 1.0$ & $8.8 \pm 1.0$ & $<3.7$ & $<3.1$ & $49.3 \pm 14.5$ & -0.87 \\
\hline J0222.8-0311 & 022250.04 & 1.10 & -031151.61 & 1.80 & $S$ & $12.3 \pm 1.7$ & $12.3 \pm 1.7$ & $6.6 \pm 2.9$ & $<4.2$ & $19.9 \pm 11.4$ & -0.78 \\
\hline J0222.9-0432 & 022251.15 & 1.10 & -043214.25 & 1.79 & $S$ & $5.6 \pm 0.9$ & $5.6 \pm 0.9$ & $<5.4$ & $<4.2$ & $28.8 \pm 19.9$ & -0.19 \\
\hline J0222.9-0416 & 022252.06 & 1.12 & -041646.38 & 1.77 & $M$ & $9.7 \pm 1.0$ & $6.0 \pm 0.8$ & $<5.3$ & $<3.6$ & $57.1 \pm 9.1$ & -0.69 \\
\hline & 022252.13 & 1.08 & -041648.97 & 1.78 & $C$ & & $3.7 \pm 0.6$ & $<4.8$ & $<3.4$ & $18.5 \pm 13.9$ & \\
\hline J0222.9-0525 & 022254.08 & 1.26 & -052531.75 & 1.85 & $S$ & $7.0 \pm 1.5$ & $7.0 \pm 1.5$ & $<8.3$ & $<5.1$ & $49.8 \pm 14.0$ & $<-0.58$ \\
\hline
\end{tabular}


C. Tasse et al.: The low-frequency counterpart of the XMM large scale structure survey, Online Material p 14

Table A.1. continued.

\begin{tabular}{|c|c|c|c|c|c|c|c|c|c|c|c|}
\hline Name & $\begin{array}{c}\text { RA } \\
\text { J2000 }\end{array}$ & $\begin{array}{c}\sigma_{\alpha} \\
\operatorname{arcsec}\end{array}$ & $\begin{array}{c}\text { Dec } \\
\text { J2000 }\end{array}$ & $\begin{array}{c}\sigma_{\delta} \\
\operatorname{arcsec}\end{array}$ & Type & $\begin{array}{l}S_{\text {int }} \\
\text { mJy }\end{array}$ & $\begin{array}{c}S_{\text {comp }} \\
\text { mJy }\end{array}$ & $\begin{array}{c}\text { Major axis } \\
\text { arcsec }\end{array}$ & $\begin{array}{c}\text { Minor axis } \\
\text { arcsec }\end{array}$ & $\begin{array}{c}\text { Position } \\
\text { Angle }\end{array}$ & $\begin{array}{c}\text { Spectral } \\
\text { index } \alpha_{325}^{1400}\end{array}$ \\
\hline \multirow[t]{2}{*}{ J0222.9-0311* } & 022254.60 & 1.07 & -031129.87 & 1.75 & $\bar{M}$ & $50.3 \pm 4.9$ & $46.5 \pm 4.8$ & $6.4 \pm 0.8$ & $4.0 \pm 0.8$ & $112.6 \pm 6.0$ & -1.35 \\
\hline & 022253.82 & 1.37 & -031128.37 & 1.93 & $C$ & & $3.8 \pm 0.9$ & $<9.1$ & $<7.3$ & $51.8 \pm 36.3$ & \\
\hline J0222.9-0414 & 022254.62 & 1.10 & -041404.77 & 1.78 & $S$ & $17.5 \pm 2.2$ & $17.5 \pm 2.2$ & $7.0 \pm 2.2$ & $4.6 \pm 2.1$ & $39.7 \pm 16.7$ & -0.26 \\
\hline J0222.9-0309 & 022254.62 & 1.14 & -030943.21 & 1.81 & $S$ & $6.5 \pm 1.0$ & $6.5 \pm 1.0$ & $<6.5$ & $<4.2$ & $40.8 \pm 9.8$ & $<-0.53$ \\
\hline \multirow[t]{4}{*}{ J0222.9-0518* } & 022255.76 & 1.06 & -051817.15 & 1.75 & $M$ & $577.3 \pm 54.4$ & $543.2 \pm 54.4$ & $3.5 \pm 0.1$ & $2.9 \pm 0.1$ & $49.6 \pm 3.7$ & -0.53 \\
\hline & 022256.15 & 1.09 & -051824.05 & 1.83 & $C$ & & $12.9 \pm 1.8$ & $<6.6$ & $<2.7$ & $22.1 \pm 2.9$ & \\
\hline & 022256.01 & 1.06 & -051822.35 & 1.76 & $C$ & & $11.6 \pm 1.4$ & $<3.5$ & $<1.6$ & $8.4 \pm 2.6$ & \\
\hline & 022256.13 & 1.22 & -051810.01 & 1.79 & $C$ & & $9.6 \pm 1.6$ & $<7.3$ & $<3.6$ & $62.1 \pm 6.1$ & \\
\hline \multirow[t]{5}{*}{ J0223.0-0242* } & 022256.07 & 1.06 & -024304.02 & 1.75 & $M$ & $1489.9 \pm 94.2$ & $776.3 \pm 77.7$ & $6.4 \pm 0.1$ & $3.0 \pm 0.1$ & $93.1 \pm 0.6$ & -0.84 \\
\hline & 022258.65 & 1.06 & -024253.31 & 1.75 & $C$ & & $515.7 \pm 51.6$ & $5.4 \pm 0.1$ & $2.6 \pm 0.2$ & $80.0 \pm 1.0$ & \\
\hline & 022256.65 & 1.07 & -024303.89 & 1.82 & $C$ & & $83.6 \pm 9.1$ & $16.1 \pm 2.7$ & $<1.8$ & $16.9 \pm 0.6$ & \\
\hline & 022257.78 & 1.08 & -024256.14 & 1.76 & $C$ & & $63.3 \pm 7.2$ & $7.3 \pm 1.6$ & $4.9 \pm 1.5$ & $48.4 \pm 11.9$ & \\
\hline & 022258.14 & 1.07 & -024252.44 & 2.04 & $C$ & & $50.9 \pm 6.4$ & $18.0 \pm 5.3$ & $<2.5$ & $175.0 \pm 1.0$ & \\
\hline \multirow[t]{3}{*}{ J0222.9-0424* } & 022256.37 & 1.06 & -042449.55 & 1.75 & $M$ & $70.0 \pm 5.0$ & $41.4 \pm 4.2$ & $3.0 \pm 0.7$ & $<1.8$ & $24.7 \pm 5.0$ & -0.98 \\
\hline & 022256.66 & 1.16 & -042452.40 & 1.76 & $C$ & & $19.7 \pm 2.4$ & $11.1 \pm 2.8$ & $<2.8$ & $111.5 \pm 2.1$ & \\
\hline & 022257.23 & 1.10 & -042452.44 & 1.77 & $C$ & & $8.9 \pm 1.2$ & $4.1 \pm 2.9$ & $<4.0$ & $131.1 \pm 21.7$ & \\
\hline J0223.0-0235 & 022257.91 & 1.24 & -023537.05 & 1.97 & $S$ & $8.6 \pm 1.8$ & $8.6 \pm 1.8$ & $<9.4$ & $<5.4$ & $32.6 \pm 11.1$ & $<-0.72$ \\
\hline J0223.0-0407 & 022258.05 & 1.19 & -040702.54 & 1.86 & $S$ & $4.2 \pm 0.8$ & $4.2 \pm 0.8$ & $<6.9$ & $\ldots$ & $\ldots$ & -0.24 \\
\hline \multirow[t]{2}{*}{ J0223.0-0504 } & 022258.22 & 1.07 & -050419.64 & 1.77 & $M$ & $25.3 \pm 2.5$ & $19.1 \pm 2.3$ & $5.6 \pm 2.0$ & $<3.3$ & $178.8 \pm 9.0$ & -0.80 \\
\hline & 022258.07 & 1.29 & -050428.94 & 1.80 & $C$ & & $6.2 \pm 1.1$ & $<8.1$ & $<4.5$ & $65.3 \pm 7.7$ & \\
\hline J0223.0-0232 & 022258.87 & 1.07 & -023301.34 & 1.76 & $S$ & $16.9 \pm 2.1$ & $16.9 \pm 2.1$ & $<3.8$ & $<3.2$ & $15.3 \pm 16.5$ & -0.68 \\
\hline \multirow[t]{2}{*}{ J0223.0-0354 } & 022259.79 & 1.10 & -035405.55 & 1.77 & $M$ & $37.9 \pm 3.7$ & $24.5 \pm 2.9$ & $8.4 \pm 1.8$ & $7.9 \pm 1.8$ & $94.7 \pm 70.8$ & -0.78 \\
\hline & 022300.16 & 1.25 & -035339.84 & 1.86 & $C$ & & $13.4 \pm 2.2$ & $9.6 \pm 4.1$ & $8.3 \pm 3.8$ & $56.4 \pm 58.7$ & \\
\hline J0223.0-0409 & 022301.29 & 1.10 & -040936.13 & 1.77 & $S$ & $6.6 \pm 0.9$ & $6.6 \pm 0.9$ & $<4.7$ & $<4.1$ & $69.5 \pm 27.5$ & -0.20 \\
\hline J0223.0-0428 & 022302.43 & 1.13 & -042848.96 & 1.80 & $S$ & $4.6 \pm 0.8$ & $4.6 \pm 0.8$ & $<5.5$ & $\ldots$ & $\ldots$ & $<-0.30$ \\
\hline J0223.1-0444 & 022306.66 & 1.07 & -044444.74 & 1.76 & $S$ & $8.9 \pm 1.1$ & $8.9 \pm 1.1$ & $<3.9$ & $<3.3$ & $169.5 \pm 17.8$ & -0.07 \\
\hline J0223.2-0423 & 022310.14 & 1.06 & -042306.92 & 1.75 & $S$ & $44.5 \pm 4.5$ & $44.5 \pm 4.5$ & $2.2 \pm 0.7$ & $1.3 \pm 1.1$ & $13.2 \pm 24.5$ & -0.13 \\
\hline J0223.2-0205* & 022312.99 & 1.06 & -020510.41 & 1.75 & $S$ & $156.5 \pm 15.7$ & $156.5 \pm 15.7$ & $1.5 \pm 0.6$ & $<1.3$ & $121.8 \pm 13.1$ & -0.09 \\
\hline J0223.3-0207 & 022315.50 & 1.12 & -020714.72 & 1.82 & $S$ & $9.4 \pm 1.7$ & $9.4 \pm 1.7$ & $<6.2$ & $<5.0$ & $21.2 \pm 28.1$ & -0.00 \\
\hline J0223.3-0458 & 022316.61 & 1.09 & -045844.72 & 1.79 & $S$ & $5.7 \pm 0.8$ & $5.7 \pm 0.8$ & $<5.1$ & $<4.2$ & $166.8 \pm 24.0$ & $<-0.44$ \\
\hline J0223.3-0444 & 022317.00 & 1.37 & -044450.89 & 1.90 & $S$ & $3.5 \pm 0.8$ & $3.5 \pm 0.8$ & $<8.8$ & $<7.2$ & $121.8 \pm 39.8$ & $<-0.10$ \\
\hline J0223.3-0445 & 022319.15 & 1.28 & -044549.22 & 1.87 & $S$ & $3.9 \pm 0.8$ & $3.9 \pm 0.8$ & $<8.4$ & $<5.8$ & $131.0 \pm 15.5$ & $<-0.17$ \\
\hline J0223.3-0318 & 022320.72 & 1.06 & -031825.44 & 1.75 & $M$ & $26.2 \pm 2.4$ & $21.2 \pm 2.2$ & $<2.4$ & $<2.0$ & $46.3 \pm 6.3$ & -0.15 \\
\hline & 022318.40 & 1.12 & -031850.82 & 1.80 & $C$ & & $5.0 \pm 0.8$ & $<5.6$ & $<4.7$ & $33.0 \pm 27.8$ & \\
\hline J0223.4-0528 & 022321.63 & 1.06 & -052804.66 & 1.83 & $M$ & $42.7 \pm 3.8$ & $28.9 \pm 2.9$ & $12.3 \pm 2.8$ & $<0.3$ & $179.6 \pm 0.0$ & $<-1.82$ \\
\hline & 022321.85 & 1.08 & -052807.58 & 2.48 & $C$ & & $13.8 \pm 2.4$ & $17.8 \pm 9.0$ & $<3.7$ & $2.7 \pm 2.1$ & \\
\hline J0223.4-0222 & 022322.67 & 1.09 & -022220.33 & 1.77 & $S$ & $7.0 \pm 1.0$ & $7.0 \pm 1.0$ & $<4.2$ & $<4.0$ & $-1.0 \pm 82.5$ & $<-0.58$ \\
\hline J0223.4-0541 & 022322.75 & 1.10 & -054145.66 & 1.80 & $S$ & $5.1 \pm 0.9$ & $5.1 \pm 0.9$ & $<5.8$ & $<3.8$ & $28.0 \pm 12.8$ & -0.45 \\
\hline J0223.4-0427 & 022324.95 & 1.07 & -042737.76 & 1.76 & $M$ & $46.0 \pm 3.4$ & $19.8 \pm 2.2$ & $6.3 \pm 1.4$ & $<2.9$ & $32.8 \pm 6.8$ & -0.59 \\
\hline & 022325.52 & 1.08 & -042709.23 & 1.77 & $C$ & & $15.9 \pm 1.9$ & $6.9 \pm 1.8$ & $3.0 \pm 2.1$ & $26.2 \pm 9.4$ & \\
\hline & 022325.37 & 1.37 & -042722.41 & 2.10 & $C$ & & $10.2 \pm 1.7$ & $15.1 \pm 7.6$ & $<3.9$ & $36.3 \pm 2.9$ & \\
\hline J0223.5-0540 & 022327.42 & 1.07 & -054044.72 & 1.76 & $S$ & $15.2 \pm 1.7$ & $15.2 \pm 1.7$ & $<3.3$ & $<2.7$ & $39.3 \pm 9.9$ & -0.70 \\
\hline J0223.5-0303 & 022329.38 & 1.18 & -030357.49 & 1.84 & $S$ & $4.0 \pm 0.8$ & $4.0 \pm 0.8$ & $<7.7$ & $<3.6$ & $42.0 \pm 6.6$ & $<-0.20$ \\
\hline J0223.5-0401 & 022332.34 & 1.16 & -040145.68 & 1.79 & $S$ & $3.5 \pm 0.6$ & $3.5 \pm 0.6$ & $<6.1$ & $<4.7$ & $114.6 \pm 24.0$ & -0.21 \\
\hline J0223.5-0514 & 022332.41 & 1.10 & -051437.41 & 1.76 & $S$ & $11.3 \pm 1.4$ & $11.3 \pm 1.4$ & $4.7 \pm 2.8$ & $<3.0$ & $52.5 \pm 5.2$ & -0.86 \\
\hline J0223.6-0301* & 022333.15 & 1.06 & -030123.95 & 1.75 & $M$ & $200.8 \pm 18.4$ & $183.6 \pm 18.4$ & $1.8 \pm 0.3$ & $1.2 \pm 0.4$ & $147.2 \pm 10.6$ & -1.09 \\
\hline & 022332.77 & 1.06 & -030128.07 & 1.76 & $C$ & & $10.4 \pm 1.2$ & $<3.2$ & $<2.1$ & $176.2 \pm 5.2$ & \\
\hline & 022333.57 & 1.07 & -030127.37 & 1.76 & $C$ & & $6.9 \pm 0.9$ & $<4.0$ & $<2.4$ & $146.6 \pm 5.7$ & \\
\hline J0223.6-0458 & 022334.06 & 1.32 & -045836.72 & 1.80 & $S$ & $66.4 \pm 12.9^{*}$ & $32.6 \pm 4.4$ & $17.5 \pm 4.1$ & $8.2 \pm 2.3$ & $103.3 \pm 5.5$ & -0.38 \\
\hline J0223.6-0232* & 022334.68 & 1.06 & -023224.03 & 1.76 & $M$ & $172.8 \pm 12.4$ & $89.6 \pm 9.2$ & $11.4 \pm 0.8$ & $2.1 \pm 0.8$ & $14.7 \pm 1.1$ & -0.91 \\
\hline & 022334.80 & 1.06 & -023219.22 & 1.75 & $C$ & & $83.2 \pm 8.4$ & $1.6 \pm 0.7$ & $<1.4$ & $129.9 \pm 4.1$ & \\
\hline J0223.6-0348 & 022335.75 & 1.20 & -034847.68 & 1.87 & $S$ & $3.1 \pm 0.7$ & $3.1 \pm 0.7$ & $<7.4$ & $<6.3$ & $29.3 \pm 49.9$ & $<-0.02$ \\
\hline J0223.6-0409 & 022336.84 & 1.10 & -040933.15 & 1.76 & $M$ & $9.7 \pm 1.0$ & $5.2 \pm 0.7$ & $<4.5$ & $<3.7$ & $67.9 \pm 21.7$ & -0.71 \\
\hline & 022337.57 & 1.09 & -040935.66 & 1.78 & $C$ & & $4.5 \pm 0.7$ & $<5.1$ & $<3.6$ & $35.7 \pm 11.5$ & \\
\hline J0223.6-0213* & 022337.89 & 1.07 & -021356.48 & 1.75 & $S$ & $83.1 \pm 8.6$ & $83.1 \pm 8.6$ & $10.8 \pm 1.2$ & $<1.7$ & $54.3 \pm 0.8$ & -1.07 \\
\hline J0223.6-0224 & 022337.95 & 1.14 & -022448.47 & 1.94 & $S$ & $12.8 \pm 2.2$ & $12.8 \pm 2.2$ & $9.1 \pm 5.4$ & $<4.6$ & $19.3 \pm 7.0$ & $<-1.00$ \\
\hline J0223.6-0506 & 022338.18 & 1.17 & -050628.51 & 1.75 & $S$ & $26.4 \pm 3.0$ & $26.4 \pm 3.0$ & $14.0 \pm 2.6$ & $<2.9$ & $85.7 \pm 1.9$ & -0.74 \\
\hline J0223.7-0310 & 022340.10 & 1.07 & -031026.22 & 1.78 & $M$ & $8.2 \pm 1.0$ & $4.7 \pm 0.7$ & $<4.7$ & $<2.9$ & $167.8 \pm 8.3$ & $<-0.69$ \\
\hline & 022339.91 & 1.10 & -031025.00 & 1.95 & $C$ & & $3.5 \pm 0.7$ & $<8.5$ & $<4.4$ & $175.4 \pm 9.4$ & \\
\hline J0223.7-0239* & 022341.65 & 1.06 & -023945.77 & 1.75 & $M$ & $205.2 \pm 19.8$ & $197.9 \pm 19.8$ & $4.0 \pm 0.2$ & $1.7 \pm 0.3$ & $35.0 \pm 1.9$ & -0.97 \\
\hline & 022341.23 & 1.07 & -023951.77 & 1.77 & $C$ & & $7.4 \pm 1.0$ & $<4.4$ & $<2.7$ & $9.2 \pm 7.1$ & \\
\hline J0223.7-0411 & 022342.80 & 1.07 & -041159.94 & 1.76 & $S$ & $14.2 \pm 1.6$ & $14.2 \pm 1.6$ & $3.7 \pm 1.7$ & $<2.8$ & $30.8 \pm 10.0$ & -0.73 \\
\hline J0223.7-0331 & 022344.17 & 1.07 & -033132.97 & 1.81 & $S$ & $4.1 \pm 0.7$ & $4.1 \pm 0.7$ & $<5.9$ & $<3.0$ & $0.8 \pm 6.8$ & $<-0.21$ \\
\hline J0223.7-0224 & 022344.55 & 1.11 & -022452.09 & 1.85 & $M$ & $10.1 \pm 1.1$ & $5.4 \pm 0.9$ & $<7.3$ & $<2.7$ & $26.9 \pm 3.4$ & -0.64 \\
\hline & 022344.71 & 1.07 & -022452.39 & 1.81 & $C$ & & $4.7 \pm 0.7$ & $<6.1$ & $<2.4$ & $18.1 \pm 3.7$ & \\
\hline J0223.7-0232 & 022344.65 & 1.19 & -023245.70 & 1.91 & $S$ & $4.9 \pm 1.1$ & $4.9 \pm 1.1$ & $<8.1$ & $<6.1$ & $159.2 \pm 30.3$ & $<-0.34$ \\
\hline $\mathrm{J} 0223.8-0340 *$ & 022345.44 & 1.06 & -034042.18 & 1.75 & $S$ & $106.5 \pm 10.7$ & $106.5 \pm 10.7$ & $5.8 \pm 0.3$ & $2.6 \pm 0.4$ & $103.7 \pm 1.8$ & -0.72 \\
\hline J0223.8-0332* & 022346.58 & 1.06 & -033236.03 & 1.75 & $M$ & $111.5 \pm 10.6$ & $106.0 \pm 10.6$ & $1.6 \pm 0.4$ & $1.1 \pm 0.6$ & $65.8 \pm 28.3$ & -0.52 \\
\hline & 022347.01 & 1.07 & -033239.80 & 1.76 & $C$ & & $5.5 \pm 0.7$ & $<3.9$ & $<2.6$ & $142.3 \pm 8.3$ & \\
\hline J0223.8-0551 & 022348.08 & 1.06 & -055114.65 & 1.75 & $S$ & $129.9 \pm 13.1$ & $129.9 \pm 13.1$ & $2.2 \pm 0.5$ & $<1.4$ & $19.1 \pm 7.2$ & -0.22 \\
\hline J0223.8-0412* & 022349.08 & 1.06 & -041211.53 & 1.75 & $M$ & $85.6 \pm 6.3$ & $43.1 \pm 4.5$ & $6.8 \pm 0.8$ & $4.0 \pm 0.8$ & $151.9 \pm 5.2$ & -0.77 \\
\hline & 022349.83 & 1.06 & -041230.83 & 1.75 & $C$ & & $42.5 \pm 4.4$ & $5.3 \pm 0.7$ & $4.2 \pm 0.7$ & $115.5 \pm 12.4$ & \\
\hline J0223.8-0531 & 022349.80 & 1.09 & -053105.56 & 1.77 & $S$ & $8.1 \pm 1.1$ & $8.1 \pm 1.1$ & $<4.6$ & $<3.5$ & $39.6 \pm 12.1$ & -0.85 \\
\hline J0223.9-0522 & 022352.63 & 1.06 & -052225.48 & 1.75 & $S$ & $26.6 \pm 2.7$ & $26.6 \pm 2.7$ & $<2.3$ & $<2.1$ & $37.9 \pm 9.6$ & -0.65 \\
\hline
\end{tabular}


Table A.1. continued.

\begin{tabular}{|c|c|c|c|c|c|c|c|c|c|c|c|}
\hline Name & $\begin{array}{c}\text { RA } \\
\text { J2000 }\end{array}$ & $\begin{array}{c}\sigma_{\alpha} \\
\operatorname{arcsec}\end{array}$ & $\begin{array}{c}\text { Dec } \\
\text { J2000 }\end{array}$ & $\begin{array}{c}\sigma_{\delta} \\
\operatorname{arcsec}\end{array}$ & Type & $\begin{array}{l}S_{\text {int }} \\
\text { mJy }\end{array}$ & $\begin{array}{c}S_{\text {comp }} \\
\text { mJy }\end{array}$ & $\begin{array}{c}\text { Major axis } \\
\text { arcsec }\end{array}$ & $\begin{array}{c}\text { Minor axis } \\
\text { arcsec }\end{array}$ & $\begin{array}{c}\text { Position } \\
\text { Angle }\end{array}$ & $\begin{array}{c}\text { Spectral } \\
\text { index } \alpha_{325}^{1400}\end{array}$ \\
\hline J0223.9-0445 & 022355.02 & 1.12 & -044545.70 & 2.01 & $S$ & $4.6 \pm 0.9$ & $4.6 \pm 0.9$ & $<9.4$ & $<3.6$ & $17.4 \pm 4.4$ & $<-0.30$ \\
\hline \multirow[t]{2}{*}{ J0223.9-0254 } & 022356.45 & 1.14 & -025431.36 & 1.76 & $M$ & $7.8 \pm 1.0$ & $4.1 \pm 0.7$ & $<5.5$ & $<3.8$ & $100.5 \pm 14.2$ & -0.88 \\
\hline & 022356.45 & 1.23 & -025434.48 & 1.77 & $C$ & & $3.7 \pm 0.7$ & $<7.1$ & $<3.9$ & $71.8 \pm 9.1$ & \\
\hline \multirow[t]{2}{*}{ J0224.0-0441* } & 022357.03 & 1.06 & -044112.64 & 1.75 & $M$ & $219.3 \pm 21.0$ & $209.4 \pm 21.0$ & $3.7 \pm 0.2$ & $1.9 \pm 0.3$ & $19.1 \pm 2.2$ & -0.99 \\
\hline & 022357.49 & 1.27 & -044115.68 & 2.03 & $C$ & & $9.9 \pm 1.8$ & $9.3 \pm 7.8$ & $<3.8$ & $33.4 \pm 3.9$ & \\
\hline J0224.0-0353 & 022359.37 & 1.07 & -035358.38 & 1.75 & $S$ & $14.5 \pm 1.6$ & $14.5 \pm 1.6$ & $<2.9$ & $<2.5$ & $43.0 \pm 11.0$ & -0.96 \\
\hline \multirow[t]{2}{*}{$\mathrm{J} 0224.0-0325$} & 022400.43 & 1.11 & -032536.51 & 1.78 & $M$ & $9.1 \pm 1.1$ & $5.9 \pm 0.8$ & $<5.6$ & $<3.4$ & $44.3 \pm 6.6$ & $<-0.76$ \\
\hline & 022400.66 & 1.54 & -032535.75 & 1.91 & $C$ & & $3.2 \pm 0.7$ & $<10.9$ & $<5.0$ & $122.2 \pm 7.0$ & \\
\hline J0224.0-0238 & 022401.16 & 1.21 & -023849.18 & 1.78 & $S$ & $4.6 \pm 0.8$ & $4.6 \pm 0.8$ & $<6.9$ & $<4.6$ & $71.2 \pm 13.7$ & $<-0.29$ \\
\hline J0224.0-0320 & 022401.20 & 1.22 & -032029.71 & 1.79 & $S$ & $4.9 \pm 0.8$ & $4.9 \pm 0.8$ & $<7.1$ & $<4.8$ & $68.5 \pm 12.4$ & -0.53 \\
\hline J0224.1-0433 & 022403.74 & 1.25 & -043304.96 & 1.82 & $S$ & $9.1 \pm 1.5$ & $9.1 \pm 1.5$ & $8.5 \pm 4.4$ & $<5.3$ & $61.6 \pm 12.8$ & -0.59 \\
\hline J0224.1-0202 & 022405.43 & 1.06 & -020222.82 & 1.75 & $S$ & $30.1 \pm 3.2$ & $30.1 \pm 3.2$ & $<2.3$ & $\ldots$ & $\ldots$ & -1.02 \\
\hline J0224.1-0512 & 022405.51 & 1.07 & -051228.29 & 1.76 & $S$ & $11.0 \pm 1.3$ & $11.0 \pm 1.3$ & $<3.2$ & $<3.0$ & $41.0 \pm 32.3$ & -0.59 \\
\hline J0224.1-0227 & 022405.77 & 1.10 & -022731.99 & 1.80 & $S$ & $5.7 \pm 1.0$ & $5.7 \pm 1.0$ & $<5.6$ & $<4.5$ & $173.0 \pm 27.8$ & -0.48 \\
\hline J0224.1-0319 & 022406.71 & 1.18 & -031906.61 & 1.89 & $S$ & $3.3 \pm 0.7$ & $3.3 \pm 0.7$ & $<7.5$ & $<6.2$ & $10.2 \pm 42.1$ & $<-0.06$ \\
\hline J0224.1-0259 & 022407.84 & 1.11 & -025931.15 & 1.77 & $S$ & $6.3 \pm 0.9$ & $6.3 \pm 0.9$ & $<5.0$ & $<4.1$ & $74.0 \pm 21.9$ & -0.43 \\
\hline $\mathrm{J} 0224.2-0450$ & 022410.21 & 1.09 & -045032.89 & 1.76 & $S$ & $6.0 \pm 0.8$ & $6.0 \pm 0.8$ & $<4.1$ & $<3.5$ & $101.4 \pm 26.6$ & -0.15 \\
\hline \multirow[t]{2}{*}{ J0224.2-0446* } & 022410.29 & 1.06 & -044607.72 & 1.75 & $M$ & $66.5 \pm 5.3$ & $46.9 \pm 4.8$ & $4.8 \pm 0.6$ & $<1.8$ & $99.0 \pm 3.7$ & -0.88 \\
\hline & 022409.51 & 1.08 & -044605.45 & 1.76 & $C$ & & $19.6 \pm 2.2$ & $5.2 \pm 1.6$ & $3.9 \pm 1.7$ & $102.0 \pm 27.7$ & \\
\hline J0224.2-0433 & 022410.42 & 1.37 & -043318.83 & 1.88 & $S$ & $3.3 \pm 0.7$ & $3.3 \pm 0.7$ & $<8.8$ & $<6.8$ & $117.7 \pm 30.7$ & $<-0.07$ \\
\hline J0224.2-0350 & 022410.81 & 1.06 & -035017.09 & 1.75 & $S$ & $18.7 \pm 2.0$ & $18.7 \pm 2.0$ & $<2.4$ & $<2.2$ & $62.9 \pm 14.7$ & -0.76 \\
\hline J0224.2-0307 & 022411.85 & 1.07 & -030744.44 & 1.76 & $S$ & $18.1 \pm 2.0$ & $18.1 \pm 2.0$ & $3.8 \pm 1.6$ & $<2.5$ & $34.4 \pm 5.5$ & -0.55 \\
\hline J0224.2-0355 & 022412.14 & 1.14 & -035557.60 & 1.80 & $S$ & $4.5 \pm 0.7$ & $4.5 \pm 0.7$ & $<5.9$ & $<4.9$ & $41.8 \pm 30.0$ & -0.01 \\
\hline \multirow[t]{3}{*}{ J0224.2-0222 } & 022413.92 & 1.07 & -022219.90 & 1.75 & $M$ & $36.3 \pm 2.7$ & $17.2 \pm 1.9$ & $<3.1$ & $<2.7$ & $61.9 \pm 13.7$ & -0.79 \\
\hline & 022411.07 & 1.07 & -022234.07 & 1.75 & $C$ & & $13.7 \pm 1.6$ & $<3.2$ & $<2.5$ & $61.8 \pm 10.2$ & \\
\hline & 022411.08 & 1.23 & -022236.95 & 1.75 & $C$ & & $5.4 \pm 0.9$ & $<7.0$ & $<2.9$ & $90.1 \pm 4.5$ & \\
\hline \multirow[t]{5}{*}{ J0224.2-0528* } & 022415.02 & 1.06 & -052845.79 & 1.75 & $M$ & $494.5 \pm 24.9$ & $158.0 \pm 16.0$ & $7.9 \pm 0.4$ & $4.5 \pm 0.4$ & $143.3 \pm 2.1$ & -0.72 \\
\hline & 022414.52 & 1.09 & -052833.00 & 1.77 & $C$ & & $131.1 \pm 14.0$ & $16.2 \pm 1.5$ & $9.4 \pm 1.0$ & $134.9 \pm 3.3$ & \\
\hline & 022413.46 & 1.10 & -052808.37 & 1.78 & $C$ & & $87.0 \pm 9.7$ & $15.3 \pm 2.0$ & $7.7 \pm 1.2$ & $142.4 \pm 3.4$ & \\
\hline & 022412.60 & 1.06 & -052755.23 & 1.75 & $C$ & & $66.2 \pm 6.7$ & $3.4 \pm 0.7$ & $<1.9$ & $34.5 \pm 7.3$ & \\
\hline & 022412.85 & 1.07 & -052757.70 & 1.77 & $C$ & & $52.2 \pm 5.7$ & $10.9 \pm 1.6$ & $<2.5$ & $160.3 \pm 2.1$ & \\
\hline J0224.3-0342 & 022415.63 & 1.10 & -034225.92 & 1.81 & $S$ & $3.6 \pm 0.6$ & $3.6 \pm 0.6$ & $<5.8$ & $<4.5$ & $19.4 \pm 21.2$ & $<-0.13$ \\
\hline J0224.3-0431 & 022415.96 & 1.22 & -043142.58 & 1.86 & $S$ & $3.0 \pm 0.6$ & $3.0 \pm 0.6$ & $<7.9$ & $<5.3$ & $43.9 \pm 17.6$ & $<0.01$ \\
\hline $\mathrm{J} 0224.3-0200$ & 022416.66 & 1.23 & -020035.49 & 1.97 & $S$ & $5.7 \pm 1.3$ & $5.7 \pm 1.3$ & $<8.6$ & $<6.9$ & $4.7 \pm 36.6$ & $<-0.44$ \\
\hline J0224.3-0243* & 022418.40 & 1.07 & -024329.05 & 1.76 & $M$ & $142.4 \pm 8.5$ & $41.4 \pm 4.4$ & $8.1 \pm 1.1$ & $<2.2$ & $141.7 \pm 2.3$ & -0.12 \\
\hline & 022417.74 & 1.13 & -024313.41 & 1.81 & $C$ & & $34.4 \pm 4.4$ & $11.7 \pm 2.7$ & $8.1 \pm 2.1$ & $148.0 \pm 12.4$ & \\
\hline & 022418.87 & 1.21 & -024342.55 & 1.79 & $C$ & & $30.9 \pm 4.0$ & $14.1 \pm 3.4$ & $5.7 \pm 2.1$ & $118.1 \pm 5.0$ & \\
\hline & 022417.23 & 1.21 & -024304.72 & 1.82 & $C$ & & $26.5 \pm 3.8$ & $11.9 \pm 3.5$ & $7.5 \pm 2.6$ & $55.1 \pm 12.8$ & \\
\hline & 022418.05 & 1.24 & -024340.97 & 2.09 & $C$ & & $9.2 \pm 1.9$ & $10.6 \pm 6.8$ & $<6.9$ & $168.3 \pm 16.1$ & \\
\hline J0224.3-0300* & 022418.84 & 1.06 & -030022.15 & 1.75 & $M$ & $79.1 \pm 5.9$ & $55.4 \pm 5.6$ & $4.6 \pm 0.5$ & $1.6 \pm 1.0$ & $26.5 \pm 4.2$ & -0.86 \\
\hline & 022419.10 & 1.07 & -030012.85 & 1.75 & $C$ & & $14.4 \pm 1.6$ & $<3.1$ & $<2.2$ & $74.0 \pm 5.1$ & \\
\hline & 022419.14 & 1.10 & -030015.62 & 1.75 & $C$ & & $9.4 \pm 1.1$ & $<4.4$ & $<2.0$ & $97.7 \pm 2.3$ & \\
\hline J0224.3-0502 & 022419.06 & 1.22 & -050236.42 & 1.77 & $S$ & $13.0 \pm 1.8$ & $13.0 \pm 1.8$ & $9.9 \pm 3.6$ & $<3.6$ & $74.5 \pm 4.0$ & -0.82 \\
\hline J0224.4-0311 & 022421.54 & 1.12 & -031125.32 & 1.78 & $S$ & $6.1 \pm 0.9$ & $6.1 \pm 0.9$ & $<5.4$ & $<4.1$ & $54.5 \pm 16.8$ & $<-0.49$ \\
\hline J0224.4-0425* & 022421.59 & 1.06 & -042549.99 & 1.75 & $M$ & $83.7 \pm 5.9$ & $48.6 \pm 4.9$ & $2.9 \pm 0.6$ & $2.1 \pm 0.7$ & $67.5 \pm 15.7$ & -0.96 \\
\hline & 022420.22 & 1.06 & -042542.57 & 1.75 & $C$ & & $31.4 \pm 3.2$ & $3.6 \pm 0.8$ & $1.9 \pm 1.2$ & $52.7 \pm 11.5$ & \\
\hline & 022420.78 & 1.16 & -042544.85 & 1.82 & $C$ & & $3.8 \pm 0.7$ & $<6.5$ & $<5.4$ & $35.5 \pm 38.6$ & \\
\hline J0224.4-0403 & 022425.83 & 1.23 & -040327.12 & 2.06 & $S$ & $2.6 \pm 0.5$ & $2.6 \pm 0.5$ & $<10.6$ & $<3.9$ & $28.7 \pm 4.5$ & $<0.09$ \\
\hline J0224.5-0326 & 022427.33 & 1.65 & -032656.40 & 1.82 & $M$ & $11.6 \pm 1.7$ & $7.6 \pm 1.5$ & $12.1 \pm 7.0$ & $<6.1$ & $94.0 \pm 9.8$ & -0.30 \\
\hline & 022427.69 & 1.13 & -032656.65 & 1.79 & $C$ & & $4.0 \pm 0.7$ & $<5.8$ & $<4.4$ & $50.1 \pm 20.8$ & \\
\hline J0224.5-0243* & 022427.50 & 1.06 & -024305.73 & 1.75 & $M$ & $115.1 \pm 10.9$ & $108.1 \pm 10.8$ & $<1.1$ & $<1.1$ & $81.5 \pm 29.5$ & -0.59 \\
\hline & 022427.11 & 1.07 & -024310.00 & 1.78 & $C$ & & $7.0 \pm 0.9$ & $<4.8$ & $<2.7$ & $156.1 \pm 5.3$ & \\
\hline J0224.5-0449 & 022428.76 & 1.09 & -044952.30 & 1.76 & $M$ & $24.2 \pm 2.4$ & $15.2 \pm 1.8$ & $6.1 \pm 1.9$ & $4.3 \pm 2.0$ & $75.1 \pm 21.5$ & -0.49 \\
\hline & 022427.49 & 1.55 & -044952.95 & 1.81 & $C$ & & $9.0 \pm 1.6$ & $13.8 \pm 6.0$ & $<5.7$ & $91.2 \pm 7.7$ & \\
\hline J0224.5-0307 & 022428.99 & 1.07 & -030720.22 & 1.76 & $S$ & $9.0 \pm 1.1$ & $9.0 \pm 1.1$ & $<3.6$ & $<3.0$ & $43.0 \pm 13.8$ & -0.85 \\
\hline J0224.5-0504 & 022429.20 & 1.07 & -050423.21 & 1.76 & $M$ & $20.9 \pm 1.9$ & $15.2 \pm 1.7$ & $4.6 \pm 1.8$ & $<2.4$ & $129.7 \pm 3.7$ & -1.13 \\
\hline & 022429.31 & 1.10 & -050421.43 & 1.77 & $C$ & & $5.7 \pm 0.8$ & $<5.3$ & $<3.0$ & $43.7 \pm 5.9$ & \\
\hline J0224.5-0335 & 022429.68 & 1.09 & -033530.45 & 1.77 & $S$ & $5.0 \pm 0.7$ & $5.0 \pm 0.7$ & $<4.7$ & $<3.7$ & $50.7 \pm 19.1$ & -0.59 \\
\hline $\mathrm{J} 0224.5-0314$ & 022430.23 & 1.13 & -031454.15 & 1.83 & $S$ & $4.3 \pm 0.7$ & $4.3 \pm 0.7$ & $<6.5$ & $<5.0$ & $25.0 \pm 20.8$ & $<-0.24$ \\
\hline J0224.5-0219* & 022430.33 & 1.09 & -021933.15 & 1.75 & $M$ & $112.7 \pm 9.2$ & $84.2 \pm 8.8$ & $14.6 \pm 1.3$ & $3.1 \pm 0.8$ & $104.7 \pm 1.1$ & -0.50 \\
\hline & 022430.77 & 1.06 & -021934.38 & 1.76 & $C$ & & $23.7 \pm 2.6$ & $3.6 \pm 1.8$ & $<2.2$ & $15.5 \pm 3.2$ & \\
\hline & 022430.68 & 1.31 & -021925.13 & 1.91 & $C$ & & $4.8 \pm 1.0$ & $<9.6$ & $<4.0$ & $134.6 \pm 5.9$ & \\
\hline J0224.5-0534 & 022432.15 & 1.08 & -053430.35 & 1.77 & $S$ & $13.7 \pm 1.7$ & $13.7 \pm 1.7$ & $<4.2$ & $<3.3$ & $34.5 \pm 12.5$ & 0.12 \\
\hline J0224.6-0354 & 022432.92 & 1.11 & -035438.69 & 1.79 & $S$ & $38.3 \pm 11.8^{*}$ & $6.0 \pm 0.9$ & $<5.5$ & $<4.2$ & $38.6 \pm 15.8$ & -0.09 \\
\hline J0224.6-0334 & 022432.95 & 1.09 & -033412.55 & 1.76 & $S$ & $67.2 \pm 10.6^{*}$ & $39.1 \pm 4.3$ & $12.1 \pm 1.6$ & $5.2 \pm 1.0$ & $54.9 \pm 3.0$ & -0.73 \\
\hline J0224.6-0408 & 022433.84 & 1.07 & -040852.93 & 1.76 & $S$ & $10.4 \pm 1.2$ & $10.4 \pm 1.2$ & $<3.5$ & $<3.2$ & $41.4 \pm 24.8$ & -0.90 \\
\hline J0224.6-0310 & 022434.60 & 1.25 & -031059.74 & 1.81 & $S$ & $4.6 \pm 0.8$ & $4.6 \pm 0.8$ & $<7.6$ & $<4.9$ & $119.8 \pm 12.0$ & $<-0.30$ \\
\hline J0224.6-0414 & 022434.96 & 1.09 & -041422.59 & 1.79 & $S$ & $2.8 \pm 0.5$ & $2.8 \pm 0.5$ & $<5.3$ & $<4.1$ & $21.4 \pm 27.7$ & -0.12 \\
\hline J0224.6-0413 & 022436.35 & 1.14 & -041302.18 & 1.82 & $S$ & $3.0 \pm 0.6$ & $3.0 \pm 0.6$ & $<6.1$ & $<5.5$ & $-1.0 \pm 73.2$ & $<-0.01$ \\
\hline J0224.7-0357 & 022439.67 & 1.19 & -035741.38 & 1.77 & $S$ & $3.2 \pm 0.6$ & $3.2 \pm 0.6$ & $<6.4$ & $<4.3$ & $81.6 \pm 14.5$ & $<-0.05$ \\
\hline J0224.7-0347 & 022439.83 & 1.11 & -034710.64 & 1.88 & $M$ & $15.4 \pm 1.5$ & $7.8 \pm 1.2$ & $8.8 \pm 4.4$ & $<4.1$ & $18.2 \pm 6.0$ & -0.52 \\
\hline & 022439.61 & 1.07 & -034712.64 & 1.76 & $C$ & & $7.6 \pm 0.9$ & $<3.5$ & $<3.1$ & $1.7 \pm 21.2$ & \\
\hline J0224.7-0209* & 022441.08 & 1.09 & -020953.06 & 1.76 & $M$ & $47.7 \pm 3.8$ & $23.5 \pm 2.8$ & $4.5 \pm 2.2$ & $<3.3$ & $117.1 \pm 10.3$ & -0.37 \\
\hline & 022441.12 & 1.07 & -020949.32 & 1.75 & $C$ & & $17.9 \pm 2.0$ & $<2.9$ & $<2.7$ & $91.1 \pm 27.9$ & \\
\hline
\end{tabular}


C. Tasse et al.: The low-frequency counterpart of the XMM large scale structure survey, Online Material p 16

Table A.1. continued.

\begin{tabular}{|c|c|c|c|c|c|c|c|c|c|c|c|}
\hline Name & $\begin{array}{c}\text { RA } \\
\text { J2000 }\end{array}$ & $\begin{array}{c}\sigma_{\alpha} \\
\operatorname{arcsec}\end{array}$ & $\begin{array}{c}\text { Dec } \\
\text { J2000 }\end{array}$ & $\begin{array}{c}\sigma_{\delta} \\
\operatorname{arcsec}\end{array}$ & Type & $\begin{array}{l}S_{\text {int }} \\
\text { mJy }\end{array}$ & $\begin{array}{c}S_{\text {comp }} \\
\text { mJy }\end{array}$ & $\begin{array}{c}\text { Major axis } \\
\text { arcsec }\end{array}$ & $\begin{array}{c}\text { Minor axis } \\
\text { arcsec }\end{array}$ & $\begin{array}{c}\text { Position } \\
\text { Angle }\end{array}$ & $\begin{array}{c}\text { Spectral } \\
\text { index } \alpha_{325}^{1400}\end{array}$ \\
\hline & 022441.52 & 1.54 & -021020.01 & 1.89 & $\bar{C}$ & & $6.3 \pm 1.4$ & $<10.2$ & $<7.1$ & $108.9 \pm 20.7$ & \\
\hline J0224.7-0311 & 022442.40 & 1.09 & -031136.99 & 1.78 & $S$ & $5.2 \pm 0.8$ & $5.2 \pm 0.8$ & $<5.0$ & $<4.1$ & $164.1 \pm 27.8$ & $<-0.38$ \\
\hline J0224.7-0330 & 022444.28 & 1.07 & -033016.70 & 1.75 & $S$ & $14.8 \pm 1.6$ & $14.8 \pm 1.6$ & $<2.8$ & $<2.5$ & $53.0 \pm 15.3$ & -0.99 \\
\hline J0224.7-0351 & 022444.74 & 1.14 & -035140.72 & 1.77 & $S$ & $8.3 \pm 1.2$ & $8.3 \pm 1.2$ & $6.0 \pm 3.3$ & $<4.0$ & $65.4 \pm 10.0$ & -0.63 \\
\hline \multirow[t]{8}{*}{ J0224.8-0302* } & 022446.04 & 1.06 & -030156.81 & 1.75 & $M$ & $397.7 \pm 21.9$ & $183.7 \pm 18.4$ & $2.4 \pm 0.2$ & $2.0 \pm 0.2$ & $169.8 \pm 11.9$ & -0.66 \\
\hline & 022445.47 & 1.06 & -030233.74 & 1.75 & $C$ & & $93.2 \pm 9.4$ & $4.1 \pm 0.3$ & $<1.3$ & $34.0 \pm 2.2$ & \\
\hline & 022445.61 & 1.06 & -030225.75 & 1.75 & $C$ & & $57.6 \pm 5.8$ & $3.7 \pm 0.5$ & $<1.6$ & $26.7 \pm 3.5$ & \\
\hline & 022445.47 & 1.30 & -030205.61 & 1.77 & $C$ & & $25.7 \pm 3.3$ & $17.3 \pm 3.9$ & $4.2 \pm 2.0$ & $102.2 \pm 2.8$ & \\
\hline & 022444.90 & 1.20 & -030251.87 & 1.90 & $C$ & & $16.4 \pm 2.5$ & $14.1 \pm 4.5$ & $5.3 \pm 2.9$ & $33.9 \pm 6.4$ & \\
\hline & 022445.58 & 1.09 & -030201.18 & 1.76 & $C$ & & $11.1 \pm 1.3$ & $<4.0$ & $<3.1$ & $96.8 \pm 9.8$ & \\
\hline & 022445.76 & 1.25 & -030219.54 & 1.80 & $C$ & & $5.3 \pm 0.9$ & $<7.8$ & $<3.4$ & $59.8 \pm 4.5$ & \\
\hline & 022444.88 & 1.30 & -030259.60 & 1.93 & $C$ & & $4.7 \pm 0.9$ & $8.7 \pm 6.4$ & $<5.9$ & $139.3 \pm 12.7$ & \\
\hline \multirow[t]{2}{*}{ J0224.8-0408 } & 022447.04 & 1.10 & -040851.88 & 1.77 & $M$ & $6.2 \pm 0.6$ & $3.3 \pm 0.5$ & $<5.0$ & $<3.7$ & $51.8 \pm 19.0$ & $<-0.50$ \\
\hline & 022446.91 & 1.07 & -040849.03 & 1.84 & $C$ & & $3.0 \pm 0.3$ & $<6.7$ & $<0.1$ & $164.1 \pm 0.0$ & \\
\hline \multirow[t]{3}{*}{ J0224.8-0545* } & 022447.98 & 1.07 & -054520.83 & 1.76 & $M$ & $125.5 \pm 9.4$ & $64.1 \pm 6.8$ & $6.5 \pm 1.0$ & $4.7 \pm 1.0$ & $156.2 \pm 10.1$ & -0.71 \\
\hline & 022449.65 & 1.12 & -054547.96 & 1.76 & $C$ & & $55.0 \pm 6.4$ & $11.7 \pm 2.1$ & $5.4 \pm 1.5$ & $108.3 \pm 4.6$ & \\
\hline & 022448.34 & 1.39 & -054529.75 & 1.84 & $C$ & & $6.4 \pm 1.4$ & $<8.8$ & $<6.2$ & $106.7 \pm 21.4$ & \\
\hline J0224.8-0326 & 022448.38 & 1.10 & -032611.39 & 1.77 & $S$ & $6.4 \pm 0.9$ & $6.4 \pm 0.9$ & $<4.7$ & $<4.2$ & $51.6 \pm 39.2$ & $<-0.51$ \\
\hline J0224.8-0221* & 022448.75 & 1.06 & -022119.08 & 1.76 & $S$ & $83.0 \pm 8.6$ & $83.0 \pm 8.6$ & $12.0 \pm 1.0$ & $<1.8$ & $26.2 \pm 0.9$ & -0.49 \\
\hline J0224.8-0353 & 022450.68 & 1.07 & -035358.85 & 1.78 & $S$ & $5.2 \pm 0.7$ & $5.2 \pm 0.7$ & $<4.8$ & $<2.6$ & $9.8 \pm 4.3$ & $<-0.38$ \\
\hline J0224.8-0431 & 022450.83 & 1.08 & -043140.73 & 1.76 & $S$ & $5.0 \pm 0.7$ & $5.0 \pm 0.7$ & $<4.4$ & $<3.2$ & $45.8 \pm 10.4$ & $<-0.35$ \\
\hline J0224.9-0428 & 022451.07 & 1.08 & -042901.43 & 1.78 & $S$ & $4.8 \pm 0.7$ & $4.8 \pm 0.7$ & $<5.0$ & $<3.6$ & $16.4 \pm 12.0$ & $<-0.32$ \\
\hline J0224.9-0452 & 022452.00 & 1.12 & -045252.40 & 2.10 & $S$ & $2.8 \pm 0.6$ & $2.8 \pm 0.6$ & $<10.2$ & $<4.9$ & $6.7 \pm 8.6$ & $<0.04$ \\
\hline J0224.9-0434 & 022453.87 & 1.06 & -043414.30 & 1.75 & $S$ & $25.3 \pm 2.6$ & $25.3 \pm 2.6$ & $2.9 \pm 0.9$ & $<2.1$ & $76.0 \pm 18.5$ & -0.61 \\
\hline J0224.9-0332 & 022454.82 & 1.06 & -033204.40 & 1.75 & $S$ & $23.6 \pm 2.4$ & $23.6 \pm 2.4$ & $<2.1$ & $<2.0$ & $62.8 \pm 13.8$ & -0.43 \\
\hline \multirow[t]{2}{*}{$\mathrm{J} 0224.9-0251$} & 022454.85 & 1.17 & -025110.88 & 1.79 & $M$ & $9.2 \pm 1.1$ & $5.0 \pm 0.8$ & $<6.6$ & $<3.6$ & $56.2 \pm 6.9$ & -0.68 \\
\hline & 022454.72 & 1.12 & -025109.17 & 1.79 & $C$ & & $4.2 \pm 0.7$ & $<5.4$ & $<4.7$ & $135.9 \pm 51.6$ & \\
\hline J0224.9-0329 & 022454.90 & 1.08 & -032905.63 & 1.76 & $S$ & $9.3 \pm 1.1$ & $9.3 \pm 1.1$ & $<3.7$ & $<3.4$ & $38.8 \pm 42.6$ & $<-0.78$ \\
\hline J0224.9-0256 & 022455.20 & 1.19 & -025655.74 & 1.77 & $S$ & $9.9 \pm 1.5$ & $9.9 \pm 1.5$ & $6.5 \pm 4.0$ & $<4.0$ & $75.8 \pm 7.5$ & -0.64 \\
\hline J0224.9-0310 & 022455.66 & 1.06 & -031040.48 & 1.76 & $S$ & $16.8 \pm 1.8$ & $16.8 \pm 1.8$ & $2.4 \pm 2.0$ & $<2.5$ & $1.0 \pm 8.8$ & -0.51 \\
\hline J0224.9-0408 & 022456.47 & 1.08 & -040853.98 & 1.77 & $S$ & $4.9 \pm 0.6$ & $4.9 \pm 0.6$ & $<4.1$ & $<3.6$ & $4.1 \pm 28.1$ & $<-0.33$ \\
\hline \multirow{2}{*}{$\mathrm{J} 0225.0-0516^{*}$} & 022457.72 & 1.06 & -051648.34 & 1.75 & $M$ & $111.7 \pm 8.0$ & $56.9 \pm 5.8$ & $4.0 \pm 0.6$ & $<1.7$ & $22.8 \pm 3.9$ & -0.76 \\
\hline & 022457.21 & 1.06 & -051703.00 & 1.75 & $C$ & & $54.8 \pm 5.6$ & $4.9 \pm 0.6$ & $<1.8$ & $45.4 \pm 3.3$ & \\
\hline J0225.0-0435 & 022459.49 & 1.10 & -043536.54 & 1.76 & $S$ & $5.0 \pm 0.7$ & $5.0 \pm 0.7$ & $<4.6$ & $<3.6$ & $89.5 \pm 15.5$ & -0.51 \\
\hline \multirow[t]{2}{*}{ J0225.0-0321* } & 022500.59 & 1.13 & -032108.18 & 1.79 & $M$ & $35.6 \pm 3.6$ & $25.5 \pm 3.2$ & $10.5 \pm 2.5$ & $7.5 \pm 2.1$ & $131.4 \pm 14.2$ & -0.83 \\
\hline & 022459.90 & 1.13 & -032052.61 & 1.82 & $C$ & & $10.2 \pm 1.5$ & $7.6 \pm 3.3$ & $5.1 \pm 3.2$ & $11.3 \pm 26.2$ & \\
\hline J0225.1-0536* & 022505.13 & 1.06 & -053648.02 & 1.75 & $M$ & $596.3 \pm 56.7$ & $566.1 \pm 56.7$ & $4.4 \pm 0.1$ & $2.5 \pm 0.2$ & $5.6 \pm 1.4$ & -0.62 \\
\hline & 022505.39 & 1.15 & -053641.26 & 1.75 & $C$ & & $17.9 \pm 2.1$ & $<5.7$ & $<1.5$ & $91.2 \pm 1.0$ & \\
\hline & 022505.47 & 1.21 & -053638.66 & 1.75 & $C$ & & $12.4 \pm 1.5$ & $<6.6$ & $<1.2$ & $87.5 \pm 0.6$ & \\
\hline J0225.1-0321 & 022505.40 & 1.10 & -032118.32 & 1.76 & $S$ & $5.4 \pm 0.8$ & $5.4 \pm 0.8$ & $<4.5$ & $<3.6$ & $84.3 \pm 17.4$ & $<-0.40$ \\
\hline J0225.1-0519 & 022505.90 & 1.10 & -051959.76 & 1.77 & $S$ & $9.0 \pm 1.2$ & $9.0 \pm 1.2$ & $<4.6$ & $<4.0$ & $48.3 \pm 23.8$ & -0.50 \\
\hline J0225.1-0300 & 022506.20 & 1.27 & -030023.81 & 1.81 & $S$ & $3.6 \pm 0.7$ & $3.6 \pm 0.7$ & $<7.7$ & $<5.0$ & $65.3 \pm 15.1$ & $<-0.12$ \\
\hline J0225.1-0533 & 022506.25 & 1.29 & -053311.31 & 1.88 & $M$ & $40.3 \pm 4.5$ & $25.0 \pm 3.8$ & $10.1 \pm 5.9$ & $<2.9$ & $47.6 \pm 2.2$ & -1.03 \\
\hline & 022506.42 & 1.10 & -053313.50 & 1.79 & C & & $15.3 \pm 2.3$ & $<5.3$ & $<4.4$ & $17.7 \pm 24.8$ & \\
\hline J0225.1-0516 & 022507.12 & 1.16 & -051631.10 & 1.85 & $S$ & $4.1 \pm 0.9$ & $4.1 \pm 0.9$ & $<6.8$ & $<6.0$ & $-1.0 \pm 62.9$ & $<-0.22$ \\
\hline J0225.1-0316 & 022508.14 & 1.12 & -031638.30 & 1.90 & $S$ & $15.7 \pm 2.2$ & $15.7 \pm 2.2$ & $13.9 \pm 4.0$ & $<4.2$ & $160.6 \pm 4.1$ & -0.07 \\
\hline J0225.1-0406 & 022508.32 & 1.11 & -040653.60 & 1.79 & $S$ & $2.9 \pm 0.5$ & $2.9 \pm 0.5$ & $<5.3$ & $<4.6$ & $147.8 \pm 49.6$ & $<0.02$ \\
\hline J0225.2-0401 & 022509.14 & 1.06 & -040102.24 & 1.75 & $S$ & $24.0 \pm 2.5$ & $24.0 \pm 2.5$ & $<2.0$ & $<1.8$ & $31.3 \pm 12.7$ & -0.86 \\
\hline J0225.2-0509 & 022509.72 & 1.18 & -050949.05 & 1.81 & $S$ & $3.6 \pm 0.7$ & $3.6 \pm 0.7$ & $<6.9$ & $<4.9$ & $50.4 \pm 20.0$ & -0.30 \\
\hline J0225.2-0403 & 022510.21 & 1.09 & -040401.46 & 1.76 & $S$ & $5.7 \pm 0.8$ & $5.7 \pm 0.8$ & $<4.3$ & $<3.7$ & $64.8 \pm 23.4$ & -0.56 \\
\hline J0225.2-0203 & 022510.30 & 1.12 & -020325.25 & 1.79 & $S$ & $7.1 \pm 1.3$ & $7.1 \pm 1.3$ & $<5.3$ & $\ldots$ & $\ldots$ & $<-0.59$ \\
\hline J0225.2-0454 & 022511.13 & 1.31 & -045432.45 & 1.78 & $M$ & $33.6 \pm 3.3$ & $20.2 \pm 2.8$ & $15.1 \pm 4.0$ & $5.3 \pm 2.3$ & $81.8 \pm 4.8$ & -0.47 \\
\hline & $\begin{array}{lll}02 & 25 & 10.17\end{array}$ & 1.35 & -045436.10 & 1.83 & $C$ & & $6.7 \pm 1.3$ & $8.3 \pm 5.8$ & $<5.6$ & $114.8 \pm 12.4$ & \\
\hline & 022511.93 & 1.35 & -045425.83 & 1.85 & $C$ & & $6.6 \pm 1.3$ & $8.3 \pm 5.4$ & $<6.7$ & $76.4 \pm 28.1$ & \\
\hline J0225.2-0249 & 022511.34 & 1.23 & -024903.85 & 1.90 & $S$ & $4.0 \pm 0.8$ & $4.0 \pm 0.8$ & $<7.7$ & $<7.0$ & $-1.0 \pm 78.2$ & 0.03 \\
\hline J0225.2-0524 & 022512.08 & 1.18 & -052412.12 & 1.90 & $S$ & $5.7 \pm 1.1$ & $5.7 \pm 1.1$ & $<8.1$ & $<5.1$ & $151.3 \pm 12.7$ & 0.42 \\
\hline J0225.2-0343 & 022512.89 & 1.07 & -034309.77 & 1.76 & $S$ & $7.2 \pm 0.9$ & $7.2 \pm 0.9$ & $<3.7$ & $<3.2$ & $14.9 \pm 18.3$ & $<-0.60$ \\
\hline J0225.2-0229 & 022513.92 & 1.10 & -022916.06 & 1.78 & $M$ & $10.7 \pm 1.3$ & $6.7 \pm 1.0$ & $<5.4$ & $<3.5$ & $137.3 \pm 9.4$ & 0.50 \\
\hline & 022513.82 & 1.40 & -022916.22 & 1.88 & $C$ & & $4.0 \pm 0.8$ & $<9.9$ & $<4.3$ & $53.6 \pm 6.6$ & \\
\hline J0225.2-0301 & 022514.53 & 1.09 & -030106.72 & 1.76 & $S$ & $12.4 \pm 1.5$ & $12.4 \pm 1.5$ & $4.2 \pm 2.3$ & $<3.1$ & $100.2 \pm 8.6$ & -0.30 \\
\hline J0225.2-0436 & 022514.88 & 1.10 & -043615.60 & 1.76 & $M$ & $9.6 \pm 1.0$ & $6.5 \pm 0.8$ & $<4.4$ & $<3.1$ & $81.9 \pm 8.0$ & -0.64 \\
\hline & 022514.96 & 1.45 & -043612.48 & 1.78 & $C$ & & $3.0 \pm 0.5$ & $<9.4$ & $<2.9$ & $72.4 \pm 2.9$ & \\
\hline J0225.3-0233 & 022514.98 & 1.06 & -023347.24 & 1.80 & $S$ & $6.3 \pm 0.7$ & $6.3 \pm 0.7$ & $<5.5$ & $<0.9$ & $3.0 \pm 0.3$ & $<-0.51$ \\
\hline J0225.3-0424 & 022516.52 & 1.07 & -042426.54 & 1.77 & $S$ & $3.9 \pm 0.5$ & $3.9 \pm 0.5$ & $<4.0$ & $<3.3$ & $16.4 \pm 22.9$ & $<-0.18$ \\
\hline J0225.3-0546 & 022516.67 & 1.12 & -054602.61 & 1.94 & $S$ & $6.1 \pm 1.3$ & $6.1 \pm 1.3$ & $<8.3$ & $<4.8$ & $167.6 \pm 12.3$ & $<-0.48$ \\
\hline J0225.3-0417 & 022517.96 & 1.33 & -041754.64 & 1.81 & $S$ & $2.5 \pm 0.5$ & $2.5 \pm 0.5$ & $<8.4$ & $<4.9$ & $65.6 \pm 12.0$ & $<0.13$ \\
\hline J0225.3-0240 & 022518.29 & 1.08 & -024047.82 & 1.75 & $M$ & $21.3 \pm 1.7$ & $9.5 \pm 1.1$ & $<3.5$ & $<2.3$ & $85.4 \pm 5.5$ & -0.56 \\
\hline & 022518.29 & 1.10 & -024050.82 & 1.75 & $C$ & & $8.1 \pm 1.0$ & $<4.7$ & $<2.5$ & $77.5 \pm 4.0$ & \\
\hline & 022518.99 & 1.18 & -024049.11 & 1.92 & $C$ & & $3.8 \pm 0.8$ & $<8.5$ & $<5.0$ & $151.8 \pm 13.1$ & \\
\hline J0225.3-0354 & 022519.85 & 1.06 & -035440.17 & 1.75 & $S$ & $22.3 \pm 2.3$ & $22.3 \pm 2.3$ & $<2.0$ & $<1.8$ & $49.9 \pm 11.2$ & -1.06 \\
\hline J0225.4-0327 & 022521.76 & 1.14 & -032754.00 & 1.83 & $S$ & $2.7 \pm 0.6$ & $2.7 \pm 0.6$ & $<6.5$ & $<5.3$ & $157.7 \pm 39.6$ & $<0.08$ \\
\hline J0225.4-0349 & 022525.19 & 1.29 & -034926.68 & 1.84 & $S$ & $2.7 \pm 0.6$ & $2.7 \pm 0.6$ & $<7.6$ & $<6.6$ & $100.7 \pm 55.2$ & $<0.07$ \\
\hline J0225.4-0248* & 022525.71 & 1.06 & -024814.60 & 1.75 & $M$ & $60.0 \pm 4.3$ & $35.0 \pm 3.6$ & $<1.9$ & $<1.8$ & $57.7 \pm 13.9$ & -0.82 \\
\hline & 022526.57 & 1.07 & -024816.52 & 1.76 & C & & $12.8 \pm 1.5$ & $<3.3$ & $<2.7$ & $177.5 \pm 13.2$ & \\
\hline
\end{tabular}


Table A.1. continued.

\begin{tabular}{|c|c|c|c|c|c|c|c|c|c|c|c|}
\hline Name & $\begin{array}{c}\text { RA } \\
\text { J2000 }\end{array}$ & $\begin{array}{c}\sigma_{\alpha} \\
\operatorname{arcsec}\end{array}$ & $\begin{array}{c}\text { Dec } \\
\text { J2000 }\end{array}$ & $\begin{array}{c}\sigma_{\delta} \\
\operatorname{arcsec}\end{array}$ & Type & $\begin{array}{l}S_{\text {int }} \\
\text { mJy }\end{array}$ & $\begin{array}{c}S_{\text {comp }} \\
\text { mJy }\end{array}$ & $\begin{array}{c}\text { Major axis } \\
\text { arcsec }\end{array}$ & $\begin{array}{c}\text { Minor axis } \\
\text { arcsec }\end{array}$ & $\begin{array}{c}\text { Position } \\
\text { Angle }\end{array}$ & $\begin{array}{c}\text { Spectral } \\
\text { index } \alpha_{325}^{1400}\end{array}$ \\
\hline & 022526.33 & 1.09 & -024816.74 & 1.89 & $C$ & & $12.2 \pm 1.8$ & $9.1 \pm 4.3$ & $<3.7$ & $12.3 \pm 4.3$ & \\
\hline J0225.5-0524 & 022526.97 & 1.18 & -052456.60 & 1.82 & $S$ & $3.8 \pm 0.8$ & $3.8 \pm 0.8$ & $<7.1$ & $<4.7$ & $45.7 \pm 17.3$ & $<-0.16$ \\
\hline J0225.5-0538 & 022527.69 & 1.25 & -053856.21 & 2.07 & $S$ & $4.8 \pm 1.0$ & $4.8 \pm 1.0$ & $<10.5$ & $<5.2$ & $151.9 \pm 8.1$ & $<-0.32$ \\
\hline $\mathrm{J} 0225.5-0513$ & 022527.95 & 1.12 & -051343.94 & 1.79 & $S$ & $5.4 \pm 0.8$ & $5.4 \pm 0.8$ & $<5.6$ & $<4.5$ & $36.8 \pm 21.6$ & $<-0.40$ \\
\hline J0225.5-0536 & 022528.52 & 1.11 & -053656.64 & 1.77 & $S$ & $6.4 \pm 1.0$ & $6.4 \pm 1.0$ & $<5.2$ & $<3.2$ & $55.5 \pm 8.9$ & -0.58 \\
\hline J0225.5-0439 & 022530.22 & 1.18 & -043935.37 & 1.89 & $S$ & $3.1 \pm 0.6$ & $3.1 \pm 0.6$ & $<7.5$ & $<6.2$ & $3.8 \pm 38.9$ & $<-0.03$ \\
\hline J0225.6-0210* & 022534.16 & 1.06 & -021018.59 & 1.75 & $S$ & $790.6 \pm 79.1$ & $790.6 \pm 79.1$ & $1.0 \pm 0.3$ & $<0.7$ & $20.8 \pm 10.4$ & -0.39 \\
\hline \multirow[t]{4}{*}{ J0225.6-0306 } & 022534.98 & 1.09 & -030627.17 & 1.80 & $M$ & $26.6 \pm 2.1$ & $13.0 \pm 1.7$ & $8.6 \pm 2.8$ & $<3.6$ & $154.4 \pm 5.1$ & -0.84 \\
\hline & 022534.41 & 1.08 & -030616.92 & 1.78 & $C$ & & $7.0 \pm 0.9$ & $<4.6$ & $<3.8$ & $173.1 \pm 18.4$ & \\
\hline & 022535.45 & 1.15 & -030635.82 & 1.82 & $C$ & & $3.4 \pm 0.7$ & $<6.2$ & $<5.4$ & $35.4 \pm 55.2$ & \\
\hline & 022535.37 & 1.37 & -030633.05 & 1.95 & $C$ & & $3.1 \pm 0.6$ & $<10.4$ & $<3.5$ & $45.4 \pm 3.9$ & \\
\hline \multirow[t]{2}{*}{ J0225.6-0502 } & 022535.03 & 1.15 & -050209.51 & 1.88 & $M$ & $21.0 \pm 2.7$ & $17.4 \pm 2.6$ & $12.3 \pm 3.9$ & $7.0 \pm 2.8$ & $16.5 \pm 11.2$ & -0.57 \\
\hline & 022534.68 & 1.15 & -050216.70 & 1.84 & $C$ & & $3.6 \pm 0.6$ & $<7.2$ & $<4.0$ & $144.3 \pm 9.1$ & \\
\hline J0225.6-0421 & 022536.28 & 1.14 & -042143.10 & 1.86 & $S$ & $2.4 \pm 0.5$ & $2.4 \pm 0.5$ & $<7.0$ & $<5.2$ & $163.0 \pm 25.3$ & $<0.14$ \\
\hline J0225.6-0512 & 022536.46 & 1.11 & -051259.31 & 1.76 & $S$ & $7.8 \pm 1.0$ & $7.8 \pm 1.0$ & $<4.9$ & $<2.9$ & $83.0 \pm 5.2$ & $<-0.65$ \\
\hline J0225.6-0500 & 022536.48 & 1.07 & -050011.27 & 1.76 & $S$ & $24.3 \pm 2.7$ & $24.3 \pm 2.7$ & $5.7 \pm 1.3$ & $3.6 \pm 1.4$ & $48.7 \pm 12.2$ & -0.51 \\
\hline \multirow[t]{3}{*}{$\mathrm{J} 0225.6-0406$} & 022537.58 & 1.06 & -040650.25 & 1.75 & $M$ & $30.5 \pm 2.2$ & $15.7 \pm 1.7$ & $<2.6$ & $<2.1$ & $55.5 \pm 5.1$ & -0.82 \\
\hline & 022539.17 & 1.08 & -040629.49 & 1.76 & $C$ & & $7.6 \pm 0.9$ & $<4.1$ & $<3.3$ & $47.8 \pm 13.7$ & \\
\hline & 022537.77 & 1.32 & -040646.33 & 1.77 & $C$ & & $7.1 \pm 1.0$ & $8.2 \pm 5.2$ & $<2.8$ & $73.1 \pm 2.3$ & \\
\hline J0225.7-0604 & 022539.33 & 1.23 & -060437.84 & 2.00 & $S$ & $8.8 \pm 1.8$ & $8.8 \pm 1.8$ & $13.0 \pm 6.0$ & $<4.5$ & $149.4 \pm 4.1$ & $<-0.74$ \\
\hline J0225.7-0417 & 022540.00 & 1.10 & -041756.17 & 1.78 & $S$ & $6.0 \pm 0.8$ & $6.0 \pm 0.8$ & $<5.0$ & $<3.9$ & $36.3 \pm 13.6$ & -0.39 \\
\hline J0225.7-0553 & 022540.23 & 1.13 & -055331.78 & 1.87 & $S$ & $5.4 \pm 1.0$ & $5.4 \pm 1.0$ & $<7.6$ & $<3.4$ & $151.2 \pm 5.9$ & $<-0.40$ \\
\hline J0225.7-0216 & 022542.60 & 1.12 & -021606.73 & 1.77 & $S$ & $8.4 \pm 1.4$ & $8.4 \pm 1.4$ & $<5.2$ & $<4.0$ & $103.0 \pm 23.0$ & $<-0.70$ \\
\hline J0225.7-0402 & 022542.70 & 1.12 & -040238.37 & 1.80 & $S$ & $3.2 \pm 0.6$ & $3.2 \pm 0.6$ & $<5.4$ & $<5.0$ & $-1.0 \pm 78.3$ & $<-0.04$ \\
\hline J0225.7-0230 & 022543.24 & 1.10 & -023005.92 & 1.82 & $S$ & $4.6 \pm 0.9$ & $4.6 \pm 0.9$ & $<6.2$ & $<4.5$ & $179.3 \pm 19.7$ & -0.34 \\
\hline J0225.7-0319 & 022543.86 & 1.07 & -031935.00 & 1.76 & $S$ & $9.5 \pm 1.1$ & $9.5 \pm 1.1$ & $<3.2$ & $<2.7$ & $25.0 \pm 15.5$ & -0.77 \\
\hline \multirow[t]{2}{*}{ J0225.7-0311 } & 022543.93 & 1.28 & -031103.86 & 1.82 & $M$ & $9.3 \pm 1.1$ & $4.7 \pm 0.8$ & $7.2 \pm 5.6$ & $<4.8$ & $60.8 \pm 9.4$ & -0.50 \\
\hline & 022545.14 & 1.17 & -031101.62 & 1.79 & $C$ & & $4.6 \pm 0.8$ & $<6.5$ & $<4.4$ & $56.3 \pm 13.6$ & \\
\hline \multirow[t]{2}{*}{ J0225.7-0604 } & 022544.18 & 1.08 & -060425.64 & 1.85 & $M$ & $7.0 \pm 0.8$ & $4.1 \pm 0.6$ & $<6.7$ & $<3.6$ & $2.9 \pm 6.2$ & $<-0.58$ \\
\hline & 022545.65 & 1.15 & -060431.18 & 1.88 & $C$ & & $2.9 \pm 0.5$ & $<8.2$ & $<2.8$ & $147.3 \pm 3.3$ & \\
\hline J0225.7-0316 & 022544.19 & 1.07 & -031632.46 & 1.76 & $S$ & $11.7 \pm 1.3$ & $11.7 \pm 1.3$ & $<3.5$ & $<2.8$ & $19.2 \pm 8.9$ & -0.46 \\
\hline J0225.8-0505 & 022545.38 & 1.11 & -050547.68 & 1.80 & $S$ & $4.0 \pm 0.7$ & $4.0 \pm 0.7$ & $<5.6$ & $<4.8$ & $170.4 \pm 40.0$ & $<-0.20$ \\
\hline J0225.8-0415 & 022545.76 & 1.12 & -041527.80 & 1.77 & $S$ & $5.5 \pm 0.8$ & $5.5 \pm 0.8$ & $4.0 \pm 3.6$ & $<4.2$ & $75.8 \pm 19.1$ & -0.56 \\
\hline \multirow[t]{2}{*}{ J0225.8-0226 } & 022547.59 & 1.09 & -022628.69 & 1.77 & $M$ & $14.1 \pm 1.5$ & $8.8 \pm 1.2$ & $<4.3$ & $<3.8$ & $49.5 \pm 29.3$ & -0.82 \\
\hline & 022548.36 & 1.17 & -022554.39 & 1.79 & $C$ & & $5.3 \pm 0.9$ & $<6.3$ & $<4.5$ & $63.5 \pm 17.7$ & \\
\hline J0225.8-0258 & 022547.76 & 1.24 & -025818.49 & 1.91 & $M$ & $78.2 \pm 16.1^{*}$ & $14.4 \pm 2.5$ & $9.9 \pm 4.5$ & $7.8 \pm 4.0$ & $163.9 \pm 40.9$ & 0.10 \\
\hline & 022545.82 & 1.10 & -025804.49 & 1.77 & $C$ & & $6.4 \pm 0.9$ & $<4.8$ & $<3.5$ & $130.9 \pm 12.4$ & \\
\hline & 022548.67 & 1.18 & -025820.54 & 1.81 & $C$ & & $5.5 \pm 0.9$ & $5.5 \pm 4.4$ & $<5.6$ & $55.4 \pm 34.5$ & \\
\hline J0225.8-0341 & 022548.41 & 1.12 & -034129.08 & 1.77 & $S$ & $3.2 \pm 0.5$ & $3.2 \pm 0.5$ & $<5.2$ & $<4.1$ & $91.4 \pm 26.4$ & $<-0.05$ \\
\hline J0225.8-0600 & 022549.63 & 1.08 & -060051.69 & 1.76 & $S$ & $17.2 \pm 2.1$ & $17.2 \pm 2.1$ & $<3.8$ & $<3.6$ & $16.9 \pm 46.0$ & -0.14 \\
\hline J0225.8-0400 & 022549.77 & 1.10 & -040023.98 & 1.77 & $S$ & $4.4 \pm 0.7$ & $4.4 \pm 0.7$ & $<4.7$ & $<4.1$ & $128.2 \pm 38.4$ & $<-0.26$ \\
\hline J0225.8-0251 & 022550.08 & 1.07 & -025146.67 & 1.76 & $S$ & $8.2 \pm 1.0$ & $8.2 \pm 1.0$ & $<3.7$ & $<3.0$ & $46.4 \pm 15.1$ & -0.78 \\
\hline J0225.8-0422 & 022550.19 & 1.07 & -042219.94 & 1.75 & $S$ & $11.8 \pm 1.3$ & $11.8 \pm 1.3$ & $<2.9$ & $<2.4$ & $40.2 \pm 8.4$ & -0.69 \\
\hline J0225.9-0336 & 022550.96 & 1.10 & -033632.72 & 1.76 & $S$ & $5.1 \pm 0.7$ & $5.1 \pm 0.7$ & $<4.7$ & $<3.4$ & $58.2 \pm 10.6$ & $<-0.36$ \\
\hline J0225.9-0500 & 022552.94 & 1.13 & -050016.72 & 1.77 & $M$ & $14.0 \pm 1.5$ & $9.4 \pm 1.3$ & $6.3 \pm 3.1$ & $<3.6$ & $119.2 \pm 6.6$ & -0.53 \\
\hline & 022553.52 & 1.11 & -050020.79 & 1.80 & $C$ & & $4.6 \pm 0.8$ & $<5.8$ & $<4.7$ & $24.4 \pm 23.5$ & \\
\hline J0225.9-0157 & 022553.19 & 1.07 & -015759.92 & 1.77 & $S$ & $10.9 \pm 1.5$ & $10.9 \pm 1.5$ & $<4.5$ & $<3.3$ & $18.4 \pm 11.1$ & -0.98 \\
\hline J0225.9-0553 & 022553.76 & 1.17 & -055304.05 & 1.92 & $S$ & $14.1 \pm 2.4$ & $14.1 \pm 2.4$ & $9.7 \pm 4.7$ & $<5.9$ & $11.5 \pm 16.1$ & -0.09 \\
\hline J0225.9-0428 & 022554.78 & 1.08 & -042854.64 & 1.76 & $M$ & $23.5 \pm 2.0$ & $12.7 \pm 1.4$ & $5.1 \pm 1.7$ & $<3.1$ & $42.5 \pm 9.1$ & -0.73 \\
\hline & 022556.08 & 1.18 & -042848.40 & 1.78 & $C$ & & $8.1 \pm 1.2$ & $7.5 \pm 3.4$ & $<4.6$ & $78.0 \pm 11.7$ & \\
\hline & 022555.31 & 1.14 & -042848.48 & 1.85 & $C$ & & $2.7 \pm 0.6$ & $<6.8$ & $<5.5$ & $9.4 \pm 38.1$ & \\
\hline J0225.9-0545* & 022555.35 & 1.06 & -054540.25 & 1.75 & $S$ & $117.0 \pm 11.8$ & $117.0 \pm 11.8$ & $7.6 \pm 0.4$ & $1.4 \pm 0.8$ & $39.3 \pm 1.1$ & -0.46 \\
\hline J0225.9-0247 & 022555.90 & 1.11 & -024727.29 & 1.99 & $S$ & $3.9 \pm 0.7$ & $3.9 \pm 0.7$ & $<8.9$ & $<4.9$ & $178.9 \pm 8.8$ & $<-0.18$ \\
\hline J0225.9-0447 & 022556.04 & 1.10 & -044722.91 & 1.76 & $S$ & $3.6 \pm 0.6$ & $3.6 \pm 0.6$ & $<4.6$ & $<3.8$ & $73.4 \pm 28.7$ & -0.29 \\
\hline J0225.9-0534 & 022556.42 & 1.08 & -053449.82 & 1.78 & $S$ & $6.0 \pm 0.9$ & $6.0 \pm 0.9$ & $<5.0$ & $<3.6$ & $3.6 \pm 13.2$ & 0.34 \\
\hline J0226.0-0500 & 022558.79 & 1.07 & -050052.94 & 1.75 & $S$ & $10.9 \pm 1.2$ & $10.9 \pm 1.2$ & $<3.0$ & $<2.6$ & $16.5 \pm 15.4$ & -0.86 \\
\hline J0226.0-0542 & 022559.43 & 1.13 & -054252.11 & 1.86 & $S$ & $4.8 \pm 1.0$ & $4.8 \pm 1.0$ & $<7.0$ & $<5.2$ & $177.1 \pm 24.9$ & $<-0.31$ \\
\hline J0226.0-0444 & 022559.56 & 1.37 & -044405.44 & 1.77 & $S$ & $15.4 \pm 2.1$ & $15.4 \pm 2.1$ & $16.4 \pm 4.5$ & $<3.9$ & $99.6 \pm 2.8$ & -0.17 \\
\hline J0226.0-0222 & 022600.12 & 1.10 & -022230.03 & 1.78 & $S$ & $6.1 \pm 0.9$ & $6.1 \pm 0.9$ & $<5.5$ & $<3.6$ & $142.6 \pm 11.1$ & $<-0.49$ \\
\hline J0226.0-0512 & 022601.30 & 1.32 & -051207.14 & 1.83 & $S$ & $3.5 \pm 0.8$ & $3.5 \pm 0.8$ & $<8.4$ & $<5.5$ & $63.1 \pm 16.6$ & -0.25 \\
\hline J0226.1-0429 & 022603.07 & 1.08 & -042930.33 & 1.76 & $S$ & $5.9 \pm 0.8$ & $5.9 \pm 0.8$ & $<4.1$ & $<3.1$ & $43.7 \pm 13.0$ & $<-0.46$ \\
\hline J0226.1-0430 & 022603.59 & 1.09 & -043101.14 & 1.79 & $M$ & $11.9 \pm 1.4$ & $8.9 \pm 1.2$ & $5.8 \pm 3.1$ & $<3.8$ & $160.5 \pm 8.4$ & 0.16 \\
\hline & 022603.77 & 1.24 & -043115.65 & 1.95 & $C$ & & $3.0 \pm 0.7$ & $<8.5$ & $<6.9$ & $12.0 \pm 41.2$ & \\
\hline J0226.1-0258 & 022604.67 & 1.17 & -025811.55 & 1.79 & $M$ & $5.9 \pm 0.8$ & $3.0 \pm 0.6$ & $<6.3$ & $<4.4$ & $60.8 \pm 18.3$ & -0.67 \\
\hline & 022604.44 & 1.12 & -025812.50 & 2.09 & $C$ & & $2.8 \pm 0.6$ & $<10.1$ & $<5.1$ & $176.0 \pm 9.3$ & \\
\hline J0226.1-0251 & 022605.13 & 1.08 & -025151.68 & 1.76 & $S$ & $8.5 \pm 1.0$ & $8.5 \pm 1.0$ & $<3.9$ & $<3.1$ & $58.8 \pm 11.8$ & $<-0.71$ \\
\hline J0226.1-0301 & 022605.88 & 1.30 & -030106.71 & 1.82 & $S$ & $3.4 \pm 0.7$ & $3.4 \pm 0.7$ & $<7.8$ & $<5.9$ & $102.3 \pm 26.8$ & $<-0.09$ \\
\hline J0226.1-0233 & 022606.04 & 1.07 & -023348.55 & 1.76 & $S$ & $16.0 \pm 1.8$ & $16.0 \pm 1.8$ & $<3.1$ & $<2.8$ & $2.4 \pm 18.0$ & -0.55 \\
\hline J0226.1-0416 & 022606.50 & 1.28 & -041632.65 & 1.82 & $S$ & $2.8 \pm 0.6$ & $2.8 \pm 0.6$ & $<7.7$ & $<5.7$ & $70.4 \pm 25.7$ & $<0.04$ \\
\hline J0226.1-0532 & 022606.91 & 1.06 & -053216.75 & 1.75 & $S$ & $46.9 \pm 4.8$ & $46.9 \pm 4.8$ & $2.4 \pm 0.8$ & $<1.7$ & $30.0 \pm 6.0$ & -0.07 \\
\hline J0226.1-0235 & 022608.12 & 1.06 & -023515.97 & 1.75 & $S$ & $21.6 \pm 2.3$ & $21.6 \pm 2.3$ & $<2.3$ & $<2.1$ & $55.1 \pm 11.3$ & -0.48 \\
\hline J0226.2-0433 & 022609.09 & 1.06 & -043335.92 & 1.75 & $S$ & $26.4 \pm 2.7$ & $26.4 \pm 2.7$ & $<2.0$ & $<1.9$ & $167.2 \pm 9.4$ & -0.90 \\
\hline J0226.2-0212 & 022609.90 & 1.15 & -021223.68 & 1.91 & $S$ & $4.8 \pm 1.0$ & $4.8 \pm 1.0$ & $<7.8$ & $<5.7$ & $179.3 \pm 24.0$ & $<-0.33$ \\
\hline J0226.2-0204 & 022611.25 & 1.09 & -020407.44 & 1.80 & $S$ & $7.2 \pm 1.2$ & $7.2 \pm 1.2$ & $<5.7$ & $<4.0$ & $5.6 \pm 14.1$ & $<-0.60$ \\
\hline
\end{tabular}


C. Tasse et al.: The low-frequency counterpart of the XMM large scale structure survey, Online Material p 18

Table A.1. continued.

\begin{tabular}{|c|c|c|c|c|c|c|c|c|c|c|c|}
\hline Name & $\begin{array}{c}\text { RA } \\
\text { J2000 }\end{array}$ & $\begin{array}{c}\sigma_{\alpha} \\
\operatorname{arcsec}\end{array}$ & $\begin{array}{c}\text { Dec } \\
\text { J2000 }\end{array}$ & $\begin{array}{c}\sigma_{\delta} \\
\operatorname{arcsec}\end{array}$ & Type & $\begin{array}{l}S_{\text {int }} \\
\text { mJy }\end{array}$ & $\begin{array}{c}S_{\text {comp }} \\
\text { mJy }\end{array}$ & $\begin{array}{c}\text { Major axis } \\
\text { arcsec }\end{array}$ & $\begin{array}{c}\text { Minor axis } \\
\text { arcsec }\end{array}$ & $\begin{array}{c}\text { Position } \\
\text { Angle }\end{array}$ & $\begin{array}{c}\text { Spectral } \\
\text { index } \alpha_{325}^{1400}\end{array}$ \\
\hline \multirow[t]{2}{*}{ J0226.2-0505 } & 022611.35 & 1.16 & -050503.85 & 1.77 & $M$ & $10.4 \pm 1.1$ & $5.2 \pm 0.8$ & $<6.1$ & $<3.3$ & $65.4 \pm 6.2$ & $<-0.85$ \\
\hline & 022611.31 & 1.08 & -050506.99 & 1.76 & $C$ & & $5.2 \pm 0.7$ & $<4.0$ & $<3.6$ & $118.0 \pm 42.9$ & \\
\hline J0226.2-0403 & 022612.67 & 1.07 & -040319.84 & 1.76 & $S$ & $8.4 \pm 1.0$ & $8.4 \pm 1.0$ & $<3.4$ & $<3.0$ & $53.3 \pm 23.1$ & -0.74 \\
\hline J0226.2-0307 & 022614.39 & 1.07 & -030755.42 & 1.75 & $S$ & $13.3 \pm 1.4$ & $13.3 \pm 1.4$ & $<2.7$ & $<2.3$ & $59.5 \pm 10.5$ & -0.44 \\
\hline \multirow[t]{2}{*}{ J0226.3-0422 } & 022615.20 & 1.06 & -042234.64 & 1.75 & $M$ & $34.5 \pm 2.8$ & $21.1 \pm 2.2$ & $<2.3$ & $<2.2$ & $112.1 \pm 20.4$ & -0.84 \\
\hline & 022615.53 & 1.13 & -042230.73 & 1.79 & $C$ & & $13.4 \pm 1.7$ & $9.3 \pm 3.1$ & $<3.0$ & $48.3 \pm 2.9$ & \\
\hline J0226.3-0219 & 022616.88 & 1.22 & -021949.54 & 1.87 & $S$ & $5.0 \pm 1.0$ & $5.0 \pm 1.0$ & $<7.6$ & $<6.0$ & $39.3 \pm 28.0$ & -0.15 \\
\hline J0226.3-0504 & 022617.39 & 1.06 & -050441.90 & 1.75 & $S$ & $17.1 \pm 1.8$ & $17.1 \pm 1.8$ & $<2.6$ & $<2.4$ & $17.5 \pm 16.7$ & -0.52 \\
\hline \multirow[t]{4}{*}{$\mathrm{J} 0226.3-0400 *$} & 022619.09 & 1.12 & -040015.04 & 1.78 & $M$ & $132.7 \pm 10.9$ & $91.5 \pm 9.9$ & $22.1 \pm 2.2$ & $9.5 \pm 1.1$ & $127.8 \pm 2.0$ & -0.28 \\
\hline & 022618.48 & 1.39 & -035926.41 & 2.09 & $C$ & & $19.3 \pm 3.7$ & $15.4 \pm 6.5$ & $9.1 \pm 4.5$ & $30.2 \pm 15.7$ & \\
\hline & 022618.58 & 1.18 & -040007.09 & 1.84 & $C$ & & $14.4 \pm 2.0$ & $11.3 \pm 4.2$ & $<3.2$ & $138.1 \pm 2.7$ & \\
\hline & 022617.23 & 1.23 & -035933.04 & 2.03 & $C$ & & $7.6 \pm 1.6$ & $9.2 \pm 6.5$ & $<6.9$ & $173.5 \pm 20.6$ & \\
\hline J0226.3-0203 & 022619.53 & 1.06 & -020350.36 & 1.75 & $S$ & $23.2 \pm 2.5$ & $23.2 \pm 2.5$ & $<2.5$ & $<2.2$ & $60.5 \pm 14.4$ & -0.65 \\
\hline \multirow[t]{2}{*}{$\mathrm{J} 0226.3-0425^{*}$} & 022619.75 & 1.06 & -042531.89 & 1.75 & $M$ & $115.1 \pm 8.2$ & $60.1 \pm 6.1$ & $4.2 \pm 0.5$ & $3.2 \pm 0.6$ & $54.5 \pm 11.0$ & -0.75 \\
\hline & 022619.88 & 1.06 & -042539.46 & 1.75 & $C$ & & $55.0 \pm 5.6$ & $3.4 \pm 0.5$ & $1.4 \pm 1.0$ & $163.8 \pm 6.8$ & \\
\hline J0226.4-0554 & 022621.03 & 1.14 & -055501.50 & 1.77 & $S$ & $5.1 \pm 0.9$ & $5.1 \pm 0.9$ & $<5.5$ & $<3.9$ & $99.5 \pm 18.9$ & $<-0.36$ \\
\hline J0226.4-0557 & 022625.89 & 1.14 & -055710.90 & 1.82 & $S$ & $5.0 \pm 1.0$ & $5.0 \pm 1.0$ & $<6.3$ & $<5.0$ & $30.9 \pm 34.1$ & $<-0.35$ \\
\hline J0226.5-0332 & 022627.08 & 1.10 & -033300.82 & 1.76 & $S$ & $7.4 \pm 0.9$ & $7.4 \pm 0.9$ & $<4.5$ & $<3.1$ & $84.8 \pm 7.3$ & $<-0.62$ \\
\hline J0226.5-0601 & 022629.20 & 1.13 & -060138.77 & 1.83 & $S$ & $6.4 \pm 1.2$ & $6.4 \pm 1.2$ & $<6.4$ & $<5.2$ & $17.9 \pm 33.9$ & $<-0.52$ \\
\hline J0226.5-0454 & 022630.19 & 1.18 & -045434.23 & 1.81 & $S$ & $3.6 \pm 0.7$ & $3.6 \pm 0.7$ & $<6.7$ & $<4.9$ & $52.9 \pm 20.7$ & $<-0.12$ \\
\hline J0226.5-0513 & 022632.53 & 1.06 & -051328.26 & 1.75 & $S$ & $168.3 \pm 16.9$ & $168.3 \pm 16.9$ & $2.0 \pm 0.3$ & $<1.0$ & $23.5 \pm 4.0$ & -0.48 \\
\hline J0226.6-0253 & 022632.93 & 1.16 & -025358.68 & 1.77 & $S$ & $4.3 \pm 0.7$ & $4.3 \pm 0.7$ & $<5.8$ & $<4.4$ & $93.4 \pm 19.3$ & -0.22 \\
\hline J0226.6-0515 & 022633.62 & 1.08 & -051554.65 & 1.77 & $S$ & $4.3 \pm 0.7$ & $4.3 \pm 0.7$ & $<4.0$ & $\ldots$ & $\ldots$ & $<-0.25$ \\
\hline J0226.6-0422 & 022634.03 & 1.15 & -042219.12 & 1.97 & $S$ & $2.5 \pm 0.6$ & $2.5 \pm 0.6$ & $<8.7$ & $<5.5$ & $10.7 \pm 16.5$ & 0.45 \\
\hline J0226.6-0414 & 022634.10 & 1.07 & -041435.94 & 1.75 & $S$ & $9.4 \pm 1.1$ & $9.4 \pm 1.1$ & $<3.2$ & $<2.6$ & $47.2 \pm 11.5$ & -0.44 \\
\hline J0226.6-0411 & 022635.02 & 1.12 & -041126.25 & 1.80 & $S$ & $3.7 \pm 0.6$ & $3.7 \pm 0.6$ & $<5.4$ & $<5.0$ & $-1.0 \pm 82.6$ & $<-0.14$ \\
\hline J0226.6-0512 & 022635.21 & 1.19 & -051214.60 & 1.86 & $S$ & $4.5 \pm 0.9$ & $4.5 \pm 0.9$ & $<7.9$ & $<4.7$ & $140.7 \pm 12.0$ & $<-0.27$ \\
\hline J0226.6-0432 & 022635.87 & 1.12 & -043227.58 & 1.82 & $S$ & $3.7 \pm 0.6$ & $3.7 \pm 0.6$ & $<6.0$ & $<5.0$ & $172.1 \pm 30.3$ & -0.41 \\
\hline J0226.6-0602 & 022637.35 & 1.08 & -060213.87 & 1.77 & $S$ & $18.1 \pm 2.2$ & $18.1 \pm 2.2$ & $<4.2$ & $<3.1$ & $31.2 \pm 7.7$ & -0.41 \\
\hline J0226.6-0407 & 022637.43 & 1.06 & -040759.43 & 1.75 & $S$ & $12.4 \pm 1.3$ & $12.4 \pm 1.3$ & $<2.7$ & $<2.3$ & $12.4 \pm 11.0$ & -0.37 \\
\hline J0226.6-0543 & 022637.44 & 1.29 & -054315.96 & 2.21 & $S$ & $10.0 \pm 1.9$ & $10.0 \pm 1.9$ & $<12.2$ & $<3.5$ & $152.1 \pm 2.9$ & $<-0.82$ \\
\hline J0226.6-0412 & 022637.84 & 1.12 & -041246.32 & 1.82 & $S$ & $3.4 \pm 0.6$ & $3.4 \pm 0.6$ & $<6.5$ & $<4.0$ & $31.7 \pm 10.7$ & -0.24 \\
\hline J0226.6-0500 & 022638.68 & 1.23 & -050036.12 & 2.10 & $S$ & $7.6 \pm 1.6$ & $7.6 \pm 1.6$ & $11.4 \pm 6.7$ & $<6.6$ & $12.8 \pm 13.3$ & -0.13 \\
\hline $\mathrm{J} 0226.7-0501$ & 022638.92 & 1.25 & -050155.26 & 2.39 & $S$ & $12.6 \pm 2.3$ & $12.6 \pm 2.3$ & $20.7 \pm 8.4$ & $<5.9$ & $17.0 \pm 4.9$ & -0.48 \\
\hline $\mathrm{J} 0226.7-0552 *$ & 022640.02 & 1.06 & -055236.80 & 1.75 & $S$ & $132.0 \pm 13.3$ & $132.0 \pm 13.3$ & $4.6 \pm 0.4$ & $1.9 \pm 0.6$ & $41.8 \pm 3.3$ & -0.11 \\
\hline J0226.7-0320 & 022640.94 & 1.09 & -032006.81 & 1.77 & $S$ & $6.1 \pm 0.8$ & $6.1 \pm 0.8$ & $<4.6$ & $<4.0$ & $46.8 \pm 25.4$ & -0.59 \\
\hline J0226.7-0334 & 022641.68 & 1.24 & -033424.09 & 1.79 & $S$ & $2.7 \pm 0.6$ & $2.7 \pm 0.6$ & $<7.3$ & $<4.8$ & $108.9 \pm 16.7$ & $<0.07$ \\
\hline \multirow[t]{2}{*}{ J0226.7-0446 } & 022642.23 & 1.09 & -044625.98 & 1.78 & $M$ & $9.6 \pm 1.1$ & $6.8 \pm 0.9$ & $4.6 \pm 2.9$ & $<4.1$ & $0.2 \pm 18.1$ & -0.47 \\
\hline & 022640.46 & 1.18 & -044607.12 & 1.89 & $C$ & & $2.8 \pm 0.6$ & $<7.9$ & $<5.3$ & $29.7 \pm 19.1$ & \\
\hline \multirow[t]{3}{*}{ J0226.7-0157 } & 022642.65 & 1.08 & -015732.50 & 1.77 & $M$ & $71.0 \pm 7.5$ & $31.2 \pm 3.6$ & $6.4 \pm 1.9$ & $<3.1$ & $34.5 \pm 6.2$ & -0.18 \\
\hline & 022641.32 & 1.39 & -015740.31 & 2.15 & $C$ & & $30.9 \pm 6.3$ & $13.9 \pm 6.6$ & $10.1 \pm 5.3$ & $4.1 \pm 29.4$ & \\
\hline & 022642.14 & 1.21 & -015740.27 & 1.82 & $C$ & & $9.0 \pm 1.6$ & $<6.8$ & $<6.1$ & $107.3 \pm 61.8$ & \\
\hline J0226.7-0323 & 022643.98 & 1.12 & -032355.08 & 1.79 & $S$ & $5.0 \pm 0.8$ & $5.0 \pm 0.8$ & $<5.2$ & $\ldots$ & $\ldots$ & -0.13 \\
\hline J0226.7-0206 & 022644.72 & 1.10 & -020615.78 & 1.91 & $S$ & $6.1 \pm 1.1$ & $6.1 \pm 1.1$ & $<7.9$ & $<4.6$ & $7.5 \pm 9.6$ & -0.23 \\
\hline J0226.8-0230 & 022645.81 & 1.08 & -023028.27 & 1.77 & $S$ & $4.0 \pm 0.6$ & $4.0 \pm 0.6$ & $<4.4$ & $<3.7$ & $0.7 \pm 36.0$ & $<-0.20$ \\
\hline J0226.8-0249 & 022645.91 & 1.07 & -024951.75 & 1.76 & $S$ & $10.0 \pm 1.1$ & $10.0 \pm 1.1$ & $<3.4$ & $<3.1$ & $80.4 \pm 24.4$ & -0.79 \\
\hline J0226.8-0223 & 022647.27 & 1.20 & -022335.55 & 1.85 & $S$ & $4.0 \pm 0.8$ & $4.0 \pm 0.8$ & $<7.5$ & $<5.3$ & $43.7 \pm 20.8$ & $<-0.20$ \\
\hline J0226.8-0411 & 022649.11 & 1.19 & -041113.13 & 1.80 & $M$ & $6.3 \pm 0.7$ & $3.4 \pm 0.6$ & $<7.2$ & $<2.9$ & $52.1 \pm 4.0$ & -0.66 \\
\hline & 022649.00 & 1.13 & -041110.62 & 1.77 & $C$ & & $2.9 \pm 0.5$ & $<5.9$ & $<3.3$ & $57.2 \pm 9.0$ & \\
\hline J0226.8-0232 & 022650.13 & 1.06 & -023224.52 & 1.75 & $S$ & $28.5 \pm 2.9$ & $28.5 \pm 2.9$ & $<2.3$ & $<1.8$ & $48.5 \pm 3.4$ & -1.03 \\
\hline J0226.8-0257 & 022650.33 & 1.14 & -025752.08 & 1.78 & $S$ & $3.5 \pm 0.6$ & $3.5 \pm 0.6$ & $<5.7$ & $<4.6$ & $107.1 \pm 32.2$ & $<-0.10$ \\
\hline J0226.8-0351 & 022650.37 & 1.62 & -035129.93 & 1.82 & $S$ & $5.8 \pm 1.2$ & $5.8 \pm 1.2$ & $<10.9$ & $<4.8$ & $71.7 \pm 6.4$ & $<-0.45$ \\
\hline J0226.8-0255 & 022650.37 & 1.15 & -025526.52 & 1.83 & $S$ & $3.0 \pm 0.6$ & $3.0 \pm 0.6$ & $<6.4$ & $<5.6$ & $-1.0 \pm 66.9$ & $<-0.00$ \\
\hline J0226.9-0359 & 022652.20 & 1.08 & -035921.65 & 1.77 & $S$ & $7.1 \pm 0.9$ & $7.1 \pm 0.9$ & $<4.3$ & $<3.6$ & $39.5 \pm 17.5$ & -0.57 \\
\hline J0226.9-0431 & 022652.42 & 1.10 & -043146.22 & 1.78 & $S$ & $4.8 \pm 0.7$ & $4.8 \pm 0.7$ & $<5.3$ & $<4.1$ & $141.8 \pm 15.7$ & -0.47 \\
\hline J0226.9-0301 & 022653.41 & 1.08 & -030145.09 & 1.77 & $M$ & $8.0 \pm 0.8$ & $4.3 \pm 0.6$ & $<4.5$ & $<3.3$ & $25.1 \pm 14.0$ & -0.85 \\
\hline & 022653.55 & 1.08 & -030147.69 & 1.76 & $C$ & & $3.8 \pm 0.6$ & $<4.4$ & $<3.3$ & $47.1 \pm 18.1$ & \\
\hline J0226.9-0418 & 022655.25 & 1.24 & -041810.74 & 1.82 & $S$ & $2.5 \pm 0.6$ & $2.5 \pm 0.6$ & $<7.2$ & $<6.1$ & $78.2 \pm 50.7$ & $<0.12$ \\
\hline J0226.9-0305 & 022655.75 & 1.14 & -030534.68 & 1.81 & $S$ & $3.3 \pm 0.6$ & $3.3 \pm 0.6$ & $<6.4$ & $<4.6$ & $39.8 \pm 20.5$ & $<-0.06$ \\
\hline J0226.9-0542 & 022655.84 & 1.27 & -054241.94 & 1.81 & $S$ & $8.4 \pm 1.5$ & $8.4 \pm 1.5$ & $7.1 \pm 5.0$ & $<5.6$ & $70.3 \pm 18.2$ & -0.97 \\
\hline J0226.9-0403 & 022656.32 & 1.13 & -040326.40 & 1.85 & $S$ & $3.1 \pm 0.6$ & $3.1 \pm 0.6$ & $<7.1$ & $<4.9$ & $156.6 \pm 16.9$ & $<-0.01$ \\
\hline J0227.0-0223* & 022657.37 & 1.06 & -022401.78 & 1.75 & $M$ & $42.1 \pm 3.5$ & $27.0 \pm 2.8$ & $2.4 \pm 1.4$ & $<2.2$ & $101.2 \pm 8.7$ & -0.60 \\
\hline & 022656.29 & 1.18 & -022340.35 & 2.03 & $C$ & & $9.7 \pm 1.9$ & $10.0 \pm 6.4$ & $<5.4$ & $161.0 \pm 8.7$ & \\
\hline & 022656.79 & 1.09 & -022350.19 & 1.79 & $C$ & & $5.3 \pm 0.9$ & $<5.2$ & $<4.0$ & $4.4 \pm 20.3$ & \\
\hline J0227.0-0337 & 022658.90 & 1.09 & -033740.24 & 1.77 & $S$ & $6.4 \pm 0.9$ & $6.4 \pm 0.9$ & $<4.4$ & $<4.2$ & $-1.0 \pm 82.1$ & -0.69 \\
\hline J0227.0-0500 & 022659.18 & 1.08 & -050052.42 & 1.77 & $S$ & $4.7 \pm 0.7$ & $4.7 \pm 0.7$ & $<4.3$ & $<3.7$ & $0.9 \pm 26.2$ & $<-0.31$ \\
\hline $\mathrm{J} 0227.0-0555^{*}$ & 022659.33 & 1.06 & -055548.43 & 1.75 & $M$ & $1017.2 \pm 67.7$ & $506.4 \pm 51.0$ & $19.1 \pm 0.7$ & $<1.3$ & $150.1 \pm 0.3$ & -0.93 \\
\hline & 022659.15 & 1.06 & -055540.42 & 1.75 & $C$ & & $442.1 \pm 44.3$ & $3.2 \pm 0.2$ & $<1.0$ & $42.2 \pm 1.9$ & \\
\hline & 022659.32 & 1.06 & -055534.50 & 1.76 & $C$ & & $18.5 \pm 2.2$ & $<3.2$ & $<1.9$ & $171.1 \pm 4.8$ & \\
\hline & 022659.51 & 1.09 & -055534.48 & 1.86 & $C$ & & $16.9 \pm 2.6$ & $<7.2$ & $<3.0$ & $159.4 \pm 3.7$ & \\
\hline & 022659.68 & 1.07 & -055545.18 & 1.77 & $C$ & & $16.8 \pm 2.3$ & $<4.3$ & $<2.8$ & $6.1 \pm 9.3$ & \\
\hline & 022659.79 & 1.09 & -055548.51 & 1.75 & $C$ & & $16.5 \pm 2.3$ & $<4.3$ & $<2.9$ & $98.0 \pm 9.5$ & \\
\hline J0227.0-0228 & 022659.60 & 1.15 & -022815.75 & 1.83 & $S$ & $5.2 \pm 1.0$ & $5.2 \pm 1.0$ & $<6.3$ & $<5.6$ & $14.7 \pm 50.9$ & -0.06 \\
\hline J0227.0-0328 & 022659.67 & 1.06 & -032820.66 & 1.75 & $M$ & $55.8 \pm 3.8$ & $27.7 \pm 2.9$ & $4.1 \pm 0.9$ & $<1.9$ & $44.8 \pm 3.2$ & -0.73 \\
\hline
\end{tabular}


Table A.1. continued.

\begin{tabular}{|c|c|c|c|c|c|c|c|c|c|c|c|}
\hline Name & $\begin{array}{c}\text { RA } \\
\text { J2000 }\end{array}$ & $\begin{array}{c}\sigma_{\alpha} \\
\operatorname{arcsec}\end{array}$ & $\begin{array}{c}\text { Dec } \\
\text { J2000 }\end{array}$ & $\begin{array}{c}\sigma_{\delta} \\
\operatorname{arcsec}\end{array}$ & Type & $\begin{array}{l}S_{\text {int }} \\
\text { mJy }\end{array}$ & $\begin{array}{c}S_{\text {comp }} \\
\text { mJy }\end{array}$ & $\begin{array}{c}\text { Major axis } \\
\text { arcsec }\end{array}$ & $\begin{array}{c}\text { Minor axis } \\
\operatorname{arcsec}\end{array}$ & $\begin{array}{c}\text { Position } \\
\text { Angle }\end{array}$ & $\begin{array}{c}\text { Spectral } \\
\text { index } \alpha_{325}^{1400}\end{array}$ \\
\hline & 022658.77 & 1.06 & -032848.46 & 1.75 & $C$ & & $22.6 \pm 2.4$ & $4.5 \pm 1.1$ & $<2.1$ & $32.4 \pm 3.1$ & \\
\hline & 022659.33 & 1.08 & -032827.12 & 1.77 & $C$ & & $5.5 \pm 0.7$ & $<4.7$ & $<2.9$ & $151.3 \pm 6.9$ & \\
\hline J0227.0-0539 & 022700.29 & 1.52 & -053953.21 & 1.89 & $S$ & $5.4 \pm 1.2$ & $5.4 \pm 1.2$ & $<10.3$ & $<6.4$ & $63.9 \pm 15.2$ & 0.29 \\
\hline J0227.0-0541 & 022700.93 & 1.06 & -054149.95 & 1.75 & $S$ & $36.8 \pm 3.9$ & $36.8 \pm 3.9$ & $3.3 \pm 1.2$ & $<2.5$ & $13.2 \pm 12.7$ & -0.79 \\
\hline J0227.1-0423 & 022704.18 & 1.17 & -042338.20 & 1.86 & $S$ & $2.8 \pm 0.5$ & $2.8 \pm 0.5$ & $<7.8$ & $<4.2$ & $37.3 \pm 8.7$ & $<0.04$ \\
\hline J0227.1-0543 & 022706.76 & 1.62 & -054341.29 & 1.83 & $S$ & $6.0 \pm 1.3$ & $6.0 \pm 1.3$ & $<10.7$ & $<6.0$ & $78.0 \pm 11.0$ & -0.08 \\
\hline J0227.1-0221 & 022708.21 & 1.43 & -022121.40 & 1.88 & $S$ & $4.5 \pm 1.0$ & $4.5 \pm 1.0$ & $<9.6$ & $<6.0$ & $120.6 \pm 14.8$ & $<-0.28$ \\
\hline J0227.1-0320 & 022708.81 & 1.38 & -032049.56 & 2.00 & $S$ & $7.2 \pm 1.6$ & $7.2 \pm 1.6$ & $9.5 \pm 7.1$ & $<7.0$ & $40.7 \pm 18.5$ & -0.07 \\
\hline J0227.2-0259 & 022710.94 & 1.14 & -025910.95 & 1.87 & $S$ & $4.7 \pm 0.8$ & $4.7 \pm 0.8$ & $6.6 \pm 5.4$ & $<4.2$ & $152.1 \pm 7.1$ & $<-0.31$ \\
\hline \multirow[t]{2}{*}{ J0227.2-0418 } & 022712.61 & 1.13 & -041817.15 & 1.76 & $M$ & $6.8 \pm 0.7$ & $3.6 \pm 0.5$ & $<5.4$ & $<2.9$ & $81.0 \pm 6.1$ & $<-0.56$ \\
\hline & 022712.63 & 1.10 & -041819.96 & 1.76 & $C$ & & $3.2 \pm 0.5$ & $<4.6$ & $<2.7$ & $68.2 \pm 7.0$ & \\
\hline J0227.2-0446 & 022712.94 & 1.07 & -044635.37 & 1.75 & $S$ & $12.4 \pm 1.4$ & $12.4 \pm 1.4$ & $<3.1$ & $<2.7$ & $46.4 \pm 13.6$ & -0.20 \\
\hline J0227.2-0312 & 022713.30 & 1.13 & -031245.99 & 1.78 & $S$ & $5.4 \pm 0.9$ & $5.4 \pm 0.9$ & $<5.4$ & $<4.9$ & $108.4 \pm 57.1$ & $<-0.40$ \\
\hline J0227.2-0411 & 022714.16 & 1.13 & -041128.46 & 1.93 & $S$ & $2.6 \pm 0.6$ & $2.6 \pm 0.6$ & $<8.1$ & $<5.3$ & $171.6 \pm 16.5$ & $<0.09$ \\
\hline J0227.3-0549 & 022715.32 & 1.27 & -054958.20 & 1.82 & $S$ & $6.3 \pm 1.4$ & $6.3 \pm 1.4$ & $<7.5$ & $<5.9$ & $80.8 \pm 34.3$ & -0.48 \\
\hline \multirow[t]{2}{*}{$\mathrm{J} 0227.3-0257$} & 022716.12 & 1.14 & -025734.73 & 1.76 & $M$ & $9.2 \pm 1.0$ & $5.6 \pm 0.8$ & $<5.6$ & $<2.6$ & $76.5 \pm 4.0$ & $<-0.76$ \\
\hline & 022716.11 & 1.26 & -025732.00 & 1.75 & $C$ & & $3.6 \pm 0.6$ & $<7.3$ & $<2.6$ & $83.7 \pm 3.4$ & \\
\hline \multirow[t]{6}{*}{$\mathrm{J} 0227.2-0325^{*}$} & 022716.28 & 1.17 & -032536.66 & 1.77 & $M$ & $195.8 \pm 24.1^{*}$ & $24.3 \pm 3.1$ & $10.6 \pm 2.9$ & $<3.9$ & $68.5 \pm 5.1$ & -0.20 \\
\hline & 022715.51 & 1.24 & -032537.70 & 2.69 & $C$ & & $8.4 \pm 2.3$ & $<15.2$ & $<5.8$ & $12.7 \pm 7.2$ & \\
\hline & 022715.86 & 1.66 & -032543.30 & 2.41 & $C$ & & $4.0 \pm 1.0$ & $<14.9$ & $<5.7$ & $143.0 \pm 7.0$ & \\
\hline & 022708.89 & 1.42 & -032558.35 & 2.16 & $M$ & & $32.7 \pm 6.2$ & $16.6 \pm 6.6$ & $11.9 \pm 5.0$ & $14.6 \pm 23.6$ & -0.20 \\
\hline & 022708.02 & 1.10 & -032600.32 & 1.77 & $C$ & & $22.3 \pm 2.7$ & $6.7 \pm 2.1$ & $<3.7$ & $53.3 \pm 9.8$ & \\
\hline & 022708.40 & 1.98 & -032545.81 & 2.17 & $C$ & & $9.7 \pm 2.6$ & $13.2 \pm 10.4$ & $<9.5$ & $119.6 \pm 23.0$ & \\
\hline J0227.3-0341 & 022717.06 & 1.07 & -034107.77 & 1.75 & $S$ & $38.1 \pm 4.0$ & $38.1 \pm 4.0$ & $8.3 \pm 1.0$ & $<2.0$ & $91.6 \pm 1.7$ & -0.65 \\
\hline J0227.3-0311 & 022719.86 & 1.16 & -031128.34 & 1.78 & $S$ & $4.7 \pm 0.8$ & $4.7 \pm 0.8$ & $<6.0$ & $<4.3$ & $69.4 \pm 15.4$ & $<-0.30$ \\
\hline $\mathrm{J} 0227.3-0330$ & 022720.51 & 1.06 & -033036.65 & 1.75 & $S$ & $14.4 \pm 1.5$ & $14.4 \pm 1.5$ & $<2.7$ & $<2.3$ & $44.9 \pm 12.2$ & -1.29 \\
\hline J0227.3-0445 & 022720.67 & 1.11 & -044535.40 & 1.79 & $S$ & $5.3 \pm 0.8$ & $5.3 \pm 0.8$ & $<5.3$ & $<4.8$ & $28.7 \pm 45.8$ & $<-0.39$ \\
\hline J0227.4-0341 & 022722.42 & 1.12 & -034122.27 & 1.77 & $S$ & $5.3 \pm 0.8$ & $5.3 \pm 0.8$ & $<5.4$ & $<3.9$ & $59.7 \pm 14.0$ & -0.51 \\
\hline J0227.4-0433 & 022722.73 & 1.10 & -043350.03 & 1.78 & $S$ & $6.4 \pm 0.9$ & $6.4 \pm 0.9$ & $<5.0$ & $<4.3$ & $35.6 \pm 25.9$ & $<-0.52$ \\
\hline \multirow[t]{4}{*}{ J0227.4-0240* } & 022722.89 & 1.08 & -023953.10 & 1.76 & $M$ & $119.7 \pm 7.2$ & $43.1 \pm 4.6$ & $9.8 \pm 1.3$ & $<2.5$ & $116.3 \pm 2.4$ & -0.76 \\
\hline & 022726.52 & 1.06 & -024019.50 & 1.75 & $C$ & & $35.4 \pm 3.6$ & $<2.3$ & $<2.1$ & $78.4 \pm 12.6$ & \\
\hline & 022725.94 & 1.84 & -024018.91 & 2.30 & $C$ & & $22.4 \pm 3.3$ & $25.3 \pm 10.4$ & $<2.6$ & $45.1 \pm 0.8$ & \\
\hline & 022725.62 & 1.23 & -024018.22 & 1.78 & $C$ & & $18.7 \pm 2.6$ & $11.3 \pm 3.5$ & $4.5 \pm 2.7$ & $105.6 \pm 7.4$ & \\
\hline J0227.4-0512 & 022723.06 & 1.41 & -051238.29 & 2.00 & $S$ & $3.6 \pm 0.8$ & $3.6 \pm 0.8$ & $<9.9$ & $<7.6$ & $41.9 \pm 29.4$ & -0.10 \\
\hline \multirow[t]{2}{*}{$\mathrm{J} 0227.4-0254$} & 022723.36 & 1.07 & -025424.53 & 1.75 & $M$ & $21.6 \pm 1.7$ & $11.8 \pm 1.3$ & $<3.0$ & $<1.8$ & $80.0 \pm 3.2$ & -1.05 \\
\hline & 022723.37 & 1.07 & -025427.49 & 1.75 & $C$ & & $9.9 \pm 1.1$ & $<3.5$ & $<1.7$ & $71.7 \pm 2.2$ & \\
\hline J0227.4-0241 & 022723.37 & 1.10 & -024139.35 & 1.75 & $S$ & $7.0 \pm 0.8$ & $7.0 \pm 0.8$ & $<4.4$ & $<1.6$ & $92.1 \pm 1.7$ & $<-0.58$ \\
\hline \multirow[t]{2}{*}{ J0227.4-0223 } & 022725.41 & 1.07 & -022349.50 & 1.75 & $M$ & $22.9 \pm 2.1$ & $16.2 \pm 1.8$ & $<3.1$ & $<2.8$ & $44.6 \pm 23.1$ & -0.64 \\
\hline & 022724.85 & 1.10 & -022333.07 & 1.78 & $C$ & & $6.6 \pm 1.0$ & $<5.0$ & $<3.9$ & $41.5 \pm 21.1$ & \\
\hline J0227.4-0538 & 022726.87 & 1.22 & -053843.83 & 1.92 & $S$ & $4.4 \pm 1.0$ & $4.4 \pm 1.0$ & $<8.4$ & $<6.2$ & $151.1 \pm 26.7$ & $<-0.25$ \\
\hline J0227.5-0437* & 022727.85 & 1.06 & -043733.65 & 1.75 & $M$ & $67.3 \pm 6.5$ & $64.1 \pm 6.4$ & $2.8 \pm 0.4$ & $<1.4$ & $176.6 \pm 6.6$ & -0.80 \\
\hline & 022728.21 & 1.14 & -043726.76 & 1.94 & $C$ & & $3.3 \pm 0.7$ & $<8.3$ & $<5.1$ & $165.6 \pm 13.5$ & \\
\hline J0227.5-0403 & 022728.01 & 1.06 & -040338.84 & 1.75 & $M$ & $23.6 \pm 1.8$ & $13.3 \pm 1.4$ & $<3.0$ & $<2.5$ & $159.2 \pm 8.8$ & -1.20 \\
\hline & 022728.50 & 1.08 & -040346.45 & 1.76 & $C$ & & $7.2 \pm 0.9$ & $<3.7$ & $<3.1$ & $47.5 \pm 16.0$ & \\
\hline & 022728.54 & 1.13 & -040349.39 & 1.77 & $C$ & & $3.0 \pm 0.5$ & $<5.6$ & $<3.3$ & $64.7 \pm 9.5$ & \\
\hline J0227.5-0402 & 022730.38 & 1.10 & -040217.54 & 1.79 & $S$ & $5.1 \pm 0.8$ & $5.1 \pm 0.8$ & $<5.3$ & $<4.3$ & $10.7 \pm 21.1$ & -0.63 \\
\hline J0227.5-0519 & 022731.17 & 1.11 & -051915.00 & 1.78 & $S$ & $7.1 \pm 1.0$ & $7.1 \pm 1.0$ & $<5.5$ & $<3.8$ & $45.4 \pm 9.4$ & -0.26 \\
\hline J0227.5-0524 & 022731.86 & 1.29 & -052411.32 & 1.83 & $S$ & $3.8 \pm 0.8$ & $3.8 \pm 0.8$ & $<8.0$ & $<5.8$ & $115.9 \pm 22.8$ & $<-0.17$ \\
\hline J0227.5-0201 & 022731.93 & 1.48 & -020148.15 & 1.92 & $S$ & $11.2 \pm 2.2$ & $11.2 \pm 2.2$ & $9.8 \pm 7.8$ & $<5.0$ & $53.4 \pm 6.7$ & $<-0.90$ \\
\hline J0227.5-0327 & 022732.24 & 1.14 & -032735.30 & 1.79 & $S$ & $4.4 \pm 0.8$ & $4.4 \pm 0.8$ & $<5.8$ & $<4.7$ & $57.6 \pm 29.0$ & 0.28 \\
\hline J0227.5-0557* & 022732.95 & 1.07 & -055747.96 & 1.76 & $M$ & $114.4 \pm 7.9$ & $36.7 \pm 4.0$ & $3.6 \pm 1.7$ & $<2.8$ & $21.1 \pm 9.6$ & -0.39 \\
\hline & 022731.75 & 1.23 & -055748.65 & 1.79 & $C$ & & $30.7 \pm 4.3$ & $11.5 \pm 3.6$ & $4.9 \pm 2.6$ & $107.1 \pm 7.6$ & \\
\hline & 022730.78 & 1.46 & -055749.00 & 1.81 & $C$ & & $25.9 \pm 4.3$ & $14.0 \pm 5.3$ & $5.4 \pm 3.5$ & $97.2 \pm 8.1$ & \\
\hline & 022732.65 & 1.08 & -055745.42 & 1.82 & $C$ & & $21.1 \pm 3.0$ & $6.3 \pm 3.5$ & $<3.8$ & $0.7 \pm 7.5$ & \\
\hline J0227.6-0433 & 022733.93 & 1.14 & -043312.90 & 1.78 & $S$ & $4.2 \pm 0.7$ & $4.2 \pm 0.7$ & $<5.9$ & $<4.2$ & $55.7 \pm 16.6$ & -0.09 \\
\hline J0227.6-0456 & 022734.37 & 1.06 & -045649.31 & 2.04 & $M$ & $11.5 \pm 1.1$ & $8.4 \pm 0.9$ & $9.5 \pm 6.4$ & $<0.4$ & $179.7 \pm 0.0$ & $<-0.92$ \\
\hline & 022734.04 & 1.31 & -045652.42 & 1.99 & $C$ & & $3.1 \pm 0.7$ & $<10.3$ & $<4.3$ & $38.7 \pm 5.9$ & \\
\hline J0227.6-0435 & 022735.47 & 1.11 & -043553.13 & 1.77 & $S$ & $4.8 \pm 0.7$ & $4.8 \pm 0.7$ & $<4.8$ & $<4.2$ & $71.6 \pm 39.6$ & $<-0.33$ \\
\hline J0227.6-0314* & 022735.89 & 1.06 & -031437.39 & 1.75 & $M$ & $83.7 \pm 6.6$ & $62.5 \pm 6.3$ & $4.2 \pm 0.5$ & $<1.3$ & $32.4 \pm 1.3$ & -1.14 \\
\hline & 022735.67 & 1.06 & -031437.98 & 1.75 & $C$ & & $17.5 \pm 1.8$ & $<2.1$ & $<0.4$ & $87.8 \pm 0.1$ & \\
\hline & 022732.84 & 1.55 & -031455.51 & 1.86 & $C$ & & $3.7 \pm 0.8$ & $<10.2$ & $<6.2$ & $109.0 \pm 13.7$ & \\
\hline J0227.5-0411 & 022737.23 & 1.07 & -041106.70 & 1.76 & $M$ & $46.3 \pm 2.8$ & $10.8 \pm 1.2$ & $<3.5$ & $<3.1$ & $150.7 \pm 18.3$ & -0.93 \\
\hline & 022736.86 & 1.09 & -041107.33 & 1.87 & $C$ & & $10.8 \pm 1.6$ & $10.2 \pm 3.8$ & $<4.3$ & $176.3 \pm 6.2$ & \\
\hline & 022734.18 & 1.12 & -041140.36 & 1.80 & $C$ & & $9.3 \pm 1.3$ & $7.3 \pm 3.3$ & $<4.3$ & $39.2 \pm 9.9$ & \\
\hline & 022730.48 & 1.07 & -041117.13 & 1.76 & $C$ & & $8.8 \pm 1.0$ & $<3.5$ & $<3.2$ & $16.0 \pm 27.2$ & \\
\hline & 022733.87 & 1.25 & -041143.36 & 1.89 & $C$ & & $3.3 \pm 0.7$ & $<8.7$ & $<4.6$ & $137.7 \pm 8.9$ & \\
\hline & 022736.43 & 1.15 & -041108.76 & 1.88 & $C$ & & $3.3 \pm 0.7$ & $<7.3$ & $<5.8$ & $4.4 \pm 31.1$ & \\
\hline J0227.7-0254 & 022743.00 & 1.35 & -025402.71 & 1.81 & $S$ & $4.7 \pm 0.9$ & $4.7 \pm 0.9$ & $<8.5$ & $<5.2$ & $72.9 \pm 12.0$ & -0.44 \\
\hline $\mathrm{J} 0227.7-0502$ & 022743.63 & 1.06 & -050250.96 & 1.75 & $S$ & $24.0 \pm 2.5$ & $24.0 \pm 2.5$ & $3.6 \pm 1.1$ & $<2.4$ & $138.1 \pm 10.5$ & -0.48 \\
\hline J0227.7-0250* & 022744.16 & 1.06 & -025045.73 & 1.75 & $M$ & $137.7 \pm 11.5$ & $112.0 \pm 11.2$ & $3.4 \pm 0.3$ & $1.7 \pm 0.5$ & $123.3 \pm 4.4$ & -0.76 \\
\hline & 022744.93 & 1.07 & -025100.49 & 1.75 & $C$ & & $17.6 \pm 1.9$ & $<3.2$ & $<2.5$ & $98.1 \pm 6.9$ & \\
\hline & 022744.91 & 1.10 & -025103.48 & 1.75 & $C$ & & $8.1 \pm 1.0$ & $<4.5$ & $<2.5$ & $97.3 \pm 4.6$ & \\
\hline J0227.8-0315 & 022745.22 & 1.07 & -031546.99 & 1.75 & $S$ & $17.5 \pm 1.9$ & $17.5 \pm 1.9$ & $<2.8$ & $<2.6$ & $50.2 \pm 24.8$ & -0.73 \\
\hline J0227.8-0202 & 022745.82 & 1.30 & -020300.14 & 1.91 & $S$ & $21.2 \pm 3.3$ & $21.2 \pm 3.3$ & $13.0 \pm 5.6$ & $<4.0$ & $135.5 \pm 3.7$ & -0.31 \\
\hline
\end{tabular}


C. Tasse et al.: The low-frequency counterpart of the XMM large scale structure survey, Online Material p 20

Table A.1. continued.

\begin{tabular}{|c|c|c|c|c|c|c|c|c|c|c|c|}
\hline Name & $\begin{array}{c}\text { RA } \\
\text { J2000 }\end{array}$ & $\begin{array}{c}\sigma_{\alpha} \\
\operatorname{arcsec}\end{array}$ & $\begin{array}{c}\text { Dec } \\
\text { J2000 }\end{array}$ & $\begin{array}{c}\sigma_{\delta} \\
\operatorname{arcsec}\end{array}$ & Type & $\begin{array}{l}S_{\text {int }} \\
\text { mJy }\end{array}$ & $\begin{array}{c}S_{\text {comp }} \\
\text { mJy }\end{array}$ & $\begin{array}{c}\text { Major axis } \\
\text { arcsec }\end{array}$ & $\begin{array}{c}\text { Minor axis } \\
\text { arcsec }\end{array}$ & $\begin{array}{c}\text { Position } \\
\text { Angle }\end{array}$ & $\begin{array}{c}\text { Spectral } \\
\text { index } \alpha_{325}^{1400}\end{array}$ \\
\hline J0227.8-0552 & 022746.97 & 1.20 & -055258.10 & 1.84 & $\bar{S}$ & $5.9 \pm 1.2$ & $5.9 \pm 1.2$ & $<7.5$ & $<5.1$ & $43.4 \pm 19.0$ & $<-0.46$ \\
\hline J0227.8-0232 & 022748.26 & 1.06 & -023207.08 & 1.75 & $S$ & $30.6 \pm 3.2$ & $30.6 \pm 3.2$ & $2.5 \pm 1.3$ & $<2.1$ & $62.2 \pm 5.2$ & -0.46 \\
\hline J0227.9-0518 & 022750.91 & 1.15 & -051856.64 & 1.94 & $S$ & $4.2 \pm 0.8$ & $4.2 \pm 0.8$ & $<8.3$ & $<5.6$ & $171.9 \pm 15.6$ & $<-0.23$ \\
\hline J0227.9-0429 & 022752.80 & 1.07 & -042940.69 & 1.76 & $S$ & $8.0 \pm 1.0$ & $8.0 \pm 1.0$ & $<3.8$ & $<3.3$ & $33.1 \pm 23.0$ & $<-0.67$ \\
\hline \multirow[t]{2}{*}{ J0227.9-0343 } & 022753.53 & 1.09 & -034352.70 & 1.77 & $M$ & $19.3 \pm 1.9$ & $13.3 \pm 1.6$ & $5.4 \pm 2.6$ & $<3.1$ & $37.5 \pm 5.1$ & -0.82 \\
\hline & 022753.31 & 1.10 & -034354.23 & 1.78 & $C$ & & $6.0 \pm 0.9$ & $<5.4$ & $<3.8$ & $141.0 \pm 12.0$ & \\
\hline \multirow[t]{2}{*}{ J0227.9-0444 } & 022753.91 & 1.07 & -044450.48 & 1.77 & $M$ & $35.8 \pm 3.5$ & $30.2 \pm 3.4$ & $8.4 \pm 1.5$ & $4.7 \pm 1.3$ & $160.2 \pm 6.5$ & -0.78 \\
\hline & 022754.44 & 1.08 & -044457.98 & 1.85 & $C$ & & $5.6 \pm 0.9$ & $<6.8$ & $<3.7$ & $4.3 \pm 6.3$ & \\
\hline J0227.9-0233 & 022754.14 & 1.09 & -023348.43 & 1.77 & $S$ & $11.3 \pm 1.5$ & $11.3 \pm 1.5$ & $<4.6$ & $<3.8$ & $45.5 \pm 18.9$ & -0.40 \\
\hline J0227.9-0457 & 022754.79 & 1.06 & -045703.22 & 1.75 & $S$ & $61.1 \pm 6.2$ & $61.1 \pm 6.2$ & $1.9 \pm 0.7$ & $<1.5$ & $50.0 \pm 7.2$ & -0.28 \\
\hline J0228.0-0400 & 022759.93 & 1.17 & -040015.18 & 1.88 & $S$ & $8.5 \pm 1.4$ & $8.5 \pm 1.4$ & $9.8 \pm 4.5$ & $<4.8$ & $32.6 \pm 8.1$ & -0.50 \\
\hline \multirow[t]{2}{*}{ J0228.0-0325 } & 022800.06 & 1.11 & -032547.13 & 1.77 & $M$ & $11.3 \pm 1.1$ & $6.6 \pm 0.9$ & $<5.2$ & $<3.0$ & $58.6 \pm 5.8$ & $<-0.91$ \\
\hline & 022800.14 & 1.11 & -032549.76 & 1.76 & $C$ & & $4.7 \pm 0.7$ & $<5.0$ & $<2.5$ & $69.2 \pm 5.1$ & \\
\hline J0228.0-0312 & 022801.94 & 1.09 & -031213.31 & 1.76 & $S$ & $6.2 \pm 0.9$ & $6.2 \pm 0.9$ & $<4.7$ & $<3.2$ & $51.5 \pm 10.1$ & $<-0.50$ \\
\hline \multirow[t]{2}{*}{$\mathrm{J} 0228.1-0505$} & 022805.42 & 1.08 & -050547.19 & 1.78 & $M$ & $9.5 \pm 1.1$ & $6.1 \pm 0.9$ & $<4.9$ & $<3.3$ & $162.7 \pm 9.6$ & $<-0.79$ \\
\hline & 022805.60 & 1.26 & -050546.15 & 2.01 & $C$ & & $3.4 \pm 0.6$ & $<10.3$ & $<3.1$ & $34.4 \pm 3.2$ & \\
\hline J0228.1-0242 & 022807.53 & 1.35 & -024204.79 & 1.90 & $S$ & $11.7 \pm 2.3$ & $11.7 \pm 2.3$ & $9.1 \pm 5.1$ & $7.7 \pm 4.8$ & $74.5 \pm 73.2$ & $<-0.93$ \\
\hline J0228.1-0231* & 022807.58 & 1.06 & -023106.85 & 1.75 & $S$ & $531.1 \pm 53.2$ & $531.1 \pm 53.2$ & $8.1 \pm 0.1$ & $1.2 \pm 0.3$ & $114.0 \pm 0.3$ & -0.99 \\
\hline \multirow[t]{2}{*}{$\mathrm{J} 0228.1-0408$} & 022808.46 & 1.07 & -040851.21 & 1.75 & $M$ & $15.5 \pm 1.4$ & $11.9 \pm 1.3$ & $<2.9$ & $<2.8$ & $41.5 \pm 32.0$ & -0.82 \\
\hline & 022811.29 & 1.11 & -040842.76 & 1.77 & $C$ & & $3.6 \pm 0.6$ & $<5.2$ & $<3.6$ & $63.4 \pm 15.4$ & \\
\hline J0228.2-0232 & 022809.89 & 1.12 & -023208.30 & 1.76 & $S$ & $8.6 \pm 1.2$ & $8.6 \pm 1.2$ & $<5.4$ & $<2.6$ & $120.7 \pm 4.8$ & $<-0.72$ \\
\hline J0228.2-0229 & 022810.26 & 1.43 & -022949.25 & 1.80 & $S$ & $6.9 \pm 1.3$ & $6.9 \pm 1.3$ & $<9.4$ & $<3.8$ & $110.3 \pm 5.3$ & $<-0.57$ \\
\hline J0228.2-0433 & 022814.13 & 1.17 & -043332.14 & 1.81 & $S$ & $5.3 \pm 0.8$ & $5.3 \pm 0.8$ & $<7.0$ & $<3.7$ & $131.0 \pm 5.8$ & $<-0.39$ \\
\hline \multirow[t]{3}{*}{ J0228.2-0503 } & 022814.39 & 1.18 & -050240.64 & 2.06 & $M$ & $50.0 \pm 5.0$ & $30.9 \pm 4.5$ & $20.8 \pm 5.6$ & $7.2 \pm 2.6$ & $19.1 \pm 4.3$ & -0.45 \\
\hline & 022813.58 & 1.21 & -050318.43 & 1.94 & $C$ & & $10.8 \pm 1.9$ & $11.0 \pm 5.3$ & $<5.3$ & $29.6 \pm 8.7$ & \\
\hline & 022814.35 & 1.08 & -050236.60 & 1.77 & $C$ & & $8.3 \pm 1.1$ & $<4.3$ & $<3.6$ & $15.9 \pm 19.1$ & \\
\hline J0228.3-0414 & 022816.16 & 1.21 & -041355.37 & 1.80 & $M$ & $11.9 \pm 1.5$ & $8.1 \pm 1.3$ & $6.8 \pm 4.6$ & $<4.3$ & $60.3 \pm 8.2$ & -1.00 \\
\hline & 022815.34 & 1.13 & -041405.07 & 1.81 & $C$ & & $3.8 \pm 0.7$ & $<5.8$ & $<5.2$ & $-1.0 \pm 74.1$ & \\
\hline J0228.3-0422 & 022816.64 & 1.10 & -042210.75 & 1.77 & $S$ & $5.1 \pm 0.8$ & $5.1 \pm 0.8$ & $<5.0$ & $<3.8$ & $48.6 \pm 15.8$ & -0.19 \\
\hline J0228.3-0423 & 022818.88 & 1.21 & -042312.75 & 1.85 & $S$ & $3.6 \pm 0.8$ & $3.6 \pm 0.8$ & $<7.3$ & $<6.1$ & $42.8 \pm 44.2$ & $<-0.12$ \\
\hline J0228.3-0404 & 022819.08 & 1.09 & -040500.68 & 1.77 & $S$ & $7.3 \pm 1.0$ & $7.3 \pm 1.0$ & $<4.6$ & $<3.7$ & $46.4 \pm 16.0$ & $<-0.61$ \\
\hline J0228.3-0432 & 022820.65 & 1.08 & -043227.58 & 1.79 & $S$ & $5.5 \pm 0.9$ & $5.5 \pm 0.9$ & $<5.2$ & $<3.6$ & $179.4 \pm 12.6$ & $<-0.42$ \\
\hline J0228.4-0323 & 022821.59 & 1.11 & -032342.58 & 1.78 & $S$ & $7.2 \pm 1.1$ & $7.2 \pm 1.1$ & $<5.0$ & $<4.4$ & $60.1 \pm 42.5$ & $<-0.60$ \\
\hline J0228.4-0259 & 022821.84 & 1.11 & -025926.03 & 1.78 & $M$ & $11.9 \pm 1.4$ & $7.6 \pm 1.1$ & $<4.9$ & $<4.5$ & $62.0 \pm 52.3$ & -0.77 \\
\hline & 022822.03 & 1.36 & -025925.52 & 1.93 & $C$ & & $4.3 \pm 0.8$ & $<10.2$ & $<3.7$ & $46.6 \pm 4.3$ & \\
\hline J0228.4-0453 & 022822.30 & 1.22 & -045342.86 & 1.93 & $S$ & $3.8 \pm 0.8$ & $3.8 \pm 0.8$ & $<9.1$ & $<4.2$ & $35.6 \pm 7.0$ & $<-0.17$ \\
\hline J0228.4-0430 & 022823.28 & 1.09 & -043025.09 & 1.77 & $M$ & $39.6 \pm 3.8$ & $32.2 \pm 3.6$ & $10.8 \pm 2.0$ & $<2.8$ & $133.4 \pm 2.5$ & -0.74 \\
\hline & 022823.04 & 1.18 & -043009.04 & 1.79 & $C$ & & $7.4 \pm 1.2$ & $<6.4$ & $<4.7$ & $113.5 \pm 14.8$ & \\
\hline J0228.4-0427 & 022824.05 & 1.26 & -042707.86 & 1.81 & $M$ & $16.6 \pm 2.1$ & $11.8 \pm 1.9$ & $9.5 \pm 4.1$ & $5.0 \pm 3.7$ & $107.8 \pm 15.8$ & -0.45 \\
\hline & 022824.58 & 1.14 & -042710.77 & 1.92 & $C$ & & $4.9 \pm 0.9$ & $<8.4$ & $<3.8$ & $25.6 \pm 5.4$ & \\
\hline J0228.4-0349 & 022824.51 & 1.08 & -034908.53 & 1.77 & $S$ & $5.5 \pm 0.8$ & $5.5 \pm 0.8$ & $<4.2$ & $<3.7$ & $41.3 \pm 42.6$ & $<-0.42$ \\
\hline J0228.4-0435 & 022825.29 & 1.07 & -043440.25 & 1.76 & $M$ & $16.4 \pm 1.6$ & $12.5 \pm 1.4$ & $<3.2$ & $<2.9$ & $40.7 \pm 22.0$ & -0.70 \\
\hline & 022822.18 & 1.07 & -043504.12 & 2.01 & $C$ & & $4.0 \pm 0.7$ & $<9.2$ & $<3.0$ & $173.6 \pm 3.4$ & \\
\hline J0228.4-0326 & 022825.31 & 1.22 & -032638.71 & 1.81 & $S$ & $5.0 \pm 1.1$ & $5.0 \pm 1.1$ & $<6.8$ & $<5.8$ & $76.5 \pm 46.9$ & $<-0.35$ \\
\hline J0228.4-0417 & 022826.78 & 1.11 & -041751.77 & 1.78 & $S$ & $6.9 \pm 1.0$ & $6.9 \pm 1.0$ & $<5.0$ & $<4.5$ & $55.8 \pm 38.6$ & $<-0.57$ \\
\hline J0228.5-0540 & 022827.54 & 1.06 & -054100.89 & 1.75 & $S$ & $81.6 \pm 8.3$ & $81.6 \pm 8.3$ & $5.6 \pm 0.6$ & $2.7 \pm 0.7$ & $142.2 \pm 4.0$ & -0.32 \\
\hline J0228.5-0223* & 022829.19 & 1.06 & -022316.42 & 1.75 & $S$ & $172.2 \pm 17.3$ & $172.2 \pm 17.3$ & $4.1 \pm 0.3$ & $1.0 \pm 0.7$ & $100.4 \pm 2.2$ & -0.81 \\
\hline J0228.5-0416 & 022831.46 & 1.21 & -041624.36 & 1.89 & $S$ & $4.0 \pm 0.9$ & $4.0 \pm 0.9$ & $<7.4$ & $<6.6$ & $-1.0 \pm 69.0$ & $<-0.20$ \\
\hline J0228.5-0446 & 022831.62 & 1.08 & -044605.30 & 1.77 & $M$ & $21.2 \pm 2.1$ & $16.2 \pm 2.0$ & $5.6 \pm 2.2$ & $<3.1$ & $151.2 \pm 6.1$ & -0.75 \\
\hline & 022831.69 & 1.22 & -044603.65 & 1.85 & $C$ & & $5.0 \pm 0.9$ & $<8.2$ & $<3.7$ & $45.7 \pm 5.5$ & \\
\hline J0228.6-0417 & 022834.32 & 1.09 & -041738.25 & 2.00 & $S$ & $3.8 \pm 0.7$ & $3.8 \pm 0.7$ & $<9.2$ & $<3.7$ & $9.5 \pm 5.4$ & $<-0.16$ \\
\hline J0228.6-0339 & 022834.64 & 1.07 & -034000.06 & 1.76 & $S$ & $21.3 \pm 2.4$ & $21.3 \pm 2.4$ & $<3.3$ & $<3.0$ & $85.9 \pm 23.0$ & -0.46 \\
\hline J0228.6-0428 & 022835.86 & 1.16 & -042852.41 & 1.87 & $S$ & $3.8 \pm 0.9$ & $3.8 \pm 0.9$ & $<7.2$ & $<5.8$ & $164.5 \pm 39.5$ & $<-0.17$ \\
\hline J0228.6-0449 & 022836.52 & 1.23 & -044929.85 & 2.11 & $S$ & $3.7 \pm 0.9$ & $3.7 \pm 0.9$ & $<10.3$ & $<6.9$ & $1.5 \pm 18.7$ & $<-0.15$ \\
\hline J0228.6-0505 & 022838.46 & 1.12 & -050512.37 & 1.81 & $S$ & $5.8 \pm 0.9$ & $5.8 \pm 0.9$ & $<6.2$ & $<4.2$ & $33.8 \pm 11.8$ & 0.27 \\
\hline J0228.6-0406 & 022838.49 & 1.06 & -040647.77 & 1.75 & $S$ & $30.0 \pm 3.1$ & $30.0 \pm 3.1$ & $<2.4$ & $<2.2$ & $6.5 \pm 14.5$ & -0.23 \\
\hline J0228.7-0435 & 022839.30 & 1.07 & -043552.77 & 1.76 & $M$ & $36.9 \pm 2.5$ & $16.3 \pm 1.8$ & $<3.2$ & $<2.8$ & $176.9 \pm 13.9$ & -0.86 \\
\hline & 022839.66 & 1.07 & -043542.01 & 1.76 & $C$ & & $10.5 \pm 1.2$ & $<3.5$ & $<2.5$ & $20.5 \pm 8.3$ & \\
\hline & 022839.79 & 1.08 & -043544.08 & 1.77 & $C$ & & $10.2 \pm 1.2$ & $<4.8$ & $<2.0$ & $32.0 \pm 2.1$ & \\
\hline J0228.8-0502 & 022845.58 & 1.08 & -050232.17 & 1.76 & $M$ & $10.1 \pm 1.1$ & $6.5 \pm 0.9$ & $<4.3$ & $<3.2$ & $46.8 \pm 12.3$ & -0.85 \\
\hline & 022845.65 & 1.54 & -050228.92 & 1.80 & $C$ & & $3.6 \pm 0.7$ & $<10.2$ & $<3.2$ & $71.1 \pm 3.2$ & \\
\hline J0228.8-0213 & 022845.96 & 1.21 & -021327.99 & 1.79 & $S$ & $9.4 \pm 1.6$ & $9.4 \pm 1.6$ & $<7.1$ & $<3.9$ & $62.8 \pm 7.3$ & -0.67 \\
\hline J0228.8-0253 & 022847.52 & 1.08 & -025348.02 & 2.65 & $S$ & $34.7 \pm 5.3$ & $34.7 \pm 5.3$ & $25.7 \pm 9.7$ & $<3.5$ & $178.3 \pm 1.4$ & $<-1.68$ \\
\hline J0228.8-0357 & 022847.74 & 1.09 & -035732.98 & 2.11 & $S$ & $19.0 \pm 3.1$ & $19.0 \pm 3.1$ & $11.7 \pm 6.7$ & $<3.2$ & $10.2 \pm 2.3$ & $<-1.27$ \\
\hline J0228.8-0238 & 022849.27 & 1.40 & -023847.37 & 1.82 & $S$ & $6.0 \pm 1.2$ & $6.0 \pm 1.2$ & $<9.1$ & $<5.1$ & $110.3 \pm 10.9$ & -0.41 \\
\hline J0228.9-0337* & 022853.22 & 1.06 & -033736.60 & 1.75 & $M$ & $2144.5 \pm 200.4$ & $2000.5 \pm 200.1$ & $4.5 \pm 0.1$ & $2.6 \pm 0.1$ & $129.0 \pm 0.8$ & -0.54 \\
\hline & 022853.69 & 1.06 & -033741.21 & 1.75 & $C$ & & $98.9 \pm 10.3$ & $<2.6$ & $<1.7$ & $36.8 \pm 2.4$ & \\
\hline & 022853.59 & 1.07 & -033731.11 & 1.76 & $C$ & & $45.0 \pm 5.2$ & $<4.0$ & $<2.2$ & $142.6 \pm 3.4$ & \\
\hline J0228.9-0350 & 022853.47 & 1.07 & -035054.75 & 1.77 & $M$ & $28.7 \pm 3.1$ & $17.1 \pm 2.3$ & $<4.5$ & $<3.1$ & $166.3 \pm 10.0$ & -0.66 \\
\hline & 022853.65 & 1.12 & -035055.02 & 1.81 & $C$ & & $11.5 \pm 2.0$ & $<6.5$ & $<3.4$ & $38.0 \pm 7.4$ & \\
\hline J0228.9-0224 & 022855.32 & 1.09 & -022456.08 & 1.76 & $S$ & $11.9 \pm 1.6$ & $11.9 \pm 1.6$ & $<4.2$ & $<3.7$ & $55.6 \pm 27.6$ & -0.44 \\
\hline J0229.1-0436 & 022904.27 & 1.14 & -043646.66 & 1.86 & $S$ & $6.8 \pm 1.2$ & $6.8 \pm 1.2$ & $<7.6$ & $<3.4$ & $147.2 \pm 5.2$ & $<-0.56$ \\
\hline J0229.1-0421 & 022904.99 & 1.07 & -042121.15 & 1.76 & $S$ & $21.4 \pm 2.5$ & $21.4 \pm 2.5$ & $<3.1$ & $\ldots$ & $\ldots$ & -0.61 \\
\hline J0229.1-0541* & 022905.81 & 1.07 & -054106.50 & 1.75 & $M$ & $759.5 \pm 69.0$ & $681.7 \pm 68.5$ & $29.1 \pm 0.8$ & $6.9 \pm 0.2$ & $51.3 \pm 0.3$ & -0.86 \\
\hline
\end{tabular}


C. Tasse et al.: The low-frequency counterpart of the XMM large scale structure survey, Online Material p 21

Table A.1. continued.

\begin{tabular}{|c|c|c|c|c|c|c|c|c|c|c|c|}
\hline Name & $\begin{array}{c}\text { RA } \\
\text { J2000 }\end{array}$ & $\begin{array}{c}\sigma_{\alpha} \\
\operatorname{arcsec}\end{array}$ & $\begin{array}{c}\text { Dec } \\
\text { J2000 }\end{array}$ & $\begin{array}{c}\sigma_{\delta} \\
\operatorname{arcsec}\end{array}$ & Type & $\begin{array}{l}S_{\text {int }} \\
\text { mJy }\end{array}$ & $\begin{array}{c}S_{\text {comp }} \\
\text { mJy }\end{array}$ & $\begin{array}{c}\text { Major axis } \\
\text { arcsec }\end{array}$ & $\begin{array}{c}\text { Minor axis } \\
\text { arcsec }\end{array}$ & $\begin{array}{c}\text { Position } \\
\text { Angle }\end{array}$ & $\begin{array}{c}\text { Spectral } \\
\text { index } \alpha_{325}^{1400}\end{array}$ \\
\hline & 022905.19 & 1.07 & -054111.02 & 1.75 & $C$ & & $77.8 \pm 8.1$ & $6.7 \pm 0.8$ & $<2.0$ & $79.7 \pm 2.3$ & \\
\hline \multirow[t]{2}{*}{ J0229.1-0402 } & 022905.90 & 1.07 & -040241.40 & 1.75 & $M$ & $58.6 \pm 5.2$ & $47.4 \pm 5.0$ & $4.5 \pm 1.1$ & $<2.2$ & $54.9 \pm 4.4$ & -0.36 \\
\hline & 022906.38 & 1.07 & -040237.20 & 1.77 & $C$ & & $11.3 \pm 1.6$ & $<4.5$ & $<3.4$ & $176.7 \pm 14.7$ & \\
\hline J0229.1-0443 & 022906.03 & 1.09 & -044347.21 & 1.77 & $S$ & $15.7 \pm 2.0$ & $15.7 \pm 2.0$ & $<4.6$ & $<3.9$ & $34.7 \pm 21.0$ & -0.56 \\
\hline J0229.1-0510 & 022907.77 & 1.11 & -051012.57 & 1.78 & $S$ & $8.4 \pm 1.3$ & $8.4 \pm 1.3$ & $<5.2$ & $<4.4$ & $45.2 \pm 35.1$ & $<-0.71$ \\
\hline J0229.1-0508 & 022908.49 & 1.11 & -050831.07 & 1.80 & $S$ & $9.3 \pm 1.5$ & $9.3 \pm 1.5$ & $<5.6$ & $<4.5$ & $30.9 \pm 22.2$ & -0.66 \\
\hline \multirow[t]{2}{*}{ J0229.1-0507 } & 022908.69 & 1.07 & -050724.31 & 1.76 & $M$ & $32.8 \pm 3.2$ & $26.3 \pm 2.9$ & $3.3 \pm 1.7$ & $<2.9$ & $57.4 \pm 15.4$ & -1.03 \\
\hline & 022905.78 & 1.18 & -050740.81 & 1.92 & $C$ & & $6.5 \pm 1.4$ & $<8.1$ & $<6.2$ & $175.0 \pm 28.8$ & \\
\hline J0229.1-0223* & 022908.73 & 1.06 & -022311.17 & 1.75 & $S$ & $98.0 \pm 9.9$ & $98.0 \pm 9.9$ & $2.6 \pm 0.6$ & $<1.6$ & $25.7 \pm 11.4$ & -0.83 \\
\hline \multirow[t]{2}{*}{ J0229.2-0406 } & 022910.80 & 1.09 & -040609.58 & 1.77 & $M$ & $16.9 \pm 1.8$ & $9.0 \pm 1.3$ & $<4.8$ & $<3.6$ & $139.8 \pm 13.4$ & -0.92 \\
\hline & 022910.80 & 1.18 & -040605.26 & 1.79 & $C$ & & $7.9 \pm 1.3$ & $<6.5$ & $<4.7$ & $120.6 \pm 14.6$ & \\
\hline J0229.2-0243 & 022911.05 & 1.18 & -024343.88 & 1.85 & $S$ & $7.1 \pm 1.4$ & $7.1 \pm 1.4$ & $<6.8$ & $<6.2$ & $-1.0 \pm 72.2$ & $<-0.59$ \\
\hline J0229.2-0329 & 022911.45 & 1.37 & -032927.11 & 2.18 & $S$ & $14.3 \pm 3.2$ & $14.3 \pm 3.2$ & $11.9 \pm 8.3$ & $<6.5$ & $29.5 \pm 10.6$ & $<-1.07$ \\
\hline \multirow[t]{2}{*}{ J0229.2-0311* } & 022911.93 & 1.06 & -031138.03 & 1.75 & $M$ & $63.8 \pm 5.0$ & $41.5 \pm 4.3$ & $<2.1$ & $<1.9$ & $51.2 \pm 12.9$ & -0.68 \\
\hline & 022910.38 & 1.07 & -031122.13 & 1.76 & $C$ & & $22.2 \pm 2.5$ & $<3.3$ & $<2.9$ & $18.3 \pm 15.6$ & \\
\hline J0229.2-0511 & 022913.01 & 1.16 & -051119.46 & 1.79 & $S$ & $7.5 \pm 1.3$ & $7.5 \pm 1.3$ & $<6.2$ & $<4.7$ & $124.4 \pm 20.8$ & -0.45 \\
\hline J0229.2-0502 & 022914.38 & 1.08 & -050210.49 & 2.28 & $S$ & $11.5 \pm 2.2$ & $11.5 \pm 2.2$ & $<11.9$ & $<3.5$ & $2.3 \pm 3.0$ & $<-0.92$ \\
\hline J0229.2-0308 & 022914.87 & 1.25 & -030810.28 & 1.80 & $S$ & $7.1 \pm 1.3$ & $7.1 \pm 1.3$ & $<7.3$ & $<5.3$ & $102.2 \pm 18.7$ & 0.09 \\
\hline \multirow[t]{7}{*}{ J0229.3-0442* } & 022915.70 & 1.06 & -044216.30 & 1.75 & $M$ & $1058.0 \pm 71.1$ & $623.2 \pm 62.4$ & $3.6 \pm 0.1$ & $2.3 \pm 0.2$ & $145.7 \pm 2.0$ & -0.73 \\
\hline & 022916.10 & 1.06 & -044211.26 & 1.75 & $C$ & & $334.4 \pm 33.6$ & $6.8 \pm 0.3$ & $3.2 \pm 0.3$ & $59.3 \pm 1.4$ & \\
\hline & 022915.37 & 1.06 & -044214.06 & 1.75 & $C$ & & $26.9 \pm 2.9$ & $<2.9$ & $<1.6$ & $17.6 \pm 2.4$ & \\
\hline & $\begin{array}{lll}02 & 29 & 15.28\end{array}$ & 1.06 & -044211.05 & 1.75 & $C$ & & $26.9 \pm 2.9$ & $<2.9$ & $<1.6$ & $26.7 \pm 2.4$ & \\
\hline & 022915.87 & 1.09 & -044205.15 & 1.76 & $C$ & & $19.6 \pm 2.5$ & $<4.6$ & $<2.8$ & $130.4 \pm 6.2$ & \\
\hline & 022916.44 & 1.07 & -044209.23 & 1.79 & $C$ & & $15.2 \pm 2.1$ & $<5.2$ & $<2.6$ & $173.5 \pm 4.5$ & \\
\hline & 022916.70 & 1.15 & -044208.45 & 1.95 & $C$ & & $11.9 \pm 2.2$ & $<8.9$ & $<4.1$ & $155.6 \pm 6.0$ & \\
\hline J0229.3-0231 & 022916.34 & 1.10 & -023125.14 & 1.77 & $S$ & $8.6 \pm 1.3$ & $8.6 \pm 1.3$ & $<4.7$ & $<4.1$ & $49.7 \pm 42.7$ & -0.73 \\
\hline J0229.3-0403 & 022917.22 & 1.07 & -040327.83 & 1.76 & $S$ & $24.3 \pm 2.7$ & $24.3 \pm 2.7$ & $4.0 \pm 1.6$ & $<3.0$ & $90.8 \pm 14.3$ & -0.62 \\
\hline J0229.3-0313 & 022917.38 & 1.08 & -031329.49 & 1.77 & $S$ & $13.1 \pm 1.7$ & $13.1 \pm 1.7$ & $<4.3$ & $<3.6$ & $32.2 \pm 19.5$ & -0.50 \\
\hline J0229.3-0505 & 022918.64 & 1.21 & -050512.19 & 1.79 & $S$ & $7.4 \pm 1.3$ & $7.4 \pm 1.3$ & $<6.6$ & $<5.3$ & $96.2 \pm 24.2$ & -0.43 \\
\hline J0229.3-0427 & 022919.08 & 1.11 & -042756.53 & 2.16 & $S$ & $14.6 \pm 2.6$ & $14.6 \pm 2.6$ & $10.5 \pm 7.8$ & $<3.5$ & $11.6 \pm 3.0$ & $<-1.08$ \\
\hline J0229.3-0431 & 022919.97 & 1.10 & -043153.34 & 1.98 & $S$ & $6.4 \pm 1.3$ & $6.4 \pm 1.3$ & $<8.8$ & $<4.4$ & $172.0 \pm 8.6$ & $<-0.52$ \\
\hline J0229.4-0315 & 022921.58 & 1.07 & -031513.61 & 1.76 & $S$ & $12.7 \pm 1.5$ & $12.7 \pm 1.5$ & $<3.7$ & $<3.0$ & $43.0 \pm 11.3$ & -0.20 \\
\hline \multirow[t]{2}{*}{ J0229.4-0454 } & 022921.97 & 1.10 & -045424.03 & 1.82 & $M$ & $35.0 \pm 3.4$ & $20.7 \pm 2.8$ & $7.6 \pm 3.5$ & $<3.3$ & $27.1 \pm 4.3$ & -0.87 \\
\hline & 022922.04 & 1.07 & -045428.54 & 1.77 & $C$ & & $14.3 \pm 1.8$ & $<4.2$ & $<2.9$ & $12.3 \pm 8.4$ & \\
\hline J0229.4-0407 & 022922.34 & 1.07 & -040728.84 & 1.76 & $S$ & $16.2 \pm 1.9$ & $16.2 \pm 1.9$ & $<3.2$ & $\ldots$ & $\ldots$ & -1.02 \\
\hline J0229.4-0240 & 022922.92 & 1.14 & -024025.90 & 1.84 & $S$ & $6.1 \pm 1.2$ & $6.1 \pm 1.2$ & $<6.6$ & $<5.5$ & $175.0 \pm 38.6$ & -0.66 \\
\hline J0229.4-0350 & 022925.00 & 1.15 & -035037.58 & 1.82 & $S$ & $6.5 \pm 1.3$ & $6.5 \pm 1.3$ & $<6.2$ & $\ldots$ & $\ldots$ & $<-0.53$ \\
\hline J0229.4-0235 & 022925.11 & 1.07 & -023523.28 & 1.75 & $S$ & $28.6 \pm 3.0$ & $28.6 \pm 3.0$ & $<2.8$ & $<2.2$ & $58.7 \pm 5.0$ & -0.89 \\
\hline J0229.5-0303 & 022927.21 & 1.24 & -030324.05 & 1.82 & $S$ & $4.5 \pm 1.0$ & $4.5 \pm 1.0$ & $<7.6$ & $<5.1$ & $121.8 \pm 17.7$ & -0.32 \\
\hline J0229.5-0248 & 022931.80 & 1.06 & -024846.48 & 1.75 & $S$ & $27.5 \pm 2.9$ & $27.5 \pm 2.9$ & $<2.4$ & $<2.2$ & $46.7 \pm 11.5$ & -1.00 \\
\hline J0229.6-0433 & 022934.78 & 1.19 & -043312.50 & 1.81 & $S$ & $6.5 \pm 1.1$ & $6.5 \pm 1.1$ & $<7.2$ & $<4.4$ & $50.7 \pm 10.4$ & 0.45 \\
\hline J0229.6-0241 & 022934.90 & 1.10 & -024117.92 & 1.97 & $S$ & $5.8 \pm 1.1$ & $5.8 \pm 1.1$ & $<8.8$ & $<3.6$ & $12.8 \pm 5.2$ & $<-0.45$ \\
\hline J0229.6-0440 & 022935.62 & 1.26 & -044008.89 & 1.81 & $S$ & $5.3 \pm 1.1$ & $5.3 \pm 1.1$ & $<7.3$ & $<5.7$ & $94.0 \pm 31.6$ & $<-0.39$ \\
\hline J0229.6-0336 & 022936.50 & 1.78 & -033620.55 & 1.78 & $S$ & $23.7 \pm 3.9$ & $23.7 \pm 3.9$ & $18.3 \pm 7.3$ & $<4.6$ & $83.9 \pm 3.4$ & -0.99 \\
\hline J0229.6-0435 & 022936.54 & 1.12 & -043515.61 & 1.78 & $S$ & $9.1 \pm 1.3$ & $9.1 \pm 1.3$ & $<5.4$ & $<4.6$ & $49.7 \pm 26.2$ & -0.88 \\
\hline J0229.6-0509 & 022937.82 & 1.09 & -050924.68 & 1.76 & $S$ & $10.2 \pm 1.4$ & $10.2 \pm 1.4$ & $<4.3$ & $<3.9$ & $83.6 \pm 41.2$ & 0.07 \\
\hline J0229.6-0442 & 022937.97 & 1.08 & -044240.62 & 1.77 & $S$ & $11.4 \pm 1.5$ & $11.4 \pm 1.5$ & $<4.4$ & $<3.5$ & $32.2 \pm 14.4$ & -0.53 \\
\hline J0229.7-0330 & 022938.93 & 1.14 & -033020.23 & 1.83 & $S$ & $7.2 \pm 1.4$ & $7.2 \pm 1.4$ & $<6.3$ & $<5.4$ & $159.9 \pm 45.6$ & -0.54 \\
\hline J0229.7-0238* & 022939.06 & 1.06 & -023836.70 & 1.75 & $M$ & $121.9 \pm 11.7$ & $115.5 \pm 11.6$ & $3.2 \pm 0.4$ & $1.9 \pm 0.6$ & $70.9 \pm 7.5$ & -0.53 \\
\hline & 022938.54 & 1.18 & -023834.31 & 1.89 & $C$ & & $6.4 \pm 1.2$ & $<8.3$ & $<4.0$ & $33.9 \pm 7.1$ & \\
\hline J0229.7-0531 & 022939.85 & 1.08 & -053152.55 & 2.03 & $S$ & $5.7 \pm 1.2$ & $5.7 \pm 1.2$ & $<9.4$ & $<3.9$ & $1.8 \pm 5.9$ & -0.34 \\
\hline J0229.7-0247 & 022940.02 & 1.19 & -024704.64 & 1.84 & $S$ & $5.8 \pm 1.2$ & $5.8 \pm 1.2$ & $<7.0$ & $<5.9$ & $42.2 \pm 47.8$ & $<-0.45$ \\
\hline J0229.7-0341 & 022944.71 & 1.31 & -034108.17 & 1.84 & $S$ & $5.7 \pm 1.3$ & $5.7 \pm 1.3$ & $<7.9$ & $<6.4$ & $107.7 \pm 39.5$ & $<-0.44$ \\
\hline J0229.7-0524 & 022944.84 & 1.07 & -052430.99 & 1.75 & $S$ & $26.2 \pm 2.8$ & $26.2 \pm 2.8$ & $<2.7$ & $<2.6$ & $16.3 \pm 42.8$ & -0.55 \\
\hline J0229.8-0337 & 022947.59 & 1.08 & -033752.79 & 1.86 & $S$ & $5.7 \pm 1.0$ & $5.7 \pm 1.0$ & $<7.1$ & $<3.7$ & $4.8 \pm 7.8$ & -0.31 \\
\hline J0229.9-0330 & 022951.05 & 1.12 & -033050.18 & 1.88 & $S$ & $6.3 \pm 1.2$ & $6.3 \pm 1.2$ & $<7.4$ & $<5.1$ & $5.8 \pm 17.1$ & $<-0.50$ \\
\hline J0229.9-0447 & 022951.22 & 1.15 & -044740.02 & 1.80 & $S$ & $20.3 \pm 2.7$ & $20.3 \pm 2.7$ & $10.3 \pm 3.3$ & $<3.8$ & $45.2 \pm 4.8$ & -0.52 \\
\hline J0229.9-0225 & 022951.55 & 1.17 & -022559.28 & 2.63 & $S$ & $24.2 \pm 3.7$ & $24.2 \pm 3.7$ & $22.5 \pm 10.0$ & $<3.0$ & $166.3 \pm 1.1$ & $<-1.43$ \\
\hline J0229.9-0413 & 022952.59 & 1.42 & -041305.55 & 1.91 & $S$ & $5.2 \pm 1.2$ & $5.2 \pm 1.2$ & $<9.5$ & $<6.8$ & $58.3 \pm 21.8$ & -0.35 \\
\hline J0229.9-0228 & 022954.73 & 1.11 & -022841.95 & 1.76 & $M$ & $18.1 \pm 1.8$ & $10.5 \pm 1.4$ & $<4.9$ & $<2.7$ & $71.9 \pm 5.1$ & -1.00 \\
\hline & 022954.77 & 1.09 & -022844.62 & 1.75 & $C$ & & $7.5 \pm 1.0$ & $<4.5$ & $<2.5$ & $71.0 \pm 6.1$ & \\
\hline J0230.0-0249 & 022958.09 & 1.17 & -024931.02 & 1.76 & $M$ & $14.1 \pm 1.6$ & $7.5 \pm 1.2$ & $<6.1$ & $<3.5$ & $83.3 \pm 7.4$ & -0.71 \\
\hline & 022958.06 & 1.11 & -024927.93 & 1.76 & $C$ & & $6.6 \pm 1.0$ & $<5.0$ & $<3.4$ & $73.3 \pm 12.8$ & \\
\hline $\mathrm{J} 0230.0-0500$ & 022959.88 & 1.09 & -050101.41 & 1.76 & $S$ & $8.2 \pm 1.2$ & $8.2 \pm 1.2$ & $<4.2$ & $<3.9$ & $67.9 \pm 53.1$ & $<-0.69$ \\
\hline J0230.0-0331* & 023000.92 & 1.06 & -033149.65 & 1.75 & $M$ & $82.4 \pm 5.8$ & $42.7 \pm 4.5$ & $3.3 \pm 1.1$ & $<2.2$ & $61.8 \pm 6.0$ & -0.77 \\
\hline & 023001.41 & 1.06 & -033150.93 & 1.75 & $C$ & & $33.9 \pm 3.6$ & $<2.6$ & $<2.3$ & $33.6 \pm 10.6$ & \\
\hline & 023001.80 & 1.15 & -033146.97 & 1.90 & $C$ & & $5.8 \pm 1.1$ & $<8.3$ & $<3.9$ & $28.7 \pm 7.1$ & \\
\hline J0230.0-0440 & 023001.12 & 1.20 & -044059.61 & 1.82 & $S$ & $4.9 \pm 1.0$ & $4.9 \pm 1.0$ & $<7.0$ & $<5.4$ & $51.5 \pm 30.3$ & $<-0.33$ \\
\hline J0230.0-0324 & 023001.20 & 1.52 & -032431.20 & 1.93 & $S$ & $12.3 \pm 2.7$ & $12.3 \pm 2.7$ & $9.7 \pm 7.5$ & $<6.8$ & $59.2 \pm 15.8$ & -1.06 \\
\hline J0230.0-0402 & 023002.22 & 1.11 & -040259.72 & 1.78 & $S$ & $11.6 \pm 1.6$ & $11.6 \pm 1.6$ & $<5.7$ & $<3.5$ & $45.3 \pm 6.6$ & -0.50 \\
\hline J0230.0-0359 & 023002.75 & 1.40 & -035932.51 & 1.83 & $S$ & $5.5 \pm 1.1$ & $5.5 \pm 1.1$ & $<9.3$ & $<4.8$ & $116.6 \pm 8.5$ & $<-0.42$ \\
\hline J0230.1-0512 & 023004.37 & 1.07 & -051208.32 & 1.76 & $M$ & $24.7 \pm 2.3$ & $17.5 \pm 2.0$ & $<3.4$ & $<2.7$ & $133.4 \pm 9.6$ & -0.89 \\
\hline & 023004.53 & 1.14 & -051206.44 & 1.83 & $C$ & & $7.2 \pm 1.1$ & $<7.2$ & $<3.1$ & $38.3 \pm 4.0$ & \\
\hline J0230.1-0305 & 023005.78 & 1.10 & -030528.18 & 1.77 & $S$ & $11.1 \pm 1.5$ & $11.1 \pm 1.5$ & $<4.7$ & $<4.0$ & $53.3 \pm 23.2$ & 0.32 \\
\hline
\end{tabular}


C. Tasse et al.: The low-frequency counterpart of the XMM large scale structure survey, Online Material p 22

Table A.1. continued.

\begin{tabular}{|c|c|c|c|c|c|c|c|c|c|c|c|}
\hline Name & $\begin{array}{c}\text { RA } \\
\text { J2000 }\end{array}$ & $\begin{array}{c}\sigma_{\alpha} \\
\operatorname{arcsec}\end{array}$ & $\begin{array}{c}\text { Dec } \\
\text { J2000 }\end{array}$ & $\begin{array}{c}\sigma_{\delta} \\
\operatorname{arcsec}\end{array}$ & Type & $\begin{array}{l}S_{\text {int }} \\
\text { mJy }\end{array}$ & $\begin{array}{c}S_{\text {comp }} \\
\text { mJy }\end{array}$ & $\begin{array}{c}\text { Major axis } \\
\text { arcsec }\end{array}$ & $\begin{array}{c}\text { Minor axis } \\
\text { arcsec }\end{array}$ & $\begin{array}{c}\text { Position } \\
\text { Angle }\end{array}$ & $\begin{array}{c}\text { Spectral } \\
\text { index } \alpha_{325}^{1400}\end{array}$ \\
\hline \multirow[t]{2}{*}{ J0230.1-0341 } & 023005.89 & 1.07 & -034152.24 & 1.75 & $M$ & $39.7 \pm 3.7$ & $23.5 \pm 2.6$ & $<2.9$ & $<2.6$ & $33.1 \pm 18.0$ & -1.23 \\
\hline & 023005.87 & 1.34 & -034148.52 & 1.77 & $C$ & & $16.3 \pm 2.6$ & $9.4 \pm 4.9$ & $<4.3$ & $93.2 \pm 5.9$ & \\
\hline J0230.1-0457 & 023007.11 & 1.20 & -045715.15 & 1.80 & $S$ & $25.2 \pm 3.6$ & $25.2 \pm 3.6$ & $10.0 \pm 3.3$ & $6.6 \pm 2.7$ & $108.5 \pm 16.2$ & -0.28 \\
\hline J0230.2-0457 & 023011.56 & 1.09 & -045740.50 & 1.83 & $S$ & $13.1 \pm 1.9$ & $13.1 \pm 1.9$ & $6.4 \pm 3.8$ & $<3.8$ & $12.4 \pm 6.9$ & -0.64 \\
\hline J0230.2-0320 & 023013.43 & 1.23 & -032009.40 & 1.95 & $S$ & $6.3 \pm 1.2$ & $6.3 \pm 1.2$ & $<9.5$ & $<3.8$ & $34.8 \pm 4.9$ & $<-0.51$ \\
\hline J0230.3-0512 & 023015.16 & 1.08 & -051238.71 & 1.98 & $S$ & $4.9 \pm 1.0$ & $4.9 \pm 1.0$ & $<8.8$ & $<3.6$ & $2.0 \pm 5.5$ & $<-0.34$ \\
\hline J0230.3-0405 & 023016.48 & 1.18 & -040516.25 & 1.85 & $S$ & $5.3 \pm 1.1$ & $5.3 \pm 1.1$ & $<7.4$ & $<5.0$ & $141.0 \pm 18.6$ & $<-0.38$ \\
\hline J0230.3-0257 & 023017.85 & 1.18 & -025747.02 & 2.10 & $S$ & $13.5 \pm 2.7$ & $13.5 \pm 2.7$ & $<10.6$ & $<4.6$ & $20.0 \pm 6.0$ & $<-1.03$ \\
\hline \multirow[t]{2}{*}{$\mathrm{J} 0230.3-0432$} & 023018.91 & 1.11 & -043247.71 & 1.79 & $M$ & $22.5 \pm 2.2$ & $13.0 \pm 1.8$ & $5.3 \pm 3.7$ & $<3.3$ & $41.0 \pm 5.3$ & -0.94 \\
\hline & 023018.72 & 1.09 & -043247.68 & 1.76 & $C$ & & $9.5 \pm 1.4$ & $<4.1$ & $<3.5$ & $106.9 \pm 27.9$ & \\
\hline \multirow[t]{2}{*}{ J0230.4-0255* } & 023021.98 & 1.06 & -025503.86 & 1.75 & $M$ & $430.5 \pm 41.9$ & $417.7 \pm 41.9$ & $10.0 \pm 0.2$ & $2.7 \pm 0.2$ & $156.8 \pm 0.4$ & -1.07 \\
\hline & 023022.44 & 1.10 & -025500.67 & 1.80 & $C$ & & $12.7 \pm 1.9$ & $<6.1$ & $<3.5$ & $149.4 \pm 6.5$ & \\
\hline \multirow[t]{3}{*}{ J0230.4-0412* } & 023023.42 & 1.06 & -041226.54 & 1.75 & $M$ & $493.4 \pm 43.3$ & $429.0 \pm 43.0$ & $4.0 \pm 0.2$ & $2.5 \pm 0.3$ & $46.2 \pm 2.8$ & -0.55 \\
\hline & 023023.83 & 1.06 & -041221.76 & 1.75 & $C$ & & $37.3 \pm 4.1$ & $<2.7$ & $<2.3$ & $177.7 \pm 10.8$ & \\
\hline & 023023.09 & 1.06 & -041223.30 & 1.75 & $C$ & & $27.2 \pm 2.9$ & $<2.1$ & $<1.6$ & $21.2 \pm 6.1$ & \\
\hline J0230.4-0305 & 023023.93 & 1.07 & -030525.57 & 2.45 & $S$ & $24.5 \pm 4.0$ & $24.5 \pm 4.0$ & $17.3 \pm 8.8$ & $<3.3$ & $177.8 \pm 1.7$ & $<-1.44$ \\
\hline J0230.4-0432 & 023024.17 & 1.07 & -043213.57 & 1.75 & $S$ & $26.7 \pm 2.9$ & $26.7 \pm 2.9$ & $<3.0$ & $<2.7$ & $43.5 \pm 18.9$ & -0.84 \\
\hline J0230.5-0442 & 023027.32 & 1.30 & -044242.05 & 1.83 & $S$ & $6.6 \pm 1.3$ & $6.6 \pm 1.3$ & $<7.8$ & $<6.3$ & $97.5 \pm 32.3$ & -0.44 \\
\hline \multirow[t]{2}{*}{ J0230.5-0410 } & 023027.66 & 1.36 & -041036.53 & 2.08 & $M$ & $13.9 \pm 1.6$ & $7.1 \pm 1.1$ & $<11.5$ & $<2.1$ & $142.8 \pm 1.1$ & -1.08 \\
\hline & 023027.51 & 1.38 & -041034.40 & 2.09 & $C$ & & $6.8 \pm 1.1$ & $<11.7$ & $<2.1$ & $37.4 \pm 1.2$ & \\
\hline J0230.5-0346 & 023032.78 & 1.08 & -034643.58 & 1.94 & $S$ & $5.0 \pm 1.0$ & $5.0 \pm 1.0$ & $<8.3$ & $<3.7$ & $172.9 \pm 6.6$ & -0.49 \\
\hline J0230.6-0309* & 023033.39 & 1.06 & -030906.78 & 1.75 & $S$ & $115.0 \pm 11.6$ & $115.0 \pm 11.6$ & $2.5 \pm 0.5$ & $<1.5$ & $80.2 \pm 10.2$ & -0.55 \\
\hline \multirow[t]{2}{*}{ J0230.6-0421* } & 023033.50 & 1.06 & -042107.66 & 1.75 & $M$ & $175.0 \pm 12.7$ & $92.6 \pm 9.5$ & $4.7 \pm 0.7$ & $<1.6$ & $142.3 \pm 1.8$ & -0.59 \\
\hline & 023033.71 & 1.06 & -042107.13 & 1.75 & $C$ & & $82.4 \pm 8.5$ & $5.4 \pm 0.9$ & $<1.7$ & $35.4 \pm 1.6$ & \\
\hline J0230.6-0328 & 023035.86 & 1.14 & -032802.94 & 1.87 & $S$ & $7.4 \pm 1.4$ & $7.4 \pm 1.4$ & $<7.7$ & $<4.2$ & $28.8 \pm 8.6$ & -0.04 \\
\hline \multirow[t]{4}{*}{ J0230.7-0328* } & 023040.92 & 1.06 & -032837.58 & 1.75 & $M$ & $228.5 \pm 15.3$ & $102.4 \pm 10.3$ & $<1.6$ & $<1.5$ & $148.8 \pm 6.0$ & -0.90 \\
\hline & 023040.63 & 1.28 & -032833.64 & 1.75 & $C$ & & $101.6 \pm 10.9$ & $26.1 \pm 3.5$ & $<1.9$ & $95.6 \pm 0.4$ & \\
\hline & 023041.14 & 1.08 & -032848.53 & 1.77 & $C$ & & $15.1 \pm 2.0$ & $<4.7$ & $<3.3$ & $145.3 \pm 8.4$ & \\
\hline & 023041.31 & 1.09 & -032845.53 & 1.88 & $C$ & & $9.4 \pm 1.6$ & $<7.4$ & $<3.9$ & $11.1 \pm 7.1$ & \\
\hline \multirow[t]{2}{*}{ J0230.7-0256* } & 023043.37 & 1.49 & -025610.68 & 2.24 & $M$ & $31.4 \pm 4.9$ & $16.1 \pm 3.6$ & $14.2 \pm 8.9$ & $<6.6$ & $34.4 \pm 8.9$ & 0.56 \\
\hline & 023042.98 & 1.38 & -025600.30 & 2.11 & $C$ & & $15.4 \pm 3.3$ & $12.2 \pm 7.3$ & $<7.2$ & $30.8 \pm 14.6$ & \\
\hline J0230.8-0424 & 023048.35 & 1.09 & -042420.71 & 2.08 & $S$ & $5.1 \pm 1.0$ & $5.1 \pm 1.0$ & $<10.0$ & $<4.0$ & $4.7 \pm 5.3$ & $<-0.37$ \\
\hline J0230.9-0459 & 023054.17 & 1.24 & -045919.10 & 2.08 & $S$ & $4.8 \pm 1.0$ & $4.8 \pm 1.0$ & $<10.6$ & $<5.2$ & $26.5 \pm 8.4$ & $<-0.32$ \\
\hline \multirow[t]{2}{*}{ J0231.0-0451* } & 023057.54 & 1.06 & -045136.75 & 1.75 & $M$ & $103.4 \pm 10.0$ & $97.0 \pm 9.9$ & $8.8 \pm 0.7$ & $4.5 \pm 0.6$ & $36.8 \pm 2.4$ & -0.56 \\
\hline & 023057.04 & 1.10 & -045132.92 & 1.86 & $C$ & & $6.5 \pm 1.1$ & $<7.2$ & $<4.2$ & $15.5 \pm 9.0$ & \\
\hline J0231.0-0447 & 023057.75 & 1.20 & -044754.34 & 1.95 & $S$ & $5.4 \pm 1.1$ & $5.4 \pm 1.1$ & $<9.1$ & $<4.8$ & $28.8 \pm 9.1$ & $<-0.41$ \\
\hline
\end{tabular}

An asterisk $(*)$ after the sourcename indicates this source was also detected at $74 \mathrm{MHz}$.

Description of columns:

1. Source Name.

2. Right Ascension (J2000)

3. Right Ascension uncertainty in arcseconds.

4. Declination (J2000)

5. Declination uncertainty in arcseconds.

6. Source type: Single (S), Multiple (M) or Component (C) of multiple source.

7. Integrated flux density in mJy. An asterisk $(*)$ indicates the flux density has been calculated using pixel based method.

8. Flux density of individual componant in mJy as derived through the Gaussian fitting method.

9. Size of Major Axis in arcseconds, or the $2 \sigma$ upper limit if the source is unresolved.

10. Size of Minor Axis in arcseconds, or the $2 \sigma$ upper limit if the source is unresolved.

11. PA of Major axis in degrees.

12. Spectral index with respect to NVSS flux density $(1.4 \mathrm{GHz})$ 
Table A.2. The $74 \mathrm{MHz}$ source list.

\begin{tabular}{|c|c|c|c|c|c|c|c|c|c|c|}
\hline Name & $\begin{array}{c}\text { RA } \\
\text { J2000 }\end{array}$ & $\begin{array}{c}\sigma_{\alpha} \\
\operatorname{arcsec}\end{array}$ & $\begin{array}{c}\text { Dec } \\
\text { J2000 }\end{array}$ & $\begin{array}{c}\sigma_{\delta} \\
\operatorname{arcsec}\end{array}$ & Type & $\begin{array}{c}S_{\text {comp }} \\
\text { Jy }\end{array}$ & $\begin{array}{c}\text { Major axis } \\
\text { arcsec }\end{array}$ & $\begin{array}{c}\text { Minor axis } \\
\text { arcsec }\end{array}$ & $\begin{array}{c}\text { Position } \\
\text { Angle }\end{array}$ & $\begin{array}{c}\text { Spectral } \\
\text { index } \alpha_{74}^{1400}\end{array}$ \\
\hline J0157.7-0500 & 015743.28 & 3.7 & -050006.50 & 2.5 & $\bar{S}$ & $1.69 \pm 0.15$ & $40.0 \pm 13.3$ & $<33.2$ & $35.2 \pm 9.8$ & -0.97 \\
\hline J0158.1-0311 & 015807.51 & 4.3 & -031153.89 & 3.5 & $S$ & $0.48 \pm 0.09$ & $<46.9$ & $<31.1$ & $32.3 \pm 28.1$ & -0.47 \\
\hline J0159.2-0400 & 015912.69 & 4.2 & -040007.77 & 4.4 & $S$ & $0.65 \pm 0.11$ & $42.1 \pm 28.6$ & $<33.2$ & $13.5 \pm 11.9$ & -0.93 \\
\hline J0159.2-0605 & 015913.15 & 5.7 & -060530.76 & 4.3 & $S$ & $0.63 \pm 0.14$ & $<66.4$ & $<51.7$ & $92.7 \pm 43.1$ & -0.71 \\
\hline J0159.4-0408 & 015925.02 & 4.1 & -040844.72 & 3.4 & $S$ & $0.68 \pm 0.10$ & $<55.5$ & $<35.5$ & $27.5 \pm 18.3$ & -0.67 \\
\hline \multirow[t]{2}{*}{ J0159.7-0408 } & 015944.11 & 4.3 & -040808.00 & 4.8 & $M$ & $0.33 \pm 0.08$ & $<62.7$ & $<24.1$ & $163.1 \pm 14.3$ & -0.71 \\
\hline & 015945.32 & 4.4 & -040757.42 & 3.2 & $C$ & $0.32 \pm 0.06$ & $<41.0$ & $<24.4$ & $53.6 \pm 28.1$ & \\
\hline J0159.9-0150 & 015951.21 & 4.3 & -015004.25 & 4.2 & $S$ & $0.92 \pm 0.15$ & $41.8 \pm 26.3$ & $<45.4$ & $178.4 \pm 19.7$ & -0.89 \\
\hline J0200.4-0257 & 020021.95 & 4.5 & -025731.60 & 3.4 & $S$ & $0.30 \pm 0.07$ & $<37.2$ & $\ldots$ & $\ldots$ & -0.66 \\
\hline J0200.5-0346 & 020031.33 & 3.6 & -034623.36 & 2.4 & $S$ & $1.19 \pm 0.11$ & $31.0 \pm 14.4$ & $<30.3$ & $48.9 \pm 12.9$ & -0.85 \\
\hline J0201.0-0217 & 020059.97 & 6.7 & -021739.82 & 5.7 & $S$ & $0.77 \pm 0.18$ & $<82.2$ & $\ldots$ & $\ldots$ & -0.75 \\
\hline J0201.0-0324 & 020101.21 & 5.5 & -032448.56 & 3.0 & $S$ & $1.38 \pm 0.18$ & $77.9 \pm 23.9$ & $<45.1$ & $80.6 \pm 6.7$ & -0.82 \\
\hline J0201.3-0421 & 020120.83 & 4.6 & -042104.69 & 3.6 & $S$ & $0.42 \pm 0.08$ & $<58.3$ & $<26.5$ & $46.5 \pm 14.2$ & -0.75 \\
\hline \multirow[t]{2}{*}{ J0201.6-0024 } & 020136.89 & 5.2 & -002407.21 & 4.4 & $M$ & $0.32 \pm 0.09$ & $<49.5$ & $<36.4$ & $46.0 \pm 52.8$ & -0.43 \\
\hline & 020138.28 & 4.7 & -002355.14 & 4.4 & $C$ & $0.23 \pm 0.06$ & $<48.1$ & $<21.6$ & $149.3 \pm 25.4$ & \\
\hline J0201.7-0211 & 020143.17 & 4.2 & -021150.45 & 4.3 & $S$ & $1.20 \pm 0.16$ & $62.3 \pm 22.8$ & $<46.9$ & $13.9 \pm 10.3$ & -0.77 \\
\hline J0201.8-0355 & 020145.42 & 3.7 & -035511.66 & 2.7 & $S$ & $0.55 \pm 0.07$ & $<37.1$ & $<25.7$ & $173.7 \pm 24.7$ & -0.75 \\
\hline J0201.8-0419 & 020145.69 & 3.6 & -041926.52 & 2.4 & $S$ & $1.14 \pm 0.10$ & $25.9 \pm 15.1$ & $<34.7$ & $54.5 \pm 29.3$ & -0.71 \\
\hline J0201.8-0609 & 020146.10 & 4.9 & -060939.83 & 3.6 & $S$ & $0.98 \pm 0.15$ & $52.9 \pm 24.6$ & $<41.0$ & $54.3 \pm 11.4$ & -0.81 \\
\hline J0201.8-0211 & 020147.12 & 6.0 & -021200.37 & 3.9 & $S$ & $0.44 \pm 0.10$ & $<69.8$ & $<39.5$ & $102.0 \pm 20.7$ & -0.45 \\
\hline J0202.1-0558 & 020207.30 & 4.3 & -055859.54 & 3.5 & $S$ & $0.50 \pm 0.09$ & $<53.3$ & $<29.2$ & $39.0 \pm 18.0$ & -0.28 \\
\hline J0202.1-0728 & 020207.46 & 6.2 & -072840.06 & 4.1 & $S$ & $1.29 \pm 0.22$ & $75.4 \pm 31.1$ & $<46.4$ & $62.9 \pm 8.6$ & -1.08 \\
\hline J0202.6-0310 & 020236.86 & 6.0 & -031007.64 & 5.1 & $S$ & $0.57 \pm 0.13$ & $<78.6$ & $<58.4$ & $49.7 \pm 34.9$ & -0.63 \\
\hline J0202.9-0048 & 020251.81 & 3.6 & -004858.67 & 2.3 & $S$ & $1.83 \pm 0.15$ & $28.8 \pm 12.3$ & $<35.1$ & $122.2 \pm 25.7$ & -0.52 \\
\hline J0202.9-0412 & 020255.86 & 4.1 & -041258.94 & 2.8 & $S$ & $0.86 \pm 0.10$ & $36.1 \pm 18.6$ & $<38.8$ & $63.5 \pm 18.3$ & -0.68 \\
\hline J0202.9-0605 & 020256.61 & 3.4 & -060523.97 & 2.1 & $S$ & $3.09 \pm 0.19$ & $35.5 \pm 7.5$ & $18.4 \pm 11.3$ & $41.5 \pm 6.4$ & -0.76 \\
\hline $\mathrm{J} 0203.3+0006$ & $\begin{array}{lll}02 & 03 & 19.02\end{array}$ & 4.5 & +000633.42 & 3.8 & $S$ & $0.53 \pm 0.11$ & $<44.1$ & $\ldots$ & $\ldots$ & -0.49 \\
\hline J0203.4-0257 & 020326.39 & 4.6 & -025708.40 & 4.5 & $S$ & $0.21 \pm 0.06$ & $<47.5$ & $<30.3$ & $3.4 \pm 38.4$ & -0.56 \\
\hline J0203.5-0354 & 020329.76 & 5.9 & -035459.01 & 5.3 & $S$ & $0.41 \pm 0.10$ & $<71.9$ & $<52.0$ & $136.6 \pm 37.3$ & -0.82 \\
\hline J0203.7-0058 & 020341.53 & 4.8 & -005822.38 & 4.0 & $S$ & $0.25 \pm 0.06$ & $<40.8$ & $\ldots$ & $\ldots$ & -0.61 \\
\hline J0203.8-0654 & 020346.53 & 5.1 & -065450.73 & 5.7 & $S$ & $0.37 \pm 0.10$ & $<68.5$ & $<41.8$ & $12.6 \pm 27.9$ & -0.76 \\
\hline J0203.8-0811 & 020346.79 & 3.5 & -081116.70 & 2.2 & $S$ & $2.63 \pm 0.19$ & $25.4 \pm 11.8$ & $20.0 \pm 14.0$ & $45.7 \pm 41.5$ & -0.63 \\
\hline $\mathrm{J} 0204.0+0025$ & 020402.08 & 5.5 & +002536.32 & 5.4 & $S$ & $0.58 \pm 0.14$ & $<80.4$ & $<39.1$ & $36.1 \pm 16.2$ & -0.63 \\
\hline J0204.1-0408 & 020405.66 & 4.2 & -040832.02 & 3.4 & $S$ & $0.32 \pm 0.06$ & $<38.9$ & $\ldots$ & $\ldots$ & -0.64 \\
\hline J0204.1-0528 & 020406.09 & 4.5 & -052840.92 & 4.0 & $S$ & $0.77 \pm 0.12$ & $35.1 \pm 27.5$ & $<52.7$ & $4.3 \pm 46.1$ & -0.70 \\
\hline J0204.5-0535 & 020431.11 & 4.4 & -053512.91 & 3.8 & $S$ & $0.37 \pm 0.07$ & $<51.0$ & $<31.1$ & $35.5 \pm 24.5$ & -0.53 \\
\hline J0204.6-0250 & 020433.67 & 4.7 & -025001.98 & 4.6 & $S$ & $0.25 \pm 0.06$ & $<53.1$ & $<37.4$ & $4.3 \pm 39.2$ & -0.58 \\
\hline J0204.6-0619 & 020436.40 & 3.7 & -061938.16 & 12.6 & $S$ & $6.49 \pm 0.55$ & $426.2 \pm 59.0$ & $30.7 \pm 12.9$ & $2.7 \pm 0.6$ & -1.44 \\
\hline J0204.6-0428 & 020437.10 & 4.7 & -042832.10 & 3.9 & $S$ & $0.79 \pm 0.11$ & $61.3 \pm 24.0$ & $<39.1$ & $43.1 \pm 8.5$ & -0.76 \\
\hline J0204.7-0725 & 020442.97 & 5.0 & -072539.29 & 5.4 & $S$ & $0.55 \pm 0.13$ & $<68.9$ & $<44.4$ & $8.6 \pm 27.6$ & -0.63 \\
\hline J0204.9-0655 & 020455.00 & 3.6 & -065508.33 & 2.5 & $S$ & $2.54 \pm 0.21$ & $46.0 \pm 11.3$ & $25.1 \pm 13.5$ & $17.0 \pm 9.1$ & -0.52 \\
\hline J0204.9-0715 & 020455.18 & 3.6 & -071555.51 & 2.4 & $S$ & $2.07 \pm 0.18$ & $34.6 \pm 12.6$ & $21.7 \pm 16.2$ & $141.8 \pm 19.3$ & -0.70 \\
\hline J0205.0-0429 & 020500.33 & 3.9 & -042946.91 & 2.7 & $S$ & $0.68 \pm 0.08$ & $30.9 \pm 19.7$ & $<29.8$ & $58.0 \pm 13.2$ & -0.83 \\
\hline J0205.4-0225 & 020521.27 & 3.7 & -022533.60 & 2.3 & $S$ & $0.95 \pm 0.09$ & $<39.4$ & $<29.0$ & $70.8 \pm 18.9$ & -0.57 \\
\hline J0205.4-0301 & 020523.30 & 3.9 & -030100.78 & 3.0 & $S$ & $0.54 \pm 0.08$ & $<40.9$ & $<34.2$ & $153.1 \pm 49.2$ & -0.84 \\
\hline J0205.4-0304 & 020523.70 & 4.7 & -030445.08 & 5.2 & $S$ & $0.32 \pm 0.08$ & $<64.3$ & $<37.8$ & $0.0 \pm 24.5$ & -0.59 \\
\hline J0205.4-0642 & 020524.20 & 4.3 & -064205.24 & 3.9 & $S$ & $0.51 \pm 0.10$ & $<53.0$ & $<36.6$ & $14.6 \pm 28.9$ & -1.05 \\
\hline J0205.7-0134 & 020539.81 & 4.6 & -013429.56 & 4.1 & $S$ & $0.32 \pm 0.07$ & $<47.6$ & $<38.3$ & $3.7 \pm 60.8$ & -0.70 \\
\hline J0205.7-0819 & 020541.45 & 4.1 & -081942.25 & 3.1 & $S$ & $1.06 \pm 0.15$ & $<50.2$ & $<43.0$ & $37.4 \pm 47.3$ & -0.72 \\
\hline J0206.1-0432 & 020603.54 & 3.9 & -043259.50 & 3.0 & $S$ & $0.48 \pm 0.07$ & $<40.6$ & $<30.9$ & $176.6 \pm 35.1$ & -0.66 \\
\hline \multirow[t]{2}{*}{ J0206.1-0503 } & 020603.86 & 3.6 & -050329.91 & 2.3 & $M$ & $1.46 \pm 0.12$ & $37.2 \pm 12.1$ & $<29.1$ & $136.8 \pm 8.3$ & -0.63 \\
\hline & 020605.42 & 3.6 & -050337.25 & 2.4 & $C$ & $1.26 \pm 0.11$ & $38.0 \pm 12.6$ & $<23.5$ & $28.3 \pm 6.5$ & \\
\hline J0206.1-0511 & 020604.15 & 3.8 & -051131.50 & 2.1 & $S$ & $6.20 \pm 0.36$ & $131.9 \pm 9.3$ & $24.3 \pm 8.5$ & $108.8 \pm 0.8$ & -0.94 \\
\hline J0206.2-0604 & 020611.46 & 3.9 & -060403.15 & 2.8 & $S$ & $0.52 \pm 0.07$ & $<41.9$ & $<23.3$ & $41.4 \pm 17.5$ & -0.46 \\
\hline J0206.3-0553 & 020617.02 & 3.7 & -055323.49 & 2.4 & $S$ & $1.41 \pm 0.13$ & $42.4 \pm 12.7$ & $<26.4$ & $49.5 \pm 6.4$ & -0.41 \\
\hline J0206.5-0344 & 020627.36 & 3.7 & -034421.41 & 4.1 & $S$ & $1.57 \pm 0.15$ & $104.6 \pm 19.6$ & $<31.3$ & $17.0 \pm 2.5$ & -0.64 \\
\hline J0207.1-0854 & 020704.10 & 3.7 & -085443.41 & 2.5 & $S$ & $1.95 \pm 0.19$ & $32.9 \pm 14.5$ & $<36.7$ & $29.6 \pm 19.4$ & -0.76 \\
\hline J0207.1-0149 & 020707.15 & 4.3 & -014955.54 & 3.6 & $S$ & $0.17 \pm 0.04$ & $<37.5$ & $<23.1$ & $32.8 \pm 37.9$ & -0.59 \\
\hline J0207.2-0704 & 020714.06 & 4.2 & -070416.63 & 3.8 & $S$ & $0.59 \pm 0.09$ & $35.2 \pm 26.8$ & $<36.6$ & $21.5 \pm 15.8$ & -0.84 \\
\hline J0207.3-0812 & 020718.74 & 5.4 & -081250.63 & 4.3 & $S$ & $0.62 \pm 0.13$ & $<68.0$ & $<44.7$ & $55.2 \pm 25.3$ & -0.88 \\
\hline J0207.6-0158 & 020732.93 & 4.9 & -015839.03 & 4.1 & $S$ & $0.27 \pm 0.06$ & $<56.9$ & $<31.5$ & $45.3 \pm 22.7$ & -0.77 \\
\hline J0207.6+0006 & 020735.39 & 3.9 & +000600.92 & 2.6 & $S$ & $1.04 \pm 0.12$ & $<45.2$ & $<33.9$ & $110.3 \pm 24.0$ & -1.00 \\
\hline J0207.8-0304 & 020750.19 & 5.2 & -030454.57 & 4.9 & $S$ & $0.30 \pm 0.07$ & $<64.6$ & $<43.9$ & $32.5 \pm 31.5$ & -0.96 \\
\hline J0207.9-0053 & 020751.83 & 4.2 & -005358.80 & 3.7 & $S$ & $0.77 \pm 0.12$ & $<58.3$ & $<43.4$ & $169.3 \pm 27.1$ & -0.80 \\
\hline J0207.9-0052 & 020752.15 & 4.5 & -005252.93 & 4.0 & $S$ & $0.42 \pm 0.09$ & $<53.0$ & $<34.4$ & $154.3 \pm 27.8$ & -0.64 \\
\hline J0207.9-0115 & 020754.04 & 3.6 & -011530.04 & 2.3 & $S$ & $0.76 \pm 0.08$ & $<32.2$ & $<24.0$ & $99.2 \pm 26.9$ & -0.83 \\
\hline J0208.0-0527 & 020757.53 & 3.4 & -052719.58 & 2.0 & $S$ & $2.50 \pm 0.14$ & $29.3 \pm 7.2$ & $<23.4$ & $46.6 \pm 5.1$ & -0.63 \\
\hline \multirow[t]{2}{*}{$\mathrm{J} 0208.2+0022$} & 020810.69 & 3.4 & +00 2301.98 & 2.1 & $M$ & $3.10 \pm 0.19$ & $23.0 \pm 9.9$ & $20.2 \pm 10.9$ & $106.8 \pm 51.5$ & -0.71 \\
\hline & 020809.84 & 4.5 & +002218.33 & 4.0 & $C$ & $0.24 \pm 0.06$ & $<44.8$ & $<17.7$ & $147.1 \pm 22.5$ & \\
\hline J0208.3-0708 & 020819.93 & 4.4 & -070816.68 & 3.7 & $S$ & $1.28 \pm 0.16$ & $65.3 \pm 21.3$ & $<45.7$ & $39.5 \pm 8.8$ & -0.78 \\
\hline J0208.4-0047 & 020825.99 & 3.4 & -004749.13 & 2.0 & $S$ & $2.45 \pm 0.15$ & $<26.9$ & $<24.7$ & $94.5 \pm 35.5$ & -0.49 \\
\hline J0208.5-0637 & 020829.10 & 4.6 & -063711.07 & 4.1 & $S$ & $0.26 \pm 0.06$ & $<51.3$ & $<30.0$ & $32.6 \pm 26.7$ & -0.62 \\
\hline J0208.5-0226 & 020832.44 & 3.9 & -022644.87 & 2.9 & $S$ & $0.52 \pm 0.07$ & $<49.5$ & $<23.8$ & $39.0 \pm 11.7$ & -0.81 \\
\hline J0208.8-0516 & 020848.18 & 4.8 & -051622.13 & 3.4 & $S$ & $0.25 \pm 0.05$ & $<57.6$ & $<23.0$ & $57.3 \pm 13.8$ & -0.69 \\
\hline
\end{tabular}


Table A.2. continued.

\begin{tabular}{|c|c|c|c|c|c|c|c|c|c|c|}
\hline Name & $\begin{array}{c}\text { RA } \\
\text { J2000 }\end{array}$ & $\begin{array}{c}\sigma_{\alpha} \\
\operatorname{arcsec}\end{array}$ & $\begin{array}{c}\text { Dec } \\
\text { J2000 }\end{array}$ & $\begin{array}{c}\sigma_{\delta} \\
\operatorname{arcsec}\end{array}$ & Type & $\underset{\substack{\text { comp } \\
\text { Jy }}}{S_{\text {na }}}$ & $\begin{array}{c}\text { Major axis } \\
\text { arcsec }\end{array}$ & $\begin{array}{c}\text { Minor axis } \\
\text { arcsec }\end{array}$ & $\begin{array}{c}\text { Position } \\
\text { Angle }\end{array}$ & $\begin{array}{c}\text { Spectral } \\
\text { index } \alpha_{74}^{1400}\end{array}$ \\
\hline \multirow[t]{2}{*}{ J0208.9+0109 } & 020853.88 & 4.5 & +010959.53 & 3.4 & $M$ & $0.65 \pm 0.12$ & $<50.4$ & $<30.7$ & $127.1 \pm 22.5$ & -0.78 \\
\hline & 020854.26 & 4.3 & +010943.18 & 3.9 & C & $0.45 \pm 0.10$ & $<53.0$ & $<20.6$ & $32.3 \pm 15.3$ & \\
\hline J0209.0-0307 & 020901.99 & 4.0 & -030720.39 & 2.8 & $S$ & $0.66 \pm 0.08$ & $25.6 \pm 23.2$ & $<38.7$ & $108.7 \pm 29.0$ & -0.66 \\
\hline J0209.1-0622 & 020906.85 & 5.3 & -062255.25 & 4.3 & $S$ & $0.37 \pm 0.08$ & $<69.3$ & $<33.8$ & $47.9 \pm 16.1$ & -0.78 \\
\hline J0209.1-0308 & 020907.12 & 3.5 & -030808.51 & 2.2 & $S$ & $1.27 \pm 0.09$ & $17.6 \pm 15.4$ & $<31.5$ & $18.5 \pm 84.8$ & -0.59 \\
\hline J0209.5-0807 & 020928.83 & 4.6 & -080747.21 & 4.7 & $S$ & $0.90 \pm 0.16$ & $48.7 \pm 28.9$ & $<44.5$ & $21.1 \pm 15.2$ & -1.02 \\
\hline J0209.5-0438 & 020930.97 & 4.3 & -043829.77 & 3.6 & $S$ & $0.49 \pm 0.08$ & $37.4 \pm 26.0$ & $<34.7$ & $36.6 \pm 13.9$ & -0.27 \\
\hline \multirow[t]{2}{*}{ J0209.5-0736 } & 020931.89 & 3.4 & -073644.66 & 2.1 & $M$ & $3.63 \pm 0.22$ & $34.1 \pm 7.9$ & $30.3 \pm 8.3$ & $175.0 \pm 27.4$ & -0.82 \\
\hline & 020934.92 & 5.0 & -073608.60 & 3.8 & $C$ & $0.50 \pm 0.11$ & $<53.2$ & $<43.8$ & $78.6 \pm 58.6$ & \\
\hline $\mathrm{J} 0209.6+0028$ & 020938.03 & 3.8 & +002836.81 & 3.2 & $S$ & $0.41 \pm 0.07$ & $<40.7$ & $<13.5$ & $12.8 \pm 14.3$ & -1.05 \\
\hline J0209.6-0059 & 020938.05 & 3.6 & -005957.52 & 2.4 & $S$ & $0.74 \pm 0.08$ & $<30.1$ & $<26.9$ & $103.2 \pm 69.6$ & -0.48 \\
\hline J0209.7-0339 & 020939.30 & 3.9 & -033933.21 & 3.4 & $S$ & $0.86 \pm 0.10$ & $53.7 \pm 18.6$ & $<30.8$ & $27.0 \pm 6.9$ & -0.84 \\
\hline $\mathrm{J} 0209.7+0020$ & 020939.96 & 3.7 & +002047.11 & 2.7 & $S$ & $0.64 \pm 0.08$ & $<35.1$ & $<22.3$ & $169.1 \pm 22.3$ & -0.63 \\
\hline J0209.7-0338 & 020941.48 & 4.3 & -033841.55 & 4.4 & $S$ & $0.40 \pm 0.08$ & $<62.3$ & $<35.1$ & $11.5 \pm 18.2$ & -0.72 \\
\hline J0209.8-0137 & 020945.05 & 3.4 & -013737.51 & 2.0 & $S$ & $4.57 \pm 0.24$ & $19.7 \pm 7.7$ & $<23.1$ & $77.7 \pm 9.6$ & -0.57 \\
\hline J0209.8-0512 & 020945.62 & 3.7 & -051211.58 & 2.6 & $S$ & $0.54 \pm 0.06$ & $27.7 \pm 18.4$ & $<16.3$ & $38.2 \pm 7.9$ & -0.58 \\
\hline J0209.8-0200 & 020945.90 & 4.9 & -020056.08 & 4.3 & $S$ & $0.29 \pm 0.07$ & $<52.5$ & $<35.1$ & $36.1 \pm 34.9$ & -0.66 \\
\hline \multirow[t]{2}{*}{ J0209.8-0237 } & 020946.21 & 3.9 & -023710.35 & 3.4 & $M$ & $0.26 \pm 0.04$ & $<46.5$ & $<12.3$ & $25.9 \pm 12.2$ & -0.52 \\
\hline & 020947.15 & 4.4 & -023709.39 & 3.3 & $C$ & $0.09 \pm 0.03$ & $<31.4$ & $<6.6$ & $53.5 \pm 30.0$ & \\
\hline J0209.9-0511 & 020952.26 & 4.8 & -051135.21 & 4.4 & $S$ & $0.17 \pm 0.04$ & $<53.5$ & $<25.6$ & $36.4 \pm 22.7$ & -0.69 \\
\hline J0210.2-0505 & 021012.34 & 4.0 & -050532.35 & 2.9 & $S$ & $0.29 \pm 0.05$ & $<35.1$ & $<24.8$ & $44.4 \pm 36.6$ & -0.30 \\
\hline J0210.3-0007 & 021016.09 & 4.7 & -000709.56 & 4.2 & $S$ & $0.27 \pm 0.06$ & $<52.9$ & $<25.7$ & $145.6 \pm 21.5$ & -0.55 \\
\hline \multirow[t]{2}{*}{ J0210.3-0344 } & 021016.19 & 3.5 & -034411.35 & 2.0 & $M$ & $3.99 \pm 0.23$ & $83.4 \pm 7.1$ & $24.9 \pm 8.4$ & $97.8 \pm 1.5$ & -0.83 \\
\hline & 021013.00 & 3.5 & -034407.77 & 2.1 & $C$ & $1.00 \pm 0.07$ & $<30.1$ & $<23.9$ & $51.2 \pm 21.7$ & \\
\hline J0210.3-0417 & 021017.26 & 5.1 & -041702.92 & 3.7 & $S$ & $0.61 \pm 0.09$ & $62.7 \pm 25.8$ & $<30.8$ & $53.7 \pm 6.8$ & -0.86 \\
\hline J0210.3+0105 & 021018.17 & 3.8 & +010519.86 & 2.8 & $S$ & $0.86 \pm 0.11$ & $<43.2$ & $<28.2$ & $36.0 \pm 19.6$ & -0.83 \\
\hline J0210.5-0055 & 021029.73 & 3.9 & -005515.07 & 3.1 & $S$ & $0.46 \pm 0.07$ & $<46.4$ & $<23.1$ & $29.1 \pm 14.4$ & -0.79 \\
\hline J0210.6-0822 & 021033.84 & 5.0 & -082218.97 & 4.9 & $S$ & $0.77 \pm 0.15$ & $<69.4$ & $<54.6$ & $7.3 \pm 40.2$ & -0.67 \\
\hline J0210.7-0014 & 021040.26 & 3.6 & -001440.35 & 2.2 & $S$ & $1.07 \pm 0.09$ & $<32.3$ & $<26.9$ & $83.6 \pm 32.5$ & -0.70 \\
\hline J0210.9-0633 & 021053.70 & 4.2 & -063336.84 & 2.5 & $S$ & $2.14 \pm 0.20$ & $67.8 \pm 15.0$ & $32.7 \pm 14.3$ & $84.2 \pm 6.8$ & -0.41 \\
\hline J0211.0-0329 & 021059.99 & 3.9 & -032926.17 & 3.0 & $S$ & $0.26 \pm 0.04$ & $<34.1$ & $<23.7$ & $159.5 \pm 38.0$ & -0.66 \\
\hline J0211.1-0644 & 021105.68 & 3.4 & -064442.29 & 2.0 & $S$ & $4.63 \pm 0.25$ & $41.7 \pm 5.4$ & $17.4 \pm 9.5$ & $46.8 \pm 2.7$ & -0.66 \\
\hline J0211.1-0312 & 021108.55 & 5.1 & -031245.95 & 4.8 & $S$ & $0.21 \pm 0.06$ & $<53.0$ & $<42.4$ & $166.2 \pm 65.2$ & -0.89 \\
\hline J0211.2-0735 & 021109.87 & 5.3 & -073504.72 & 4.5 & $S$ & $0.49 \pm 0.11$ & $<59.0$ & $<49.8$ & $42.0 \pm 69.3$ & -0.48 \\
\hline J0211.3-0117 & 021116.71 & 4.9 & -011755.45 & 5.3 & $S$ & $0.35 \pm 0.08$ & $<77.1$ & $<33.8$ & $26.4 \pm 13.6$ & -1.30 \\
\hline J0211.3-0250 & 021119.94 & 5.0 & -025019.19 & 4.3 & $S$ & $0.18 \pm 0.05$ & $<47.2$ & $<36.9$ & $140.5 \pm 65.1$ & -0.22 \\
\hline J0211.4-0212 & 021121.17 & 3.9 & $-02 \quad 1223.37$ & 2.8 & $S$ & $0.29 \pm 0.04$ & $<35.5$ & $<19.4$ & $37.8 \pm 21.9$ & -0.29 \\
\hline J0211.4-0118 & 021126.32 & 4.1 & -011801.90 & 3.5 & $S$ & $0.19 \pm 0.04$ & $<37.9$ & $<19.8$ & $18.8 \pm 27.5$ & -0.79 \\
\hline J0211.6-0025 & 021133.88 & 4.3 & -002554.81 & 3.2 & $S$ & $0.42 \pm 0.07$ & $<40.6$ & $<35.8$ & $118.8 \pm 86.3$ & -0.65 \\
\hline J0211.6-0008 & 021137.86 & 3.6 & -000817.29 & 2.3 & $S$ & $0.71 \pm 0.07$ & $<31.5$ & $<24.8$ & $60.9 \pm 33.0$ & -0.67 \\
\hline \multirow[t]{2}{*}{ J0211.8-0920 } & 021146.78 & 5.3 & -092050.48 & 7.2 & $M$ & $0.93 \pm 0.21$ & $65.0 \pm 40.9$ & $<50.4$ & $163.4 \pm 13.1$ & -0.70 \\
\hline & 021148.03 & 5.3 & -092034.34 & 4.1 & $C$ & $0.50 \pm 0.11$ & $<64.3$ & $<31.6$ & $53.0 \pm 18.0$ & \\
\hline J0212.0-0027 & 021201.31 & 5.7 & -002754.24 & 3.3 & $S$ & $0.24 \pm 0.06$ & $<63.5$ & $<20.3$ & $106.3 \pm 13.4$ & -0.31 \\
\hline J0212.0-0346 & 021201.66 & 4.5 & -034649.55 & 3.5 & $S$ & $0.20 \pm 0.04$ & $<40.0$ & $<32.4$ & $136.8 \pm 68.6$ & -0.47 \\
\hline J0212.1-0307 & 021205.72 & 3.4 & -030724.03 & 2.0 & $S$ & $2.30 \pm 0.13$ & $20.4 \pm 8.6$ & $<21.0$ & $37.5 \pm 7.4$ & -0.82 \\
\hline J0212.1-0516 & 021206.28 & 3.8 & -051639.01 & 2.8 & $S$ & $0.39 \pm 0.05$ & $<38.7$ & $<23.5$ & $29.0 \pm 20.1$ & -0.59 \\
\hline $\mathrm{J} 0212.2+0130$ & 021210.84 & 3.4 & +013044.02 & 2.1 & $S$ & $2.53 \pm 0.16$ & $16.8 \pm 13.2$ & $<16.2$ & $149.6 \pm 7.2$ & -0.87 \\
\hline J0212.2-0550 & 021213.77 & 3.9 & -055001.79 & 3.1 & $S$ & $0.24 \pm 0.04$ & $<38.9$ & $<16.2$ & $30.3 \pm 18.1$ & -0.62 \\
\hline \multirow[t]{2}{*}{ J0212.3-0450 } & 021216.82 & 3.8 & -045013.30 & 3.3 & $M$ & $1.01 \pm 0.11$ & $57.2 \pm 16.9$ & $<40.4$ & $11.3 \pm 8.1$ & -0.72 \\
\hline & 021216.93 & 5.7 & -045111.22 & 4.4 & $C$ & $0.29 \pm 0.07$ & $<61.8$ & $<47.3$ & $107.5 \pm 47.0$ & \\
\hline J0212.4+0100 & 021225.16 & 4.1 & +010055.98 & 4.3 & $S$ & $0.46 \pm 0.09$ & $<57.2$ & $<25.3$ & $173.1 \pm 15.0$ & -0.61 \\
\hline J0212.5-0136 & 021227.78 & 3.9 & -013613.89 & 2.6 & $S$ & $0.49 \pm 0.06$ & $<40.3$ & $<27.6$ & $74.3 \pm 22.8$ & -0.77 \\
\hline J0212.5+0118 & 021228.78 & 3.5 & +011853.08 & 2.4 & $S$ & $1.52 \pm 0.14$ & $23.5 \pm 16.4$ & $<22.7$ & $154.1 \pm 10.8$ & -0.78 \\
\hline $\mathrm{J} 0212.5+0057$ & 021230.12 & 4.9 & +005750.34 & 4.1 & $S$ & $0.23 \pm 0.06$ & $<47.8$ & $<24.4$ & $136.0 \pm 28.3$ & -0.77 \\
\hline J0212.6-0759 & 021233.38 & 5.3 & -075927.14 & 5.0 & $S$ & $0.46 \pm 0.10$ & $<78.2$ & $<36.5$ & $38.3 \pm 14.3$ & -0.63 \\
\hline J0212.6-0821 & 021235.27 & 3.7 & -082125.74 & 2.5 & $M$ & $1.82 \pm 0.16$ & $40.9 \pm 12.8$ & $25.9 \pm 15.0$ & $24.0 \pm 15.3$ & -0.64 \\
\hline & 021235.55 & 3.7 & -082033.95 & 2.5 & $C$ & $1.69 \pm 0.16$ & $34.6 \pm 13.9$ & $31.0 \pm 14.4$ & $42.4 \pm 69.0$ & \\
\hline J0212.6-0104 & 021236.86 & 4.3 & -010446.01 & 3.0 & $S$ & $0.31 \pm 0.05$ & $<42.6$ & $<28.0$ & $76.2 \pm 28.3$ & -0.73 \\
\hline J0212.7-0155 & 021241.87 & 4.0 & -015551.68 & 3.1 & $S$ & $0.20 \pm 0.04$ & $<30.0$ & $<24.1$ & $33.4 \pm 75.6$ & -0.52 \\
\hline J0212.8-0335 & 021245.83 & 3.7 & -033512.10 & 2.6 & $S$ & $0.42 \pm 0.05$ & $<35.5$ & $<23.9$ & $10.3 \pm 22.9$ & -0.75 \\
\hline J0212.8-0336 & 021246.14 & 4.2 & -033643.21 & 3.4 & $S$ & $0.37 \pm 0.06$ & $<50.6$ & $<35.3$ & $153.2 \pm 26.0$ & -0.96 \\
\hline $\mathrm{J} 0213.0+0052$ & 021257.68 & 4.1 & +00 5219.78 & 2.9 & $S$ & $0.73 \pm 0.10$ & $<51.6$ & $<29.0$ & $128.3 \pm 14.5$ & -0.74 \\
\hline J0213.1-0219 & 021305.43 & 4.2 & -021928.04 & 3.4 & $S$ & $0.23 \pm 0.04$ & $<49.2$ & $<16.7$ & $38.7 \pm 13.0$ & -0.68 \\
\hline J0213.2-0141 & 021311.91 & 3.5 & -014113.74 & 2.1 & $M$ & $0.90 \pm 0.07$ & $<26.5$ & $<23.9$ & $57.0 \pm 58.6$ & -0.80 \\
\hline & 021310.20 & 3.5 & -014057.27 & 2.2 & $C$ & $0.80 \pm 0.07$ & $<28.1$ & & & \\
\hline J0213.2-0457 & 021312.38 & 3.4 & -045719.71 & 2.0 & $S$ & $2.09 \pm 0.12$ & $26.5 \pm 8.2$ & $<21.7$ & $41.9 \pm 5.9$ & -0.60 \\
\hline J0213.2-0312 & 021314.32 & 4.3 & -031209.43 & 2.9 & $S$ & $0.61 \pm 0.08$ & $39.9 \pm 21.0$ & $<32.4$ & $62.0 \pm 11.4$ & -0.59 \\
\hline J0213.3-0719 & 021317.08 & 3.7 & -071930.68 & 2.5 & $S$ & $0.91 \pm 0.09$ & $30.4 \pm 16.1$ & $<26.9$ & $35.2 \pm 11.0$ & -0.32 \\
\hline $\mathrm{J} 0213.3+0005$ & 021317.25 & 3.8 & +00 0523.73 & 2.9 & $S$ & $0.43 \pm 0.06$ & $<38.8$ & $<19.0$ & $148.6 \pm 17.0$ & -0.94 \\
\hline J0213.3-0420 & 021319.82 & 4.1 & -042014.67 & 4.0 & $S$ & $0.31 \pm 0.06$ & $<51.8$ & $<24.6$ & $177.5 \pm 17.3$ & -0.46 \\
\hline J0213.4-0434 & 021323.79 & 3.5 & -043459.26 & 2.2 & $S$ & $0.82 \pm 0.07$ & $<32.3$ & $<24.1$ & $48.5 \pm 20.5$ & -0.85 \\
\hline J0213.4-0758 & 021324.40 & 4.2 & -075824.13 & 3.4 & $S$ & $0.62 \pm 0.10$ & $<53.5$ & $<34.7$ & $37.1 \pm 20.4$ & -0.80 \\
\hline J0213.4-0419 & 021324.77 & 3.5 & -042000.37 & 2.1 & $S$ & $2.66 \pm 0.17$ & $52.1 \pm 7.8$ & $<28.5$ & $91.6 \pm 3.2$ & -0.99 \\
\hline J0213.4-0938 & 021324.88 & 4.2 & -093800.98 & 3.9 & $S$ & $0.95 \pm 0.15$ & $34.0 \pm 27.9$ & $<42.1$ & $4.9 \pm 21.4$ & -0.88 \\
\hline
\end{tabular}


Table A.2. continued.

\begin{tabular}{|c|c|c|c|c|c|c|c|c|c|c|}
\hline Name & $\begin{array}{c}\text { RA } \\
\mathrm{J} 2000 \\
\end{array}$ & $\begin{array}{c}\sigma_{\alpha} \\
\operatorname{arcsec}\end{array}$ & $\begin{array}{c}\text { Dec } \\
\mathrm{J} 2000 \\
\end{array}$ & $\begin{array}{c}\sigma_{\delta} \\
\operatorname{arcsec}\end{array}$ & Type & $\begin{array}{c}S_{\text {comp }} \\
\mathrm{Jy}\end{array}$ & $\begin{array}{c}\text { Major axis } \\
\text { arcsec }\end{array}$ & $\begin{array}{c}\text { Minor axis } \\
\text { arcsec }\end{array}$ & $\begin{array}{c}\text { Position } \\
\text { Angle } \\
\end{array}$ & $\begin{array}{c}\text { Spectral } \\
\text { index } \alpha_{74}^{1400}\end{array}$ \\
\hline $\mathrm{J} 0213.7-0143$ & 021339.71 & 4.0 & -014302.85 & 3.3 & $S$ & $0.13 \pm 0.03$ & $<27.6$ & $<15.1$ & $18.6 \pm 45.5$ & -0.55 \\
\hline J0213.7-0345 & 021343.47 & 4.7 & -034514.89 & 4.2 & $S$ & $0.19 \pm 0.05$ & $<54.9$ & $<19.0$ & $38.4 \pm 16.1$ & -0.65 \\
\hline J0213.8+0139 & 021345.91 & 5.3 & +013948.81 & 3.9 & $S$ & $0.37 \pm 0.10$ & $<47.6$ & $<36.1$ & $78.6 \pm 57.5$ & -0.86 \\
\hline J0213.8-0256 & 021346.96 & 3.4 & -025643.04 & 2.1 & $S$ & $3.21 \pm 0.18$ & $51.9 \pm 5.8$ & $<26.2$ & $5.2 \pm 2.1$ & -0.76 \\
\hline J0213.8-0342 & 021347.40 & 4.1 & -034252.85 & 3.4 & $S$ & $0.18 \pm 0.04$ & $<38.9$ & $<12.2$ & $32.6 \pm 18.9$ & -0.57 \\
\hline J0213.9-0210 & 021351.94 & 4.3 & -021053.43 & 4.2 & $S$ & $0.14 \pm 0.04$ & $<43.5$ & $<21.8$ & $9.2 \pm 28.1$ & -0.76 \\
\hline J0213.9-0253 & 021356.37 & 4.7 & -025326.61 & 4.0 & $S$ & $0.25 \pm 0.06$ & $<46.0$ & - & & -0.68 \\
\hline J0214.3-0437 & 021416.25 & 5.0 & -043710.06 & 3.6 & $S$ & $0.39 \pm 0.07$ & $<63.1$ & $<39.6$ & $114.1 \pm 20.7$ & -0.81 \\
\hline \multirow[t]{2}{*}{ J0214.3-0153 } & 021416.55 & 3.5 & -015321.41 & 2.2 & $M$ & $0.80 \pm 0.07$ & $<32.2$ & $<24.7$ & $127.8 \pm 22.6$ & -0.60 \\
\hline & 021414.49 & 4.0 & -015258.77 & 3.0 & C & $0.13 \pm 0.03$ & $<21.8$ & & & \\
\hline J0214.3-0422 & 021420.36 & 3.7 & -042220.04 & 2.7 & $S$ & $0.28 \pm 0.04$ & $<33.8$ & $<16.2$ & $22.9 \pm 18.0$ & -0.77 \\
\hline J0214.4+0143 & 021422.60 & 5.2 & +014326.52 & 4.9 & $S$ & $0.59 \pm 0.14$ & $<60.8$ & $<46.5$ & $158.3 \pm 47.0$ & -0.85 \\
\hline J0214.4-0555 & 021425.45 & 3.9 & -055544.39 & 3.0 & $S$ & $0.38 \pm 0.05$ & $<44.5$ & $<22.5$ & $30.5 \pm 15.0$ & -0.58 \\
\hline J0214.5-0720 & 021427.24 & 3.6 & -072009.43 & 2.4 & $S$ & $1.13 \pm 0.10$ & $40.0 \pm 12.1$ & $<24.9$ & $37.9 \pm 6.2$ & -0.80 \\
\hline J0214.6-0603 & 021436.38 & 3.5 & -060344.79 & 2.2 & $S$ & $0.90 \pm 0.07$ & $26.1 \pm 12.6$ & $<16.4$ & $46.7 \pm 6.6$ & -0.71 \\
\hline J0214.6+0042 & 021437.02 & 4.0 & +004230.60 & 3.9 & $S$ & $0.74 \pm 0.12$ & $35.6 \pm 26.8$ & $<31.9$ & $9.3 \pm 12.9$ & -0.58 \\
\hline J0214.7-0004 & 021444.58 & 6.1 & -000427.89 & 6.0 & $S$ & $0.67 \pm 0.13$ & $75.7 \pm 38.6$ & $<36.8$ & $41.1 \pm 7.6$ & -0.89 \\
\hline J0214.8-0541 & 021449.96 & 4.6 & -054157.19 & 4.1 & $S$ & $0.42 \pm 0.08$ & $<56.7$ & $<46.0$ & $8.4 \pm 46.5$ & -0.57 \\
\hline J0215.0-0458* & 021459.25 & 4.1 & -045807.65 & 3.6 & $S$ & $0.17 \pm 0.04$ & $<40.1$ & $<19.8$ & $21.0 \pm 24.6$ & -0.39 \\
\hline J0215.0-0453* & 021500.17 & 3.5 & -045350.48 & 2.6 & $S$ & $1.19 \pm 0.10$ & $52.0 \pm 11.6$ & $<28.8$ & $170.5 \pm 4.8$ & -0.74 \\
\hline J0215.0+0114 & 021501.51 & 3.4 & +011448.28 & 2.0 & $S$ & $3.28 \pm 0.20$ & $22.5 \pm 9.7$ & $<24.5$ & $136.2 \pm 11.1$ & -0.93 \\
\hline J0215.1-0600 & 021505.78 & 3.5 & -060050.90 & 2.2 & $S$ & $0.97 \pm 0.08$ & $20.8 \pm 14.9$ & $<21.5$ & $40.5 \pm 10.5$ & -0.76 \\
\hline J0215.1-0702 & 021508.03 & 3.4 & -070204.40 & 2.1 & $S$ & $2.11 \pm 0.13$ & $39.5 \pm 7.5$ & $<24.0$ & $45.6 \pm 3.9$ & -0.67 \\
\hline J0215.2-0135 & 021510.00 & 3.4 & -013527.27 & 2.0 & $S$ & $1.71 \pm 0.10$ & $25.2 \pm 9.0$ & $<17.9$ & $60.7 \pm 5.3$ & -1.04 \\
\hline J0215.2-0343* & 021511.52 & 3.5 & -034312.08 & 2.2 & $S$ & $0.68 \pm 0.05$ & $<32.0$ & $<14.2$ & $42.5 \pm 9.1$ & -0.43 \\
\hline \multirow[t]{2}{*}{$\mathrm{J} 0215.3+0030$} & 021515.79 & 3.8 & +003032.18 & 3.0 & $M$ & $0.52 \pm 0.08$ & $<39.5$ & $<20.7$ & $178.8 \pm 18.1$ & -0.67 \\
\hline & 021516.80 & 4.0 & +00 3034.04 & 3.6 & $C$ & $0.32 \pm 0.06$ & $<49.1$ & $<3.1$ & $154.2 \pm 10.8$ & \\
\hline J0215.3-0153 & 021517.14 & 3.5 & -015332.72 & 2.1 & $S$ & $0.75 \pm 0.06$ & $<20.7$ & $<17.0$ & $63.3 \pm 46.0$ & -0.81 \\
\hline $\mathrm{J} 0215.3+0050$ & 021517.38 & 4.6 & +005021.82 & 4.4 & $S$ & $0.33 \pm 0.08$ & $<52.8$ & $<28.2$ & $155.8 \pm 24.2$ & -0.66 \\
\hline $\mathrm{J} 0215.3+0040$ & 021519.03 & 3.5 & +004044.88 & 2.3 & $S$ & $1.19 \pm 0.11$ & $<33.2$ & $<22.5$ & $144.5 \pm 17.7$ & -0.58 \\
\hline J0215.6-0756 & 021534.22 & 4.3 & -075608.22 & 4.0 & $S$ & $0.35 \pm 0.07$ & $<51.8$ & $<36.7$ & $7.7 \pm 32.0$ & -0.59 \\
\hline J0215.6-0321* & 021534.47 & 3.8 & -032200.00 & 2.9 & $S$ & $0.67 \pm 0.08$ & $41.0 \pm 17.1$ & $<26.8$ & $31.0 \pm 8.2$ & -0.61 \\
\hline J0215.6-0711 & 021534.98 & 4.4 & -071100.46 & 3.2 & $S$ & $0.63 \pm 0.09$ & $38.2 \pm 23.2$ & $<39.4$ & $57.0 \pm 16.9$ & -0.54 \\
\hline J0215.6-0206 & 021537.39 & 3.5 & -020605.80 & 2.2 & $S$ & $0.62 \pm 0.06$ & $<25.2$ & $<18.1$ & $53.6 \pm 28.9$ & -0.93 \\
\hline J0215.7-0056 & 021539.61 & 3.7 & -005659.28 & 2.4 & $S$ & $0.62 \pm 0.07$ & $<36.1$ & $<23.1$ & $100.3 \pm 18.7$ & -0.97 \\
\hline J0215.7-0222* & 021541.89 & 3.4 & -022255.03 & 2.0 & $S$ & $5.29 \pm 0.27$ & $19.0 \pm 7.3$ & $<21.1$ & $19.3 \pm 6.1$ & -0.81 \\
\hline J0215.7-0057 & 021544.17 & 4.1 & -005711.24 & 2.6 & $S$ & $0.45 \pm 0.06$ & $<41.8$ & $<23.5$ & $83.2 \pm 18.0$ & -0.95 \\
\hline J0215.7+0133 & 021544.46 & 5.0 & +013318.38 & 4.4 & $S$ & $0.50 \pm 0.12$ & $<58.6$ & $<34.0$ & $139.7 \pm 25.1$ & -0.56 \\
\hline J0215.8-0142 & 021544.99 & 3.5 & -014250.53 & 2.1 & $S$ & $0.78 \pm 0.06$ & $<20.5$ & $<10.2$ & $76.1 \pm 17.6$ & -0.60 \\
\hline J0215.9-0359* & 021552.26 & 4.3 & -035951.32 & 3.5 & $S$ & $0.11 \pm 0.03$ & $<28.7$ & & $\ldots$ & -0.25 \\
\hline J0215.9+0025 & 021556.51 & 5.2 & +002523.27 & 4.0 & $S$ & $0.33 \pm 0.08$ & $<57.4$ & $<31.1$ & $53.6 \pm 23.0$ & -0.70 \\
\hline J0216.0-0825 & 021557.56 & 5.2 & -082515.24 & 5.7 & $S$ & $0.43 \pm 0.10$ & $<75.2$ & $<49.4$ & $166.9 \pm 27.2$ & -0.58 \\
\hline \multirow[t]{2}{*}{ J0216.0-0350* } & 021558.66 & 7.7 & -035027.56 & 3.8 & $M$ & $0.30 \pm 0.07$ & $51.3 \pm 46.3$ & $<35.1$ & $84.4 \pm 10.9$ & -0.82 \\
\hline & 021557.81 & 4.3 & -035044.79 & 3.8 & $C$ & $0.11 \pm 0.03$ & $<38.1$ & $<16.9$ & $153.6 \pm 28.5$ & \\
\hline J0216.0-0344* & 021559.79 & 3.5 & -034421.49 & 2.2 & $S$ & $0.92 \pm 0.07$ & $<31.3$ & $<25.0$ & $59.9 \pm 23.2$ & -0.70 \\
\hline J0216.2-0011 & 021613.94 & 3.9 & -001139.46 & 3.0 & $S$ & $0.42 \pm 0.06$ & $<36.9$ & $<29.9$ & $21.0 \pm 51.2$ & -0.82 \\
\hline $\mathrm{J} 0216.3+0008$ & 021616.54 & 3.4 & +000858.67 & 2.1 & $S$ & $1.12 \pm 0.09$ & $<24.6$ & $<15.9$ & $166.7 \pm 17.6$ & -0.60 \\
\hline J0216.3-0010 & 021619.84 & 3.7 & -001059.43 & 2.5 & $S$ & $0.39 \pm 0.05$ & $<23.6$ & & & -0.58 \\
\hline \multirow[t]{2}{*}{ J0216.3-0245* } & 021620.11 & 4.0 & -024530.82 & 2.9 & $S$ & $0.52 \pm 0.07$ & $<44.1$ & $<33.4$ & $135.4 \pm 30.0$ & -0.37 \\
\hline & 021624.64 & 5.4 & -024518.69 & 3.9 & $S$ & $0.43 \pm 0.09$ & $<64.6$ & $<47.6$ & $83.0 \pm 33.4$ & -0.37 \\
\hline J0216.4-0917 & 021623.69 & 4.9 & -091724.86 & 3.8 & $S$ & $0.36 \pm 0.08$ & $<51.8$ & $<35.2$ & $55.8 \pm 33.1$ & -0.82 \\
\hline J0216.5-0623 & 021629.22 & 4.4 & -062351.55 & 4.3 & $S$ & $0.18 \pm 0.04$ & $<49.4$ & $<25.9$ & $15.9 \pm 24.6$ & -0.54 \\
\hline J0216.6-0024 & 021636.81 & 4.8 & -002454.03 & 4.1 & $S$ & $0.33 \pm 0.07$ & $<50.6$ & $<37.6$ & $34.2 \pm 44.7$ & -0.67 \\
\hline J0216.7-0953 & 021640.20 & 4.4 & -095343.33 & 3.6 & $S$ & $0.66 \pm 0.12$ & $<51.2$ & $<39.2$ & $29.9 \pm 37.3$ & -0.56 \\
\hline J0216.7-0444* & 021640.93 & 4.7 & -044405.50 & 3.9 & $S$ & $0.19 \pm 0.04$ & $<49.8$ & $<27.3$ & $42.1 \pm 24.4$ & -0.25 \\
\hline J0216.7-0613 & 021642.23 & 3.8 & -061345.08 & 2.7 & $S$ & $0.43 \pm 0.06$ & $<40.6$ & $<28.1$ & $40.8 \pm 23.0$ & -0.76 \\
\hline J0216.8-0337* & 021645.73 & 4.0 & -033718.34 & 3.0 & $S$ & $0.27 \pm 0.05$ & $<38.5$ & $<25.3$ & $44.9 \pm 29.2$ & -0.57 \\
\hline J0216.8-0237* & 021646.79 & 5.5 & -023718.64 & 4.5 & $S$ & $0.24 \pm 0.07$ & $<50.7$ & & 年- & -0.72 \\
\hline \multirow[t]{2}{*}{ J0217.0-0449* } & 021658.91 & 4.8 & -045000.78 & 4.2 & $S$ & $0.46 \pm 0.08$ & $<67.1$ & $<50.2$ & $34.4 \pm 30.5$ & -1.52 \\
\hline & 021659.09 & 4.8 & -044843.92 & 6.3 & $S$ & $0.46 \pm 0.09$ & $63.1 \pm 35.4$ & $<44.0$ & $16.1 \pm 11.1$ & -1.52 \\
\hline J0217.0-0155 & 021659.37 & 4.3 & -015512.14 & 3.0 & $S$ & $0.27 \pm 0.05$ & $<41.8$ & $<23.2$ & $61.9 \pm 23.7$ & -0.67 \\
\hline J0217.0-0954 & 021702.24 & 3.8 & -095450.12 & 2.8 & $S$ & $1.29 \pm 0.14$ & $38.5 \pm 16.7$ & $<30.8$ & $37.4 \pm 10.3$ & -0.82 \\
\hline J0217.0-0603 & 021702.58 & 3.5 & -060327.39 & 2.2 & $S$ & $0.69 \pm 0.06$ & $<35.8$ & $<20.5$ & $47.1 \pm 10.8$ & -1.44 \\
\hline \multirow[t]{2}{*}{$\mathrm{J} 0217.1-0210^{*}$} & 021703.15 & 4.1 & -021051.76 & 2.4 & $M$ & $0.37 \pm 0.05$ & $<47.0$ & $<10.1$ & $87.0 \pm 8.3$ & -0.58 \\
\hline & 021702.72 & 3.7 & -021037.61 & 2.4 & $C$ & $0.19 \pm 0.03$ & $<14.6$ & & $80.5 \pm 43.4$ & \\
\hline J0217.1-0820 & 021703.16 & 3.8 & -082046.04 & 2.8 & $S$ & $0.99 \pm 0.11$ & $35.2 \pm 17.2$ & $<37.5$ & $28.5 \pm 17.5$ & -0.28 \\
\hline J0217.1-0422* & 021705.70 & 4.0 & -042254.75 & 2.9 & $S$ & $0.40 \pm 0.06$ & $<49.4$ & $<23.9$ & $48.9 \pm 12.4$ & -0.59 \\
\hline J0217.3-0825 & 021718.19 & 4.1 & -082517.10 & 4.2 & $S$ & $0.56 \pm 0.09$ & $40.8 \pm 27.2$ & $<33.2$ & $8.5 \pm 12.1$ & -0.82 \\
\hline J0217.3-0326* & 021719.73 & 4.2 & -032603.67 & 3.0 & $S$ & $0.32 \pm 0.05$ & $<41.2$ & $<34.0$ & $90.8 \pm 52.3$ & -0.79 \\
\hline J0217.3+0155 & 021720.64 & 3.4 & +015523.45 & 2.1 & $S$ & $3.68 \pm 0.25$ & $31.8 \pm 9.4$ & $<23.9$ & $152.6 \pm 6.4$ & -0.98 \\
\hline J0217.4-0339* & 021721.74 & 4.6 & -033948.20 & 3.3 & $S$ & $0.22 \pm 0.05$ & $<43.7$ & $<29.2$ & $62.0 \pm 33.9$ & -0.48 \\
\hline J0217.4-0618 & 021722.81 & 3.7 & -061831.15 & 2.7 & $S$ & $0.19 \pm 0.03$ & $<31.3$ & 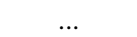 & $29.2 \pm 12.4$ & -0.49 \\
\hline J0217.4-0200 & 021724.50 & 3.7 & -020006.19 & 2.6 & $S$ & $0.16 \pm 0.03$ & $<16.7$ & & $1.6 \pm 47.7$ & -0.64 \\
\hline J0217.4-0015 & 021725.10 & 4.7 & -001547.82 & 4.2 & $S$ & $0.27 \pm 0.07$ & $<45.9$ & $<34.6$ & $156.7 \pm 54.7$ & -0.75 \\
\hline
\end{tabular}


Table A.2. continued.

\begin{tabular}{|c|c|c|c|c|c|c|c|c|c|c|}
\hline Name & $\begin{array}{c}\text { RA } \\
\text { J2000 } \\
\end{array}$ & $\begin{array}{c}\sigma_{\alpha} \\
\operatorname{arcsec} \\
\end{array}$ & $\begin{array}{c}\text { Dec } \\
\text { J2000 } \\
\end{array}$ & $\begin{array}{c}\sigma_{\delta} \\
\operatorname{arcsec}\end{array}$ & Type & $\begin{array}{c}S_{\text {comp }} \\
\text { Jy }\end{array}$ & $\begin{array}{c}\text { Major axis } \\
\text { arcsec }\end{array}$ & $\begin{array}{c}\text { Minor axis } \\
\text { arcsec }\end{array}$ & $\begin{array}{c}\text { Position } \\
\text { Angle }\end{array}$ & $\begin{array}{c}\text { Spectral } \\
\text { index } \alpha_{74}^{1400}\end{array}$ \\
\hline $\mathrm{J} 0217.5-0052$ & 021728.41 & 3.4 & -005229.80 & 2.0 & $S$ & $1.23 \pm 0.08$ & $<19.3$ & & & -0.58 \\
\hline J0217.5-0725 & 021728.80 & 5.0 & -072552.52 & 4.6 & $S$ & $0.25 \pm 0.06$ & $<56.1$ & $<42.9$ & $30.6 \pm 48.2$ & -0.76 \\
\hline J0217.5-0047 & 021732.26 & 3.6 & -004701.31 & 2.3 & $S$ & $0.71 \pm 0.07$ & $<37.3$ & $<18.0$ & $56.2 \pm 11.2$ & -0.65 \\
\hline J0217.6-0313* & 021733.98 & 4.1 & -031310.13 & 3.3 & $S$ & $0.24 \pm 0.04$ & $<49.2$ & $<11.1$ & $35.2 \pm 11.0$ & -0.67 \\
\hline J0217.7-0917 & 021740.92 & 6.1 & -09 1735.75 & 4.4 & $S$ & $0.65 \pm 0.14$ & $43.9 \pm 37.7$ & $<48.8$ & $65.3 \pm 19.2$ & -0.80 \\
\hline J0217.7-0541* & 021741.17 & 4.0 & -054155.48 & 2.8 & $S$ & $0.54 \pm 0.07$ & $<46.2$ & $<39.5$ & $89.7 \pm 45.9$ & -0.72 \\
\hline J0217.8-0157 & 021746.14 & 3.8 & -015719.29 & 2.6 & $S$ & $0.34 \pm 0.05$ & $<27.7$ & $<21.3$ & $51.7 \pm 51.2$ & -0.78 \\
\hline \multirow[t]{2}{*}{ J0217.8-0030 } & 021746.35 & 3.4 & -003033.86 & 2.2 & $M$ & $4.54 \pm 0.26$ & $76.2 \pm 6.7$ & $<21.2$ & $155.1 \pm 1.2$ & -0.93 \\
\hline & 021744.51 & 3.6 & -002945.96 & 2.2 & $C$ & $0.32 \pm 0.04$ & $<19.8$ & $\ldots$ & $64.6 \pm 10.4$ & \\
\hline $\mathrm{J} 0217.8+0008$ & 021748.21 & 3.6 & +00 0858.74 & 2.4 & $S$ & $1.00 \pm 0.10$ & $<36.0$ & $<29.0$ & $58.5 \pm 29.0$ & -1.06 \\
\hline J0217.8+0144 & 021748.45 & 3.4 & +014448.08 & 2.1 & $S$ & $2.84 \pm 0.19$ & $22.5 \pm 11.0$ & $<22.8$ & $153.0 \pm 9.8$ & -0.44 \\
\hline J0217.9-0036 & 021756.87 & 4.1 & -003606.61 & 3.3 & $S$ & $0.26 \pm 0.05$ & $<37.0$ & $<19.3$ & $30.4 \pm 26.7$ & -1.13 \\
\hline J0218.0-0918 & 021758.18 & 4.3 & -091818.91 & 4.2 & $S$ & $0.75 \pm 0.12$ & $42.7 \pm 26.6$ & $<38.3$ & $21.5 \pm 14.0$ & -0.78 \\
\hline J0218.2-0301* & 021811.79 & 4.2 & -030128.65 & 3.2 & $S$ & $0.33 \pm 0.06$ & $<39.6$ & $<33.9$ & $127.6 \pm 72.4$ & -0.79 \\
\hline J0218.3-0121 & 021815.01 & 4.6 & -012138.20 & 4.7 & $S$ & $0.19 \pm 0.05$ & $<48.9$ & $<30.2$ & $6.7 \pm 35.9$ & -0.67 \\
\hline J0218.3-0446* & 021818.03 & 3.7 & -044608.13 & 2.9 & $S$ & $0.30 \pm 0.04$ & $<35.5$ & $<20.8$ & $0.8 \pm 22.9$ & -0.53 \\
\hline J0218.5-0454* & 021827.28 & 3.5 & -045438.95 & 2.2 & $S$ & $0.80 \pm 0.06$ & $<34.1$ & $<16.3$ & $41.8 \pm 8.2$ & -0.77 \\
\hline J0218.6-0923 & 021834.74 & 4.5 & -09 2338.45 & 4.7 & $S$ & $0.50 \pm 0.10$ & $<72.5$ & $<28.8$ & $26.2 \pm 11.3$ & -0.39 \\
\hline J0218.6-0725 & 021838.06 & 3.6 & -072529.04 & 2.3 & $S$ & $0.98 \pm 0.08$ & $35.1 \pm 12.6$ & $<24.2$ & $48.5 \pm 7.3$ & -0.64 \\
\hline J0218.7-0441* & 021839.62 & 3.5 & -044150.61 & 2.2 & $S$ & $0.65 \pm 0.06$ & $<29.9$ & $<25.0$ & $38.2 \pm 36.0$ & -0.84 \\
\hline J0218.7-0015 & 021839.85 & 4.7 & -001507.84 & 3.7 & $S$ & $0.25 \pm 0.06$ & $<41.3$ & $<32.8$ & $54.2 \pm 69.0$ & -0.57 \\
\hline J0218.8-0836 & 021845.56 & 4.2 & -083657.88 & 3.4 & $S$ & $0.21 \pm 0.05$ & $<32.9$ & & & -0.20 \\
\hline J0218.8-0211* & 021850.73 & 3.4 & -021116.64 & 2.0 & $S$ & $1.79 \pm 0.11$ & $<24.0$ & $<16.8$ & $46.5 \pm 12.5$ & -0.68 \\
\hline J0218.9-0812 & 021854.95 & 4.1 & -081254.21 & 3.5 & $S$ & $0.71 \pm 0.10$ & $36.9 \pm 22.3$ & $<44.9$ & $16.7 \pm 25.4$ & -0.42 \\
\hline J0219.0-0149 & 021857.06 & 3.5 & -014911.44 & 2.2 & $S$ & $0.81 \pm 0.07$ & $<25.0$ & $<18.5$ & $71.8 \pm 27.3$ & -0.52 \\
\hline J0219.0-0155 & 021857.62 & 3.6 & -015533.98 & 2.3 & $S$ & $0.43 \pm 0.05$ & $<25.9$ & $<9.5$ & $66.7 \pm 18.4$ & -0.65 \\
\hline J0219.1+0120 & 021906.59 & 3.5 & +012056.05 & 2.2 & $S$ & $2.04 \pm 0.16$ & $26.9 \pm 12.4$ & $<24.3$ & $167.3 \pm 9.7$ & -0.49 \\
\hline J0219.1-0252* & 021907.28 & 3.4 & -025251.85 & 2.1 & $S$ & $0.89 \pm 0.06$ & $<23.5$ & $<16.4$ & $60.0 \pm 19.9$ & -0.43 \\
\hline \multirow[t]{2}{*}{ J0219.1-0357* } & 021908.39 & 4.8 & -035750.95 & 3.6 & $M$ & $0.19 \pm 0.04$ & $<45.4$ & $<32.2$ & $58.0 \pm 42.2$ & -0.59 \\
\hline & 021908.82 & 4.1 & -035806.53 & 3.3 & $C$ & $0.08 \pm 0.02$ & $<27.0$ & & $36.3 \pm 32.3$ & \\
\hline J0219.2-0344* & 021911.19 & 4.8 & -034431.78 & 3.6 & $S$ & $0.14 \pm 0.04$ & $<41.0$ & $<28.0$ & $69.8 \pm 46.7$ & -0.46 \\
\hline J0219.3-0152 & 021917.92 & 4.7 & -015249.56 & 3.7 & $S$ & $0.32 \pm 0.07$ & $<48.8$ & $<29.9$ & $129.4 \pm 28.3$ & -0.71 \\
\hline J0219.3-0552* & 021918.21 & 4.9 & -055243.70 & 4.3 & $S$ & $0.29 \pm 0.06$ & $<67.5$ & $<26.2$ & $42.1 \pm 12.8$ & -0.63 \\
\hline J0219.3-0244* & 021920.05 & 4.6 & -024411.83 & 3.5 & $S$ & $0.38 \pm 0.07$ & $<55.4$ & $<31.0$ & $48.7 \pm 18.8$ & -0.62 \\
\hline J0219.3-0051 & 021920.20 & 4.8 & -005136.57 & 3.3 & $S$ & $0.21 \pm 0.05$ & $<44.6$ & $<15.7$ & $118.3 \pm 19.7$ & -0.33 \\
\hline J0219.5-0539* & 021928.28 & 3.4 & -053947.63 & 2.0 & $S$ & $3.89 \pm 0.20$ & $20.6 \pm 7.1$ & $<19.5$ & $49.6 \pm 4.5$ & -0.76 \\
\hline J0219.5+0024 & 021928.49 & 5.0 & +002437.24 & 3.4 & $S$ & $0.45 \pm 0.09$ & $<65.3$ & $<18.6$ & $58.6 \pm 9.8$ & -0.69 \\
\hline J0219.5-0251* & 021929.94 & 3.5 & -025112.60 & 2.1 & $S$ & $0.95 \pm 0.07$ & $<31.0$ & $<15.2$ & $95.8 \pm 8.7$ & -0.72 \\
\hline \multirow[t]{2}{*}{ J0219.5-0058 } & 021931.50 & 3.5 & -005826.65 & 2.2 & $M$ & $0.51 \pm 0.05$ & $<20.9$ & $<4.1$ & $129.9 \pm 19.3$ & -0.83 \\
\hline & 021933.03 & 3.8 & -005832.82 & 3.8 & $C$ & $0.49 \pm 0.08$ & $<58.4$ & $<19.9$ & $172.8 \pm 9.8$ & \\
\hline J0219.5-0747 & 021932.26 & 4.7 & -074739.59 & 3.5 & $S$ & $0.54 \pm 0.09$ & $42.8 \pm 26.0$ & $<37.9$ & $54.6 \pm 13.7$ & -0.47 \\
\hline J0219.6-0944 & 021934.03 & 4.9 & -094402.27 & 4.5 & $S$ & $0.59 \pm 0.12$ & $<62.0$ & $<49.6$ & $21.9 \pm 46.6$ & -1.01 \\
\hline J0219.7-0400 & 021941.51 & 6.3 & -040030.39 & 9.5 & $S$ & $1.34 \pm 0.20$ & $172.2 \pm 50.1$ & $31.3 \pm 25.6$ & $153.4 \pm 3.5$ & -1.15 \\
\hline J0219.8+0001 & 021944.99 & 3.7 & +00 0157.18 & 2.8 & $S$ & $0.41 \pm 0.06$ & $<31.7$ & $<16.5$ & $4.8 \pm 23.3$ & -0.50 \\
\hline J0219.9-0518* & 021952.98 & 3.9 & -051828.29 & 4.0 & $S$ & $0.20 \pm 0.04$ & $<53.0$ & $<10.7$ & $166.6 \pm 11.1$ & -0.76 \\
\hline J0219.9-0732 & 021955.34 & 4.3 & -073201.63 & 3.7 & $S$ & $0.34 \pm 0.06$ & $<51.6$ & $<35.1$ & $24.9 \pm 27.1$ & -0.62 \\
\hline J0220.0-0447* & 021957.58 & 3.9 & -044749.90 & 3.3 & $S$ & $0.29 \pm 0.05$ & $<44.8$ & $<25.9$ & $11.7 \pm 20.4$ & -0.65 \\
\hline \multirow{2}{*}{$\mathrm{J} 0220.0+0155$} & 021958.05 & 3.5 & +015532.44 & 2.3 & $M$ & $2.21 \pm 0.19$ & $24.7 \pm 14.6$ & $<32.3$ & $176.4 \pm 24.3$ & -0.60 \\
\hline & 021958.61 & 3.6 & +015608.31 & 2.4 & $C$ & $1.11 \pm 0.12$ & $<27.5$ & & & \\
\hline J0220.0-0155 & 021959.06 & 3.7 & -015537.06 & 2.5 & $S$ & $0.70 \pm 0.09$ & $<32.4$ & $<19.4$ & $35.1 \pm 21.2$ & -0.73 \\
\hline J0220.0-0143 & 022002.01 & 4.0 & -014350.48 & 3.1 & $S$ & $0.42 \pm 0.07$ & $<41.3$ & $<15.6$ & $143.8 \pm 16.1$ & -0.70 \\
\hline J0220.0-0000 & 022002.73 & 5.0 & -000022.08 & 4.3 & $S$ & $0.28 \pm 0.07$ & $<48.4$ & $<37.1$ & $42.6 \pm 58.0$ & -0.63 \\
\hline J0220.2-0723 & 022008.95 & 4.0 & -072349.10 & 4.5 & $S$ & $0.48 \pm 0.08$ & $51.7 \pm 25.5$ & $<31.6$ & $176.6 \pm 8.9$ & -0.78 \\
\hline J0220.5+0152 & 022031.74 & 4.0 & +015238.03 & 3.1 & $S$ & $0.54 \pm 0.10$ & $<32.8$ & $<24.0$ & $5.5 \pm 48.8$ & -0.96 \\
\hline J0220.5+0027 & 022032.50 & 3.8 & +00 2759.43 & 2.8 & $S$ & $0.69 \pm 0.09$ & $<37.9$ & $<26.0$ & $154.5 \pm 24.6$ & -0.60 \\
\hline J0220.6+0232 & 022036.58 & 4.4 & +023212.69 & 3.8 & $S$ & $0.74 \pm 0.15$ & $<52.3$ & $<25.9$ & $148.0 \pm 18.4$ & -0.84 \\
\hline J0220.7-0149 & 022043.51 & 3.7 & -014958.16 & 2.5 & $S$ & $0.51 \pm 0.07$ & $<18.5$ & $\ldots$ & $\ldots$ & -0.40 \\
\hline J0220.8-0333* & 022049.38 & 3.6 & -033317.48 & 2.4 & $S$ & $0.32 \pm 0.04$ & $<20.6$ & $\ldots$ & $\ldots$ & -0.37 \\
\hline J0220.9-0601* & 022052.46 & 5.3 & -060115.63 & 3.2 & $S$ & $0.25 \pm 0.05$ & $<59.0$ & $<28.0$ & $89.7 \pm 16.5$ & -0.72 \\
\hline J0220.9-0156* & 022054.15 & 3.4 & -015653.84 & 2.0 & $S$ & $40.51 \pm 2.03$ & $<20.0$ & $<15.0$ & $43.3 \pm 1.4$ & -0.83 \\
\hline J0220.9-0725 & 022054.82 & 3.7 & -072544.85 & 2.8 & $S$ & $0.42 \pm 0.05$ & $<46.8$ & $<18.2$ & $29.5 \pm 9.9$ & -0.50 \\
\hline J0220.9-0348* & 022055.71 & 3.4 & -034834.07 & 2.0 & $S$ & $1.17 \pm 0.07$ & $<24.3$ & $<14.6$ & $57.4 \pm 10.3$ & -0.90 \\
\hline J0220.9-0219* & 022056.06 & 3.8 & -021902.27 & 3.5 & $S$ & $0.33 \pm 0.06$ & $<46.7$ & $\cdots$ & $24.5 \pm 8.4$ & -1.42 \\
\hline $\mathrm{J} 0221.0+0231$ & 022102.39 & 4.9 & +023156.80 & 4.0 & $S$ & $0.56 \pm 0.14$ & $<42.8$ & $\ldots$ & $\ldots$ & -0.76 \\
\hline J0221.1+0059 & 022103.28 & 3.4 & +005938.05 & 2.0 & $S$ & $2.39 \pm 0.14$ & $<25.1$ & $<14.9$ & $177.6 \pm 8.5$ & -0.68 \\
\hline J0221.1-0246* & 022103.50 & 4.3 & -024630.71 & 3.9 & $S$ & $0.32 \pm 0.07$ & $<47.0$ & $<31.5$ & $166.7 \pm 32.6$ & -0.67 \\
\hline J0221.1-0931 & 022107.31 & 3.8 & -093146.69 & 2.9 & $S$ & $0.65 \pm 0.09$ & $<45.2$ & $<23.8$ & $31.8 \pm 14.5$ & -0.75 \\
\hline J0221.2-0146 & 022113.38 & 3.6 & -014604.81 & 2.8 & $S$ & $1.56 \pm 0.16$ & $41.8 \pm 14.9$ & $<22.4$ & $9.1 \pm 6.3$ & -0.82 \\
\hline \multirow[t]{3}{*}{$\mathrm{J} 0221.3-0015$} & 022115.60 & 3.9 & -001557.30 & 4.1 & $M$ & $0.52 \pm 0.07$ & $59.9 \pm 23.0$ & 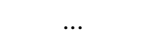 & $24.8 \pm 3.4$ & -0.73 \\
\hline & 022114.21 & 3.8 & -001604.41 & 2.7 & $C$ & $0.20 \pm 0.04$ & $<20.7$ & & $33.9 \pm 34.2$ & \\
\hline & 022116.98 & 3.7 & -001547.96 & 2.5 & $C$ & $0.16 \pm 0.03$ & $<14.3$ & & $33.8 \pm 22.2$ & \\
\hline J0221.3-0255* & 022116.22 & 3.6 & -025549.68 & 2.4 & $S$ & $0.42 \pm 0.05$ & $<27.1$ & $<12.3$ & $50.9 \pm 19.2$ & -0.49 \\
\hline J0221.3+0141 & 022118.76 & 3.4 & +014108.81 & 2.0 & $S$ & $5.09 \pm 0.30$ & $37.5 \pm 6.7$ & $<26.7$ & $39.2 \pm 4.2$ & -1.14 \\
\hline J0221.3-0305* & 022119.55 & 4.7 & -030547.27 & 4.0 & $S$ & $0.28 \pm 0.06$ & $<49.0$ & $<37.5$ & $33.3 \pm 48.4$ & -0.97 \\
\hline
\end{tabular}


Table A.2. continued.

\begin{tabular}{|c|c|c|c|c|c|c|c|c|c|c|}
\hline Name & $\begin{array}{c}\text { RA } \\
\text { J2000 }\end{array}$ & $\begin{array}{c}\sigma_{\alpha} \\
\operatorname{arcsec}\end{array}$ & $\begin{array}{c}\text { Dec } \\
\text { J2000 }\end{array}$ & $\begin{array}{c}\sigma_{\delta} \\
\operatorname{arcsec}\end{array}$ & Type & $\begin{array}{c}S_{\text {comp }} \\
\text { Jy }\end{array}$ & $\begin{array}{c}\text { Major axis } \\
\text { arcsec }\end{array}$ & $\begin{array}{c}\text { Minor axis } \\
\text { arcsec }\end{array}$ & $\begin{array}{l}\text { Position } \\
\text { Angle }\end{array}$ & $\begin{array}{c}\text { Spectral } \\
\text { index } \alpha_{74}^{1400}\end{array}$ \\
\hline J0221.7-0451* & 022142.11 & 3.6 & -045110.45 & 2.4 & $S$ & $0.63 \pm 0.06$ & $<36.2$ & $<28.8$ & $58.8 \pm 28.5$ & -0.83 \\
\hline J0221.7-0413* & 022143.07 & 3.4 & -041344.86 & 2.0 & $S$ & $2.41 \pm 0.13$ & $<24.2$ & $<17.7$ & $41.4 \pm 8.0$ & -0.51 \\
\hline J0221.8-0925 & 022150.83 & 4.6 & -092505.03 & 4.7 & $S$ & $0.29 \pm 0.07$ & $<66.2$ & $<19.2$ & $31.7 \pm 11.7$ & -0.53 \\
\hline J0222.0-0240* & 022200.59 & 3.6 & -024008.69 & 2.2 & $S$ & $1.30 \pm 0.11$ & $43.0 \pm 11.5$ & $<15.9$ & $122.2 \pm 4.1$ & -0.72 \\
\hline $\mathrm{J} 0222.1-0620$ & 022203.21 & 4.5 & -062003.52 & 4.1 & $S$ & $0.16 \pm 0.04$ & $<46.4$ & $<27.1$ & $152.2 \pm 31.0$ & -0.66 \\
\hline $\mathrm{J} 0222.1+0101$ & 022203.43 & 3.9 & +010105.89 & 3.6 & $S$ & $0.36 \pm 0.07$ & $<47.6$ & $<11.0$ & $159.5 \pm 12.0$ & -0.61 \\
\hline J0222.1+0137 & 022205.09 & 3.4 & +013721.43 & 2.1 & $S$ & $2.14 \pm 0.14$ & $<29.6$ & $<19.8$ & $165.6 \pm 11.4$ & -0.71 \\
\hline J0222.3-0009 & 022217.64 & 3.5 & -000934.37 & 2.1 & $S$ & $0.83 \pm 0.07$ & $<18.2$ & $<13.1$ & $55.0 \pm 35.9$ & -0.80 \\
\hline J0222.3-0242* & 022220.64 & 3.4 & -024220.89 & 2.1 & $S$ & $0.90 \pm 0.07$ & $<20.9$ & $<18.4$ & $175.2 \pm 60.2$ & -1.07 \\
\hline J0222.4+0059 & 022225.52 & 3.6 & +00 5925.45 & 2.3 & $S$ & $0.83 \pm 0.08$ & $<26.5$ & $<19.1$ & $150.6 \pm 29.9$ & -0.72 \\
\hline \multirow[t]{2}{*}{ J0222.5-0817 } & 022228.19 & 4.3 & -081708.81 & 4.0 & $M$ & $0.26 \pm 0.06$ & $<46.6$ & $<31.4$ & $9.0 \pm 34.7$ & -0.64 \\
\hline & 022229.57 & 3.8 & -08 1707.16 & 4.3 & $C$ & $0.16 \pm 0.04$ & $<51.4$ & $\ldots$ & $173.8 \pm 10.7$ & \\
\hline J0222.5-0307* & 022228.48 & 4.0 & -030742.30 & 3.7 & $S$ & $0.18 \pm 0.04$ & $<40.7$ & $<16.3$ & $7.9 \pm 20.8$ & -0.71 \\
\hline J0222.6-0614 & 022233.14 & 4.5 & -061446.89 & 4.4 & $S$ & $0.22 \pm 0.05$ & $<52.4$ & $<33.8$ & $7.9 \pm 31.2$ & -0.80 \\
\hline J0222.7+0059 & 022238.93 & 3.5 & +00 5920.03 & 2.3 & $S$ & $1.04 \pm 0.10$ & $<27.7$ & $<23.3$ & $171.7 \pm 45.3$ & -0.67 \\
\hline \multirow[t]{2}{*}{$\mathrm{J} 0222.8+0028$} & 022245.61 & 4.3 & +00 2824.97 & 3.0 & $M$ & $0.22 \pm 0.05$ & $<31.2$ & $<18.5$ & $73.5 \pm 39.9$ & -0.59 \\
\hline & 022245.58 & 3.7 & +002808.49 & 2.8 & $C$ & $0.21 \pm 0.04$ & $<26.4$ & & $26.2 \pm 19.1$ & \\
\hline J0222.9-0009 & 022251.19 & 4.1 & -000957.09 & 2.8 & $S$ & $0.45 \pm 0.07$ & $<42.1$ & $<23.6$ & $56.0 \pm 19.3$ & -0.64 \\
\hline J0222.9-0942 & 022253.18 & 4.7 & -094255.03 & 4.0 & $S$ & $0.37 \pm 0.08$ & $<51.5$ & $<33.8$ & $40.0 \pm 31.5$ & -0.82 \\
\hline J0222.9-0311* & 022253.94 & 4.1 & -031132.56 & 2.9 & $S$ & $0.20 \pm 0.04$ & $<34.3$ & $<17.5$ & $55.9 \pm 26.9$ & -1.10 \\
\hline J0222.9-0518* & 022255.86 & 3.4 & -051816.63 & 2.0 & $S$ & $1.56 \pm 0.09$ & $<26.2$ & $<21.2$ & $35.0 \pm 14.5$ & -0.60 \\
\hline \multirow{2}{*}{ J0223.0-0242* } & 022256.03 & 3.4 & -024304.05 & 2.0 & $M$ & $2.37 \pm 0.13$ & $<17.9$ & $<15.2$ & $71.0 \pm 22.0$ & -0.78 \\
\hline & 022258.38 & 3.4 & -024254.48 & 2.0 & $C$ & $1.87 \pm 0.11$ & $<24.2$ & $<17.2$ & $64.9 \pm 10.6$ & \\
\hline J0222.9-0602 & 022256.38 & 4.0 & -060206.79 & 3.1 & $S$ & $0.15 \pm 0.03$ & $<26.5$ & $<17.5$ & $10.6 \pm 57.1$ & -0.40 \\
\hline J0222.9-0424* & 022256.69 & 4.2 & -042450.37 & 3.2 & $S$ & $0.25 \pm 0.05$ & $<40.6$ & $<30.5$ & $39.5 \pm 41.8$ & -0.90 \\
\hline \multirow[t]{2}{*}{ J0223.0-0630 } & 022258.27 & 4.1 & -063052.20 & 3.0 & $M$ & $0.25 \pm 0.04$ & $<45.7$ & $<8.8$ & $45.3 \pm 11.2$ & -0.61 \\
\hline & 022258.46 & 5.1 & -063104.47 & 4.0 & $C$ & $0.22 \pm 0.05$ & $<47.3$ & $<38.9$ & $71.9 \pm 76.3$ & \\
\hline J0223.0-0606 & 022301.41 & 3.9 & -060626.01 & 3.5 & $S$ & $0.11 \pm 0.03$ & $<31.8$ & & $6.0 \pm 21.8$ & -0.67 \\
\hline J0223.0-0826 & 022301.93 & 4.5 & -082609.49 & 3.6 & $S$ & $0.68 \pm 0.10$ & $41.6 \pm 24.5$ & $<40.5$ & $45.9 \pm 15.8$ & -0.54 \\
\hline J0223.1-0107 & 022303.01 & 3.5 & -010746.46 & 2.2 & $S$ & $0.49 \pm 0.05$ & $<19.3$ & $<15.6$ & $107.0 \pm 66.2$ & -0.98 \\
\hline $\mathrm{J} 0223.2-0750$ & 022311.77 & 3.6 & -075031.67 & 2.3 & $S$ & $1.26 \pm 0.10$ & $35.6 \pm 11.5$ & $<32.2$ & $58.4 \pm 10.6$ & -0.65 \\
\hline J0223.2-0205* & 022312.89 & 3.7 & -020510.06 & 2.3 & $S$ & $0.29 \pm 0.04$ & $<22.6$ & $<5.6$ & $119.1 \pm 22.6$ & -0.26 \\
\hline J0223.3-0142 & 022318.67 & 4.5 & -014204.29 & 3.9 & $S$ & $0.21 \pm 0.05$ & $<41.0$ & $<32.3$ & $174.2 \pm 66.9$ & -0.70 \\
\hline J0223.3-1023 & 022320.80 & 4.7 & -102320.46 & 5.0 & $S$ & $0.66 \pm 0.14$ & $<67.6$ & $<42.6$ & $177.1 \pm 23.5$ & -0.99 \\
\hline J0223.4+0134 & 022326.37 & 4.7 & +013416.40 & 4.9 & $S$ & $0.54 \pm 0.12$ & $<67.9$ & $<34.1$ & $154.8 \pm 17.1$ & -0.81 \\
\hline J0223.5+0139 & 022332.28 & 5.0 & +013958.88 & 3.3 & $S$ & $0.25 \pm 0.06$ & $<43.1$ & $<22.3$ & $102.3 \pm 29.4$ & -0.72 \\
\hline J0223.6-0857 & 022332.99 & 5.6 & -085742.89 & 5.6 & $S$ & $0.41 \pm 0.10$ & $<68.7$ & $<53.9$ & $162.2 \pm 51.1$ & -0.53 \\
\hline J0223.6-0301* & 022333.10 & 3.5 & -030124.99 & 2.3 & $S$ & $0.47 \pm 0.05$ & $<22.2$ & $<19.2$ & $10.1 \pm 73.3$ & -0.82 \\
\hline J0223.6-0713 & 022333.22 & 4.0 & -071356.92 & 3.2 & $S$ & $0.81 \pm 0.10$ & $41.0 \pm 18.5$ & $<45.9$ & $21.1 \pm 22.8$ & -0.66 \\
\hline J0223.6-0232* & 022334.65 & 3.5 & -023218.36 & 2.2 & $S$ & $0.56 \pm 0.05$ & $<23.7$ & $<19.2$ & $55.8 \pm 44.9$ & -0.85 \\
\hline J0223.6-0637 & 022335.08 & 3.5 & -063722.32 & 2.2 & $S$ & $0.73 \pm 0.06$ & $<30.0$ & $<24.5$ & $51.2 \pm 32.0$ & -0.83 \\
\hline J0223.6-0213* & 022337.95 & 3.6 & -021355.29 & 2.5 & $S$ & $0.25 \pm 0.03$ & $<22.6$ & $<4.6$ & $37.4 \pm 23.7$ & -0.89 \\
\hline J0223.7-0239* & 022341.59 & 3.5 & -023944.16 & 2.2 & $S$ & $0.61 \pm 0.05$ & $<23.2$ & $<16.8$ & $54.2 \pm 28.1$ & -0.84 \\
\hline J0223.7-0158 & 022343.28 & 4.1 & -015835.77 & 3.4 & $S$ & $0.10 \pm 0.03$ & $<25.9$ & $<16.4$ & $176.0 \pm 67.2$ & -0.71 \\
\hline $\mathrm{J} 0223.7+0040$ & 022344.00 & 3.9 & +004048.97 & 2.7 & $S$ & $0.44 \pm 0.07$ & $<32.9$ & $<22.8$ & $125.5 \pm 34.1$ & -0.64 \\
\hline J0223.8-0340* & 022345.29 & 3.8 & -034042.32 & 2.9 & $S$ & $0.24 \pm 0.04$ & $<31.2$ & $<21.0$ & $9.8 \pm 35.9$ & -0.62 \\
\hline J0223.8-0332* & 022346.46 & 4.9 & -033237.04 & 3.7 & $S$ & $0.13 \pm 0.04$ & $<38.5$ & $<29.6$ & $114.9 \pm 73.6$ & -0.30 \\
\hline J0223.8-0412* & 022349.30 & 4.1 & -041219.81 & 3.4 & $S$ & $0.26 \pm 0.05$ & $<47.3$ & $<22.2$ & $146.9 \pm 16.7$ & -0.75 \\
\hline J0223.9+0233 & 022351.03 & 4.6 & +023319.48 & 4.2 & $S$ & $0.64 \pm 0.14$ & $<51.2$ & $<38.2$ & $166.8 \pm 42.2$ & -0.85 \\
\hline J0223.9+0012 & 022354.95 & 4.2 & +00 1235.18 & 3.6 & $S$ & $0.31 \pm 0.06$ & $<41.2$ & $<29.5$ & $165.8 \pm 40.9$ & -0.66 \\
\hline J0223.9-0619 & 022356.23 & 4.7 & -061931.60 & 3.2 & $S$ & $0.51 \pm 0.08$ & $<60.1$ & $<40.9$ & $75.0 \pm 21.8$ & -0.78 \\
\hline J0224.0-0441* & 022357.16 & 3.5 & -044111.62 & 2.2 & $S$ & $0.63 \pm 0.05$ & $<28.8$ & $<19.8$ & $19.1 \pm 18.7$ & -0.83 \\
\hline J0224.0-0638 & 022400.95 & 3.4 & -063844.07 & 2.0 & $S$ & $1.65 \pm 0.10$ & $22.9 \pm 9.3$ & $<21.4$ & $54.0 \pm 7.5$ & -0.87 \\
\hline J0224.1-0103 & 022404.55 & 3.5 & -010348.09 & 2.3 & $S$ & $0.41 \pm 0.04$ & $<16.8$ & $<13.0$ & $18.7 \pm 73.9$ & -0.57 \\
\hline J0224.2-0446* & 022409.91 & 4.7 & -044607.77 & 4.3 & $S$ & $0.15 \pm 0.04$ & $<43.6$ & $<32.7$ & $157.2 \pm 60.5$ & -0.67 \\
\hline J0224.2-0139 & 022412.61 & 4.0 & -013903.52 & 3.2 & $S$ & $0.26 \pm 0.05$ & $<36.1$ & $<26.5$ & $19.7 \pm 44.2$ & -0.50 \\
\hline J0224.2-0003 & 022412.63 & 3.5 & -000314.67 & 2.2 & $S$ & $0.61 \pm 0.06$ & $<22.4$ & $<11.2$ & $28.2 \pm 19.9$ & -0.92 \\
\hline \multirow{2}{*}{ J0224.2-0528* } & 022414.88 & 3.6 & -052839.39 & 2.3 & $M$ & $0.73 \pm 0.07$ & $<35.6$ & $<27.5$ & $152.2 \pm 22.8$ & -0.71 \\
\hline & 022413.04 & 3.7 & -052758.53 & 2.6 & $C$ & $0.69 \pm 0.07$ & $30.2 \pm 16.8$ & $<29.4$ & $147.8 \pm 13.0$ & \\
\hline J0224.3-0243* & 022417.86 & 4.7 & -024323.42 & 4.0 & $S$ & $0.37 \pm 0.07$ & $<64.3$ & $<35.6$ & $139.9 \pm 16.8$ & -0.39 \\
\hline J0224.3-0300* & 022418.97 & 4.4 & -030021.05 & 3.1 & $S$ & $0.19 \pm 0.04$ & $<40.3$ & $<23.3$ & $59.1 \pm 27.4$ & -0.70 \\
\hline J0224.4-0425* & 022421.13 & 4.5 & -042546.89 & 3.3 & $S$ & $0.29 \pm 0.05$ & $<47.3$ & $<35.7$ & $68.6 \pm 39.4$ & -0.89 \\
\hline J0224.4-0752 & 022422.46 & 4.2 & -075259.21 & 3.7 & $S$ & $0.45 \pm 0.07$ & $43.5 \pm 24.5$ & $<24.4$ & $33.8 \pm 8.4$ & -0.68 \\
\hline J0224.4-0129 & 022422.96 & 4.2 & -012932.86 & 3.5 & $S$ & $0.12 \pm 0.03$ & $<31.0$ & $<18.7$ & $166.8 \pm 48.5$ & -0.83 \\
\hline J0224.5-0243* & 022427.44 & 3.8 & -024308.68 & 3.1 & $S$ & $0.20 \pm 0.03$ & $<36.1$ & $<8.1$ & $22.8 \pm 15.9$ & -0.47 \\
\hline \multirow[t]{2}{*}{$\mathrm{J} 0224.5-0219 *$} & 022430.46 & 3.8 & -021937.14 & 2.6 & $M$ & $0.17 \pm 0.03$ & $<20.2$ & $<14.6$ & $120.5 \pm 80.5$ & -0.56 \\
\hline & 022430.53 & 5.0 & -021921.29 & 3.5 & $C$ & $0.13 \pm 0.03$ & $<52.2$ & $<4.3$ & $56.8 \pm 13.0$ & \\
\hline J0224.7-0209* & 022440.91 & 4.2 & -020959.04 & 3.8 & $S$ & $0.11 \pm 0.03$ & $<35.5$ & $<17.3$ & $175.7 \pm 34.1$ & -0.46 \\
\hline J0224.7-0732 & 022443.60 & 5.1 & -073234.35 & 4.0 & $S$ & $0.44 \pm 0.09$ & $<64.8$ & $<44.3$ & $56.1 \pm 26.0$ & -0.79 \\
\hline J0224.8-0302* & 022445.74 & 3.5 & -030205.78 & 2.3 & $M$ & $1.07 \pm 0.08$ & $35.0 \pm 10.7$ & $<24.0$ & $20.3 \pm 6.4$ & -0.76 \\
\hline & 022445.25 & 4.3 & -030252.69 & 2.8 & $C$ & $0.26 \pm 0.04$ & $<46.9$ & $<17.2$ & $61.3 \pm 12.9$ & \\
\hline & 022445.09 & 3.9 & -030239.26 & 2.5 & $C$ & $0.11 \pm 0.02$ & $<19.7$ & 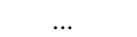 & $76.2 \pm 24.7$ & \\
\hline J0224.8-0545* & 022448.09 & 5.2 & -054527.33 & 4.1 & $S$ & $0.43 \pm 0.08$ & $49.1 \pm 30.0$ & $<40.4$ & $128.6 \pm 13.0$ & -0.77 \\
\hline J0224.8-0620 & 022448.67 & 4.2 & -062051.03 & 3.5 & $S$ & $0.19 \pm 0.04$ & $<37.4$ & $<26.0$ & $162.4 \pm 42.7$ & -0.63 \\
\hline
\end{tabular}


Table A.2. continued.

\begin{tabular}{|c|c|c|c|c|c|c|c|c|c|c|}
\hline Name & $\begin{array}{c}\text { RA } \\
\mathrm{J} 2000 \\
\end{array}$ & $\begin{array}{c}\sigma_{\alpha} \\
\operatorname{arcsec}\end{array}$ & $\begin{array}{c}\text { Dec } \\
\mathrm{J} 2000 \\
\end{array}$ & $\begin{array}{c}\sigma_{\delta} \\
\operatorname{arcsec}\end{array}$ & Type & $\begin{array}{c}S_{\text {comp }} \\
\text { Jy }\end{array}$ & $\begin{array}{c}\text { Major axis } \\
\text { arcsec }\end{array}$ & $\begin{array}{c}\text { Minor axis } \\
\text { arcsec }\end{array}$ & $\begin{array}{c}\text { Position } \\
\text { Angle } \\
\end{array}$ & $\begin{array}{c}\text { Spectral } \\
\text { index } \alpha_{74}^{1400}\end{array}$ \\
\hline $\mathrm{J} 0224.8-0221^{*}$ & 022448.97 & 3.9 & -022115.58 & 3.2 & $S$ & $0.27 \pm 0.04$ & $<45.0$ & $<21.6$ & $26.7 \pm 16.0$ & -0.64 \\
\hline J0224.9-0712 & 022456.78 & 5.1 & -071245.13 & 5.3 & $S$ & $0.33 \pm 0.08$ & $<67.3$ & $<46.1$ & $163.4 \pm 32.1$ & -0.90 \\
\hline J0225.0-0516* & 022457.36 & 4.2 & -051655.98 & 3.2 & $S$ & $0.32 \pm 0.05$ & $<50.0$ & $<28.1$ & $44.2 \pm 17.7$ & -0.74 \\
\hline J0225.0-0942 & 022500.13 & 4.4 & -094236.14 & 3.7 & $S$ & $0.86 \pm 0.13$ & $42.0 \pm 24.7$ & $<36.5$ & $39.1 \pm 13.2$ & -0.48 \\
\hline J0225.0-0321* & 022500.22 & 5.0 & -032106.56 & 5.0 & $S$ & $0.20 \pm 0.05$ & $<57.7$ & $<40.1$ & $162.3 \pm 39.9$ & -0.92 \\
\hline J0225.0+0131 & 022501.39 & 3.7 & +013136.96 & 2.5 & $S$ & $1.01 \pm 0.11$ & $<36.1$ & $<26.9$ & $140.7 \pm 25.1$ & -0.88 \\
\hline J0225.1-0536* & 022505.18 & 3.4 & -053646.36 & 2.0 & $S$ & $1.46 \pm 0.09$ & $<28.2$ & $<24.2$ & $27.7 \pm 22.2$ & -0.61 \\
\hline J0225.1-0828 & 022505.93 & 4.9 & -082803.25 & 5.1 & $S$ & $0.27 \pm 0.07$ & $<67.0$ & $<31.1$ & $29.4 \pm 17.7$ & -0.67 \\
\hline J0225.1+0017 & 022507.74 & 3.4 & +00 1704.82 & 2.0 & $S$ & $1.98 \pm 0.12$ & $<22.6$ & $<17.2$ & $23.4 \pm 17.8$ & -0.66 \\
\hline J0225.1-0035 & 022507.77 & 3.4 & -003533.22 & 2.0 & $S$ & $7.78 \pm 0.39$ & $12.1 \pm 10.1$ & $<13.6$ & $62.9 \pm 2.7$ & -0.64 \\
\hline J0225.1-0938 & 022508.11 & 6.6 & -093830.09 & 4.2 & $S$ & $0.68 \pm 0.15$ & $48.4 \pm 38.5$ & $<47.7$ & $77.8 \pm 16.8$ & -0.81 \\
\hline \multirow[t]{2}{*}{ J0225.2-0738 } & 022508.79 & 3.7 & -073836.19 & 2.3 & $M$ & $1.45 \pm 0.12$ & $42.8 \pm 12.0$ & $22.6 \pm 15.4$ & $75.4 \pm 10.7$ & -0.69 \\
\hline & 022509.96 & 3.7 & -073912.86 & 2.3 & $C$ & $1.01 \pm 0.09$ & $35.8 \pm 13.8$ & $<29.1$ & $67.7 \pm 9.6$ & \\
\hline J0225.3-0930 & 022515.86 & 4.0 & -093058.58 & 3.2 & $S$ & $1.01 \pm 0.13$ & $37.2 \pm 20.7$ & $<38.4$ & $31.4 \pm 16.8$ & -0.76 \\
\hline J0225.4-0056 & 022525.27 & 4.1 & -005638.82 & 3.3 & $S$ & $0.19 \pm 0.04$ & $<29.8$ & $<20.5$ & $153.6 \pm 56.8$ & -0.84 \\
\hline J0225.4-0248* & 022525.96 & 4.6 & -024815.02 & 3.9 & $S$ & $0.16 \pm 0.04$ & $<38.5$ & $\ldots$ & $\ldots$ & -0.70 \\
\hline J0225.6-0210* & 022534.05 & 3.4 & -021018.07 & 2.1 & $S$ & $0.63 \pm 0.05$ & $<23.1$ & $<13.9$ & $55.0 \pm 16.9$ & -0.12 \\
\hline J0225.8-0637 & 022545.10 & 5.1 & -063750.79 & 4.8 & $S$ & $0.24 \pm 0.06$ & $<55.2$ & $<45.3$ & $4.8 \pm 69.1$ & -0.76 \\
\hline \multirow[t]{2}{*}{ J0225.9-0545* } & 022554.74 & 4.2 & -054543.23 & 5.1 & $M$ & $0.13 \pm 0.03$ & $<63.1$ & $<5.5$ & $162.9 \pm 10.7$ & -0.46 \\
\hline & 022555.58 & 3.9 & -054540.32 & 3.2 & $C$ & $0.10 \pm 0.02$ & $<29.1$ & & $18.6 \pm 24.6$ & \\
\hline J0225.9+0219 & 022555.51 & 3.6 & +02 1914.30 & 2.8 & $S$ & $0.57 \pm 0.08$ & $<34.2$ & $<3.4$ & $7.6 \pm 12.6$ & -0.57 \\
\hline J0225.9+0151 & 022555.56 & 4.7 & +015129.22 & 3.5 & $S$ & $0.41 \pm 0.09$ & $<42.7$ & $<34.1$ & $100.9 \pm 62.8$ & -0.74 \\
\hline J0226.2-0133 & 022609.14 & 4.5 & -013353.20 & 4.7 & $S$ & $0.16 \pm 0.04$ & $<59.1$ & $<22.2$ & $24.5 \pm 16.2$ & -0.53 \\
\hline J0226.2+0129 & 022609.18 & 3.4 & +012926.14 & 2.1 & $S$ & $1.80 \pm 0.12$ & $<28.4$ & $<17.3$ & $3.0 \pm 10.8$ & -0.54 \\
\hline J0226.2-0646 & 022614.12 & 5.6 & -064600.60 & 4.4 & $S$ & $0.18 \pm 0.05$ & $<52.2$ & $<40.0$ & $68.7 \pm 60.1$ & -0.74 \\
\hline $\mathrm{J} 0226.2+0205$ & 022614.83 & 3.7 & +020511.31 & 2.4 & $S$ & $1.49 \pm 0.14$ & $36.6 \pm 13.9$ & $<22.5$ & $130.2 \pm 6.9$ & -0.49 \\
\hline J0226.3-0400* & 022618.81 & 4.8 & -040010.04 & 4.4 & $S$ & $0.30 \pm 0.06$ & $<57.2$ & $<46.7$ & $12.6 \pm 54.2$ & -0.41 \\
\hline J0226.3-0425* & 022619.88 & 3.7 & -042536.25 & 2.6 & $S$ & $0.34 \pm 0.04$ & $<29.3$ & $<20.4$ & $13.5 \pm 31.9$ & -0.73 \\
\hline J0226.4-0846 & 022625.36 & 3.6 & -084630.78 & 2.2 & $S$ & $2.01 \pm 0.15$ & $39.2 \pm 10.3$ & $24.0 \pm 12.6$ & $53.8 \pm 11.2$ & -0.63 \\
\hline J0226.6-0806 & 022634.57 & 3.6 & -080618.67 & 2.3 & $S$ & $1.23 \pm 0.10$ & $35.1 \pm 12.2$ & $<29.5$ & $44.6 \pm 9.4$ & -0.68 \\
\hline J0226.6-0715 & 022635.98 & 3.7 & -071500.53 & 2.5 & $S$ & $0.60 \pm 0.06$ & $<41.8$ & $<22.3$ & $43.2 \pm 11.9$ & -0.87 \\
\hline J0226.7-0121 & 022639.33 & 3.6 & -012124.44 & 2.4 & $S$ & $0.44 \pm 0.05$ & $<30.0$ & $<18.7$ & $44.6 \pm 21.6$ & -0.86 \\
\hline J0226.7-0552* & 022640.23 & 4.1 & -055237.19 & 3.4 & $S$ & $0.24 \pm 0.04$ & $<43.3$ & $<24.3$ & $23.3 \pm 22.8$ & -0.26 \\
\hline J0226.8-0712 & 022645.18 & 3.6 & -071212.89 & 2.3 & $S$ & $0.92 \pm 0.08$ & $29.2 \pm 13.7$ & $<27.3$ & $39.9 \pm 11.3$ & -0.85 \\
\hline J0227.0-0223* & 022657.08 & 4.5 & -022354.07 & 3.8 & $S$ & $0.24 \pm 0.05$ & $<56.0$ & $<27.2$ & $140.9 \pm 17.0$ & -0.89 \\
\hline J0227.0+0206 & 022659.06 & 3.7 & +020611.95 & 2.8 & $S$ & $0.87 \pm 0.11$ & $<38.2$ & $<24.9$ & $176.8 \pm 21.6$ & -0.68 \\
\hline J0227.0-0555* & 022659.31 & 3.4 & -055544.34 & 2.0 & $S$ & $2.46 \pm 0.13$ & $13.5 \pm 11.7$ & $<22.5$ & $37.2 \pm 18.6$ & -0.76 \\
\hline J0227.0+0224 & 022700.39 & 4.5 & +02 2419.73 & 4.2 & $S$ & $0.37 \pm 0.09$ & $<49.1$ & $<23.1$ & $152.8 \pm 22.4$ & -0.58 \\
\hline J0227.2-0325* & 022708.52 & 4.6 & -032556.57 & 4.0 & $S$ & $0.36 \pm 0.07$ & $<56.7$ & $<44.3$ & $31.6 \pm 39.9$ & -0.52 \\
\hline J0227.2-0141 & 022711.56 & 5.5 & -014151.25 & 3.6 & $S$ & $0.23 \pm 0.05$ & $<61.0$ & $<32.7$ & $75.0 \pm 20.9$ & -1.07 \\
\hline J0227.2-0053 & 022712.29 & 3.4 & -005344.98 & 2.1 & $S$ & $0.86 \pm 0.06$ & $<19.0$ & $<12.0$ & $49.6 \pm 21.2$ & -1.06 \\
\hline \multirow[t]{2}{*}{ J0227.4-0240* } & 022722.85 & 4.8 & -023956.17 & 3.7 & $M$ & $0.24 \pm 0.05$ & $<46.5$ & $<39.3$ & $93.2 \pm 75.9$ & -0.79 \\
\hline & 022725.85 & 4.6 & -024017.96 & 3.2 & $C$ & $0.18 \pm 0.04$ & $<39.9$ & $<27.3$ & $87.4 \pm 41.6$ & \\
\hline J0227.4+0137 & 022726.57 & 3.5 & +013733.32 & 2.4 & $S$ & $0.96 \pm 0.09$ & $<34.7$ & $<16.0$ & $5.3 \pm 10.9$ & -0.74 \\
\hline J0227.5-0437* & 022728.11 & 4.7 & -043731.87 & 4.1 & $S$ & $0.20 \pm 0.05$ & $<50.2$ & $<35.2$ & $146.1 \pm 37.7$ & -0.74 \\
\hline J0227.5-0557* & 022731.82 & 6.4 & -055745.09 & 3.1 & $S$ & $0.35 \pm 0.07$ & $54.2 \pm 33.6$ & $<27.6$ & $93.7 \pm 8.4$ & -0.58 \\
\hline J0227.6-0314* & 022735.72 & 4.6 & -031445.55 & 4.2 & $S$ & $0.12 \pm 0.03$ & $<47.8$ & $<20.5$ & $33.7 \pm 23.6$ & -0.65 \\
\hline J0227.6-0052 & 022736.25 & 3.7 & -005258.18 & 2.5 & $S$ & $0.56 \pm 0.06$ & $<31.7$ & & & -0.70 \\
\hline J0227.6+0133 & 022737.11 & 3.7 & +013312.08 & 2.9 & $S$ & $0.55 \pm 0.08$ & $<36.1$ & $<18.6$ & $1.7 \pm 18.6$ & -0.65 \\
\hline J0227.7-0922 & 022740.09 & 5.7 & -092219.76 & 4.3 & $S$ & $0.44 \pm 0.11$ & $<65.3$ & $<39.9$ & $60.1 \pm 25.4$ & -0.51 \\
\hline J0227.7-0908 & 022740.47 & 4.4 & -090848.91 & 3.2 & $S$ & $1.27 \pm 0.16$ & $48.0 \pm 19.8$ & $<49.4$ & $56.9 \pm 19.0$ & -0.83 \\
\hline J0227.7-0043 & 022741.27 & 4.1 & -004345.66 & 2.6 & $S$ & $0.28 \pm 0.05$ & $<34.8$ & $<16.8$ & $80.9 \pm 21.1$ & -0.63 \\
\hline J0227.7-0250* & 022744.22 & 3.5 & -025048.77 & 2.3 & $S$ & $0.35 \pm 0.04$ & $<25.6$ & & $148.5 \pm 12.5$ & -0.68 \\
\hline J0227.7-0621 & 022744.63 & 4.2 & -062107.22 & 3.3 & $S$ & $0.27 \pm 0.05$ & $<41.0$ & $<32.3$ & $34.0 \pm 49.9$ & 0.23 \\
\hline J0227.8+0221 & 022745.17 & 3.7 & +022142.43 & 3.0 & $S$ & $1.03 \pm 0.13$ & $<46.2$ & $<21.2$ & $172.9 \pm 11.6$ & -0.89 \\
\hline J0227.9-0035 & 022753.72 & 3.7 & -003535.32 & 2.7 & $S$ & $0.24 \pm 0.04$ & $<26.0$ & $<14.6$ & $12.4 \pm 34.4$ & -0.38 \\
\hline \multirow[t]{2}{*}{ J0228.0-0644 } & 022802.20 & 3.6 & -064440.12 & 2.5 & $M$ & $0.22 \pm 0.03$ & $<23.4$ & $\ldots$ & $174.8 \pm 18.9$ & -0.58 \\
\hline & 022803.40 & 3.9 & -064425.34 & 5.6 & $C$ & $0.18 \pm 0.04$ & $<74.2$ & & $5.9 \pm 7.7$ & \\
\hline $\mathrm{J} 0228.1+0031$ & 022805.61 & 3.4 & +00 3117.25 & 2.0 & $S$ & $1.59 \pm 0.10$ & $<22.0$ & $<19.7$ & $15.0 \pm 45.4$ & -0.61 \\
\hline J0228.1-0231* & 022807.48 & 3.4 & -023107.26 & 2.0 & $S$ & $1.81 \pm 0.10$ & $<18.6$ & $<17.2$ & $75.2 \pm 45.8$ & -0.91 \\
\hline J0228.1-0115 & 022807.54 & 3.4 & -011543.86 & 2.0 & $S$ & $4.88 \pm 0.25$ & $20.8 \pm 6.7$ & $<15.6$ & $94.1 \pm 2.7$ & -0.80 \\
\hline J0228.2-0046 & 022810.43 & 3.5 & -004601.11 & 2.2 & $S$ & $0.75 \pm 0.07$ & $<26.2$ & $<19.2$ & $129.4 \pm 25.8$ & -0.87 \\
\hline J0228.3-0157 & 022819.99 & 3.7 & -015755.33 & 2.6 & $S$ & $0.23 \pm 0.03$ & $<23.6$ & $<13.6$ & $175.7 \pm 37.4$ & -0.54 \\
\hline $\mathrm{J} 0228.4+0032$ & 022825.03 & 4.0 & +00 3210.99 & 3.8 & $S$ & $0.39 \pm 0.07$ & $<52.1$ & $<24.6$ & $11.1 \pm 15.8$ & -1.06 \\
\hline J0228.5-0223* & 022829.16 & 3.5 & -022317.89 & 2.3 & $S$ & $0.55 \pm 0.05$ & $<23.1$ & $\ldots$ & $\ldots$ & -0.79 \\
\hline J0228.6+0150 & 022833.64 & 4.6 & +015036.91 & 3.7 & $S$ & $0.45 \pm 0.10$ & $<39.9$ & & . & -0.69 \\
\hline J0228.7-0602 & 022840.58 & 3.4 & -060242.61 & 2.1 & $S$ & $1.07 \pm 0.08$ & $<27.6$ & $<22.3$ & $38.9 \pm 23.1$ & -0.79 \\
\hline J0228.7-0042 & 022840.95 & 4.8 & -004255.24 & 3.9 & $S$ & $0.21 \pm 0.05$ & $<43.9$ & $<35.5$ & $51.6 \pm 71.2$ & -0.70 \\
\hline J0228.8-0617 & 022848.10 & 3.4 & -061753.62 & 2.0 & $S$ & $1.80 \pm 0.11$ & $<28.4$ & $<27.0$ & $46.7 \pm 58.7$ & -1.00 \\
\hline \multirow[t]{2}{*}{$\mathrm{J} 0228.8+0049$} & 022849.04 & 3.8 & +004946.91 & 2.7 & $M$ & $0.31 \pm 0.05$ & $<30.5$ & $<7.9$ & $41.5 \pm 18.0$ & -0.78 \\
\hline & 022848.92 & 4.3 & +005001.60 & 3.3 & $C$ & $0.14 \pm 0.03$ & $<43.3$ & & $46.2 \pm 10.4$ & \\
\hline J0228.9-0337* & 022853.17 & 3.4 & -033737.75 & 2.0 & $S$ & $6.07 \pm 0.31$ & $<18.5$ & $<16.4$ & $62.1 \pm 12.0$ & -0.63 \\
\hline J0229.0+0052 & 022858.53 & 4.6 & +005255.83 & 5.0 & $S$ & $0.28 \pm 0.07$ & $<56.5$ & $<30.6$ & $177.7 \pm 25.2$ & -0.60 \\
\hline J0229.1-0655 & 022904.22 & 4.9 & -065522.27 & 3.6 & $S$ & $0.39 \pm 0.07$ & $<64.3$ & $<29.7$ & $54.5 \pm 13.4$ & -0.81 \\
\hline
\end{tabular}


Table A.2. continued.

\begin{tabular}{|c|c|c|c|c|c|c|c|c|c|c|}
\hline Name & $\begin{array}{c}\text { RA } \\
\text { J2000 } \\
\end{array}$ & $\begin{array}{c}\sigma_{\alpha} \\
\operatorname{arcsec}\end{array}$ & $\begin{array}{c}\text { Dec } \\
\mathrm{J} 2000 \\
\end{array}$ & $\begin{array}{c}\sigma_{\delta} \\
\operatorname{arcsec}\end{array}$ & Type & $\begin{array}{c}S_{\text {comp }} \\
\mathrm{Jy}\end{array}$ & $\begin{array}{c}\text { Major axis } \\
\text { arcsec }\end{array}$ & $\begin{array}{c}\text { Minor axis } \\
\text { arcsec }\end{array}$ & $\begin{array}{c}\text { Position } \\
\text { Angle }\end{array}$ & $\begin{array}{c}\text { Spectral } \\
\text { index } \alpha_{74}^{1400}\end{array}$ \\
\hline J0229.1-0541* & 022905.73 & 3.4 & -054110.76 & 2.1 & $S$ & $1.17 \pm 0.08$ & $19.1 \pm 13.2$ & $<24.5$ & $31.4 \pm 14.6$ & -0.57 \\
\hline J0229.1-0223* & 022908.76 & 3.7 & -022308.01 & 2.7 & $S$ & $0.21 \pm 0.03$ & $<29.1$ & $<10.1$ & $26.4 \pm 20.8$ & -0.66 \\
\hline J0229.2-0656 & 022910.13 & 3.7 & -065604.58 & 2.4 & $S$ & $0.79 \pm 0.08$ & $<40.2$ & $<27.7$ & $56.7 \pm 16.6$ & -0.81 \\
\hline J0229.2-0311* & 022911.92 & 4.7 & -031135.17 & 4.1 & $S$ & $0.17 \pm 0.04$ & $<52.2$ & $<20.8$ & $140.6 \pm 19.1$ & -0.65 \\
\hline J0229.3-0442* & 022915.90 & 3.4 & -044214.91 & 2.0 & $S$ & $2.97 \pm 0.16$ & $<23.9$ & $<18.2$ & $45.0 \pm 7.9$ & -0.72 \\
\hline \multirow[t]{3}{*}{ J0229.6-0027 } & 022932.48 & 4.5 & -002740.63 & 3.9 & $M$ & $0.43 \pm 0.08$ & $<64.2$ & $<28.7$ & $37.7 \pm 12.6$ & -0.76 \\
\hline & 022933.70 & 5.1 & -002644.68 & 3.8 & $C$ & $0.33 \pm 0.07$ & $<51.5$ & $<41.1$ & $79.4 \pm 55.8$ & \\
\hline & 022933.87 & 4.0 & -002625.98 & 3.0 & $C$ & $0.32 \pm 0.05$ & $<43.4$ & $<15.3$ & $44.6 \pm 13.7$ & \\
\hline J0229.6-0752 & 022937.56 & 4.6 & -075230.20 & 3.8 & $S$ & $0.34 \pm 0.07$ & $<55.8$ & $<28.0$ & $43.4 \pm 17.9$ & -0.59 \\
\hline \multirow[t]{2}{*}{ J0229.6+0025 } & 022938.67 & 3.5 & +002526.05 & 2.2 & $M$ & $1.13 \pm 0.09$ & $22.8 \pm 15.0$ & $<15.2$ & $47.8 \pm 7.3$ & -0.79 \\
\hline & 022936.88 & 3.4 & +00 2459.95 & 2.1 & $C$ & $1.11 \pm 0.08$ & $<22.5$ & $<16.3$ & $148.8 \pm 24.2$ & \\
\hline J0229.7-0238* & 022938.91 & 4.0 & -023837.57 & 3.1 & $S$ & $0.16 \pm 0.03$ & $<37.5$ & $<12.7$ & $141.1 \pm 18.9$ & -0.35 \\
\hline J0229.7-0705 & 022943.13 & 3.4 & -070537.64 & 2.0 & $S$ & $2.29 \pm 0.14$ & $25.6 \pm 8.6$ & $15.0 \pm 13.2$ & $90.0 \pm 13.5$ & -0.90 \\
\hline J0229.7-0148 & 022944.20 & 5.6 & -014824.63 & 4.5 & $S$ & $0.22 \pm 0.06$ & $<52.4$ & & & -1.02 \\
\hline J0229.8-0206 & 022947.29 & 3.5 & -020648.36 & 2.3 & $S$ & $0.64 \pm 0.05$ & $<33.1$ & $<21.0$ & $176.4 \pm 14.0$ & -0.84 \\
\hline J0230.0-0331* & 023001.18 & 3.7 & -033151.27 & 2.8 & $S$ & $0.20 \pm 0.03$ & $<28.5$ & $<10.4$ & $15.1 \pm 23.1$ & -0.67 \\
\hline J0230.1-0209 & 023003.64 & 4.0 & -020922.60 & 3.3 & $S$ & $0.10 \pm 0.02$ & $<30.1$ & $<9.6$ & $27.7 \pm 29.9$ & -0.41 \\
\hline J0230.1-0628 & 023005.16 & 3.8 & -062803.68 & 2.8 & $S$ & $0.47 \pm 0.06$ & $<38.7$ & $<31.8$ & $18.2 \pm 44.0$ & -0.84 \\
\hline J0230.2-0642 & 023010.88 & 3.5 & -064227.99 & 2.3 & $S$ & $0.73 \pm 0.07$ & $<30.7$ & $<23.4$ & $32.2 \pm 24.8$ & -1.11 \\
\hline J0230.2-0056 & 023014.12 & 3.8 & -005658.04 & 2.7 & $S$ & $0.23 \pm 0.04$ & $<27.2$ & $<20.8$ & $18.5 \pm 58.0$ & -0.79 \\
\hline J0230.3-1007 & 023017.48 & 4.8 & -100708.05 & 5.3 & $S$ & $0.94 \pm 0.19$ & $43.0 \pm 34.8$ & $<49.9$ & $7.2 \pm 21.3$ & -0.68 \\
\hline J0230.3-0750 & 023017.77 & 3.8 & -075035.26 & 2.6 & $S$ & $0.91 \pm 0.10$ & $33.5 \pm 16.6$ & $<30.7$ & $54.6 \pm 12.2$ & -0.69 \\
\hline J0230.4-0255* & 023021.92 & 3.4 & -025505.60 & 2.0 & $S$ & $2.08 \pm 0.11$ & $<18.6$ & $<16.6$ & $15.1 \pm 30.3$ & -1.07 \\
\hline J0230.4-0412* & 023023.51 & 3.4 & -041227.64 & 2.1 & $S$ & $0.65 \pm 0.05$ & $<17.9$ & $<15.5$ & $0.5 \pm 73.0$ & -0.37 \\
\hline J0230.4-0830 & 023026.11 & 3.6 & -083040.61 & 2.2 & $S$ & $2.12 \pm 0.16$ & $42.0 \pm 10.4$ & $<32.2$ & $58.6 \pm 7.2$ & -0.74 \\
\hline J0230.4+0108 & 023026.34 & 4.2 & +010848.17 & 3.8 & $S$ & $0.77 \pm 0.12$ & $41.4 \pm 24.4$ & $<34.9$ & $155.2 \pm 12.6$ & -0.60 \\
\hline J0230.6-0309* & 023033.26 & 3.7 & -030909.60 & 2.7 & $S$ & $0.32 \pm 0.04$ & $<31.2$ & $<20.9$ & $16.9 \pm 29.9$ & -0.62 \\
\hline J0230.6-0421* & 023033.41 & 3.9 & -042107.50 & 3.0 & $S$ & $0.18 \pm 0.03$ & $<33.6$ & $<10.3$ & $28.8 \pm 19.9$ & -0.29 \\
\hline J0230.6-0638 & 023034.89 & 4.1 & -063846.51 & 3.6 & $S$ & $0.29 \pm 0.05$ & $<48.0$ & $<25.8$ & $157.9 \pm 19.7$ & -0.69 \\
\hline J0230.6-0414 & 023036.04 & 4.5 & -041448.60 & 4.6 & $S$ & $0.15 \pm 0.04$ & $<48.0$ & $<26.9$ & $173.8 \pm 30.9$ & -0.77 \\
\hline J0230.6-0409 & 023036.88 & 3.5 & -040935.16 & 2.2 & $S$ & $0.57 \pm 0.05$ & $<23.1$ & $<16.8$ & $35.3 \pm 29.4$ & -0.61 \\
\hline J0230.7+0020 & 023039.75 & 4.6 & +00 2019.15 & 3.9 & $S$ & $0.49 \pm 0.09$ & $<64.7$ & $<29.6$ & $140.0 \pm 13.0$ & -0.94 \\
\hline $\mathrm{J} 0230.7-0328^{*}$ & 023040.92 & 3.5 & -032838.77 & 2.4 & $S$ & $0.37 \pm 0.04$ & $<25.6$ & $<13.7$ & $6.1 \pm 21.7$ & -0.60 \\
\hline J0230.7-0256* & 023043.30 & 5.4 & -025622.48 & 6.0 & $S$ & $0.42 \pm 0.09$ & $47.8 \pm 40.2$ & $<51.0$ & $25.4 \pm 18.7$ & -0.60 \\
\hline J0230.7-0701 & 023043.59 & 3.6 & -070119.47 & 2.3 & $S$ & $0.79 \pm 0.07$ & $<36.1$ & $<23.5$ & $44.9 \pm 15.0$ & -0.82 \\
\hline J0230.7-0840 & 023044.11 & 5.1 & -084044.05 & 3.9 & $S$ & $0.62 \pm 0.12$ & $<59.7$ & $<47.2$ & $65.4 \pm 43.3$ & -0.82 \\
\hline J0230.8-0336 & 023049.06 & 4.4 & -033615.57 & 4.1 & $S$ & $0.12 \pm 0.03$ & $<38.2$ & $<25.9$ & $173.5 \pm 52.1$ & -0.74 \\
\hline J0230.8-0721 & 023049.99 & 4.4 & -072100.52 & 3.5 & $S$ & $0.24 \pm 0.05$ & $<37.3$ & $<29.5$ & $142.5 \pm 66.2$ & -0.45 \\
\hline J0230.9+0014 & 023053.42 & 4.2 & +001416.50 & 3.7 & $S$ & $0.34 \pm 0.07$ & $<45.4$ & $<26.4$ & $22.1 \pm 25.7$ & -0.71 \\
\hline J0230.9-0352 & 023053.62 & 5.3 & -035259.98 & 4.2 & $S$ & $0.19 \pm 0.05$ & $<56.0$ & $<32.3$ & $53.4 \pm 27.5$ & -0.73 \\
\hline J0230.9-0909 & 023055.18 & 3.8 & -090952.55 & 2.5 & $S$ & $1.85 \pm 0.17$ & $39.7 \pm 13.7$ & $27.9 \pm 15.3$ & $63.6 \pm 21.1$ & -0.74 \\
\hline J0231.0-0451* & 023057.55 & 4.2 & -045138.93 & 3.1 & $S$ & $0.24 \pm 0.04$ & $<34.8$ & & $\ldots$ & -0.56 \\
\hline J0231.0-0624 & 023058.25 & 3.7 & -062447.55 & 2.6 & $S$ & $0.46 \pm 0.06$ & $<30.4$ & $<25.7$ & $168.7 \pm 57.8$ & -0.68 \\
\hline J0231.0+0149 & 023100.50 & 3.4 & +014958.15 & 2.1 & $S$ & $2.93 \pm 0.19$ & $24.1 \pm 10.4$ & $<24.1$ & $165.9 \pm 9.9$ & -1.08 \\
\hline J0231.0-0049 & 023100.85 & 3.5 & -004944.98 & 2.2 & $S$ & $0.55 \pm 0.05$ & $<22.0$ & $<16.5$ & $61.4 \pm 37.3$ & -1.12 \\
\hline J0231.0-0014 & 023102.59 & 3.4 & -001403.68 & 2.0 & $S$ & $2.16 \pm 0.13$ & $32.3 \pm 7.7$ & $<19.4$ & $141.6 \pm 4.0$ & -0.95 \\
\hline J0231.1-0822 & 023105.39 & 6.9 & -082242.65 & 3.6 & $S$ & $1.68 \pm 0.24$ & $105.0 \pm 31.5$ & $30.1 \pm 24.5$ & $103.5 \pm 5.9$ & -1.18 \\
\hline J0231.3-0019 & 023116.94 & 4.6 & -001956.01 & 3.5 & $S$ & $0.19 \pm 0.05$ & $<39.0$ & $<26.5$ & $60.0 \pm 45.6$ & -0.75 \\
\hline J0231.4-0315 & 023126.53 & 4.1 & -031501.18 & 3.2 & $S$ & $0.29 \pm 0.05$ & $<36.6$ & & & -0.78 \\
\hline J0231.4-0421 & 023126.69 & 3.4 & -042134.58 & 2.0 & $S$ & $1.84 \pm 0.10$ & $<22.9$ & $<20.1$ & $47.7 \pm 25.2$ & -0.59 \\
\hline J0231.5-0212 & 023130.25 & 3.7 & -021244.10 & 2.8 & $S$ & $0.21 \pm 0.03$ & $<27.2$ & $<11.8$ & $168.8 \pm 27.1$ & -0.69 \\
\hline J0231.5-0228 & 023131.88 & 3.5 & -022803.96 & 2.1 & $S$ & $0.62 \pm 0.05$ & $<20.6$ & $<18.5$ & $56.7 \pm 80.7$ & -0.60 \\
\hline J0231.5-0041 & 023132.37 & 4.2 & -004148.12 & 3.6 & $S$ & $0.25 \pm 0.05$ & $<41.5$ & $<27.4$ & $15.4 \pm 34.3$ & -0.70 \\
\hline \multirow[t]{2}{*}{ J0231.7-0453 } & 023140.39 & 3.5 & -045256.48 & 2.1 & $M$ & $1.80 \pm 0.12$ & $32.7 \pm 9.1$ & $22.6 \pm 11.2$ & $81.1 \pm 13.8$ & -0.76 \\
\hline & 023137.16 & 3.6 & -045316.92 & 2.4 & $C$ & $0.73 \pm 0.07$ & $<34.1$ & $<26.3$ & $14.9 \pm 24.9$ & \\
\hline J0231.8-0326 & 023145.81 & 6.0 & -032615.69 & 4.4 & $S$ & $0.25 \pm 0.06$ & $<62.2$ & $<45.2$ & $83.4 \pm 42.4$ & -1.20 \\
\hline J0231.8-0305 & 023150.49 & 4.1 & -030559.15 & 3.9 & $S$ & $0.18 \pm 0.04$ & $<44.7$ & $<21.2$ & $167.1 \pm 21.6$ & -0.60 \\
\hline \multirow{2}{*}{ J0231.9-0226 } & 023152.25 & 3.5 & -022646.71 & 2.4 & $M$ & $0.38 \pm 0.04$ & $<26.5$ & $<9.0$ & $173.5 \pm 15.5$ & -0.82 \\
\hline & 023151.20 & 4.0 & -022653.37 & 3.0 & $C$ & $0.30 \pm 0.05$ & $<39.2$ & $<23.0$ & $37.1 \pm 22.7$ & \\
\hline J0232.1-0627 & 023208.66 & 5.4 & -062725.45 & 3.8 & $S$ & $0.45 \pm 0.09$ & $<66.9$ & $<39.2$ & $68.0 \pm 20.0$ & -0.52 \\
\hline $\mathrm{J} 0232.2+0022$ & 023209.46 & 4.0 & +002235.46 & 3.3 & $S$ & $0.32 \pm 0.06$ & $<38.3$ & $<22.6$ & $25.2 \pm 28.0$ & -0.80 \\
\hline J0232.2-0441 & 023214.14 & 3.4 & -044150.62 & 2.1 & $S$ & $1.31 \pm 0.08$ & $<29.2$ & $<18.2$ & $21.6 \pm 9.3$ & -0.75 \\
\hline J0232.3-0749 & 023215.65 & 3.8 & -074948.89 & 2.6 & $S$ & $0.85 \pm 0.10$ & $<39.7$ & $<33.6$ & $61.6 \pm 40.7$ & -0.73 \\
\hline J0232.3-0033 & 023215.81 & 4.2 & -003303.33 & 3.9 & $S$ & $0.28 \pm 0.06$ & $<49.5$ & $<24.1$ & $162.5 \pm 19.2$ & -0.48 \\
\hline J0232.3-0545 & 023216.64 & 4.1 & -054519.63 & 3.6 & $S$ & $0.19 \pm 0.04$ & $<42.2$ & $<13.9$ & $27.5 \pm 17.8$ & -0.56 \\
\hline J0232.3-0401 & 023217.64 & 5.5 & -040132.56 & 5.0 & $S$ & $0.34 \pm 0.07$ & $48.6 \pm 38.1$ & $<28.7$ & $42.6 \pm 9.7$ & -0.65 \\
\hline J0232.4-0525 & 023221.97 & 4.2 & -052547.17 & 3.8 & $S$ & $0.17 \pm 0.04$ & $<40.7$ & $<21.7$ & $160.3 \pm 28.5$ & -0.65 \\
\hline J0232.4-0003 & 023226.74 & 5.1 & -000322.92 & 4.7 & $S$ & $0.34 \pm 0.08$ & $<71.3$ & $<27.4$ & $39.8 \pm 13.3$ & -0.74 \\
\hline $\mathrm{J} 0232.5+0113$ & 023228.77 & 3.9 & +011354.28 & 3.3 & $S$ & $0.31 \pm 0.06$ & $<34.8$ & $<15.6$ & $5.6 \pm 23.8$ & -0.65 \\
\hline J0232.5-0418 & 023232.87 & 3.8 & -041831.04 & 2.7 & $S$ & $0.40 \pm 0.06$ & $<33.8$ & $<26.6$ & $39.3 \pm 43.6$ & -0.51 \\
\hline J0232.6-0428 & 023234.27 & 4.3 & -042834.17 & 3.2 & $S$ & $0.29 \pm 0.05$ & $<45.2$ & $<26.0$ & $47.4 \pm 21.9$ & -0.75 \\
\hline J0232.6-0104 & 023237.09 & 5.5 & -010404.35 & 4.6 & $S$ & $0.26 \pm 0.07$ & $<52.3$ & & 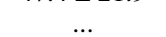 & -0.44 \\
\hline J0232.6-0444 & 023237.84 & 4.3 & -044442.51 & 3.8 & $S$ & $0.25 \pm 0.05$ & $<47.7$ & $<30.2$ & $19.9 \pm 27.6$ & -0.60 \\
\hline J0232.6-0105 & 023238.83 & 4.3 & -010519.83 & 3.5 & $S$ & $0.54 \pm 0.09$ & $<49.4$ & $<43.8$ & $22.0 \pm 73.6$ & -0.59 \\
\hline
\end{tabular}


Table A.2. continued.

\begin{tabular}{|c|c|c|c|c|c|c|c|c|c|c|}
\hline Name & $\begin{array}{c}\text { RA } \\
\text { J2000 }\end{array}$ & $\begin{array}{c}\sigma_{\alpha} \\
\operatorname{arcsec}\end{array}$ & $\begin{array}{c}\text { Dec } \\
\text { J2000 }\end{array}$ & $\begin{array}{c}\sigma_{\delta} \\
\operatorname{arcsec}\end{array}$ & Type & $\begin{array}{c}S_{\text {comp }} \\
\text { Jy }\end{array}$ & $\begin{array}{c}\text { Major axis } \\
\text { arcsec }\end{array}$ & $\begin{array}{c}\text { Minor axis } \\
\text { arcsec }\end{array}$ & $\begin{array}{c}\text { Position } \\
\text { Angle }\end{array}$ & $\begin{array}{c}\text { Spectral } \\
\text { index } \alpha_{74}^{1400}\end{array}$ \\
\hline $\mathrm{J} 0232.6+0056$ & 023238.86 & 3.7 & +005620.00 & 2.4 & $S$ & $1.20 \pm 0.12$ & $<37.4$ & $<33.3$ & $101.4 \pm 51.7$ & -1.04 \\
\hline \multirow[t]{4}{*}{$\mathrm{J} 0232.7-0233$} & 023241.87 & 3.5 & -023309.22 & 2.2 & $M$ & $2.28 \pm 0.15$ & $40.0 \pm 8.8$ & $37.9 \pm 8.9$ & $51.8 \pm 60.5$ & -0.72 \\
\hline & 023245.81 & 3.4 & -023429.22 & 2.2 & $C$ & $2.03 \pm 0.13$ & $41.8 \pm 8.4$ & $<29.4$ & $159.4 \pm 5.2$ & \\
\hline & 023243.24 & 4.8 & -023354.99 & 4.0 & C & $0.41 \pm 0.07$ & $43.1 \pm 30.3$ & $<26.9$ & $45.4 \pm 9.7$ & \\
\hline & 023245.09 & 5.4 & -023353.18 & 4.7 & $C$ & $0.23 \pm 0.05$ & $54.5 \pm 35.6$ & & $44.3 \pm 4.6$ & \\
\hline J0232.7-0632 & 023244.80 & 4.1 & -063209.47 & 3.0 & $S$ & $0.32 \pm 0.06$ & $<31.9$ & $<24.8$ & $126.5 \pm 59.5$ & -0.52 \\
\hline J0232.8-0829 & 023244.97 & 5.1 & -083000.14 & 4.7 & $S$ & $0.40 \pm 0.10$ & $<60.6$ & $<40.2$ & $35.0 \pm 31.9$ & -0.75 \\
\hline \multirow[t]{2}{*}{$\mathrm{J} 0232.8-0425$} & 023249.47 & 4.2 & -042531.20 & 3.1 & $M$ & $0.24 \pm 0.04$ & $<47.6$ & $<13.8$ & $132.2 \pm 12.5$ & -0.57 \\
\hline & 023249.96 & 4.4 & -042534.52 & 3.6 & C & $0.23 \pm 0.04$ & $<58.0$ & $<9.4$ & $41.0 \pm 9.5$ & \\
\hline $\mathrm{J} 0232.8+0017$ & 023250.10 & 3.5 & +001720.30 & 2.2 & $S$ & $0.78 \pm 0.07$ & $<25.1$ & $<14.5$ & $179.9 \pm 18.7$ & -0.54 \\
\hline $\mathrm{J} 0232.9+0002$ & 023251.91 & 4.9 & +000235.01 & 4.4 & $S$ & $0.36 \pm 0.09$ & $<50.9$ & $<43.6$ & $7.8 \pm 85.8$ & -0.70 \\
\hline J0232.9-0645 & 023254.06 & 3.4 & -064552.37 & 2.0 & $S$ & $10.38 \pm 0.53$ & $17.6 \pm 7.1$ & $14.3 \pm 8.6$ & $68.7 \pm 12.0$ & -0.67 \\
\hline J0232.9-0351 & 023255.82 & 4.0 & -035141.05 & 3.0 & $S$ & $0.21 \pm 0.04$ & $<30.6$ & $<19.2$ & $37.3 \pm 38.1$ & -0.51 \\
\hline J0233.1-0843 & 023303.92 & 4.5 & -084336.66 & 3.1 & $S$ & $1.03 \pm 0.14$ & $44.6 \pm 21.9$ & $<36.5$ & $57.8 \pm 11.9$ & -0.98 \\
\hline J0233.2-0220 & 023310.36 & 4.1 & -022001.01 & 3.3 & $S$ & $0.20 \pm 0.04$ & $<33.8$ & $<21.7$ & $24.8 \pm 39.7$ & -0.43 \\
\hline J0233.2-0012 & 023313.39 & 3.8 & -001218.51 & 2.1 & $S$ & $2.23 \pm 0.16$ & $72.9 \pm 10.7$ & $<21.7$ & $78.7 \pm 2.2$ & -1.00 \\
\hline \multirow{2}{*}{ J0233.3-0320 } & 023318.88 & 3.4 & -032055.05 & 2.0 & $M$ & $5.33 \pm 0.28$ & $45.2 \pm 4.5$ & $<20.3$ & $86.4 \pm 1.3$ & -0.75 \\
\hline & 023315.79 & 3.5 & -032055.74 & 2.2 & $C$ & $1.06 \pm 0.08$ & $<31.2$ & $<25.3$ & $177.6 \pm 24.9$ & \\
\hline J0233.3-0455 & 023320.24 & 3.9 & -045501.14 & 3.1 & $S$ & $0.44 \pm 0.07$ & $<44.2$ & $<31.1$ & $21.6 \pm 26.2$ & -0.25 \\
\hline J0233.4-0454 & 023324.38 & 3.7 & -045500.22 & 2.4 & $S$ & $0.62 \pm 0.07$ & $<34.6$ & $<27.1$ & $68.4 \pm 29.9$ & -0.37 \\
\hline J0233.4+0015 & 023325.43 & 4.5 & +001547.92 & 3.2 & $S$ & $0.56 \pm 0.10$ & $<49.9$ & $<36.5$ & $93.8 \pm 31.6$ & -0.83 \\
\hline J0233.4-0435 & 023326.02 & 3.9 & -043555.87 & 3.0 & $S$ & $0.38 \pm 0.06$ & $<39.2$ & $<25.6$ & $22.4 \pm 25.1$ & -1.06 \\
\hline \multirow[t]{3}{*}{ J0233.5-0713 } & 023328.69 & 4.1 & -071324.63 & 2.9 & $M$ & $0.83 \pm 0.11$ & $40.4 \pm 19.9$ & $<27.5$ & $128.7 \pm 9.2$ & -1.03 \\
\hline & 023329.90 & 4.4 & -071319.86 & 4.0 & $C$ & $0.39 \pm 0.08$ & $<58.6$ & $<23.6$ & $34.6 \pm 13.6$ & \\
\hline & 023327.30 & 4.5 & -071242.42 & 3.7 & C & $0.29 \pm 0.06$ & $<47.6$ & $<23.3$ & $42.1 \pm 21.4$ & \\
\hline J0233.5-0203 & 023330.22 & 3.4 & -020322.92 & 2.0 & $S$ & $2.23 \pm 0.13$ & $<21.6$ & $<17.0$ & $45.6 \pm 15.2$ & -0.66 \\
\hline J0233.6-0412 & 023333.60 & 4.0 & -041238.78 & 3.1 & $S$ & $0.18 \pm 0.04$ & $<31.6$ & $<16.7$ & $28.5 \pm 32.4$ & -0.35 \\
\hline J0233.6-0519 & 023333.86 & 4.1 & -051953.28 & 3.3 & $S$ & $0.33 \pm 0.06$ & $<40.7$ & $<30.8$ & $25.5 \pm 41.9$ & -0.77 \\
\hline J0233.6-0306 & 023336.28 & 4.0 & -030637.27 & 3.4 & $S$ & $0.16 \pm 0.04$ & $<30.5$ & $<16.4$ & $11.6 \pm 38.8$ & -0.53 \\
\hline J0233.6-0059 & 023337.50 & 3.7 & -010000.24 & 2.9 & $S$ & $0.18 \pm 0.03$ & $<27.1$ & $\ldots$ & $174.2 \pm 21.3$ & -0.62 \\
\hline J0233.7-0639 & 023339.54 & 4.5 & -063935.25 & 3.6 & $S$ & $0.20 \pm 0.05$ & $<34.2$ & $\ldots$ & $\ldots$ & -0.55 \\
\hline \multirow[t]{2}{*}{ J0233.7-0912 } & 023341.50 & 4.1 & -091245.55 & 5.3 & $M$ & $0.65 \pm 0.12$ & $57.5 \pm 30.9$ & $<13.3$ & $18.3 \pm 5.7$ & -0.97 \\
\hline & 023340.25 & 4.5 & -091241.13 & 3.9 & $C$ & $0.58 \pm 0.12$ & $<50.2$ & $<37.6$ & $24.0 \pm 40.1$ & \\
\hline J0233.7-0232 & 023343.88 & 3.4 & -023211.08 & 2.0 & $S$ & $2.06 \pm 0.12$ & $26.6 \pm 8.1$ & $<20.2$ & $64.0 \pm 5.2$ & -0.93 \\
\hline J0233.8-0052 & 023346.62 & 4.4 & -005235.54 & 3.4 & $S$ & $0.30 \pm 0.06$ & $<42.4$ & $<32.2$ & $137.6 \pm 45.5$ & -0.62 \\
\hline \multirow{2}{*}{ J0234.0-0508 } & 023357.16 & 3.4 & -050759.93 & 2.4 & $M$ & $2.08 \pm 0.14$ & $56.4 \pm 9.1$ & $<26.2$ & $173.3 \pm 3.1$ & -1.08 \\
\hline & 023358.07 & 3.7 & -050854.58 & 2.8 & $C$ & $0.80 \pm 0.09$ & $34.0 \pm 17.5$ & $<30.7$ & $157.7 \pm 12.2$ & \\
\hline $\mathrm{J} 0234.0+0150$ & 023401.23 & 3.9 & +015011.29 & 3.0 & $S$ & $0.98 \pm 0.14$ & $<46.2$ & $<27.6$ & $155.2 \pm 17.3$ & -0.73 \\
\hline $\mathrm{J} 0234.1+0055$ & 023403.05 & 3.7 & +00 5538.17 & 2.7 & $S$ & $0.34 \pm 0.05$ & $<24.3$ & $<11.9$ & $3.6 \pm 33.9$ & -0.86 \\
\hline J0234.2-0308 & 023410.89 & 4.1 & -030827.98 & 3.9 & $S$ & $0.21 \pm 0.04$ & $<45.8$ & $<18.6$ & $16.9 \pm 18.5$ & -0.76 \\
\hline J0234.2-0121 & 023410.90 & 4.1 & -012144.68 & 2.8 & $S$ & $0.37 \pm 0.06$ & $<37.4$ & $<28.6$ & $102.2 \pm 40.8$ & -0.78 \\
\hline J0234.2-0025 & 023411.71 & 4.0 & -002554.03 & 2.8 & $S$ & $0.39 \pm 0.06$ & $<33.9$ & $<26.5$ & $86.1 \pm 50.7$ & -0.79 \\
\hline J0234.2-0603 & 023412.17 & 3.5 & -060358.85 & 2.2 & $S$ & $0.82 \pm 0.07$ & $<28.2$ & $<24.6$ & $5.1 \pm 49.5$ & -0.71 \\
\hline $\mathrm{J} 0234.3+0123$ & 023415.61 & 3.7 & +012340.39 & 3.0 & $S$ & $0.68 \pm 0.09$ & $<41.2$ & $<20.5$ & $172.7 \pm 15.2$ & -0.96 \\
\hline J0234.3-0935 & 023416.36 & 3.9 & -093522.11 & 3.2 & $S$ & $2.09 \pm 0.23$ & $47.6 \pm 16.9$ & $<44.3$ & $167.4 \pm 14.1$ & -0.41 \\
\hline J0234.3+0149 & 023417.04 & 4.0 & +014932.36 & 4.6 & $S$ & $0.29 \pm 0.07$ & $<52.0$ & $<8.3$ & $177.8 \pm 13.8$ & -0.74 \\
\hline J0234.3-0415 & 023420.70 & 4.0 & -041544.82 & 3.6 & $S$ & $0.26 \pm 0.05$ & $<42.4$ & $<23.0$ & $7.3 \pm 24.1$ & -0.70 \\
\hline J0234.4-0523 & 023421.55 & 3.5 & -052333.64 & 2.3 & $S$ & $0.61 \pm 0.06$ & $<27.4$ & $<16.1$ & $5.1 \pm 17.2$ & -0.87 \\
\hline J0234.4-0139 & 023421.68 & 3.5 & -013901.00 & 2.2 & $S$ & $0.51 \pm 0.05$ & $<20.0$ & $<13.7$ & $166.4 \pm 35.7$ & -0.65 \\
\hline J0234.4-0645 & 023423.74 & 3.5 & -064537.20 & 2.3 & $M$ & $2.15 \pm 0.16$ & $50.5 \pm 10.1$ & $<30.6$ & $147.0 \pm 4.8$ & -0.90 \\
\hline & 023422.64 & 4.0 & -064504.42 & 2.7 & $C$ & $0.30 \pm 0.05$ & $<34.2$ & $<14.6$ & $60.9 \pm 20.8$ & \\
\hline J0234.4-0013 & 023426.86 & 3.4 & -001342.68 & 2.1 & $S$ & $1.32 \pm 0.09$ & $<21.7$ & $<20.1$ & $25.4 \pm 79.7$ & -0.69 \\
\hline J0234.5-0150 & 023427.40 & 4.4 & -015013.15 & 4.2 & $S$ & $0.21 \pm 0.05$ & $<52.8$ & $<21.2$ & $150.3 \pm 17.6$ & -0.92 \\
\hline J0234.5-0245 & 023431.25 & 4.9 & -024519.60 & 5.1 & $S$ & $0.25 \pm 0.06$ & $<62.6$ & $<36.8$ & $19.1 \pm 25.9$ & -0.91 \\
\hline J0234.6-0419 & 023435.89 & 4.8 & -041933.17 & 5.2 & $S$ & $0.39 \pm 0.09$ & $<76.5$ & $<31.5$ & $154.3 \pm 12.8$ & -0.56 \\
\hline J0234.8-0050 & 023445.53 & 3.4 & -005024.09 & 2.1 & $S$ & $1.25 \pm 0.09$ & $<25.1$ & $<21.6$ & $101.7 \pm 34.8$ & -0.69 \\
\hline J0235.0-0736 & 023458.75 & 3.7 & -073618.52 & 2.4 & $S$ & $0.89 \pm 0.09$ & $<35.0$ & $<28.7$ & $112.3 \pm 34.3$ & -0.75 \\
\hline J0235.0-0115 & 023501.03 & 4.7 & -011510.04 & 3.2 & $S$ & $0.28 \pm 0.06$ & $<47.7$ & $<26.8$ & $77.0 \pm 23.3$ & -0.70 \\
\hline J0235.0-0532 & 023501.57 & 4.5 & -053235.25 & 4.6 & $S$ & $0.17 \pm 0.04$ & $<54.2$ & $<22.9$ & $157.8 \pm 19.6$ & -0.76 \\
\hline J0235.1-0402 & 023507.02 & 3.4 & -040203.39 & 2.0 & $S$ & $10.80 \pm 0.54$ & $17.3 \pm 6.9$ & $<16.3$ & $74.1 \pm 2.2$ & -0.66 \\
\hline $\mathrm{J} 0235.2+0033$ & 023510.30 & 3.6 & +003338.17 & 2.6 & $S$ & $0.52 \pm 0.07$ & $<29.1$ & $<15.7$ & $8.1 \pm 22.3$ & -0.50 \\
\hline J0235.2-0159 & 023512.77 & 3.8 & -015957.26 & 2.9 & $S$ & $0.30 \pm 0.05$ & $<36.2$ & $<14.6$ & $31.4 \pm 17.0$ & -0.53 \\
\hline J0235.3-0704 & 023516.78 & 4.0 & -070419.57 & 3.2 & $S$ & $0.33 \pm 0.06$ & $<37.4$ & $<26.5$ & $14.1 \pm 38.1$ & -0.59 \\
\hline J0235.3-0012 & 023518.29 & 4.4 & -001251.12 & 3.2 & $S$ & $0.34 \pm 0.06$ & $<41.0$ & $<28.8$ & $112.1 \pm 37.3$ & -0.70 \\
\hline J0235.4-0654 & 023521.44 & 4.3 & -065454.48 & 5.3 & $S$ & $0.40 \pm 0.08$ & $<74.7$ & $<31.1$ & $171.0 \pm 12.5$ & -0.55 \\
\hline J0235.4+0133 & 023524.11 & 5.1 & +013349.98 & 5.1 & $S$ & $0.59 \pm 0.14$ & $<61.0$ & $<46.9$ & $4.1 \pm 47.9$ & -1.02 \\
\hline J0235.5-0037 & 023528.23 & 4.1 & -003709.09 & 3.8 & $S$ & $0.15 \pm 0.04$ & $<37.7$ & $<15.2$ & $16.3 \pm 26.1$ & -0.61 \\
\hline J0235.5-0705 & 023530.79 & 4.3 & -070502.62 & 3.5 & $S$ & $0.35 \pm 0.07$ & $<43.6$ & $<32.9$ & $150.1 \pm 43.3$ & -0.87 \\
\hline J0235.5-0219 & 023532.46 & 3.4 & -021932.00 & 2.0 & $S$ & $5.80 \pm 0.30$ & $<21.9$ & $<17.3$ & $42.8 \pm 6.5$ & -0.60 \\
\hline J0235.6-0515 & 023535.03 & 4.3 & -051558.41 & 4.1 & $S$ & $0.23 \pm 0.05$ & $<49.9$ & $<23.6$ & $158.1 \pm 20.1$ & -0.54 \\
\hline J0235.6-0557 & 023537.01 & 3.8 & -055702.19 & 2.9 & $S$ & $0.38 \pm 0.05$ & $<38.9$ & $<23.9$ & $163.4 \pm 22.2$ & -0.76 \\
\hline J0235.7-0759 & 023542.21 & 4.1 & -075959.26 & 2.9 & $S$ & $0.62 \pm 0.09$ & $<42.6$ & $<31.9$ & $57.9 \pm 33.1$ & -0.52 \\
\hline J0235.8-0524 & 023544.90 & 4.2 & -052436.88 & 3.5 & $S$ & $0.20 \pm 0.04$ & $<37.4$ & $<26.1$ & $163.1 \pm 44.0$ & -0.41 \\
\hline J0235.9-0803 & 023554.50 & 4.2 & -080349.68 & 3.6 & $M$ & $0.68 \pm 0.10$ & $42.8 \pm 24.3$ & $<20.0$ & $143.0 \pm 7.6$ & -0.63 \\
\hline
\end{tabular}


Table A.2. continued.

\begin{tabular}{|c|c|c|c|c|c|c|c|c|c|c|}
\hline Name & $\begin{array}{c}\text { RA } \\
\text { J2000 }\end{array}$ & $\begin{array}{c}\sigma_{\alpha} \\
\operatorname{arcsec}\end{array}$ & $\begin{array}{c}\text { Dec } \\
\text { J2000 }\end{array}$ & $\begin{array}{c}\sigma_{\delta} \\
\operatorname{arcsec}\end{array}$ & Type & $\begin{array}{c}S_{\text {comp }} \\
\text { Jy }\end{array}$ & $\begin{array}{c}\text { Major axis } \\
\text { arcsec }\end{array}$ & $\begin{array}{c}\text { Minor axis } \\
\text { arcsec }\end{array}$ & $\begin{array}{c}\text { Position } \\
\text { Angle }\end{array}$ & $\begin{array}{c}\text { Spectral } \\
\text { index } \alpha_{74}^{1400}\end{array}$ \\
\hline & 023555.67 & 4.2 & -080337.17 & 3.1 & $\bar{C}$ & $0.48 \pm 0.08$ & $<42.1$ & $<28.0$ & $46.9 \pm 28.8$ & \\
\hline J0236.1-0250 & 023606.38 & 3.5 & -025010.74 & 2.2 & $S$ & $0.84 \pm 0.07$ & $<28.0$ & $<18.4$ & $24.0 \pm 17.0$ & -0.90 \\
\hline J0236.1-0628 & 023608.59 & 4.6 & -062828.02 & 4.0 & $S$ & $0.34 \pm 0.07$ & $<59.3$ & $<30.9$ & $140.8 \pm 17.7$ & -0.61 \\
\hline J0236.3-0157 & 023615.45 & 3.9 & -015732.86 & 2.8 & $S$ & $0.31 \pm 0.05$ & $<31.1$ & $<23.9$ & $46.0 \pm 50.7$ & -0.63 \\
\hline J0236.4-0437 & 023621.62 & 3.7 & -043738.72 & 2.4 & $S$ & $0.76 \pm 0.08$ & $<35.9$ & $<30.2$ & $91.8 \pm 37.1$ & -0.87 \\
\hline J0236.4-0730 & 023624.99 & 6.3 & -073029.42 & 4.8 & $S$ & $0.42 \pm 0.10$ & $<81.2$ & $<41.5$ & $122.9 \pm 17.7$ & -0.91 \\
\hline J0236.4-0334 & 023626.30 & 4.5 & -033450.75 & 3.7 & $S$ & $0.28 \pm 0.06$ & $<51.6$ & $<25.9$ & $139.7 \pm 19.1$ & -0.47 \\
\hline J0236.6-0358 & 023637.72 & 3.9 & -035824.54 & 3.0 & $S$ & $0.13 \pm 0.03$ & $<22.5$ & $<15.3$ & $177.5 \pm 75.4$ & -0.62 \\
\hline $\mathrm{J} 0236.7+0131$ & 023639.65 & 5.0 & +013110.79 & 4.7 & $S$ & $0.42 \pm 0.11$ & $<60.3$ & $<32.3$ & $145.2 \pm 22.8$ & -0.90 \\
\hline J0236.8-0301 & 023645.81 & 3.8 & -030153.48 & 3.1 & $S$ & $0.22 \pm 0.04$ & $<39.4$ & $\ldots$ & $28.7 \pm 12.6$ & -0.61 \\
\hline J0236.9-0231 & 023651.78 & 4.1 & -023111.72 & 4.2 & $S$ & $0.26 \pm 0.05$ & $<55.3$ & $<25.2$ & $170.2 \pm 16.2$ & -0.46 \\
\hline J0236.9-0428 & 023652.45 & 4.6 & -042853.92 & 3.3 & $S$ & $0.16 \pm 0.04$ & $<38.9$ & $<18.4$ & $60.3 \pm 27.5$ & -0.47 \\
\hline J0237.2-0348 & 023710.40 & 3.4 & -034824.23 & 2.0 & $S$ & $2.06 \pm 0.11$ & $<24.2$ & $<17.1$ & $62.3 \pm 9.0$ & -0.79 \\
\hline J0237.6-0245 & 023736.63 & 4.6 & -024505.46 & 3.9 & $S$ & $0.14 \pm 0.04$ & $<38.7$ & $<26.7$ & $33.5 \pm 53.6$ & -0.58 \\
\hline $\mathrm{J} 0237.8-0055$ & 023746.36 & 4.2 & -005528.69 & 3.5 & $S$ & $0.41 \pm 0.07$ & $<47.1$ & $<31.9$ & $151.2 \pm 28.1$ & -0.78 \\
\hline J0237.8-0719 & 023746.91 & 5.4 & -071907.97 & 5.2 & $S$ & $0.30 \pm 0.07$ & $<82.8$ & $<24.5$ & $141.2 \pm 10.1$ & -1.02 \\
\hline J0238.0-0145 & 023757.05 & 3.4 & -014511.66 & 2.0 & $S$ & $4.05 \pm 0.21$ & $<24.0$ & $<20.2$ & $58.1 \pm 11.3$ & -0.79 \\
\hline J0238.2-0628 & 023814.24 & 3.5 & -062851.58 & 2.2 & $S$ & $1.14 \pm 0.09$ & $<34.4$ & $<24.8$ & $104.7 \pm 15.5$ & -0.59 \\
\hline J0238.3-0257 & 023815.37 & 4.4 & -025744.71 & 3.8 & $S$ & $0.21 \pm 0.05$ & $<40.4$ & $<28.8$ & $16.9 \pm 47.2$ & -0.61 \\
\hline J0238.3-0330 & 023818.77 & 3.4 & -033018.42 & 2.0 & $S$ & $2.98 \pm 0.16$ & $<22.4$ & $<18.9$ & $77.1 \pm 13.4$ & -0.72 \\
\hline J0238.4-0616 & 023823.66 & 3.5 & -061618.26 & 2.2 & $S$ & $1.13 \pm 0.09$ & $<27.9$ & $<26.0$ & $148.9 \pm 78.3$ & -0.59 \\
\hline J0238.4-0348 & 023824.15 & 4.3 & -034829.57 & 3.6 & $S$ & $0.27 \pm 0.05$ & $<42.9$ & $<32.8$ & $5.0 \pm 47.4$ & -0.56 \\
\hline J0238.5-0616 & 023828.24 & 3.5 & -061616.96 & 2.3 & $S$ & $0.96 \pm 0.08$ & $<32.5$ & $<23.8$ & $168.4 \pm 19.1$ & -0.79 \\
\hline $\mathrm{J} 0238.5-0425$ & 023828.39 & 4.1 & -042536.56 & 3.9 & $S$ & $0.38 \pm 0.07$ & $<62.5$ & $<24.9$ & $25.0 \pm 10.8$ & -0.62 \\
\hline J0238.6-0607 & 023832.97 & 4.5 & -060727.66 & 4.0 & $S$ & $0.19 \pm 0.05$ & $<41.0$ & $<28.0$ & $19.1 \pm 46.6$ & -0.56 \\
\hline J0238.6-0432 & 023835.24 & 3.5 & -043237.91 & 2.2 & $S$ & $0.73 \pm 0.06$ & $<30.1$ & $<20.4$ & $95.3 \pm 17.2$ & -0.65 \\
\hline J0238.6-0103 & 023837.56 & 3.9 & -010314.25 & 2.9 & $S$ & $0.38 \pm 0.06$ & $<32.8$ & $<27.6$ & $164.0 \pm 70.2$ & -0.77 \\
\hline J0238.9-0156 & 023853.86 & 4.3 & -015607.70 & 3.3 & $S$ & $0.35 \pm 0.06$ & $<44.1$ & $<32.3$ & $50.3 \pm 37.2$ & -0.69 \\
\hline J0239.0-0255 & 023857.05 & 3.5 & -025508.62 & 2.1 & $S$ & $0.81 \pm 0.06$ & $<24.6$ & $<20.7$ & $66.6 \pm 38.6$ & -0.61 \\
\hline $\mathrm{J} 0239.0+0056$ & 023859.94 & 3.8 & +005612.33 & 3.0 & $S$ & $0.60 \pm 0.09$ & $<38.0$ & $<20.5$ & $155.7 \pm 20.3$ & -0.69 \\
\hline J0239.2-0851 & 023909.75 & 4.9 & -085112.85 & 4.9 & $S$ & $0.70 \pm 0.15$ & $<68.7$ & $<46.5$ & $161.5 \pm 26.9$ & -0.99 \\
\hline J0239.2-0715 & 023912.88 & 6.5 & -071546.40 & 5.4 & $S$ & $0.65 \pm 0.15$ & $50.0 \pm 41.2$ & $<57.6$ & $126.4 \pm 22.3$ & -0.96 \\
\hline J0239.2-0118 & 023913.47 & 3.4 & -011815.78 & 2.0 & $S$ & $3.18 \pm 0.18$ & $21.1 \pm 8.6$ & $<19.0$ & $51.1 \pm 5.9$ & -0.87 \\
\hline J0239.4-0413 & 023925.59 & 4.3 & -041301.46 & 3.3 & $S$ & $0.15 \pm 0.03$ & $<32.0$ & $<22.3$ & $45.0 \pm 54.4$ & -0.40 \\
\hline J0239.6-0506 & 023933.01 & 4.2 & -050613.85 & 3.1 & $S$ & $0.16 \pm 0.03$ & $<28.4$ & $<17.9$ & $128.5 \pm 51.1$ & -0.51 \\
\hline J0239.6-0845 & 023937.66 & 4.6 & -084531.77 & 4.0 & $S$ & $1.17 \pm 0.18$ & $48.6 \pm 25.2$ & $<44.5$ & $37.6 \pm 14.9$ & -0.76 \\
\hline J0239.7-0218 & 023939.79 & 3.8 & -021839.05 & 3.0 & $S$ & $0.25 \pm 0.04$ & $<33.4$ & $<18.1$ & $2.1 \pm 25.7$ & -0.49 \\
\hline J0239.7-0117 & 023942.42 & 3.5 & -011717.38 & 2.1 & $S$ & $1.19 \pm 0.09$ & $<26.4$ & $<20.8$ & $52.1 \pm 25.2$ & -0.83 \\
\hline J0239.8-0234 & 023945.73 & 5.1 & -023440.34 & 4.4 & $S$ & $0.31 \pm 0.07$ & $<67.0$ & $<32.0$ & $136.5 \pm 16.5$ & 0.00 \\
\hline J0239.8-0057 & 023948.88 & 5.1 & -005740.73 & 3.7 & $S$ & $0.45 \pm 0.09$ & $<58.1$ & $<37.6$ & $63.1 \pm 26.0$ & -0.74 \\
\hline J0239.9-0302 & 023952.97 & 4.2 & -030206.54 & 3.2 & $S$ & $0.14 \pm 0.03$ & $<26.5$ & $<20.1$ & $135.1 \pm 86.3$ & -0.54 \\
\hline J0240.0-0057 & 023957.07 & 4.1 & -005751.76 & 3.3 & $S$ & $0.52 \pm 0.09$ & $<42.7$ & $<35.3$ & $3.4 \pm 52.8$ & -0.67 \\
\hline J0240.1-0636 & 024006.39 & 4.1 & -063642.22 & 4.2 & $S$ & $0.37 \pm 0.07$ & $<60.6$ & $<24.5$ & $161.4 \pm 12.6$ & -0.60 \\
\hline J0240.2-0331 & 024011.38 & 4.9 & -033119.20 & 4.2 & $S$ & $0.38 \pm 0.08$ & $<68.7$ & $<32.9$ & $139.1 \pm 14.2$ & -0.70 \\
\hline J0240.3-0331 & 024016.23 & 5.9 & -033139.64 & 3.9 & $S$ & $0.40 \pm 0.09$ & $<71.9$ & $<42.8$ & $101.4 \pm 20.8$ & -0.58 \\
\hline J0240.4-0130 & 024025.80 & 3.6 & -013025.62 & 2.3 & $S$ & $0.65 \pm 0.07$ & $<29.6$ & $<18.4$ & $57.5 \pm 20.0$ & -0.78 \\
\hline J0240.5-0128 & 024030.24 & 4.9 & -012814.53 & 3.4 & $S$ & $0.27 \pm 0.06$ & $<50.8$ & $<22.2$ & $116.3 \pm 18.7$ & -0.66 \\
\hline J0240.6-0408 & 024035.30 & 4.1 & -040851.53 & 3.4 & $S$ & $0.65 \pm 0.09$ & $49.5 \pm 20.7$ & $<26.9$ & $144.5 \pm 7.3$ & -0.88 \\
\hline \multirow[t]{2}{*}{ J0240.6-0723 } & 024036.70 & 4.0 & -072317.07 & 2.9 & $M$ & $0.69 \pm 0.09$ & $37.1 \pm 20.3$ & $<20.2$ & $134.5 \pm 7.9$ & -0.56 \\
\hline & 024037.81 & 5.5 & -072307.96 & 3.5 & $C$ & $0.37 \pm 0.08$ & $<63.1$ & $<30.4$ & $71.4 \pm 16.9$ & \\
\hline J0240.7-0638 & 024039.53 & 3.8 & -063825.09 & 2.7 & $S$ & $0.88 \pm 0.10$ & $31.3 \pm 18.4$ & $<23.1$ & $140.7 \pm 9.4$ & -0.59 \\
\hline $\mathrm{J} 0240.7-0446$ & 024041.16 & 3.5 & -044614.92 & 2.1 & $S$ & $1.06 \pm 0.08$ & $<29.0$ & $<19.3$ & $63.7 \pm 13.8$ & -0.62 \\
\hline \multirow[t]{2}{*}{ J0240.9-0138 } & 024053.58 & 3.7 & -013753.59 & 3.5 & $M$ & $0.91 \pm 0.11$ & $53.9 \pm 18.6$ & $<22.8$ & $171.8 \pm 5.5$ & -0.69 \\
\hline & 024054.50 & 3.8 & -013824.58 & 2.8 & $C$ & $0.40 \pm 0.06$ & $<33.1$ & $<21.5$ & $20.5 \pm 29.7$ & \\
\hline J0240.9-0504 & 024056.51 & 4.9 & -050441.35 & 4.4 & $S$ & $0.37 \pm 0.08$ & $<54.5$ & $<46.5$ & $178.6 \pm 75.3$ & -0.15 \\
\hline J0241.0-0022 & 024057.21 & 3.7 & -002251.46 & 2.7 & $S$ & $0.63 \pm 0.09$ & $<31.2$ & $<22.8$ & $8.3 \pm 36.4$ & -0.68 \\
\hline J0241.0-0020 & 024058.66 & 4.1 & -002031.66 & 3.2 & $S$ & $0.50 \pm 0.09$ & $<35.2$ & $\ldots$ & $\ldots$ & -0.58 \\
\hline J0241.1-0236 & 024103.87 & 3.9 & -023646.05 & 2.6 & $S$ & $0.42 \pm 0.06$ & $<32.6$ & $<26.3$ & $78.6 \pm 50.9$ & -0.95 \\
\hline J0241.1-0815 & 024105.48 & 4.2 & -081517.21 & 3.2 & $S$ & $0.91 \pm 0.13$ & $<48.7$ & $\ldots$ & $\ldots$ & 0.01 \\
\hline J0241.3-0533 & 024115.98 & 4.6 & -053348.79 & 4.3 & $S$ & $0.27 \pm 0.06$ & $<49.1$ & $<35.2$ & $168.4 \pm 43.3$ & -0.51 \\
\hline J0241.5-0235 & 024129.24 & 4.3 & -023512.31 & 4.2 & $S$ & $0.19 \pm 0.05$ & $<45.6$ & $<23.1$ & $166.7 \pm 25.7$ & -0.62 \\
\hline J0241.5-0431 & 024129.82 & 5.6 & -043122.68 & 4.2 & $S$ & $0.28 \pm 0.07$ & $<58.5$ & $<42.1$ & $71.4 \pm 40.9$ & -0.78 \\
\hline J0241.6-0418 & 024136.20 & 3.4 & -041820.45 & 2.1 & $S$ & $1.42 \pm 0.10$ & $<27.7$ & $<24.4$ & $73.9 \pm 37.1$ & -0.51 \\
\hline J0241.6-0647 & 024138.22 & 3.4 & -064728.03 & 2.0 & $S$ & $6.36 \pm 0.33$ & $25.2 \pm 6.2$ & $<21.8$ & $125.5 \pm 4.0$ & -0.71 \\
\hline J0241.6-0348 & 024138.38 & 3.5 & -034841.40 & 2.3 & $S$ & $0.63 \pm 0.06$ & $<26.6$ & $<16.8$ & $153.6 \pm 21.0$ & -0.78 \\
\hline J0241.7-0357 & 024140.41 & 5.0 & -035724.10 & 3.7 & $S$ & $0.22 \pm 0.05$ & $<48.8$ & $<30.0$ & $58.8 \pm 31.4$ & -0.67 \\
\hline \multirow[t]{2}{*}{ J0241.7-0349 } & 024142.42 & 4.4 & -034940.37 & 3.0 & $M$ & $0.41 \pm 0.07$ & $<55.7$ & $<21.7$ & $57.9 \pm 11.3$ & -1.01 \\
\hline & 024142.15 & 5.4 & -034928.61 & 4.6 & $C$ & $0.33 \pm 0.07$ & $<78.5$ & $<24.9$ & $134.4 \pm 10.4$ & \\
\hline J0241.7-0416 & 024144.25 & 4.6 & -041645.42 & 3.2 & $S$ & $0.51 \pm 0.09$ & $<56.1$ & $<32.9$ & $64.5 \pm 18.1$ & -0.60 \\
\hline J0241.9-0241 & 024152.10 & 4.0 & -024151.54 & 2.6 & $S$ & $0.44 \pm 0.06$ & $<40.0$ & $<24.0$ & $90.4 \pm 20.5$ & -0.47 \\
\hline J0241.9-0607 & 024156.30 & 4.1 & -060712.05 & 3.4 & $S$ & $0.28 \pm 0.05$ & $<38.1$ & $<21.5$ & $154.8 \pm 27.9$ & -0.45 \\
\hline J0242.1-0128 & 024208.16 & 3.5 & -012836.84 & 2.3 & $S$ & $1.49 \pm 0.12$ & $20.9 \pm 15.8$ & $<29.1$ & $150.9 \pm 21.1$ & -0.71 \\
\hline J0242.3-0548 & $0242 \quad 15.42$ & 4.4 & -054828.55 & 3.6 & $S$ & $0.57 \pm 0.10$ & $<52.8$ & $<41.5$ & $39.0 \pm 38.6$ & -0.60 \\
\hline J0242.3-0240 & 024218.52 & 3.5 & -024041.37 & 2.2 & $S$ & $0.74 \pm 0.07$ & $<23.9$ & $<20.7$ & $23.5 \pm 61.6$ & -0.67 \\
\hline
\end{tabular}


Table A.2. continued.

\begin{tabular}{|c|c|c|c|c|c|c|c|c|c|c|}
\hline Name & $\begin{array}{c}\text { RA } \\
\text { J2000 }\end{array}$ & $\begin{array}{c}\sigma_{\alpha} \\
\operatorname{arcsec}\end{array}$ & $\begin{array}{c}\text { Dec } \\
\text { J2000 }\end{array}$ & $\begin{array}{c}\sigma_{\delta} \\
\operatorname{arcsec}\end{array}$ & Type & $\begin{array}{c}S_{\text {comp }} \\
\mathrm{Jy}\end{array}$ & $\begin{array}{c}\text { Major axis } \\
\text { arcsec }\end{array}$ & $\begin{array}{c}\text { Minor axis } \\
\text { arcsec }\end{array}$ & $\begin{array}{c}\text { Position } \\
\text { Angle } \\
\end{array}$ & $\begin{array}{c}\text { Spectral } \\
\text { index } \alpha_{74}^{1400} \\
\end{array}$ \\
\hline J0242.3-0337 & 024218.79 & 5.0 & -033732.25 & 4.5 & $\bar{S}$ & $0.36 \pm 0.08$ & $<61.3$ & $<39.6$ & $35.8 \pm 27.7$ & -0.77 \\
\hline $\mathrm{J} 0242.3+0009$ & 024219.73 & 4.6 & +000908.49 & 4.7 & $S$ & $0.59 \pm 0.14$ & $<65.8$ & $<15.3$ & $31.2 \pm 10.9$ & -1.21 \\
\hline J0242.4-0012 & 024222.66 & 4.3 & -001239.94 & 5.6 & $S$ & $0.56 \pm 0.14$ & $<74.0$ & $<15.5$ & $17.2 \pm 9.9$ & -1.66 \\
\hline J0242.4-0408 & 024223.06 & 3.6 & -040858.40 & 2.3 & $S$ & $0.69 \pm 0.07$ & $<29.0$ & $<22.9$ & $113.6 \pm 36.6$ & -0.69 \\
\hline J0242.7-0000 & 024240.45 & 3.4 & -000045.91 & 2.0 & $S$ & $26.90 \pm 1.36$ & $23.1 \pm 5.4$ & $15.9 \pm 7.7$ & $46.5 \pm 3.8$ & -0.58 \\
\hline J0242.7-0311 & 024243.07 & 3.6 & -031145.87 & 2.2 & $S$ & $1.34 \pm 0.11$ & $33.3 \pm 12.1$ & $<24.4$ & $122.1 \pm 7.6$ & -0.48 \\
\hline J0242.7-0332 & 024243.44 & 3.8 & -033232.69 & 2.6 & $S$ & $0.67 \pm 0.08$ & $<35.6$ & $<31.5$ & $26.5 \pm 66.9$ & -0.71 \\
\hline J0242.8-0334 & 024246.69 & 4.3 & -033430.61 & 3.4 & $S$ & $0.74 \pm 0.11$ & $36.8 \pm 24.0$ & $<39.2$ & $139.1 \pm 17.5$ & -0.48 \\
\hline J0242.8-0157 & 024247.61 & 3.4 & -015747.39 & 2.1 & $S$ & $1.73 \pm 0.12$ & $<25.2$ & $<23.0$ & $6.3 \pm 51.0$ & -0.70 \\
\hline J0243.2-0550 & 024312.71 & 4.4 & -055054.67 & 3.4 & $S$ & $0.39 \pm 0.08$ & $<42.5$ & $<36.4$ & $122.2 \pm 76.1$ & 0.13 \\
\hline J0243.3-0356 & 024317.15 & 3.5 & -035658.22 & 2.2 & $S$ & $0.95 \pm 0.08$ & $<31.2$ & $<24.8$ & $118.9 \pm 26.7$ & -0.59 \\
\hline J0243.4-0433 & 024323.20 & 3.8 & -043336.48 & 2.5 & $S$ & $0.52 \pm 0.07$ & $<36.0$ & $<23.6$ & $76.5 \pm 23.1$ & -0.68 \\
\hline J0243.5-0232 & 024331.35 & 3.4 & -023255.83 & 2.1 & $S$ & $1.58 \pm 0.11$ & $<27.4$ & $<24.2$ & $120.9 \pm 38.8$ & -0.48 \\
\hline J0243.7-0718 & 024342.03 & 4.2 & -071803.45 & 3.2 & $S$ & $0.87 \pm 0.13$ & $35.5 \pm 23.6$ & $<34.0$ & $134.1 \pm 14.1$ & -0.80 \\
\hline J0243.8-0118 & 024346.06 & 4.8 & -011806.51 & 4.7 & $S$ & $0.53 \pm 0.12$ & $<56.7$ & $<42.5$ & $0.7 \pm 43.4$ & -1.20 \\
\hline J0243.8-0102 & 024348.96 & 3.9 & -010252.18 & 2.7 & $S$ & $0.95 \pm 0.12$ & $<36.8$ & $\ldots$ & $\ldots$ & -0.56 \\
\hline J0243.9-0305 & 024353.20 & 3.5 & -030506.67 & 2.2 & $S$ & $0.97 \pm 0.08$ & $<26.1$ & $<22.4$ & $136.6 \pm 46.9$ & -0.56 \\
\hline J0243.9-0027 & 024356.55 & 3.8 & -002728.85 & 2.6 & $S$ & $1.39 \pm 0.16$ & $23.5 \pm 22.8$ & $<29.5$ & $50.9 \pm 15.5$ & -0.86 \\
\hline J0244.0-0349 & 024400.89 & 3.9 & -034955.45 & 3.1 & $S$ & $0.45 \pm 0.07$ & $<47.0$ & $<24.2$ & $149.0 \pm 15.4$ & -0.75 \\
\hline J0244.2-0443 & 024411.35 & 4.7 & -044320.80 & 3.2 & $S$ & $0.32 \pm 0.06$ & $<45.6$ & $<28.6$ & $83.3 \pm 28.1$ & -0.59 \\
\hline J0244.3-0427 & 024420.77 & 3.5 & -042745.32 & 2.1 & $S$ & $1.25 \pm 0.10$ & $<33.2$ & $<23.3$ & $87.0 \pm 14.4$ & -0.58 \\
\hline J0244.4-0653 & 024423.17 & 3.5 & -065327.29 & 2.1 & $S$ & $2.26 \pm 0.15$ & $30.7 \pm 9.3$ & $<20.7$ & $118.5 \pm 5.6$ & -0.87 \\
\hline J0244.4-0527 & 024426.62 & 3.4 & -052715.94 & 2.0 & $S$ & $2.44 \pm 0.15$ & $21.6 \pm 9.7$ & $<22.0$ & $105.4 \pm 8.8$ & -0.78 \\
\hline J0244.6-0310 & 024433.79 & 5.2 & -031023.51 & 3.8 & $S$ & $0.25 \pm 0.06$ & $<48.5$ & $<30.7$ & $112.6 \pm 35.3$ & -0.77 \\
\hline J0244.6-0648 & 024435.98 & 4.7 & -064849.21 & 3.9 & $S$ & $0.35 \pm 0.08$ & $<52.2$ & $<25.3$ & $136.8 \pm 20.1$ & -1.35 \\
\hline \multirow[t]{2}{*}{ J0244.8-0547 } & 024450.06 & 7.9 & -054752.00 & 3.7 & $M$ & $0.92 \pm 0.18$ & $82.9 \pm 38.1$ & $<44.9$ & $91.6 \pm 8.0$ & -0.71 \\
\hline & 024450.52 & 4.9 & -054725.72 & 3.4 & $C$ & $0.47 \pm 0.09$ & $<60.4$ & $<28.3$ & $119.2 \pm 14.8$ & \\
\hline J0244.9-0548 & 024454.01 & 4.7 & -054828.31 & 3.2 & $S$ & $1.15 \pm 0.16$ & $54.5 \pm 21.8$ & $<43.7$ & $115.4 \pm 11.5$ & -0.64 \\
\hline J0244.9-0257 & 024456.51 & 6.6 & -025746.80 & 3.9 & $S$ & $0.47 \pm 0.10$ & $<85.3$ & $<36.3$ & $107.7 \pm 12.6$ & -0.87 \\
\hline J0245.0-0447 & 024457.66 & 3.7 & -044748.26 & 2.5 & $S$ & $0.62 \pm 0.07$ & $<31.7$ & $<28.6$ & $136.8 \pm 85.8$ & -0.94 \\
\hline J0245.1-0504 & 024508.04 & 5.0 & -050443.14 & 4.3 & $S$ & $0.44 \pm 0.09$ & $<65.1$ & $<34.4$ & $136.9 \pm 18.0$ & -0.97 \\
\hline J0245.1-0452 & 024508.38 & 3.7 & -045225.54 & 2.6 & $S$ & $0.53 \pm 0.07$ & $<30.7$ & $<26.1$ & $135.0 \pm 61.0$ & -0.82 \\
\hline J0245.2-0133 & 024509.73 & 3.5 & -013350.27 & 2.2 & $S$ & $1.59 \pm 0.13$ & $<34.7$ & $<27.9$ & $79.4 \pm 23.7$ & -0.89 \\
\hline J0245.3-0256 & 024519.65 & 4.4 & -025627.30 & 4.3 & $S$ & $0.35 \pm 0.08$ & $<53.2$ & $<30.8$ & $160.5 \pm 24.5$ & -0.52 \\
\hline J0245.6-0646 & 024538.53 & 4.4 & -064616.39 & 3.1 & $S$ & $0.55 \pm 0.10$ & $<51.1$ & $<24.8$ & $121.9 \pm 15.9$ & -0.73 \\
\hline \multirow[t]{2}{*}{$\mathrm{J} 0245.8-0300$} & 024546.12 & 4.0 & -030059.87 & 2.7 & $M$ & $0.37 \pm 0.06$ & $<31.9$ & $<22.4$ & $100.8 \pm 37.5$ & -0.65 \\
\hline & 024546.36 & 4.7 & -030044.97 & 3.0 & $C$ & $0.26 \pm 0.05$ & $<46.3$ & $<17.3$ & $69.5 \pm 16.8$ & \\
\hline J0245.8-0518 & 024550.48 & 3.8 & -051857.56 & 2.6 & $S$ & $0.62 \pm 0.08$ & $<40.7$ & $<21.0$ & $126.0 \pm 14.3$ & -0.70 \\
\hline J0246.1-0600 & 024603.51 & 5.2 & -060025.01 & 3.6 & $S$ & $0.38 \pm 0.08$ & $<57.8$ & $<29.0$ & $115.2 \pm 18.9$ & -0.71 \\
\hline J0246.2-0335 & 024611.84 & 3.9 & -033523.95 & 2.8 & $S$ & $0.48 \pm 0.07$ & $<33.7$ & $<29.6$ & $102.0 \pm 82.4$ & -0.68 \\
\hline J0246.2-0228 & 024612.46 & 3.7 & -022835.71 & 2.7 & $S$ & $0.69 \pm 0.08$ & $<36.9$ & $<25.2$ & $164.9 \pm 23.2$ & -0.89 \\
\hline J0246.3-0131 & 024618.64 & 3.8 & -013136.07 & 2.7 & $S$ & $0.81 \pm 0.10$ & $<37.9$ & $<29.5$ & $129.7 \pm 34.5$ & -0.59 \\
\hline J0246.4-0508 & 024622.39 & 5.2 & -050857.41 & 4.3 & $S$ & $0.58 \pm 0.12$ & $<66.2$ & $<43.8$ & $47.8 \pm 25.6$ & -0.89 \\
\hline J0246.4-0449 & 024622.93 & 3.4 & -044929.50 & 2.1 & $S$ & $2.86 \pm 0.18$ & $41.2 \pm 7.8$ & $19.5 \pm 11.6$ & $23.7 \pm 5.5$ & -0.79 \\
\hline J0246.8-0145 & 024647.11 & 3.6 & -014533.12 & 2.3 & $S$ & $1.36 \pm 0.12$ & $<35.2$ & $<25.8$ & $113.0 \pm 19.7$ & -0.78 \\
\hline J0247.1-0529 & 024707.04 & 4.0 & -052958.15 & 3.0 & $S$ & $0.47 \pm 0.08$ & $<40.4$ & $<20.0$ & $143.4 \pm 18.5$ & -0.69 \\
\hline J0247.1-0528 & 024707.48 & 4.5 & -052812.31 & 3.6 & $S$ & $0.35 \pm 0.08$ & $<46.8$ & $<24.9$ & $133.0 \pm 23.4$ & -0.75 \\
\hline J0247.2-0354 & 024709.65 & 3.9 & -035445.17 & 2.8 & $S$ & $0.74 \pm 0.10$ & $<38.4$ & $\ldots$ & $\ldots$ & -0.76 \\
\hline J0247.3-0554 & 024716.56 & 3.5 & -055441.18 & 2.2 & $S$ & $2.05 \pm 0.15$ & $28.1 \pm 11.0$ & $<25.6$ & $138.5 \pm 9.5$ & -0.64 \\
\hline J0247.4-0338 & 024725.04 & 4.4 & -033804.59 & 2.8 & $S$ & $0.31 \pm 0.06$ & $<40.2$ & $<20.0$ & $93.7 \pm 22.2$ & -0.43 \\
\hline J0247.5-0458 & 024731.42 & 5.7 & -045814.87 & 4.3 & $S$ & $0.62 \pm 0.13$ & $<66.4$ & $<52.2$ & $86.1 \pm 44.3$ & -0.84 \\
\hline J0247.6-0538 & 024735.07 & 4.8 & -053840.37 & 4.5 & $S$ & $0.47 \pm 0.10$ & $<62.2$ & $<34.2$ & $148.2 \pm 20.1$ & -0.85 \\
\hline J0248.1-0331 & 024803.89 & 4.8 & -033141.64 & 3.4 & $S$ & $0.68 \pm 0.12$ & $<55.6$ & $<40.6$ & $96.8 \pm 30.3$ & -0.95 \\
\hline J0248.1-0432 & 024805.34 & 6.0 & -043242.97 & 4.3 & $S$ & $0.56 \pm 0.13$ & $<68.2$ & $<50.3$ & $85.1 \pm 37.7$ & -0.65 \\
\hline J0248.2-0415 & 024809.86 & 4.3 & -041502.18 & 3.2 & $S$ & $0.79 \pm 0.12$ & $<48.7$ & $<43.4$ & $111.4 \pm 73.9$ & -0.36 \\
\hline J0248.2-0415 & 024812.41 & 4.2 & -041554.16 & 4.1 & $S$ & $1.45 \pm 0.19$ & $61.5 \pm 21.7$ & $<46.9$ & $159.1 \pm 10.3$ & -0.60 \\
\hline \multirow[t]{2}{*}{ J0248.2-0313 } & 024813.95 & 4.1 & -031316.64 & 3.0 & $M$ & $0.48 \pm 0.08$ & $<46.1$ & $<15.5$ & $133.4 \pm 12.3$ & -0.75 \\
\hline & 024814.89 & 4.6 & -03 1309.12 & 4.0 & $C$ & $0.30 \pm 0.07$ & $<53.1$ & $<17.5$ & $38.9 \pm 15.6$ & \\
\hline J0248.5-0512 & 024832.47 & 4.2 & -051259.20 & 3.2 & $S$ & $0.38 \pm 0.07$ & $<37.7$ & $<23.8$ & $138.3 \pm 32.7$ & -0.62 \\
\hline
\end{tabular}

An asterisk (*) after the sourcename indicates this source was also detected at $325 \mathrm{MHz}$.

1. Source Name.

2. Right Ascension (J2000)

3. Right Ascension uncertainty in arcseconds.

4. Declination (J2000)

5. Declination uncertainty in arcseconds.

6. Source type: Single (S), Multiple (M) or Component (C) of multiple source.

7. Flux density of individual componant in Jy as derived through the Gaussian fitting method.

8. Size of Major Axis in arcseconds, or the $2 \sigma$ upper limit if the source is unresolved.

9. Size of Minor Axis in arcseconds, or the $2 \sigma$ upper limit if the source is unresolved.

10. PA of Major axis in degrees.

11. Spectral index with respect to NVSS flux density $(1.4 \mathrm{GHz})$. 